\title{
Examining Extra Neutral Gauge Bosons in Non-Universal Models and Exploring the Phenomenology of the Bestest Little Higgs Model at the LHC
}

by

\author{
Travis Anthony William Martin
}

A thesis submitted to the Faculty of Graduate and Postdoctoral Affairs in partial fulfillment of the requirements for the degree of

Doctor of Philosophy

in

Physics

Ottawa-Carleton Institute for Physics

Department of Physics

Carleton University

Ottawa, Ontario, Canada

August 31, 2012

Copyright (C)

2012 - Travis Anthony William Martin 
Library and Archives

Canada

Published Heritage

Branch

395 Wellington Street

Ottawa ON K1A ON4

Canada
Bibliothèque et

Archives Canada

Direction du

Patrimoine de l'édition

395 , rue Wellington

Ottawa ON K1A ON4

Canada
Your file Votre référence

ISBN: 978-0-494-93684-9

Our file Notre référence

ISBN: $978-0-494-93684-9$
NOTICE:

The author has granted a nonexclusive license allowing Library and Archives Canada to reproduce, publish, archive, preserve, conserve, communicate to the public by telecommunication or on the Internet, loan, distrbute and sell theses worldwide, for commercial or noncommercial purposes, in microform, paper, electronic and/or any other formats.

The author retains copyright ownership and moral rights in this thesis. Neither the thesis nor substantial extracts from it may be printed or otherwise reproduced without the author's permission.
AVIS:

L'auteur a accordé une licence non exclusive permettant à la Bibliothèque et Archives Canada de reproduire, publier, archiver, sauvegarder, conserver, transmettre au public par télécommunication ou par l'Internet, prêter, distribuer et vendre des thèses partout dans le monde, à des fins commerciales ou autres, sur support microforme, papier, électronique et/ou autres formats.

L'auteur conserve la propriété du droit d'auteur et des droits moraux qui protege cette thèse. $\mathrm{Ni}$ la thèse ni des extraits substantiels de celle-ci ne doivent être imprimés ou autrement reproduits sans son autorisation.
In compliance with the Canadian Privacy Act some supporting forms may have been removed from this thesis.

While these forms may be included in the document page count, their removal does not represent any loss of content from the thesis.
Conformément à la loi canadienne sur la protection de la vie privée, quelques formulaires secondaires ont été enlevés de cette thèse.

Bien que ces formulaires aient inclus dans la pagination, il n'y aura aucun contenu manquant. 


\section{Abstract}

This thesis expands upon the literature in two distinct areas - exploring properties of extra neutral gauge boson $\left(Z^{\prime}\right)$ observables, and exploring the phenomenology of the Bestest Little Higgs model at the Large Hadron Collider.

In the first Part, it is shown that two well known $Z^{\prime}$ observables, the centre-edge asymmetry and forward-backward asymmetry, can be calculated using only the pseudorapidity values of the final state decay products. The benefit of this method is that pseudorapidity can be measured very precisely, which reduces systematic uncertainties that arise from compounding errors in calculating the full kinematics of each event. The forward-backward asymmetry of hadronic final states with large $\Delta R$ is also simplified, as the pseudorapidity of the centroid of such fat jets can be used. Additionally, this thesis explores the application of cross section ratios $\left(Z^{\prime} \rightarrow t \bar{t}\right.$, $Z^{\prime} \rightarrow b \bar{b}$, and $Z^{\prime} \rightarrow \tau^{+} \tau^{-}$to $Z^{\prime} \rightarrow \mu^{+} \mu^{-}$) to exploring the class of models with non-universal couplings, such as Topcolor and Technicolor models. It is clear that uniquely identifying non-universal couplings necessarily requires measuring decays of the $Z^{\prime}$ to third generation final states.

In the second Part, the heavy quark sector of the Bestest Little Higgs model is explored. With an integrated luminosity of $1.14 \mathrm{fb}^{-1}$ at the LHC $(7 \mathrm{TeV})$, the discovery reach for new heavy top quarks in the model is found to be above $350 \mathrm{GeV}$ for two sample regions of parameter space. This is lower than most experimental studies suggest since the lightest heavy top partners in the Bestest Little Higgs model 
decay to a combination of $b W, t Z$ and $t h$ final states, rather than to a singular decay mode. Expanding on this, measurements of the masses of the heavy top partners, heavy bottom quark and exotic $+5 / 3$ charge quark are simulated in the $b \bar{b} W^{+} W^{-}$and $t \bar{t} W^{+} W^{-}$final states to explore the capability of the LHC experiments to determine the Yukawa coupling parameters, which are necessary for understanding the degree of cancellation for the top loop contribution to the Higgs mass. With an integrated luminosity of $100 \mathrm{fb}^{-1}$ for $14 \mathrm{TeV}$ collisions, it should be possible to measure the masses of either the $T_{6}$ and $T_{5}$ quarks or the $T_{6}$ and the $B$ quarks, the combinations of which give insight to different Yukawa parameters, respectively. Measuring all heavy quark states will prove to be difficult without a significant increase in luminosity over the design goal of $100 \mathrm{fb}^{-1}$. The heaviest top partner states are suppressed by the available phase space and decay primarily to non-Standard Model final states, which results in an event rate that is too low to be seen at $100 \mathrm{fb}^{-1}$ of integrated luminosity. 
To my family and friends, for all of their support. 


\section{Acknowledgments}

This research was supported in part by the Natural Sciences and Engineering Research Council of Canada and the Ontario Graduate Scholarship program.

Many people have provided assistance and guidance in the development of the research presented here. In addition to my supervisors, Stephen Godfrey and Thomas Grégoire, who have been incredibly supportive and helpful, I would like to acknowledge the assistance of the following people:

With regards to the pseudorapidity techniques, I would like to thank Malachi Schram and Isabel Trigger for helpful discussions.

With regards to the use of third generation final states to explore non-universal models, I would like to thank Dag Gillberg, David Morrissey, Dugan O'Neil, Oliver Stelzer-Chilton and Isabel Trigger for helpful discussions and explanations.

With regards to the Bestest Little Higgs model phenomenology, I would like to thank Pat Kalyniak for guidance and assistance as one of my collaborators. Additionally, I would like to thank Edmond Berger, Qing-Hong Cao, Chris Hill, Yanjun $\mathrm{Tu}$, Martin Schmaltz, and Jesse Thaler for helpful discussions and elaborations on the ideas developed in their publications.

Lastly, I would like to thank Heather Logan and Bruce Campbell, who have both given immense time to elaborate on the finer details of many particle physics concepts. 


\section{Preface}

\section{Statement of Originality}

I, Travis A. W. Martin, declare that this thesis is comprised of original work performed by me, under the guidance of my supervisors, Stephen Godfrey and Thomas Grégoire, as summarized below. This thesis comprises a summary of my research performed at Carleton University between 2007 and 2012.

The ideas relating to the use of pseudorapidity to simplify and improve calculations at the LHC, included within Part I, were published in Physical Review D [1]. All ideas and calculations were my own, and I acted as the primary author, with one caveat: the idea for the use of pseudorapidity to measure the Centre-Edge Asymmetry was introduced by my supervisor, Stephen Godfrey.

The use of cross section ratios to distinguish models with non-universal couplings within Part I is my original work, and follows from the work published in my Master's Thesis in 2007. While these observables were first examined in my Master's Thesis, the application of them to uniquely identify non-universal couplings is new and was published in Physical Review D [2]. For this analysis, I acted as the primary author and performed all calculations.

The Bestest Little Higgs model, detailed in Part II, was first created by Martin Schmaltz, Daniel Stolarski, and Jesse Thaler [3]. However, this initial publication did not describe the phenomenology of the model in detail. The details of the mass 
eigenstates and particle mixing and interactions, discussed in Chapter 6 and Appendices $\mathrm{B}$ and $\mathrm{C}$, were established by a collaboration involving myself, Ken Moats, Stephen Godfrey, Pat Kalyniak and Thomas Grégoire.

These details were used to establish the methods for determining the discovery reach and Yukawa coupling information, which were my own ideas and my own calculations. The discovery reach for heavy quarks in the Bestest Little Higgs model was first explored in a publication in Journal of High Energy Physics [4], for which Ken Moats and I acted as the primary authors. The ideas and calculations involved in the determination of the Yukawa couplings and model parameters is unpublished at the time of submitting this thesis. 


\section{Table of Contents}

Abstract $\quad$ i

Acknowledgments $\quad$ iv

Preface $\quad$ v

Table of Contents vii

List of Tables $\quad$ xi

List of Figures $\quad$ xii

1 Introduction 1

2 The Standard Model of Particle Physics 5

2.1 Overview of the Standard Model . . . . . . . . . . . . . . 5

2.1.1 Electroweak Gauge Bosons and their Interactions . . . . . . 5

2.1.2 Electroweak Symmetry Breaking . . . . . . . . . . . . 7

2.1.3 Strong Interactions . . . . . . . . . . . . . . 9

2.1 .4 Summary .......................... 10

2.2 Physics Beyond the Standard Model . . . . . . . . . . . . . . . . 12

2.2.1 Indication for Physics at the TeV Scale . . . . . . . . . . 12

2.2.2 The Large Hadron Collider . . . . . . . . . . . . . . . . 14 


\section{Examining Non-Universal Extra Neutral Gauge Bosons}

at the LHC

3 Extra Neutral Gauge Bosons $\quad 17$

3.1 Motivation . . . . . . . . . . . . . . . . 17

3.2 Models . . . . . . . . . . . . . . . . . . . . . 21

3.2 .1 Ununified Model (UUM) $\ldots \ldots \ldots \ldots$

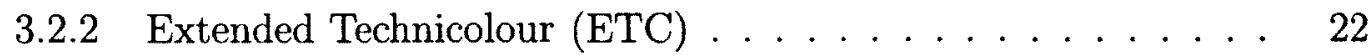

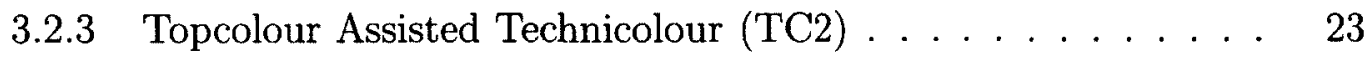

4 Calculations $\quad 24$

4.1 Neutral Gauge Bosons . . . . . . . . . . . . . . . . . . 24

4.2 Observables . . . . . . . . . . . . . . . 27

4.2 .1 Centre-Edge Asymmetry . . . . . . . . . . . . . . . . 29

4.2 .2 Forward-Backward Asymmetry . . . . . . . . . . . 32

4.2 .3 Third Generation Ratios . . . . . . . . . . . . . . . 35

4.3 Identification and Backgrounds $\ldots \ldots \ldots \ldots \ldots \ldots$

4.3.1 Muons and Electrons . . . . . . . . . . . . . . 38

4.3 .2 Bottom Quarks . . . . . . . . . . . . . . . . . 38

4.3 .3 Top Quarks . . . . . . . . . . . . . . . . . . . . . . . 39

4.3 .4 Tau Leptons . . . . . . . . . . . . . . . . . . . . 40

4.3 .5 Tagging Summary $\ldots \ldots \ldots \ldots$. . . . . . . . 41

4.4 Kinematic Cuts . . . . . . . . . . . . . . . . . 41

5 Results $\quad 46$

5.1 Centre-Edge Asymmetry . . . . . . . . . . . . 46

5.2 Forward-Backward Asymmetry $\ldots \ldots \ldots \ldots \ldots$

5.3 Third Generation Ratios . . . . . . . . . . . . . . . 55 
5.3.1 Model Discrimination using $t \bar{t}$ and $b \bar{b}$ to $\mu^{+} \mu^{-}$Production Ratios 55

5.3.2 Extracting Mixing Parameters Using $R_{\tau / \mu} \ldots \ldots \ldots 6$

\section{Exploring the Phenomenology of the Bestest Little Higgs Model at the LHC}

6 Model Details $\quad 66$

6.1 Mass Eigenstates and Particle Masses . . . . . . . . . . . . 67

6.1.1 Scalar Sector, Higgs Potential and Spontaneous Symmetry

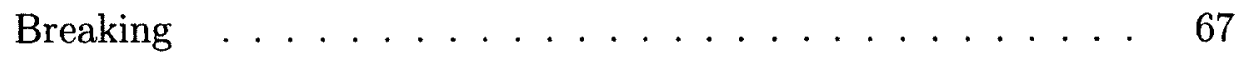

6.1 .2 Gauge Bosons . . . . . . . . . . . . . . . . . . 72

6.1 .3 Heavy Fermions . . . . . . . . . . . . . . . 74

6.1 .4 Light Fermions . . . . . . . . . . . . . . . 80

6.2 Interactions in the Bestest Little Higgs Model . . . . . . . . . . 81

$\begin{array}{lll}7 & \text { Calculations } & 85\end{array}$

7.1 Heavy Quark Discovery Limits . . . . . . . . . . . . . . . 86

7.2 Measuring Masses and Determining Yukawa Couplings . . . . . . 92

7.2.1 Heavy Top-Partners . . . . . . . . . . . . . . 95

7.2.2 Heavy Bottom Partner and Exotic Quark . . . . . . . 98

7.2 .3 Tagging Efficiency $\ldots \ldots \ldots \ldots . \ldots \ldots$

8 Results 101

8.1 Discovery Limits . . . . . . . . . . . . . . . . . . . 101

8.2 Determining Yukawa Couplings . . . . . . . . . . 106

8.2 .1 Heavy Top Partners . . . . . . . . . . . . . 106

8.2 .2 Exotic and Bottom Partner . . . . . . . . . . 114

8.2.3 Constraining Yukawa Mixing Angles . . . . . . . . 116 
9 Summary and Conclusion

9.1 Using Pseudorapidity to Improve $Z^{\prime}$ measurements . . . . . . . . . . 124

9.2 Distinguishing a $Z^{\prime}$ with Non-Universal Couplings . . . . . . . . . . . 126

9.3 Determining Yukawa Couplings in the Bestest Little Higgs Model . 127

References

Appendix A Neutral Resonance Model Details

142

A.1 R-Parity Violating $\tilde{\nu}($ spin- 0$) \ldots \ldots \ldots 142$

A.2 Randall-Sundrum Graviton (spin-2) . . . . . . . . . . 143

A.3 Other $Z^{\prime}$ Models . . . . . . . . . . . . . . . . 143

A.3.1 E6 Models (E6) . . . . . . . . . . . . . . 143

A.3.2 Left-Right Symmetric Models (LR \& ALR) . . . . . . . . . 145

A.3.3 Little Higgs Models . . . . . . . . . . . . . . . . . . . . 146

A.3.4 3-3-1 Models (331) . . . . . . . . . . . . . 147

Appendix B Two Higgs Doublet Model Relations 149

B.1 Coleman-Weinberg Potential . . . . . . . . . . . . 150

B.2 Relation to $2 \mathrm{HDM} \ldots \ldots \ldots \ldots \ldots \ldots$

Appendix C Mass Eigenstates in the Bestest Little Higgs Model 156

C.1 Scalar Sector . . . . . . . . . . . . . . 156

C.2 Gauge Sector . . . . . . . . . . . . . . . 158

C.3 Fermion Sector . . . . . . . . . . . . . . 160

Appendix D Generators of $S O(6)$ and $S U(2) \quad 164$ 


\section{List of Tables}

2.1 Standard Model Particles and their Properties . . . . . . . . . . 11

2.2 Particle quantum numbers in the SM. . . . . . . . . . . . 12

4.1 Summary of ATLAS tagging efficiencies and fake rates. . . . . . . 41

$5.1 \quad$ Table of $\tilde{A}_{C E}$ values. $\ldots \ldots \ldots \ldots \ldots$

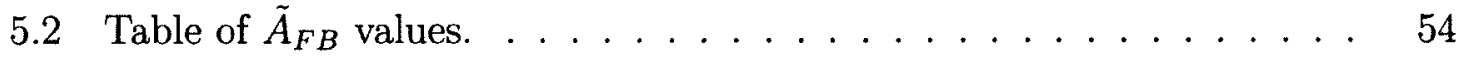

7.1 Heavy quark pair production branching ratios to the $b W$ final state. $\quad 90$

7.2 Heavy quark pair production branching ratios to the $t Z$ final state. . 91

8.1 Heavy quark pair production cross section for the $b \bar{b} W^{+} W^{-}$decay mode. . . . . . . . . . . . . . . . . . . . . 103

8.2 Heavy quark pair production cross section for the $t \bar{t} Z Z$ decay mode. 104

8.3 Masses and relevant branching ratios for all six heavy quarks in the BLH model. . . . . . . . . . . . . . . . . 107

A.1 Couplings of SM fermions to the $Z^{\prime}$ in the $E_{6} \psi$ and $\chi$ Models $\ldots .144$

A.2 Couplings of SM fermions to the $Z^{\prime}$ in the Simplest Little Higgs model 147

A.3 Couplings of SM fermions to the $Z^{\prime}$ in the Anomaly Free Simple Little Higgs model . . . . . . . . . . . . . . . . . . . . . . . 148

A.4 Couplings of the SM Fermions in the 3-3-1 Model model . . . . . . 148 


\section{List of Figures}

2.1 Electroweak vertices describing the interactions of gauge bosons and

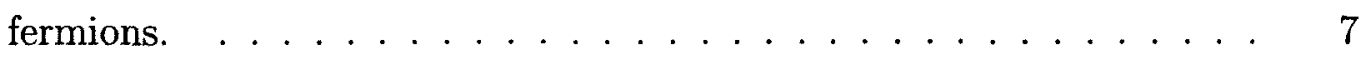

2.2 Three and four point vertices involving only electroweak gauge bosons $-W^{ \pm}, \gamma$ and $Z^{0} . \ldots \ldots \ldots \ldots \ldots \ldots \ldots \ldots \ldots$

2.3 QCD vertices in the Standard Model . . . . . . . . . . 10

2.4 One loop divergent contributions to the Higgs mass. . . . . . . . 14

4.1 Feynman diagram representing the Drell-Yan production of leptons at a hadron collider. . . . . . . . . . . . . . 25

4.2 Symmetric and anti-symmetric pdf combinations versus $Z^{\prime}$ rapidity, showing the relative contributions to symmetric and anti-symmetric decays. . . . . . . . . . . . . . . . . . . .

4.3 Idealized angular distribution of spin-0, spin-1 and spin-2 s-channel

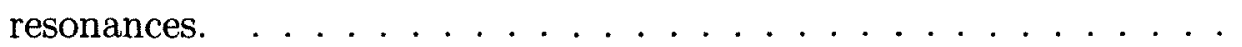

$4.4 b \bar{b}$ invariant mass distribution, including $Z^{\prime}$ production and QCD back-

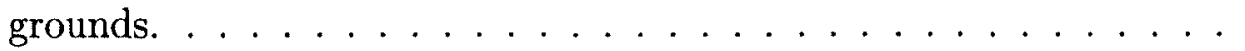

4.5 Fraction of events that remain within an invariant mass window of $\pm 2.5 \Gamma_{Z^{\prime}}$ about the $Z^{\prime}$ peak due to the effects of $3 \%, 5 \%, 7 \%$ and $9 \%$

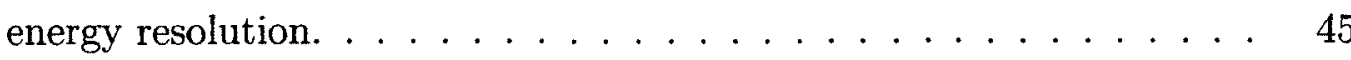

5.1 Normalized $\Delta \eta$ distribution including PDF and detector effects. $\ldots 47$ 
5.2 Rapidity distribution of difference between forward and backward tagged events. . . . . . . . . . . . . . . . . . 49

5.3 On-peak versus off-peak forward-backward asymmetry results for a variety of extra neutral gauge bosons. . . . . . . . . . . . 50

5.4 On-peak forward-backward asymmetry in the $b \bar{b}$ and $t \bar{t}$ final state, arising from a heavy $Z^{\prime} \ldots \ldots \ldots \ldots 2$

5.5 On-peak forward-backward asymmetry in the $b \vec{b}$ and $t \bar{t}$ final state, arising from a heavy $Z^{\prime} \ldots \ldots \ldots 53$

5.6 Distribution of $R_{t / \mu}$ versus $R_{b / \mu}$ values for models with universal couplings. .......................... 56

5.7 Distribution of $R_{t / \mu}$ versus $R_{b / \mu}$ values for models with non-universal couplings. . . . . . . . . . . . . . . . 57

5.8 Distribution of $R_{t / \mu}$ versus $R_{b / \mu}$ including smearing effects, for models with universal couplings. ................. . . 58

5.9 Distribution of $R_{t / \mu}$ versus $R_{b / \mu}$ including smearing effects, for models with non-universal couplings. . . . . . . . . . . . . . . 59

5.10 Evolution of $R_{b / \mu}$ and $R_{t / \mu}$ values due to varying free model parameters. 60

$5.11 R_{\tau / \mu}$ ratio for all $Z^{\prime}$ models examined. . . . . . . . . . . 62

$5.12 \chi^{2}$ analysis of mixing parameter for non-universal models from $R_{\tau / \mu}$ measurements. .................... . . 64

6.1 Constraints on $\theta_{12}$ and $\theta_{13}$ based on perturbativity and fine-tuning considerations. ......................... 78

6.2 Masses of the heavy quarks in the BLH model, as $\theta_{12}$ and $\theta_{13}$ are varied. 79

6.3 Mass difference between the $T$ state and the $B$ state, as $\theta_{12}$ and $\theta_{13}$ are varied. ........................ 80

7.1 Enlarged view of the heavy quark parameter space showing placement of isolated and non-isolated scenarios, for $f=700 \mathrm{GeV}$. 
7.2 Branching ratios for the three lightest heavy quarks for the isolated and non-isolated scenarios $\left(T_{6}, T_{5}\right.$ and $\left.T_{b}^{2 / 3}\right) \ldots \ldots \ldots$

8.1 Expected cross section times branching ratio in the $b W$ final state, and corresponding current experimental limits from CMS. . . . . . . . . 102

8.2 Expected cross section times branching ratio in the $t Z$ final state, and corresponding current experimental limits from CMS. . . . . . . . 105

8.3 Invariant mass distribution of the $b W$ candidate (log scale) signal and backgrounds. . . . . . . . . . . . . . . . . . . 108

8.4 Invariant mass distribution of the $b W$ candidate (linear scale). . . . 109

8.5 Pseudo-Dalitz plot showing regions of phase space for the primary heavy top partner signal and background production modes. . . . . 110

8.6 Invariant mass distribution of the $b W$ candidate (linear scale) after $p_{T}$ cuts.

8.7 Background subtracted signal events for heavy top partners in scenario 1, fit with a Breit-Wigner distribution. . . . . . . . . . . 112

8.8 Background subtracted signal events for heavy top partners in scenario 2 , fit with a Breit-Wigner distribution. . . . . . . . . . . . . . 113

8.9 Invariant mass of the top quark candidate ( $\mathrm{jW}$ ) intermediate state for the $b \bar{b}+4 j$ background. . . . . . . . . . . . . . . . 115

8.10 Invariant mass distribution of the reconstructed $b W W$ system (log scale).116

8.11 Invariant mass distribution of the reconstructed $b W W$ system (linear

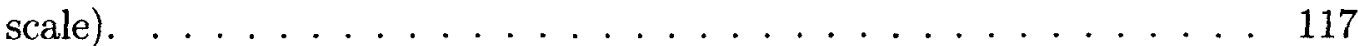

8.12 Background subtracted signal events for the heavy bottom partner and exotic quark, fit with two Breit-Wigner functions. . . . . . . . . . . . 118

$8.13 \chi^{2}$ analysis of mass results in scenario 1 , assuming purely statistical errors. . . . . . . . . . . . . . . . . . . . . 119 
$8.14 \chi^{2}$ analysis of mass results in scenario 2 , assuming purely statistical errors. . . . . . . . . . . . . . . . . 120

$8.15 \chi^{2}$ analysis of mass results in scenario 1 , using both statistical and $1.53 \%$ systematic error. . . . . . . . . . . . . . . . 122

$8.16 \chi^{2}$ analysis of mass results in scenario 2 , using both statistical and $1.53 \%$ systematic error. . . . . . . . . . . . . . . . . 123

9.1 Single production mechanism of the exotic $T_{b}^{5 / 3}$ quark. . . . . . 129 


\section{Chapter 1}

\section{Introduction}

The field of particle physics explores the fundamental interactions of subatomic particles, and examines the physics present in the universe only moments after the Big Bang. The Standard Model (SM) of particle physics, established in the 1970s, is currently the best theoretical description of these interactions and has had tremendous success in both predicting the existence of new particles and simulating the results of high energy particle collisions. However, the SM has not yet been completely verified, as one key component - the Higgs boson - has not yet been observed or measured, though there are now hints.

From 1983 to 2011, the Tevatron facility in Illinois, USA, pushed the high energy frontier by colliding protons and anti-protons at energies up to $1.96 \mathrm{TeV}$. In 2010 , the Large Hadron Collider (LHC) in Geneva, Switzerland, began proton-proton collisions at energies of $7 \mathrm{TeV}$, further expanding the high energy frontier of particle collision experiments. While the Tevatron facility successfully verified many aspects of the Standard Model and discovered the existence of the top quark, it was only able to put stringent limits on the existence of new physics that might exist beyond the SM. The goal of the LHC will be to completely verify the particle content of the SM (i.e. find the Higgs boson), if possible, and to search for physics beyond the Standard Model (BSM). 
Even though the SM has been very successful, there are several reasons to search for new physics at energies beyond the SM. The first evidence of this new physics should arise in the TeV energy range. These reasons include, but are not limited to, the following observations [5]:

- the Super Kamiokande neutrino experiment [6], and the SNO experiment [7], have observed neutrino oscillations, indicating that neutrinos have mass, in contradiction to the current SM implementation of massless neutrinos;

- astronomical observations of galaxy rotations and galaxy collisions, such as the Bullet Cluster [8], indicate that there is some form of very weakly interacting dark matter that dominates the mass content of the Universe but is not included in the SM;

- the universe appears to be composed of matter, as opposed to anti-matter, to a degree that is not explainable without some greater degree of Charge-Parity (CP) symmetry violation than exists in the SM;

- the SM contains three generations of fermions, but does not have a physical explanation for the existence of these generations [5];

- there exists a huge energy range between the weak scale $(\approx 250 \mathrm{GeV})$ and the energy scale that gravity becomes strong $\left(M_{\text {Planck }}=10^{19} \mathrm{GeV}\right)$, which is known as the hierarchy problem within the SM.

Additionally, the SM contains 26 arbitrary parameters, including coupling strengths, masses and mixing angles. A fundamental theory with physical explanations for the values of these parameters is more desirable.

Due to these reasons and others, particle theorists believe that there must be some physics beyond the SM. A number of different theories have arisen to address 
these issues, including, but not limited to, extra dimensions, grand unification, supersymmetry, strong dynamics and collective symmetry breaking. These new theories selectively resolve a subset of the known issues with the SM by introducing new interactions that should be visible at energies greater than the electroweak scale in the SM, but at energies that will be explored at the LHC.

Following a brief introduction to the SM in Chapter 2, this thesis is split into two parts: the first part explores physics beyond the standard model through the study of the phenomenology of extra neutral gauge bosons, while the second presents a study of signatures of a new Little Higgs type model, known as the Bestest Little Higgs model.

\section{Part I: Examining extra neutral gauge bosons with non-universal couplings}

Common to many models of new physics is the presence of new gauge particles and interactions, which are heavy partners of the weak gauge bosons of the SM. A new heavy, neutral gauge boson, commonly called a $Z^{\prime}$, has been studied in detail in terms of grand unified theories [9-12], but relatively little effort has been made to explore $Z^{\prime}$ signatures from models arising from other types of symmetries - specifically those that result in non-universal couplings [13-29]. Part I of this thesis explores a number of new techniques for improving asymmetry measurements as well as presents a study of observables that may be able to distinguish properties of a $Z^{\prime}$ with non-universal couplings. Chapter 3 discusses the details of extra neutral gauge bosons, and the models with non-universal couplings that are examined, while Chapter 4 reviews the details of the observables and the calculations. An estimation of the capabilities of the detectors at the LHC to perform these measurements is given in Chapter 5 . 


\section{Part II: Exploring the phenomenology of the Bestest Little Higgs Model}

In Part II of this thesis, the details of the phenomenology of a newly proposed Little Higgs model [30-32], entitled the Bestest Little Higgs model [3], are explored, focusing on the discovery potential and measurements in the heavy quark sector. This model addresses a number of issues arising in other Little Higgs models and includes unique phenomenology that is not present in other models. In Chapter 6, details of the Bestest Little Higgs model are discussed, including the Higgs potential and electroweak symmetry breaking, and the masses of all of the new particle states. Chapter 7 discusses the details of the discovery of heavy quarks through pair production, and the details of determining Yukawa parameters from the masses of the heavy quarks. Chapter 8 presents the results of the heavy quark phenomenology. 


\section{Chapter 2}

\section{The Standard Model of Particle Physics}

To reveal evidence of physics beyond the Standard Model, it is necessary to show a distinctive difference between observations and the expected results from SM calculations. Typically a five-sigma test is used to establish the discovery of a new particle, where the new signal must be distinguishable from the expected SM results by five standard deviations. Thus, greater precision in our understanding of the Standard Model electroweak and strong interactions will lead to a greater ability to discern new physics. These interactions form the backgrounds that must be addressed when considering measurements of new physics at the LHC.

\subsection{Overview of the Standard Model}

\subsubsection{Electroweak Gauge Bosons and their Interactions}

Electroweak theory, originally proposed by Glashow [33], Weinberg [34] and Salam [35], unified the electromagnetic and weak interactions by explaining the origin of so-called four-Fermi interactions through the mediation of a massive gauge boson.

As we now understand, electroweak interactions follow an $S U(2)_{L} \times U(1)_{Y}$ symmetry, which is gauged by four spin-1 fields: $W_{\mu}^{a}=\left(W_{\mu}^{1}, W_{\mu}^{2}, W_{\mu}^{3}\right)$ gauge the $S U(2)$ and 
$B_{\mu}$ gauges the $U(1)$, with corresponding coupling strengths $g$ and $g^{\prime}$, respectively. For an unstable Higgs potential that develops a vacuum energy, this symmetry is spontaneously broken to the low energy mass eigenstates: the massless photon, $A_{\mu}$, and the massive $Z$ and $W$ bosons, $Z_{\mu}$ and $W_{\mu}^{ \pm}$. These are related to the fundamental $W_{\mu}^{a}$ and $B_{\mu}$ fields by the following relations:

$$
\begin{aligned}
A_{\mu} & \equiv W_{\mu}^{3} \sin \theta_{W}+B_{\mu} \cos \theta_{W} \\
Z_{\mu} & \equiv W_{\mu}^{3} \cos \theta_{W}-B_{\mu} \sin \theta_{W}, \\
W_{\mu}^{ \pm} & \equiv\left(W_{\mu}^{1} \mp i W_{\mu}^{2}\right) .
\end{aligned}
$$

Further details on the Higgs potential is given in the following section.

Electroweak (EW) interactions form a chiral theory, where the SM fermions are classified into chiral components - left handed fermions transform as doublets under the $S U(2)_{L}$ symmetry while right handed fermions transform as singlets. The fermion doublets and singlets are given by,

$$
\begin{array}{ll}
q_{L}^{i}=\left(\begin{array}{c}
u_{L}^{i} \\
d_{L}^{i}
\end{array}\right) & \ell_{L}^{i}=\left(\begin{array}{c}
\nu_{L}^{i} \\
e_{L}^{i}
\end{array}\right) \\
q_{R}^{i}=u_{R}^{i}, d_{R}^{i} & \ell_{R}^{i}=e_{R}^{i}
\end{array}
$$

where the superscript $i$ denotes the generation $(i=1,2,3)$.

The EW Lagrangian [36] governing the interactions of fermions and EW gauge bosons, in terms of the mass eigenstates, is given by [36]:

$$
\begin{aligned}
& \mathcal{L}_{E W}= \sum_{i} \bar{\psi}_{i}\left(i \gamma^{\mu} \partial_{\mu}-m_{i}\right) \psi_{i}+\mathcal{L}_{N C}+\mathcal{L}_{C C} \\
& \mathcal{L}_{N C}=-\sum_{j} \bar{\psi}_{j} \gamma^{\mu} A_{\mu}\left[g T_{j}^{3} \mathrm{~s}_{W}+g^{\prime} Y_{j} \mathrm{c}_{W}\right] \psi_{j} \\
& \quad-\sum_{j} \bar{\psi}_{j} \gamma^{\mu} Z_{\mu}\left[g T_{j}^{3} \mathrm{c}_{W}-g^{\prime} Y_{j} \mathrm{~s}_{W}\right] \psi_{j} \\
& \mathcal{L}_{C C}=-\sum_{i, j} \frac{g}{\sqrt{2}} \bar{\psi}_{j} \gamma^{\mu}\left(1-\gamma^{5}\right)\left[T^{+} W_{\mu}^{+}+T^{-} W_{\mu}^{-}\right] \psi_{i}
\end{aligned}
$$



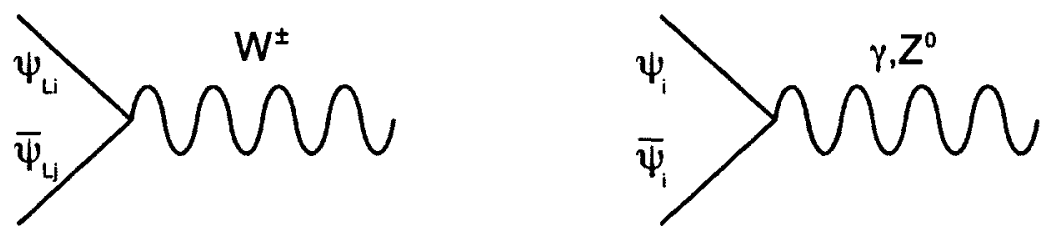

Figure 2.1: Feynman diagrams involving the interaction of fermions with electroweak gauge bosons. $\psi_{i}$ represents any chiral fermion. $\psi_{L i, j}$ represent any complementary left handed chiral fields.

Here, $Y_{j} \equiv q_{j}-T_{j}^{3}$ is the weak $U(1)_{Y}$ hypercharge for fermion flavour $j$, in terms of electromagnetic charge $q_{j}$ and the third component of the chiral isospin eigenvalue, $T_{j}^{3} . \quad T^{ \pm}$are the isospin raising/lowering operators, and the coupling strengths are related to the fundamental electric charge via the relations: $g \sin \theta_{W}=g^{\prime} \cos \theta_{W}=e$ $\left(c_{W}=\cos \theta_{W}\right.$ and $\left.s_{W}=\sin \theta_{W}\right)$. The angle $\theta_{W}$ is the weak angle, given by $\sin ^{2} \theta_{W} \equiv 1-M_{W}^{2} / M_{Z}^{2}=0.23122$ ( $\overline{\mathrm{MS}}$ scheme). The fields denoted by $\psi_{j}$ represent the fermions listed previously, and the Lagrangian subscripts NC and CC denote the neutral current and charged current respectively.

Figures 2.1 and 2.2 diagrammatically represent the interactions of fermions and gauge bosons as well as gauge-gauge interactions, via Feynman diagrams representing the possible three- and four-point interaction vertices involved in electroweak theory [37]. Any purely electroweak interaction can be described schematically by using combinations of these vertices and propagators for intermediate particle states.

\subsubsection{Electroweak Symmetry Breaking}

To explain the origin of the mass of these gauge fields, the SM electroweak Lagrangian includes a scalar Higgs field, $\varphi$, which interacts with the gauge fields as in Eq. 2.7.

$$
\mathcal{L}=\left|\left(\partial_{\mu}+\frac{i}{2}\left(g^{\prime} Y B_{\mu}^{3}+g \tau^{a} W_{\mu}^{a}\right)\right) \varphi\right|^{2}+\frac{\mu^{2}}{2} \varphi^{\dagger} \varphi-\frac{\lambda}{4}\left(\varphi^{\dagger} \varphi\right)^{2}
$$

In this form, it is clear that the four gauge fields do not have a tree level mass 

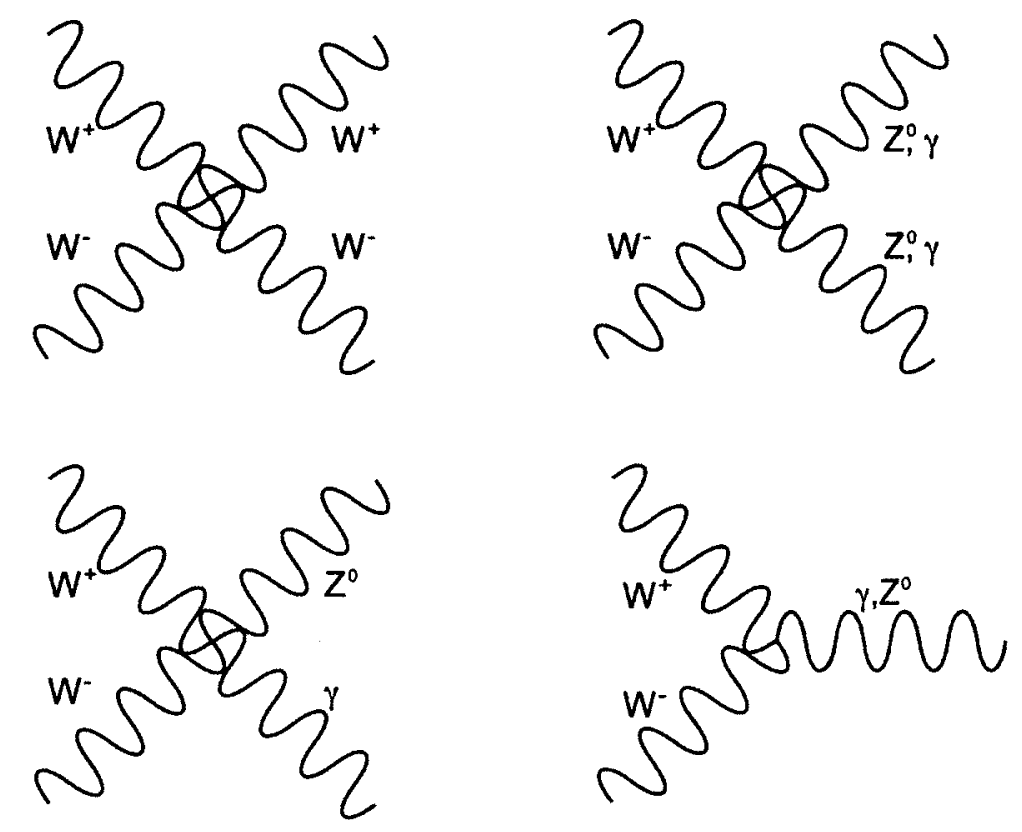

Figure 2.2: Three and four point vertices involving only electroweak gauge bosons - $W^{ \pm}, \gamma$ and $Z^{0}$.

term, and thus should be massless. However, a negative mass-squared term $\left(-\mu^{2}\right)$ for the Higgs field results in the Higgs potential being destabilized at the origin. In this situation, the Higgs potential is minimized when the Higgs field is shifted by a vacuum expectation value (vev), $v_{E W}$. When shifting $\varphi \rightarrow \varphi+v_{E W}$, the gauge fields develop mass terms corresponding to $g v_{E W}$ and $g^{\prime} v_{E W}$ from the interaction with the Higgs field in the first term in Eq. 2.7. Diagonalizing the mass matrix for the gauge fields then results in the three massive gauge bosons shown in Eq. 2.3 - the massive weak bosons, $W^{ \pm}$and $Z^{0}$, and one massless gauge boson, the photon, $A$ (or $\gamma$ ).

A Dirac Lagrangian term for a massive fermion is given by $\bar{\psi}(i \not \partial-m) \psi$, which sets the mass as an arbitrary parameter. In the SM, Dirac fermion masses are generated through Yukawa (fermion-scalar) interactions between the fermions and the scalar 
Higgs field when the Higgs develops a vev. These terms take the form of:

$$
\mathcal{L}=\sum_{i} y_{i} \bar{\psi}_{i}\left(\varphi+v_{E W}\right) \psi_{i}
$$

where the subscript $i$ refers to the specific fermion species. Thus, the Dirac mass is proportional to $y_{i} v_{E W}$.

\subsubsection{Strong Interactions}

Quantum chromodynamics (QCD) is a non-Abelian $S U(3)_{c}$ gauge theory that describes the strong force [36] that mediates the interactions between particles that have colour charge. colour charge comes in three varieties for quarks (denoted $r, g$ or $b)$ and three anti-colour varieties for anti-quarks $(\bar{r}, \bar{g}$, or $\bar{b})$. Strong interactions are mediated by the gluon, which carries both colour and anti-colour charges.

Due to the strength of the coupling, as indicated by the name, the strong force has a property known as "asymptotic freedom", where strongly interacting particles can only be treated perturbatively at high energies/short distances. At low energies/large distances, the strong coupling becomes large and perturbation theory breaks down. However, at high energy colliders like the LHC, most strong interactions can be treated perturbatively with QCD.

The self interactions of the gluons represent a major component of QCD interactions and backgrounds at the LHC and are included in the QCD Lagrangian:

$$
\begin{aligned}
\mathcal{L}_{Q C D} & =-\frac{1}{4} F_{\mu \nu}^{(a)} F^{(a) \mu \nu}+i \sum_{q} \bar{\psi}_{q}^{i} \gamma^{\mu}\left(D_{\mu}\right)_{i j} \psi_{q}^{j} \\
F_{\mu \nu}^{(a)} & =\partial_{\mu} A_{\nu}^{a}-\partial_{\nu} A_{\mu}^{a}-g_{s} f_{a b c} A_{\mu}^{b} A_{\nu}^{c} \\
\left(D_{\mu}\right)_{i j} & =\delta_{i j} \partial_{\mu}+i g_{s} \sum_{a} \frac{\lambda_{i j}^{a}}{2} A_{\mu}^{a}
\end{aligned}
$$

In this form, $\psi_{q}^{i}$ are the quark fields for colour $i$ and flavour $q, A_{\mu}^{a}$ are the gluon fields, $g_{s}$ is the strong coupling strength, and $f_{a b c}$ are the $S U(3)$ structure constants. The 


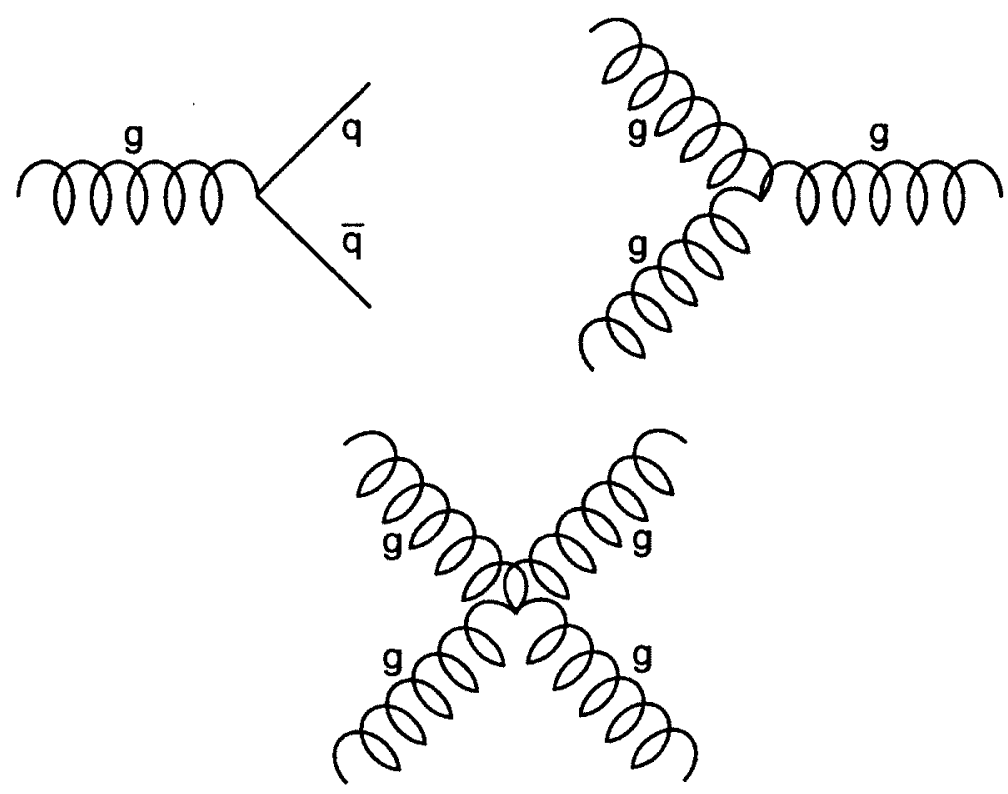

Figure 2.3: Particle interaction vertices arising from the strong interaction, including quark interactions and gluon three and four point vertices.

$3 \times 3$ Gell-Mann matrices, $\lambda_{i j}^{a}$, are the generators of the $S U(3)$ transformations. The first term represents the gluon self interactions and the second term represents the interactions of fermions with the gluons. The resulting QCD vertex diagrams are shown in Figure 2.3.

\subsubsection{Summary}

The particle content of the Standard Model is summarized in Table 2.1 in terms of mass and electric charge, and separated into families and generations. The quantum numbers of each field in the SM are given in Table 2.2, which determines the charge of each field under the appropriate symmetry transformations. The Yukawa couplings are absent from these tables, but can be determined through the relationship: $\lambda_{i}=$ $\sqrt{2} m_{i} / v_{E W}$. 
Table 2.1: A listing of the fundamental particles included in the Standard Model [36], including their hierarchy and some of their properties.

\begin{tabular}{|c|c|c|c|c|}
\hline Type & Family & Particle & Mass $(\mathrm{MeV})$ & Charge \\
\hline \multirow{12}{*}{ fermion } & \multirow{3}{*}{$\begin{array}{l}\text { u-type } \\
\text { quark }\end{array}$} & up $(u)$ & $1.7-3.1$ & $+2 / 3$ \\
\hline & & $\operatorname{charm}(c)$ & $1290_{-110}^{+50}$ & $+2 / 3$ \\
\hline & & top $(t)$ & $172900 \pm 110$ & $+2 / 3$ \\
\hline & \multirow{3}{*}{$\begin{array}{l}\text { d-type } \\
\text { quark }\end{array}$} & down $(d)$ & $4.1-5.7$ & $-1 / 3$ \\
\hline & & strange $(s)$ & $100_{-20}^{+30}$ & $-1 / 3$ \\
\hline & & bottom $(b)$ & $4190_{-60}^{+180}$ & $-1 / 3$ \\
\hline & \multirow{3}{*}{$\begin{array}{l}\text { e-type } \\
\text { lepton }\end{array}$} & electron $(e)$ & 0.511 & -1 \\
\hline & & muon $(\mu)$ & 105.66 & -1 \\
\hline & & $\operatorname{tau}(\tau)$ & $1776.82 \pm 0.16$ & -1 \\
\hline & \multirow{3}{*}{$\begin{array}{l}\nu \text {-type } \\
\text { lepton }\end{array}$} & electron neutrino $\left(\nu_{e}\right)$ & $<2 \times 10^{-6}$ & 0 \\
\hline & & muon neutrino $\left(\nu_{\mu}\right)$ & $<0.19$ & 0 \\
\hline & & tau neutrino $\left(\nu_{\tau}\right)$ & $<18.2$ & 0 \\
\hline Type & Force & Particle & Mass $(\mathrm{GeV})$ & Charge \\
\hline \multirow{5}{*}{ boson } & strong & gluon $(g)$ & $0^{1}$ & 0 \\
\hline & electric & photon $(\gamma)$ & $<6 \times 10^{-26}$ & 0 \\
\hline & weak & Z-boson $(Z)$ & $91.1876 \pm 0.0021$ & 0 \\
\hline & weak & W-boson $\left(W^{ \pm}\right)$ & $80.399 \pm 0.023$ & \pm 1 \\
\hline & $\mathrm{n} / \mathrm{a}$ & Higgs boson $(H)$ & $>114.4$ & 0 \\
\hline
\end{tabular}

1 This is an assumed value. Experiment does not preclude a mass of less than a few $\mathrm{MeV}$. 
Table 2.2: Particle quantum numbers in the SM.

\begin{tabular}{|c|c|c|c|}
\hline Field & $S U(3)_{c}$ & $S U(2)_{L}$ & $U(1)_{Y}$ \\
\hline$u_{L}$ & 3 & 2 & $1 / 6$ \\
$d_{L}$ & 3 & 2 & $1 / 6$ \\
$u_{R}$ & 3 & 1 & $2 / 3$ \\
$d_{R}$ & 3 & 1 & $-1 / 3$ \\
$e_{L}$ & 1 & 2 & $-1 / 2$ \\
$\nu_{L}$ & 1 & 2 & $-1 / 2$ \\
$e_{R}$ & 1 & 1 & -1 \\
$g$ & 8 & 1 & 0 \\
$W$ & 1 & 3 & 0 \\
$B$ & 1 & 1 & 0 \\
$\varphi$ & 1 & 2 & $1 / 2$ \\
\hline
\end{tabular}

\subsection{Physics Beyond the Standard Model}

As mentioned, there are many reasons to believe that there is new physics at energy scales above those to which the SM has currently been tested. Furthermore, there is evidence to suggest that this new physics should arise at the $\mathrm{TeV}$ scale. The latest high energy collider, the Large Hadron Collider, is designed to specifically search for this new physics by significantly expanding the high energy frontier.

\subsubsection{Indication for Physics at the TeV Scale}

Constraints on new physics arise from precision EW measurements, from measurements of meson properties at meson factories, and from direct measurements at high energy colliders such as the Tevatron and the LHC. Precision constraints are determined by examining the effects of new physics, such as higher-dimension operators that are suppressed by the scale of the new physics, on precisely measured parameters. 
For example, any higher dimension operators that violate custodial $S U(2)$ symmetry (such as $\left(h^{\dagger} D_{\mu} h\right)^{2} / \Lambda^{2}$ ) suggest that the scale $(\Lambda)$ for new strongly interacting physics must be constrained to $\Lambda>10 \mathrm{TeV}$.

But this does not preclude the possibility of weakly interacting physics at lower scales. Loops involving Higgs self-interactions, gauge bosons, and fermions, as shown in Fig. 2.4, result in corrections to the mass of the Higgs boson. The top quark loop contribution, given by

$$
\begin{aligned}
\delta m_{h}^{2} & =\int \frac{d^{4} p}{(2 \pi)^{4}}(-1) N_{c} \operatorname{Tr}\left[i \lambda_{t} \frac{i}{\not p} i \lambda_{t} \frac{i}{\not p}\right] \\
& =-\frac{4 N_{c} \lambda_{t}^{2}}{(2 \pi)^{2}} \int \frac{d^{4} p}{p^{2}} \\
& =\frac{-3}{8 \pi^{2}} \lambda_{t}^{2} \Lambda^{2}
\end{aligned}
$$

results in a divergent mass term that scales with $\Lambda$ unbounded unless there is some physical or dynamic reason why the scale $\Lambda$ is cut off at some point. Measurements from the LEP collaboration [38] restrict the mass of the Higgs boson to below about $200 \mathrm{GeV}$, which clearly indicates that the Higgs mass is not divergent, and thus there must be some cancellation occurring that keeps the Higgs mass at the weak scale.

These divergent corrections are typically assumed to cut off at scale $\Lambda$, where $\Lambda$ represents the approximate scale at which new physics beyond the SM result in a cancellation. Without such a cancellation from new physics, these parameters would necessarily be fine tuned to provide a precise cancellation, from the Planck scale down to the EW scale, in order to result in a Higgs mass as indicated by LEP. This is known as the hierarchy or fine-tuning problem. Calculations of the loop contributions from the top sector, as in Eq. 2.10, as well as the gauge and scalar sectors, indicate that the scale at which new physics must arise to cancel these loops is around 1-2 TeV. 

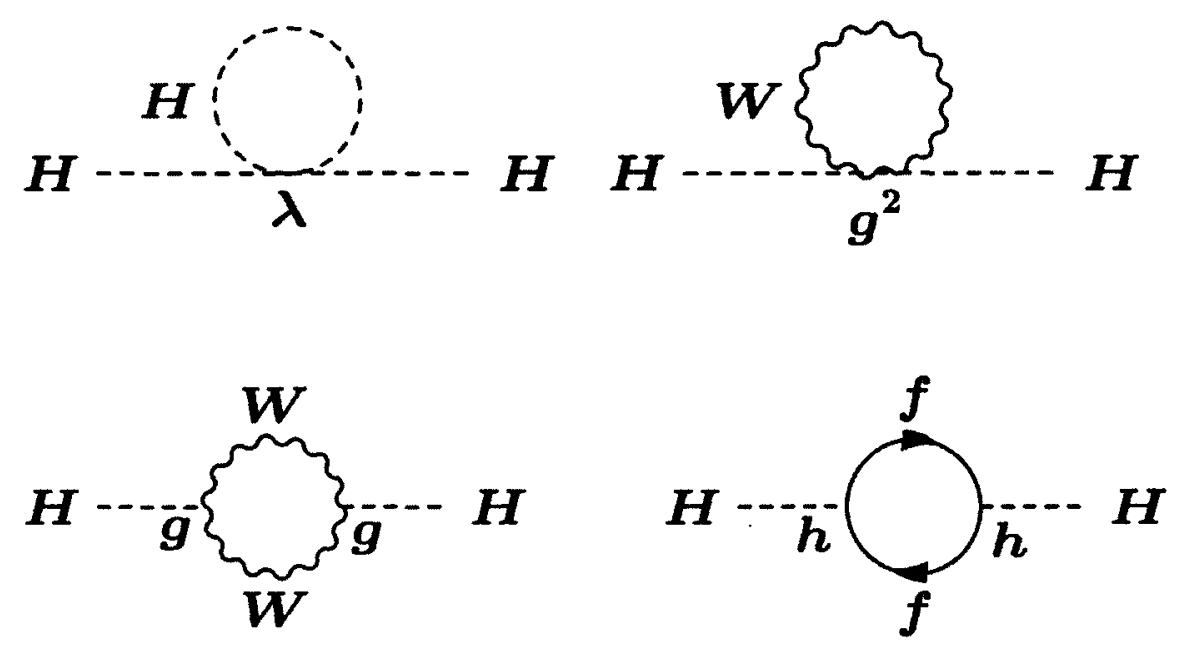

Figure 2.4: One loop divergent contributions to the Higgs mass.

\subsubsection{The Large Hadron Collider}

The Large Hadron Collider (LHC) is built in the former tunnels of the LEP collider at the CERN facility in Geneva, Switzerland, and is a proton-proton collider designed to achieve energies of $14 \mathrm{TeV}$. Due to technical difficulties, the $\mathrm{LHC}$ ran at $7 \mathrm{TeV}$ for two years, between 2010 and 2011, and is currently running at $8 \mathrm{TeV}$ for 2012, with the intent to shut down in 2013 to increase the energy for 2014.

Since the colliding protons in the LHC are hadrons composed of quarks and gluons, the energy of each individual interacting parton (quarks and gluons) in any collision cannot be controlled. Instead, collision energies depend on the fraction of momentum that is carried by the interacting parton, which is statistically described by the parton distribution functions (pdf). It is for this reason that the LHC is sometimes referred to as a "discovery machine" - it can push the energy frontier, but precise measurements are more challenging than at a lepton (electron) collider due to the inability to control the energy of each collision.

The LHC has two primary detector experiments for high energy collisions - A Large 
Toroidal LHC Apparatus (ATLAS) [39] and the Compact Muon Solenoid (CMS) [40]. The capabilities and specializations of these two detectors differ slightly, but both are capable of performing the same measurements and thus confirm any results or discoveries made. Details of the capability of these detector experiments are crucial to predicting the sensitivity of the LHC to new physics and will be discussed throughout this thesis, in reference to assessments of the viability of phenomenological studies of new particle interactions. 


\section{Part I}

\section{Examining Non-Universal Extra}

Neutral Gauge Bosons at the LHC 


\section{Chapter 3}

\section{Extra Neutral Gauge Bosons}

\subsection{Motivation}

Extra neutral gauge bosons $\left(Z^{\prime}\right)[9-12]$ are common in models of BSM physics, as they arise in nearly every scenario that includes an expanded weak sector. To a theorist, a $Z^{\prime}$ is considered a neutral (charge 0 ), spin-1, colourless, self-adjoint state (like the $Z_{0}$, it is its own antiparticle). To an experimentalist at the LHC, a $Z^{\prime}$ is a resonance produced via the Drell-Yan $\left(p p \rightarrow \ell^{+} \ell^{-}+X\right.$, where $\left.\ell=e, \mu\right)$ process, with an angular distribution of decay products that indicates a spin-1 state. While a $Z^{\prime}$ can decay to quarks, large QCD backgrounds make it an unattractive channel for discovery, and thus lepton pairs are considered a more attractive signature.

While the LHC will be able to push the discovery reach for these types of resonances to very high energies [41], understanding the properties of a neutral resonance will be crucial to determining its origin. Other possible new states may share similar production and decay modes, such as a spin-0 $\tilde{\nu}$ (sneutrino) in R-parity violating SUSY [42,43] or a spin-2 Kaluza-Klein excitation in Randall-Sundrum models [44-46]. For this reason, determining the spin is one of the first and most important measurements for a Drell-Yan resonance. A number of observables have been designed to elucidate the spin and the couplings to other SM particles. While the spin can 
be established by examining the angular distribution, or measuring the centre-edge asymmetry, determining the fermion couplings is more challenging.

Since the fermion couplings are not immediately apparent upon discovery of a new resonance, it is critical to find measurements that are sensitive to these values. A number of observables have been developed and explored in the literature that can provide insight into these couplings, which are based on measurements of lepton final states, including: the total width, the total cross section, the forward-backward asymmetry, the rapidity ratio [47], the off-peak asymmetry, the rare decay ratio [48], and the associated production ratio [49]. However, it is clear that these measurements are unable to isolate individual couplings due to the nature of the hadron collider. The energy and polarization of the interacting partons in the proton-proton collisions cannot be controlled, and therefore the underlying collision process can only be determined based on a probability of being produced from a variety of possible physical interactions ( $u \bar{u}$ annihilation and $d \bar{d}$ annihilation are just two possibilities).

Many studies of $Z^{\prime}$ physics focus on grand unified theories (GUT), like those based on the E6 symmetry [50-54] or the Left-Right Symmetric model [55-58] that include the possibility of a new gauge boson near the $\mathrm{TeV}$ scale, which can be well understood through measurements based on lepton final states.

There are a number of models of new physics that include a $Z^{\prime}$ with a natural suppression of decays to $e$ and $\mu$ pairs, the typical discovery modes, while boosting decay rates of hadronic final states [13-29]. These models are collectively known as non-universal models, and can have either family or generation dependent couplings. The capability of the detectors at the LHC to distinguish properties of these models has not been fully explored in the literature, as these models contain an extra parameter related to the degree of non-universality in the couplings that is fundamentally inaccessible with most traditional observables involving only decays to $e$ and $\mu$ pairs. To fully explore models with non-universal couplings at the LHC, measurements of 
other final states will be critical.

In order to determine as much information about the individual couplings as possible, several parameterizations have been developed. Since many observables are written in terms of ratios of different quantities, one parameterization [49] involves the ratios of squared couplings, which is insensitive to the overall sign of the coupling (positive/negative):

$$
\begin{aligned}
\gamma_{L}^{\ell} & =\frac{L_{\ell}^{2}}{L_{\ell}^{2}+R_{\ell}^{2}} \\
\gamma_{L}^{q} & =\frac{L_{q}^{2}}{L_{\ell}^{2}+R_{\ell}^{2}} \\
U & =\frac{R_{u}^{2}}{L_{q}^{2}} \\
D & =\frac{R_{d}^{2}}{L_{q}^{2}}
\end{aligned}
$$

where $L_{\ell}=L_{e}=L_{\nu}$ and $L_{q}=L_{u}=L_{d}$, and $L_{i}$ and $R_{i}$ represent the left handed and right handed couplings to fermion species $i$. Each of the measurable observables at the LHC can be rewritten in terms of combinations of these quantities.

Another parameterization, developed specifically for the analysis by Petriello and Quackenbush [59], considers the sum and difference between squares of couplings, such that:

$$
\begin{aligned}
& c_{u}=\left(L_{q}^{2}+R_{u}^{2}\right)\left(L_{\ell}^{2}+R_{e}^{2}\right), \\
& c_{d}=\left(L_{q}^{2}+R_{d}^{2}\right)\left(L_{\ell}^{2}+R_{e}^{2}\right), \\
& e_{u}=\left(L_{q}^{2}-R_{u}^{2}\right)\left(L_{\ell}^{2}-R_{e}^{2}\right), \\
& e_{d}=\left(L_{q}^{2}-R_{d}^{2}\right)\left(L_{\ell}^{2}-R_{e}^{2}\right) .
\end{aligned}
$$


By using Monte Carlo methods to integrate the phase space contribution to LHC observables, the values of these parameters can be determined from cross section measurements in a model independent way.

However, there are three complications with these techniques, independent of the parameterization method:

1. While there are five unique couplings $\left(L_{q}, R_{u}, R_{d}, L_{\ell}, R_{e}\right)$, LHC observables cannot distinguish between all five. As a result, all LHC observables can be written in terms of only four parameters. [12]

2. All couplings of fermions to a $Z^{\prime}$ enter into the cross section formulae as squares. It is not possible to determine the overall sign of any individual coupling.

3. These parameterizations assume that the couplings within fermion species are identical between generations. Since most models have generation universal couplings, and observables discussed in the literature do not focus on decays to third generation final states, this issue has been overlooked.

Non-universal couplings are therefore not apparent through measurements that can be written in terms of either of these parameterization schemes. In such scenarios, there are two separate symmetry groups under which fermions may transform and thus two separate overall gauge coupling strengths, $g_{A}$ and $g_{B}$ for symmetries $A$ and $B$. A further parameter must be introduced as the mixing of these two coupling strengths, $\tan \phi=g_{A} / g_{B}$, to fully describe the $Z^{\prime}$, which must be tested at the LHC.

Previous work on this subject $[2,41,60]$ found that it may be possible to use the capability of the detectors at the LHC to distinguish third generation final states $(\tau$, $b$ and $t$ ) to measure these decay modes of a $Z^{\prime}$. Furthermore, measurements of decays to these final states can be used to distinguish not only whether the $Z^{\prime}$ couples in a universal fashion but also the degree of non-universality, should it be observed. This thesis examines the viability of these measurements at the LHC. 


\subsection{Models}

The models examined in this thesis comprise two groupings - those with universal coupling strengths, and those with non-universal coupling strengths. Universal $Z^{\prime}$ models include the grand unification inspired $E 6(\psi, \chi$, and $\eta$ benchmark models) $[54,61]$ and Left-Right Symmetric model variants (Left-Right and Alternate LeftRight) [12,62]; Little Higgs inspired models [30-32] (Littlest Higgs [32,63], Simplest Little Higgs [64-66], and Anomaly Free Simple Little Higgs [63,67]); 3-3-1 models based on expanding the electroweak gauge group [68,69]; and the Sequential Standard Model (a duplicate of the SM $Z$ boson, with a higher mass). Details of the $Z^{\prime}$ couplings for these models are covered in Appendix A, and are examined in greater detail in the literature.

Models with non-universal couplings are generally not included in larger $Z^{\prime}$ studies, and are therefore a focus of this thesis. As such, the relevant model details are discussed in greater detail below. The three primary non-universal models examined, as representative of the property of non-universality, are: the Ununified model (UUM), an Extended Technicolour (ETC) model and a Topcolour Assisted Technicolour (TC2) model. The UUM includes a $Z^{\prime}$ coupling strength that is dependent on the fermion family - quarks versus leptons, while both the ETC and the TC2 models instead have generation dependent coupling strengths.

\subsubsection{Ununified Model (UUM)}

The Ununified Model $[28,29]$ is based on an $S U(2)_{q} \times S U(2)_{l} \times U(1)_{Y}$ symmetry, which breaks to the SM $S U(2)_{L}$ and a custodial, high energy $S U(2)$ below some scale $\Lambda$. Left handed fermions transform as doublets under their respective $S U(2)_{i}$ groups $(i=q, l$, indices represent whether quarks or leptons are charged under the symmetry group), and singlets under opposite groups. Right handed fermions transform as singlets 
under both $S U(2)$ groups, and hypercharge assignments are the same as in the SM.

After spontaneous symmetry breaking, the mass eigenstates for the gauge bosons include a massless photon and two massive $Z$ bosons. The heavy boson then couples to the lepton and quark sectors as:

$$
g_{Z^{\prime}}=g_{Z^{0}} c_{w}\left(T_{3 q} \cot \phi-\tan \phi T_{3 l}\right)
$$

where $\phi$ represents the mixing angle characterizing the gauge couplings for the quark and lepton $S U(2)$ groups. Small values of $\sin \phi$ result in an enhanced coupling strength to quarks versus leptons.

\subsubsection{Extended Technicolour (ETC)}

Extended technicolour $[24,25]$ models typically follow an extended gauge structure of $S U(2)_{h} \times S U(2)_{l}$, where the left handed component of the lighter two generations of fermions transform under the $S U(2)_{l}$ and the third generation transforms under the $S U(2)_{h}$. Left handed fermions transform as singlets under opposite symmetries, as do the right handed fermion singlets. Hypercharge assignments are the same as in the SM.

After spontaneous symmetry breaking, the mass eigenstates for the gauge bosons include a massless photon, a SM $Z^{0}$, and a heavy $Z^{\prime}$ that couples only to left handed fermions with an enhanced coupling strength to third generation fermions. The couplings are given by:

$$
g_{Z^{\prime}}=g_{Z^{\circ}} c_{w}\left(T_{3 l} \cot \phi-\tan \phi T_{3 h}\right)
$$

where $\phi$ represents the mixing angle characterizing the gauge couplings for the two $S U(2)$ groups. Large values of $\sin \phi$ result in an enhanced coupling strength to third generation fermions. 


\subsubsection{Topcolour Assisted Technicolour (TC2)}

Topcolour assisted technicolour [13-18] extends the electroweak sector with a $U(1)_{h} \times$ $U(1)_{l}$ that breaks to the SM hypercharge $U(1)_{Y}$ and a residual, high scale $U(1)^{\prime}$. The third generation fermions transform under the $U(1)_{h}$ group while the first two generation of fermions transform under the $U(1)_{l}$ group, with hypercharge assignments that are the same as in the SM.

In this type of model, the $Z^{\prime}$ plays a role in the generation of the large top quark mass by providing a tilting mechanism for the top quark seesaw. After symmetry breaking, the $Z^{\prime}$ couplings are given by:

$$
\begin{aligned}
g_{Z^{\prime}}^{3} & =\frac{1}{2} g_{Z^{0}} s_{w} Y_{S M} \cot \phi \\
g_{Z^{\prime}}^{1,2} & =\frac{1}{2} g_{Z^{0} s_{w}} Y_{S M} \tan \phi
\end{aligned}
$$

where $\phi$ represents the mixing angle characterizing the gauge couplings for the two $U(1)$ groups, and the superscripts refer to the fermion generation for the coupling. Small values of $\sin \phi$ result in a larger coupling of the $Z^{\prime}$ to third generation fermions. 


\section{Chapter 4}

\section{Calculations}

\subsection{Neutral Gauge Bosons}

At hadron colliders, a $Z^{\prime}$ has the highest probability of being produced through the annihilation of a high energy quark - anti-quark pair, and decays rapidly to fermion anti-fermion pairs, as represented in Fig. 4.1. This is known as the Drell-Yan process. Due to the small backgrounds involved, the discovery mode at the LHC is typically considered as an excess of events within a small invariant mass range in the production of lepton pairs. For leptophobic models, the decays to heavy quarks ( $b$ and $t$ ) are possible direct discovery modes $[70,71]$ but suffer from large QCD backgrounds.

The leading order Drell-Yan process can be described mathematically as follows:

$$
\begin{aligned}
\frac{d \sigma}{d \cos \hat{\theta}}\left(p p \rightarrow Z^{\prime}, Z^{0}, \gamma \rightarrow f \bar{f}\right) & =\sum_{q} \int d x_{a} d x_{b} f_{q}\left(x_{a}, Q^{2}\right) f_{\bar{q}}\left(x_{b}, Q^{2}\right) \frac{d \hat{\sigma}(\hat{\theta})}{d \cos \hat{\theta}} \\
& +f_{\bar{q}}\left(x_{a}, Q^{2}\right) f_{q}\left(x_{b}, Q^{2}\right) \frac{d \hat{\sigma}(\pi-\hat{\theta})}{d \cos \hat{\theta}}
\end{aligned}
$$

where $d \hat{\sigma} / d \cos \hat{\theta}$ is given by

$$
\frac{d \hat{\sigma}}{d \cos \hat{\theta}}\left(q \bar{q} \rightarrow Z^{\prime}, Z^{0}, \gamma \rightarrow f \hat{f}\right)=\frac{\pi \alpha_{e m}^{2} N_{c} \beta_{f}}{8 c_{W}^{4} s_{W}^{4} \hat{s}}\left\{\left(1+\beta_{f}^{2} \cos ^{2} \hat{\theta}\right) S_{q}+2 \beta_{f} \cos \hat{\theta} A_{q}+S_{q}^{\prime}\right\}
$$




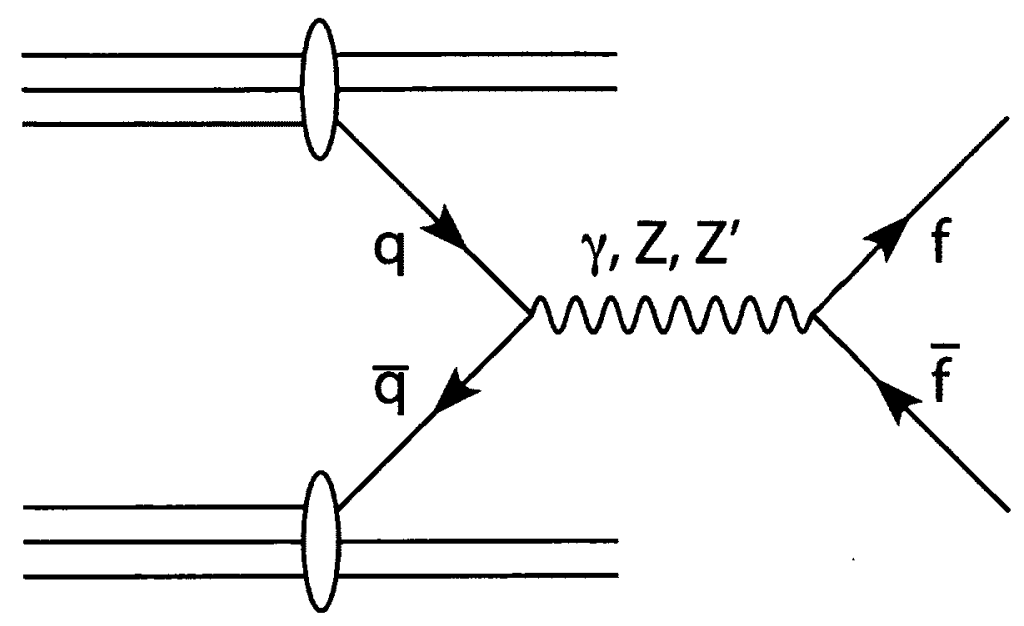

Figure 4.1: Feynman diagram representing the Drell-Yan production of leptons at a hadron collider.

and

$$
\begin{aligned}
S_{q}, A_{q} & =\sum_{i, j=\gamma, Z, Z^{\prime}}\left(\frac{\hat{s}}{\hat{s}-M_{i}^{2}-i \Gamma_{i} M_{i}}\right)\left(\frac{\hat{s}}{\hat{s}-M_{j}^{2}+i \Gamma_{j} M_{j}}\right) \\
& \times\left(R_{f}^{i} R_{f}^{j} \pm L_{f}^{i} L_{f}^{j}\right)\left(R_{q}^{i} R_{q}^{j} \pm L_{q}^{i} L_{q}^{j}\right) \\
S_{q}^{\prime} & =\sum_{i, j=\gamma, Z, Z^{\prime}}\left(\frac{4 m_{f}^{2}}{\hat{s}}\right)\left(\frac{\hat{s}}{\hat{s}-M_{i}^{2}-i \Gamma_{i} M_{i}}\right)\left(\frac{\hat{s}}{\hat{s}-M_{j}^{2}+i \Gamma_{j} M_{j}}\right) \\
& \times\left(R_{f}^{i} L_{f}^{j}+L_{f}^{i} R_{f}^{j}\right)\left(R_{q}^{i} R_{q}^{j}+L_{q}^{i} L_{q}^{j}\right) .
\end{aligned}
$$

In Eq 4.1 and $4.2, M_{i}$ and $\Gamma_{i}$ are the masses and width of the photon, SM $Z^{0}$ and $Z^{\prime} ; L_{f}^{i}$ and $R_{f}^{i}$ are the left and right handed couplings of the gauge bosons to fermion species $f ; m_{f}$ is the mass of the final state fermion; $f_{q, \bar{q}}\left(x, Q^{2}\right)$ are the parton distribution functions (pdf); $Q^{2}$ is the scale at which the parton distribution functions are evaluated, which is taken to be $\hat{s}$ - the square of the parton centre-of-mass energy; $\hat{\theta}$ is the centre-of-mass scattering angle defined by the direction of the interacting quark; $N_{c}$ is a colour factor based on the final state, which has a value of 3 for quarks and 1 for leptons; and $\beta_{f}=\sqrt{1-4 m_{f}^{2} / \hat{s}}$ is a kinematic factor to account for the mass of the final state fermions. In my calculations, I used $\alpha_{e m}=1 / 127.9, \sin ^{2} \theta_{w}=0.231$, 
$M_{Z}=91.188 \mathrm{GeV}, \Gamma_{Z}=2.495 \mathrm{GeV}$ and $m_{t}=171.2 \mathrm{GeV}$. The parton distribution function (pdf) used was the CTEQ6M set [72] for calculating the momentum fraction of the interacting partons. The $Z^{\prime}$ width is calculated assuming only decays to SM fermions and neglecting $Z-Z^{\prime}$ mixing, as well as neglecting decays to pairs of $W^{ \pm}$ and $Z^{0}$ gauge bosons.

The total $Z^{\prime}$ decay width is given by the sum of the partial widths, $\Gamma_{Z^{\prime}}=\sum_{f} \Gamma_{f}$, where the partial widths are given by:

$$
\Gamma\left(Z^{\prime} \rightarrow f \bar{f}\right)=\frac{N_{c} \alpha_{e m} M_{Z^{\prime}} \beta_{f}}{6 c_{w}^{2} s_{w}^{2}}\left(\left(R_{f}^{2}+L_{f}^{2}\right)\left(1-\frac{m_{f}^{2}}{M_{Z^{\prime}}^{2}}\right)+6 L_{f} R_{f} \frac{m_{f}^{2}}{M_{Z^{\prime}}^{2}}\right),
$$

The last term, proportional to the ratio of the decay product mass over the $Z^{\prime}$ mass is only significant for top quarks, assuming the $Z^{\prime}$ mass is relatively light. For a $Z^{\prime}$ mass of $2 \mathrm{TeV}$, this term is suppressed by more than $1 / 100$. The same suppression is present for the $S_{q}^{\prime}$ term in the differential cross section.

To account for next-to-leading-order (NLO) effects in the cross section, a K-factor is included [73]. The K-factor included with the signal cross section accounts for soft, collinear initial state radiation effects in addition to virtual gluon loops, as these must be considered together in order to remove the infrared singularities [73]. Next-tonext-to-leading-order (NNLO) corrections are not included as uncertainties in parton distribution functions dominate over such small effects [74,75]. Both QCD [76-78] and EW [79-81] corrections are included in the width calculation, however the EW corrections were found to be a minor contribution.

Phase space integration of Eq. 4.2 was performed using Monte-Carlo integration methods with weighted events, including full interference between the $Z^{\prime}$ production and EW backgrounds. Kinematic cuts were implemented within the integration. Similarly, phase space integration for the QCD backgrounds for hadronic final states was performed using Monte-Carlo integration following the distributions in Barger and Phillips [82], and compared against WHiZaRD (with O'MEGA matrix 
elements) [83-85] and MADGRAPH [86] Monte-Carlo event generators. All three background calculations agreed.

Higher order effects are not accounted for in the calculation of the backgrounds. These effects are highly dependent on the energy of the event, such that $\sigma(\mathrm{NLO}) / \sigma(\mathrm{LO}) \approx 2$ at low energy, but $\sigma(\mathrm{NLO}) / \sigma(\mathrm{LO}) \approx 0.3-0.4$ for $p_{T}(t \bar{t})>$ $100 \mathrm{GeV}[87]$. Instead of accounting for this directly, the effects were approximated by varying the backgrounds by up to a factor of 2 (i.e. $\sigma_{\mathrm{QCD}}(t \bar{t}) \rightarrow 2.0 \times \sigma_{\mathrm{QCD}}(t \bar{t})$ ) to observe how this changed the results. Larger backgrounds resulted in poorer statistical uncertainties, as expected, but had a smaller effect on models with preferential couplings to third generation final states, which is the focus of this study. Additionally, the increase in statistical uncertainty can be overcome through higher luminosity studies, which the LHC may perform.

\subsection{Observables}

In principle, observables can be defined based on various integrations over the decay angle, $\hat{\theta}$, as these regions provide different weightings for the combination of initial state couplings. In practice, events in ATLAS [39] and CMS [40] are categorized based on the energy, transverse momentum, and pseudorapidity of the decay products, and the decay angle must be reconstructed from these values. If the four momentum of the decay products is given by $p_{f}=\left(E_{f}, p_{x}, p_{y}, p_{z}\right)$ and $p_{\bar{f}}=\left(E_{\bar{f}}, p_{\bar{x}}, p_{\bar{y}}, p_{\bar{z}}\right)$, then the common experimental variables are defined as:

$$
\begin{array}{rc}
\text { Energy : } & E=E_{f} \\
\text { Transverse Momentum : } & p_{T}=\sqrt{p_{x}^{2}+p_{y}^{2}}=\sqrt{p_{\bar{x}}^{2}+p_{\bar{y}}^{2}} \\
\text { Rapidity : } & y=\frac{1}{2} \ln \frac{E+p_{z}}{E-p_{z}} \\
\text { Pseudorapidity : } & \eta=\frac{1}{2} \ln \frac{|\bar{p}|+p_{z}}{|\bar{p}|-p_{z}}
\end{array}
$$




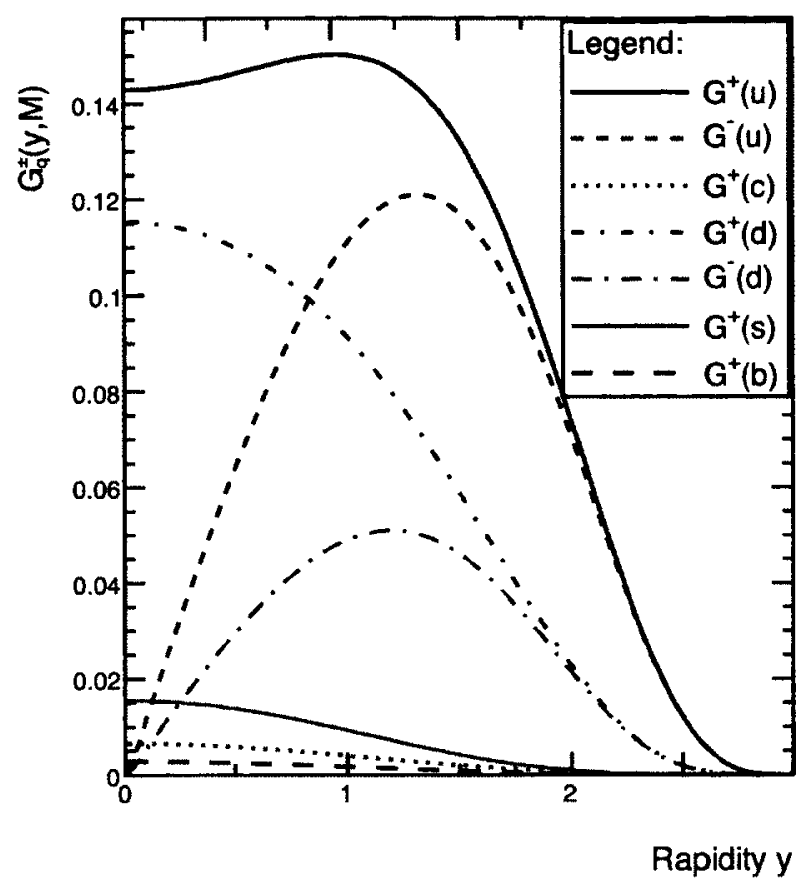

Figure 4.2: Symmetric and anti-symmetric pdf combinations versus $Z^{\prime}$ rapidity, showing the relative contributions to symmetric and anti-symmetric decays.

where $|\bar{p}|=\sqrt{p_{x}^{2}+p_{y}^{2}+p_{z}^{2}}$.

The decay angle is measured relative to the direction of the momentum of the interacting quark. Since the momentum of each proton at the LHC is carried by both quarks and anti-quarks, it is impossible to discern which proton the interacting quark originated from, and so the decay angle must be loosely approximated as referenced to the boost direction of the boson. This approximation is generally valid since there is a greater probability that the momentum fraction of the interacting quark is larger than the momentum fraction of the antiquark. Thus, the $Z^{\prime}$ has a higher probability of being boosted by a higher momentum quark than anti-quark. In the region where the boson has a low boost, this approximation fails as there is an equal probability that the interacting quark came from either proton.

This is made clear by examining the symmetric and antisymmetric pdf contributions, as in Fig. 4.2, where the following notation is used: $G_{q}^{ \pm}=$ 
$x_{a} x_{b}\left(f_{q}\left(x_{a}, Q^{2}\right) f_{\bar{q}}\left(x_{b}, Q^{2}\right) \pm f_{\bar{q}}\left(x_{a}, Q^{2}\right) f_{q}\left(x_{b}, Q^{2}\right)\right)$. These are the sum and difference of the probabilities of the parton from proton $a$ (an arbitrary assignment) being a quark $(q)$ versus an anti-quark $(\bar{q})$, which can be mapped to the rapidity of the $Z^{\prime}$, $Y_{Z^{\prime}}$. It is clear from the antisymmetric combinations, $G_{q}^{-}$, that the probabilities are equal in the region of low boost, indicating the challenge in reconstructing $\theta$ versus $\pi-\theta$ in this region, where the likelihood of the $Z^{\prime}$ being boosted in the direction of the interacting quark is equally possible for either proton.

Symmetric integrations over the the decay angle are not sensitive to this problem, as the total number of events is independent of correctly identifying which proton produced the interacting quark, and give information on the sum of the square of the left and right couplings $\left(R^{2}+L^{2}\right)$. Antisymmetric integrations over the decay angle are sensitive to this problem, and give information on the difference of the square of the left and right couplings $\left(R^{2}-L^{2}\right)$. It has been proposed that excluding events with low $Z^{\prime}$ boost, where the centre-of-mass frame is close to the lab frame, can increase sensitivity to couplings in anti-symmetric integrations [59], but I found an overall increase in statistical uncertainties due to the removal of the large number of events in this region. As such, this central region is not excluded in any calculations.

\subsubsection{Centre-Edge Asymmetry}

A resonance in a dilepton final state (i.e. di-muon, di-electron) can arise from spin-0, spin- 1 , or spin- 2 bosons. The resulting angular distribution, $d \hat{\sigma} / d \cos \hat{\theta}$, is dependent on the spin of the decaying boson. This is clearly evident in Fig. 4.3 for highly idealized angular distributions. Both spin-0 and spin- 1 angular distributions are essentially model independent: a spin- 0 resonance $[42,43]$ cannot have a dependence on $\hat{z}=\cos \hat{\theta}$, while a spin-1 boson has a dependence of $\propto\left(1+\hat{z}^{2}\right)$. Spin-2 resonances [44-46,88] have a dependence up to order $\hat{z}^{4}$, with model dependent coefficients for each $\hat{z}^{i}$ term.

In my calculations, the spin- 0 resonance is represented by an R-parity violating $\tilde{\nu}$ 
with $\lambda \lambda^{\prime}=(0.05)^{2}[42,43]$, while the spin-2 resonance is represented by an RS graviton with $c=0.1$ [89]. Current experimental limits and studies on model parameters are given in Ref. [42] and [46]. For the $Z^{\prime}$ cases, the $E_{6}$ models $(\psi, \chi$ and $\eta)$ [54], the Left-Right Symmetric model (LRSM, $g_{R}=g_{L}$ ) [62], both the Littlest Higgs (LHM, $\tan \theta_{H}=1.0$ ) [32] and Simplest Little Higgs (SLHM) [66] models, and the Sequential Standard Model (SSM) are compared. Further details of all of these models are covered in Appendix A.

While a measurement of the angular distributions would uniquely identify the spin of the boson, these distributions are not directly accessible at the LHC due to the convolution of the angular cross section with the parton distribution functions (as in Eq. 4.2), detector limitations and finite statistics [1]. Instead, the distributions could be reconstructed from the properties of the decay products. However, this is tedious and the only important detail is the $\hat{z}$ dependence, rather than the full distribution itself.

The centre-edge asymmetry provides a measurement that is sensitive to the angular distribution of the resonance and looks at the difference between the number of events in the central region of $\hat{z}$ to the number in the outer region, defined by some value of $\tilde{z}$. This is given by:

$$
A_{C E}=\frac{\left(\int_{-\tilde{z}}^{\tilde{z}}-\int_{-1}^{-\tilde{z}}-\int_{\tilde{z}}^{1}\right) \frac{d \sigma}{d \hat{z}} d \hat{z}}{\int_{-1}^{1} \frac{d \sigma}{d \hat{z}} d \hat{z}},
$$

This asymmetry measurement reduces the large statistical uncertainty present in performing a fit to events spread out over a binned distribution. However, this still requires the reconstruction of $\hat{z}$ for each event, which suffers from compounding uncertainties on the event kinematics.

There exists a direct mapping between the centre-of-mass decay angle and the difference in pseudorapidity of the final state fermions. Pseudorapidity is measured 


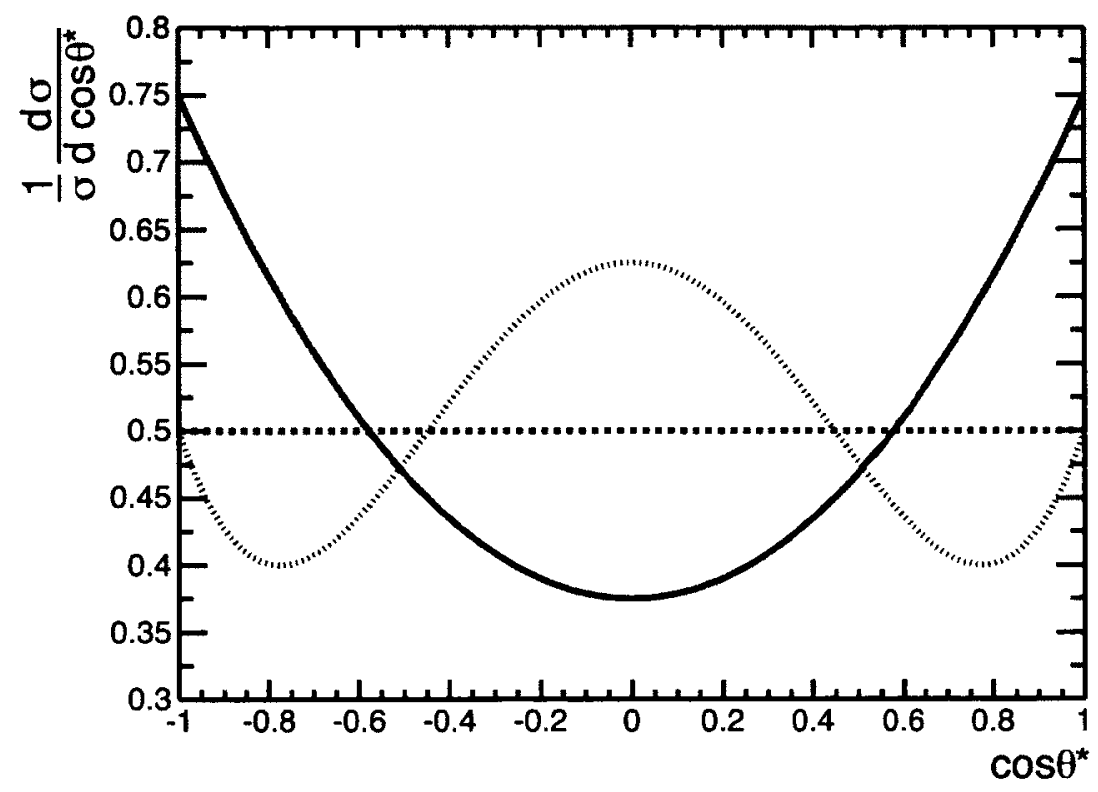

Figure 4.3: Idealized angular distribution of spin-0, spin-1 and spin-2 S-channel resonances.

directly for each event in a detector, and, since the difference in pseudorapidity is invariant under $z$-boost, measuring this difference in the lab frame is equivalent to measuring it in the centre-of-mass frame:

$$
\Delta \eta_{l a b}=\Delta \hat{\eta}=\ln \left(\frac{1+\hat{z}}{1-\hat{z}}\right)
$$

A new centre-edge asymmetry calculation, dependent on $\Delta \eta$, can be defined as:

$$
\tilde{A}_{C E}=\frac{\left(\int_{-\Pi}^{\Pi}-\int_{-\infty}^{-\Pi}-\int_{\Pi}^{\infty}\right) \frac{d \sigma}{d \Delta \eta} d \Delta \eta}{\int_{-\infty}^{\infty} \frac{d \sigma}{d \Delta \eta} d \Delta \eta}
$$

where $\Pi$ is the optimal value of $\Delta \eta$ that maximizes the distinguishability of the asymmetry. Following Osland et al., [90] this value is $\tilde{z}=0.5 \rightarrow \Pi=\Delta \eta=1.099$. While the asymmetry assumes that $\Delta \eta$ is integrable to $\pm \infty$, leptons are only distinguishable in CMS and ATLAS to within $|\eta|<2.5$, which limits the integration to $|\Delta \eta|<5$. 


\subsubsection{Forward-Backward Asymmetry}

The antisymmetric coupling combination of the $A_{q}$ term in Eq. 4.2 is accessible through the forward-backward asymmetry, $A_{F B}$. This observable is given by:

$$
A_{F B} \equiv \frac{\sigma_{F}-\sigma_{B}}{\sigma_{F}+\sigma_{B}}=\frac{\left(\int_{0}^{y_{\max }} d y \frac{d \sigma^{-}}{d y}-\int_{-y_{\max }}^{0} d y \frac{d \sigma^{-}}{d y}\right)}{\left(\int_{0}^{y_{\max }} d y \frac{d \sigma^{+}}{d y}+\int_{-y_{\max }}^{0} d y \frac{d \sigma^{+}}{d y}\right)}
$$

A simpler way to measure the forward-backward asymmetry is possible by employing pseudorapidity $(\eta)$ of the final state fermions, and is currently in use by CMS [91] and ATLAS [92] under the name forward-backward charge asymmetry. By tagging events as forward if $\left|\eta_{f}\right|>\left|\eta_{\tilde{f}}\right| \rightarrow \Delta|\eta|>0$, and backward if the reverse, then the $A_{F B}$ can be identically reconstructed, which can be seen as follows.

Assuming that the mass of the fermion is negligible $\left(m_{f} \approx 0\right)$, the momenta in the centre of mass frame is defined to be $\vec{p}_{f}=-\vec{p}_{\bar{f}}$. Thus, for $\hat{z}=\cos (\theta)$, then $p_{z}=\left|\vec{p}_{f}\right| \hat{z}$ is the longitudinal component of momentum. To boost into the lab frame, two boost variables can be defined, dependent on the momentum fractions of the interacting quarks $\left(x_{a}\right.$ and $\left.x_{b}\right), \beta=\frac{x_{a}-x_{b}}{x_{a}+x_{b}}$ and $\gamma=\left(1-\beta^{2}\right)^{-1 / 2}$, and find:

$$
\begin{aligned}
p_{f}^{\prime} & =\gamma p_{f}+\beta \gamma p_{z}=\gamma p_{f}(1+\beta z) \\
p_{f, z}^{\prime} & =\beta \gamma p_{f}+\gamma p_{z}=\gamma p_{f}(\beta+z) \\
p_{\bar{f}}^{\prime} & =\gamma p_{f}+\beta \gamma p_{z}=\gamma p_{f}(1-\beta z) \\
p_{\bar{f}, z}^{\prime} & =\beta \gamma p_{f}+\gamma p_{z}=\gamma p_{f}(\beta-z) .
\end{aligned}
$$

Pseudorapidity is defined as $\eta=\frac{1}{2} \ln \frac{|p|+p_{z}}{|p|-p_{z}}$, for $p$ taken in the lab frame, and thus the pseudorapidity of each fermion is given by:

$$
\begin{aligned}
& \eta_{f}=\frac{1}{2} \ln \frac{|1+\beta z|+\beta+z}{|1+\beta z|-\beta-z}, \\
& \eta_{\bar{f}}=\frac{1}{2} \ln \frac{|1-\beta z|+\beta-z}{|1-\beta z|-\beta+z},
\end{aligned}
$$


where the dependence on $p_{f}$ cancels out, leaving only a dependence on the decay angle and the boost. Since $|z|<1$ and $|\beta|<1$, it is clear that $(1 \pm \beta z)>0$. It can thus be shown that the pseudorapidities can be written in the form:

$$
\begin{aligned}
& \eta_{f}=\frac{1}{2} \ln \frac{1+\beta}{1-\beta} \frac{1+z}{1-z}=\frac{1}{2} \ln \frac{1+\beta}{1-\beta}+\frac{1}{2} \ln \frac{1+z}{1-z} \\
& \eta_{\bar{f}}=\frac{1}{2} \ln \frac{1+\beta}{1-\beta} \frac{1-z}{1+z}=\frac{1}{2} \ln \frac{1+\beta}{1-\beta}-\frac{1}{2} \ln \frac{1+z}{1-z}
\end{aligned}
$$

Both of these have terms of the form: $\ln \frac{1+a}{1-a}>0$ for $a>0$, and $\ln \frac{1+a}{1-a}<0$ for $a<0$. Writing these terms as $Y \equiv \frac{1}{2} \ln \frac{1+\beta}{1-\beta}$ and $Z \equiv \frac{1}{2} \ln \frac{1+z}{1-z}$, the pseudorapidity of each final state is given by:

$$
\begin{aligned}
& \eta_{f}=Y+Z \\
& \eta_{\vec{f}}=Y-Z
\end{aligned}
$$

Thus, $|Y+Z|>|Y-Z|$ when both $Y$ and $Z$ are like signed (a forward event), and $|Y+Z|<|Y-Z|$ when $Y$ and $Z$ are opposite signed (a backward event), recalling that $Y$ and $Z$ are signed the same as $y_{Z^{\prime}}$ (the $Z^{\prime}$ rapidity, or boost) and $\hat{z}$, which are the variables necessary for the traditional method of calculating the $A_{F B}$.

The $\eta$ resolution for leptons is not yet explicitly stated [93] by the experimental groups for muons or electrons, as the study is not complete. However, as an estimate, the $\tau$ candidate $\eta$ resolution is listed as $<0.04$ (with maximal values of approximately -2.5 to 2.5 for the tracking system) in preliminary results. Thus, the systematic uncertainties for correctly tagging a forward versus a backward event is dependent almost entirely on charge identification of the final states, since the region where $\Delta|\eta|$ is small also contributes nearly negligibly to the $A_{F B}$, as shown in Fig. 4.2. 
In these terms, the forward-backward asymmetry is written as:

$$
\tilde{A}_{F B}=\frac{N(\Delta|\eta|>0)-N(\Delta|\eta|<0)}{N(\Delta|\eta|>0)+N(\Delta|\eta|<0)} \approx\left(\frac{L_{f}{ }^{2}-R_{f}{ }^{2}}{{L_{f}}^{2}+{R_{f}}^{2}}\right)\left(\frac{\sum_{q} G_{q}^{-}\left(L_{q}{ }^{2}-R_{q}{ }^{2}\right)}{\sum_{q} G_{q}^{+}\left({L_{q}}^{2}+{R_{q}}^{2}\right)}\right)
$$

The approximation on the right hand side is a description of the on-peak contribution to the $\tilde{A}_{F B}$ that more clearly indicates the coupling dependencies. As before, $L_{q, f}$ and $R_{q, f}$ are the left and right handed couplings of the $Z^{\prime}$ to the final state fermion $(f)$ and initial state quarks $(q)$, and $G_{q}^{ \pm}$are the integrated symmetric and anti-symmetric combinations of the parton distribution functions that can be found from Monte-Carlo integration of the phase space.

This technique of using pseudorapidity measurements can be applied to massive final states also, though the proof is not as succinct. For hadronic final states, the $\eta$ of the centroid of the jet can be used, assuming missing transverse energy is not a significant problem. The usefulness of this technique is clear, as the recent measurement of a deviation in the forward-backward asymmetry in the $t \bar{t}$ final state at Fermilab [94] suggests that a similar measurement at the LHC would be crucial to corroborating the Fermilab results.

The ability to identify $b$ - and $t$-quarks at the LHC offers the possibility of measuring forward-backward asymmetries $\left(\tilde{A}_{F B}\right)$ in heavy quark final states to assist in the determination of individual fermion couplings to a $Z^{\prime}[1]$. At tree level, the QCD production of heavy quarks produces a fully symmetric signal, and all contributions to an $\tilde{A}_{F B}$ occur at loop level. Thus, the forward-backward asymmetry in heavy quark final states can be very sensitive to new physics. 


\subsubsection{Third Generation Ratios}

Accessing the couplings of a $Z^{\prime}$ to quarks at the LHC is generally studied by examining the relative weighting of different regions of rapidity of the $Z^{\prime}\left(y_{Z^{\prime}}\right)$, as with the forward-backward asymmetry and rapidity ratio [47] observables, or the technique proposed by Petriello and Quackenbush [59]. These assume that we have a very good understanding of the pdfs in order to determine the relative weightings of the $u \bar{u}$ and $d \bar{d}($ and $s \bar{s}$ and $c \bar{c})$ contributions.

For a $Z^{\prime}$ with a large branching ratio to quarks or third generation fermions, it may also be possible to consider a measure of an excess of events to third generation final states $\left(\tau^{+} \tau^{-}, b \bar{b}\right.$ and $\left.t \bar{t}\right)$ in order to access information on the couplings [60]. In general, the cross section dependence on couplings can be simplified with a symmetric integration over $y_{Z^{\prime}}$ and $\cos \hat{\theta}$ to find:

$$
\begin{aligned}
\sigma\left(p p \rightarrow Z^{\prime} \rightarrow f \bar{f}\right) \approx & N_{c} K_{f}\left[C_{u}\left(\left(R_{u}^{Z^{\prime}}\right)^{2}+\left(L_{u}^{Z^{\prime}}\right)^{2}\right)+C_{d}\left(\left(R_{d}^{Z^{\prime}}\right)^{2}+\left(L_{d}^{Z^{\prime}}\right)^{2}\right)\right] \\
& \times\left(\left(R_{f}^{Z^{\prime}}\right)^{2}+\left(L_{f}^{Z^{\prime}}\right)^{2}\right)
\end{aligned}
$$

where $C_{u}$ and $C_{d}$ are dependent on constants and integrations over phase space, including contributions from the pdfs, and the subscript $f$ on the couplings refers to the final state fermion. The factor of $K_{f}$ is an NLO K-factor that depends on the final state, $f$, and $N_{c}$ is a colour factor, as before. For simplicity, this assumes that the couplings for $u$ and $c$ final states are identical, and $d$ and $s$ final states are identical, and therefore $C_{u}$ and $C_{d}$ account for the like-signed contribution of the second generation sea-quark annihilations.

By taking ratios of cross sections, the dependence of the observable on the initial state $u$ and $d$ couplings, and the phase space integrations, cancel out. Thus, taking the ratio of cross sections of third generation pairs to muon pairs provides observables that are dependent only the ratio of the final state couplings, and the K-factor, while avoiding the potentially large pdf uncertainties [59]. The observables $R_{t / \mu}, R_{b / \mu}$ and 
$R_{\tau / \mu}$ are given by:

$$
\begin{aligned}
& R_{t / \mu} \equiv \frac{\sigma\left(p p \rightarrow Z^{\prime} \rightarrow t \bar{t}\right)}{\sigma\left(p p \rightarrow Z^{\prime} \rightarrow \mu^{+} \mu^{-}\right)} \approx \frac{3 K_{t}\left(L_{t}^{2}+R_{t}^{2}\right)}{\left(L_{\mu}^{2}+R_{\mu}^{2}\right)} \\
& R_{b / \mu} \equiv \frac{\sigma\left(p p \rightarrow Z^{\prime} \rightarrow b \bar{b}\right)}{\sigma\left(p p \rightarrow Z^{\prime} \rightarrow \mu^{+} \mu^{-}\right)} \approx \frac{3 K_{b}\left(L_{b}^{2}+R_{b}^{2}\right)}{\left(L_{\mu}^{2}+R_{\mu}^{2}\right)} \\
& R_{\tau / \mu} \equiv \frac{\sigma\left(p p \rightarrow Z^{\prime} \rightarrow \tau^{+} \tau^{-}\right)}{\sigma\left(p p \rightarrow Z^{\prime} \rightarrow \mu^{+} \mu^{-}\right)} \approx \frac{\left(L_{\tau}^{2}+R_{\tau}^{2}\right)}{\left(L_{\mu}^{2}+R_{\mu}^{2}\right)}
\end{aligned}
$$

where $L_{f}$ and $R_{f}$ are the left and right handed fermion couplings to the $Z^{\prime}$ and the $K$ factors incorporate both the QCD and QED NLO correction factors $[73,76]$. The factor of three in the hadronic ratios accounts for the three colours of quarks. It is clear that these observables are independent of the parton distribution functions.

For models with generation dependent couplings, all three ratios should present distinctive results. However, for models with quark-lepton dependent couplings, such as the Ununified model, only the top and bottom ratios will provide interesting results, and the tau ratio should present a value of unity, similar to all models with universal couplings. In this way, these three observables are capable of uniquely identifying the non-universal nature of couplings - either generation dependence or lepton/quark dependence.

In terms of the parameterization of couplings discussed in 3.1, the third generation quark ratios can be rewritten as:

$$
\begin{aligned}
R_{t / \mu} & \approx K \gamma_{L}^{q}(U+1) \\
R_{b / \mu} & \approx K \gamma_{L}^{q}(D+1) \\
R_{\tau / \mu} & \approx \tan ^{4} \phi(\approx 1 \text { universal })
\end{aligned}
$$

where $\gamma_{L}^{q}, U$ and $D$ are defined in Eq. 3.1. It should be noted that all other observables in the literature are unable to give information regarding the $\gamma_{L}^{q}$ parameter [12] 
or the mixing angle for models with non-universal couplings, which makes these measurement invaluable for elucidating the properties of a $Z^{\prime}$ at the LHC. For models with generation universal couplings, the $\tau$ to $\mu$ ratio should result in a value of unity.

\subsection{Identification and Backgrounds}

The ATLAS [39] and CMS [40] detectors have four layers that contribute to identification of different final states. The first layer is the tracker, which tracks the curved path of the particles due to the applied strong magnetic field. The second is an electromagnetic calorimeter, which records the energy deposited by electrons and hadrons (and photons). The entirety of the energy of relativistic electrons is expected to be deposited in the electromagnetic calorimeter, prior to reaching the third layer. The third layer is a hadronic calorimeter, which absorbs the remaining energy of hadronic jets. The final layer is a muon detector, which measures the long lived muon tracks.

To a first approximation, only five states can be identified: photons, muons, electrons, hadrons and missing transverse energy (indicating either neutrinos or new "invisible" physics). However, the decay products of the electroweak gauge bosons and third generation fermions produce distinctive signatures that can be reconstructed to a certain degree. Much effort has been devoted to establishing the best techniques to distinguish these final states in order to better understand each individual collision, as well as to understand the contributions of background processes to measurements of new physics.

From a phenomenological perspective, these can be approximated as detection efficiencies that vary based on the kinematics of the collision. The efficiencies used in this analysis are discussed in this section. 


\subsubsection{Muons and Electrons}

Muons and electrons leave very distinctive signatures in both ATLAS and CMS. Recent studies for the ATLAS working group [93] suggest an identification efficiency above $95 \%$ for muons with $p_{T}>30 \mathrm{GeV}$, and above $90 \%$ for electrons with $p_{T}>30$ $\mathrm{GeV}$. In this analysis, it is assumed that backgrounds that can fake a di-muon or di-electron signal are negligible.

\subsubsection{Bottom Quarks}

A number of $b$-tagging algorithms have been developed for ATLAS and commissioned with the 2011 dataset. The most promising methods are based on the IP3D algorithm, which employs a likelihood ratio technique that compares actual data to Monte Carlo simulations. The IP3D+JetFitter algorithm is typically quoted in experimental papers, as it provides the best rejection against light jets, and gives an efficiency of $\epsilon_{b}=70 \%$ with only $\epsilon_{j, m i s t a g}=1 \%$ [95] of light jet events faking a b-jet. It is possible to increase the rejection of light jet events by tightening the requirements in the tagging algorithm, and thus similarly reducing the tagging efficiency. An order of magnitude improvement in the rejection rate corresponds to an approximately $20 \%$ decrease in tagging efficiency.

These predictions come from examinations of $t \bar{t}$ events, and are valid up to a transverse momentum of $p_{T, j e t} \approx 400 \mathrm{GeV}$. The rejection against light jet events drops as the energy of the jet increases, which can be offset by employing tighter tagging. Prior to comparison against real data, the tagging efficiency was typically cited as $\epsilon_{b}=60 \%$ with $\epsilon_{j, m i s t a g}=1 \%$ [93].

In the study reported in this thesis, I assume a tagging efficiency of $\epsilon_{b}=60 \%$ for each $b$ quark with $\epsilon_{j, \text { mistag }}=1 \%$, as these are the values that were published at the time of the original study [2]. QCD production of $b \bar{b}$ events are the primary 
irreducible background, with dijets forming the primary reducible background.

\subsubsection{Top Quarks}

Top quarks decay approximately $100 \%$ of the time to a $W^{+} b$ final state. However, the on-shell $W$ decays only $67.6 \%$ of the time to hadrons and $21.3 \%$ of the time to $\ell^{+} \nu$ (where $\ell=e, \mu$ ). In $t \bar{t}$ final states from $Z^{\prime}$ decays, three decay modes are typically considered: fully leptonic $\left(t \bar{t} \rightarrow W^{+} W^{-} b \bar{b} \rightarrow \ell^{+} \nu_{\ell} \ell^{-} \overline{\nu_{\ell}} b \bar{b}\right)$, with a combined branching ratio of $4.5 \%$; semi-leptonic $\left(t \bar{t} \rightarrow W^{+} W^{-} b \bar{b} \rightarrow \ell^{+} \nu_{\ell} j j b \bar{b}\right)$, with a branching ratio of $28.8 \%$; and fully hadronic $\left(t \bar{t} \rightarrow W^{+} W^{-} b \bar{b} \rightarrow j j j j b \bar{b}\right)$, with a branching ratio of $47.5 \%$.

The fully and semi-leptonic modes have the benefit of avoiding purely QCD backgrounds, but suffer from missing information about the event through missing transverse energy, $E_{T}$. The fully hadronic mode suffers from large QCD backgrounds, including the production of $t \bar{t}$ events through gluon fusion and also large fake rates for purely light jet processes.

Recent work based on the examination of the jet substructure from highly boosted top quarks have found a tagging efficiency above $40 \%$ for top quark events that satisfy $p_{T}>600 \mathrm{GeV}$, and a mistag rate of about $4 \%[96]$. Other studies have suggested a 35-45\% efficiency may be possible for a mistag rate of around 1\% [97] for highly boosted top jets.

The irreducible $t \bar{t}$ backgrounds cannot be avoided. However, purely QCD mistagged events (such as dijet) can be reduced by using the semi-leptonic mode and assuming a sort of collinear approximation in order to reconstruct the invariant mass of the event given the large missing transverse energy. This requires that the energy of the top quark is large with respect to its mass. However, this method is not the focus of this thesis.

In the study reported in this thesis, I assume a tagging efficiency of $\epsilon_{t}=40 \%$ for 
each top quark in the fully hadronic final state with $\epsilon_{j, \text { mistag }}=1 \%$, as these are the values that were published at the time of the original study [2]. Similar to the bottom quark signal, QCD production of $t \bar{t}$ events are the primary irreducible backgrounds, with dijets as the primary reducible background.

\subsubsection{Tau Leptons}

There are three possible decay modes to consider for pair production of tau leptons: fully leptonic (where $\tau^{+} \tau^{-} \rightarrow \nu_{\tau} \bar{\nu}_{\tau} \ell^{+} \ell^{-} \nu \bar{\nu}$ ), with a branching ratio of $12.4 \%$; semileptonic (where $\tau^{+} \tau^{-} \rightarrow \nu_{\tau} \bar{\nu}_{\tau} \ell \nu+j e t s$ ), with a branching ratio of $45.6 \%$; and purely hadronic events $\left(\tau^{+} \tau^{-} \rightarrow \nu_{\tau} \bar{\nu}_{\tau}+\right.$ jets $)$, with a branching ratio of $42 \%$.

Of hadronic $\tau$ decays, $\approx 77 \%$ have one charged pion track (one-prong), with a tagging efficiency of $50 \%$ and a mistag rate of $0.1 \%$, and $\approx 23 \%$ have three charged pion tracks (three-prong), with a tagging efficiency of $20 \%$ and a mistag rate of $0.1 \%$, both for events with a $E_{T}>100 \mathrm{GeV}[93]$.

Due to the low rest mass of the tau lepton, the collinear approximation is valid for $\tau^{+} \tau^{-}$events, and is assumed to be applied to reconstruct the invariant mass of the $\tau^{+} \tau^{-}$system. This is true even for semileptonic decays, for which the dijet background is negligible. Thus, in practical situations, the semileptonic decay mode may be preferable. The primary background for this mode is $W^{+} W^{-}$, though a large portion of events can be reduced since the on-shell, hadronic $W$ can be vetoed against by reconstructing the larger invariant mass of the $W \rightarrow j j$ decay, and by examining $\Delta \phi$ between the lepton and jets. However, the only fake backgrounds considered here are dijets, as the fully hadronic final state is analyzed. 


\subsubsection{Tagging Summary}

Since the $Z^{\prime}$ decays to pairs of fermions, I have applied an overall fermion efficiency that accounts for branching ratios as well as tagging for both final states. These values are listed in Table 4.1 .

Table 4.1: Summary of the overall efficiencies used for estimating the number of events observed of the given fermion species. The overall efficiency is the tagging efficiency for the observed fermion decay mode times the BR to that final state, squared (one factor for each fermion).

\begin{tabular}{l|c|c} 
Channel & Overall $\epsilon_{f}$ & Jet Mistag $\epsilon_{j}$ \\
\hline$Z^{\prime} \rightarrow \mu^{+} \mu^{-}$ & 0.92 & $\mathrm{n} / \mathrm{a}$ \\
$Z^{\prime} \rightarrow \tau^{+} \tau^{-}$ & 0.08 & $2.0 \times 10^{-6}$ \\
$Z^{\prime} \rightarrow b \bar{b}$ & 0.36 & $2.5 \times 10^{-5}$ \\
$Z^{\prime} \rightarrow t \bar{t}$ & 0.075 & $1.0 \times 10^{-4}$
\end{tabular}

\subsection{Kinematic Cuts}

The values for the fake rates and efficiencies assume the ideal case of perfect alignment within the inner detector and no pile-up. Since tracking is only available within $|\eta|<2.5$, events in which one or both fermion tracks lie outside this region are excluded. In general, a cut of $p_{T}>20 \mathrm{GeV}$ is necessary for flavour tagging. For hadronic decays of $\tau, b$ and $t$ fermions, a stronger $p_{T}$ cut, $p_{T}>0.3 M_{Z^{\prime}}$, is instead used on the reconstructed momentum of the hadronic jets to effectively reduce both the irreducible and dijet backgrounds as compared to the signal. Since the events from the decay of a $Z^{\prime}$ tend towards a harder $p_{T}$ distribution than the QCD backgrounds, a larger percentage of $Z^{\prime}$ events pass this cut than the QCD backgrounds [60]. In order for the phase space contributions to cancel out in the ratio observables, these 
cuts are also applied to the muon final states for the ratio observable calculations (but not for the $\tilde{A}_{C E}$ and $\tilde{A}_{F B}$ ).

Figure 4.4 (a) shows the invariant mass distribution of a $Z^{\prime}$ decaying to $b \bar{b}$ for several models, the QCD $b \bar{b}$ backgrounds, and the light dijet backgrounds without cuts or tagging. Figure 4.4 (b) shows those events that pass the strong $p_{T}$ cut, but without tagging. Fig. 4.4 (c) shows the reduction of the light dijet background after taking into account detector efficiencies and fake rates. Figure 4.4 (c) clearly shows that the application of appropriate flavour tagging algorithms reduces the light dijet backgrounds to a level where a meaningful measurement should be possible.

Only events within an appropriate invariant mass window around the resonance mass are included to improve the signal to background ratio. Events within $\left|M_{Z^{\prime}}-M_{f \bar{f}}\right|<2.5 \Gamma_{Z^{\prime}}$ are included when calculating the total signal. This restricts the background events to the kinematic region directly under the resonance peak. However, this introduces an additional experimental uncertainty due to detector resolution, which smears out the resonance peak, effectively reducing the number of signal events in the peak. Because the ratios of cross section measurements $(b \bar{b}, t \bar{t}$, and $\tau^{+} \tau^{-}$to $\mu^{+} \mu^{-}$final states), include different energy resolutions for each final state involved, the numerical value of the observables will shift from the ideal ratios of couplings. Thus, it will be important to understand detector resolution to accurately extract the underlying cross sections and coupling dependence. In addition, the reduction of the measured signal due to smearing, as compared to background, will increase the experimental errors.

To properly account for detector resolution requires a realistic, detailed simulation for the specific particle identification algorithms being used. This subject is evolving rapidly, and experimentalists are constantly improving their understanding of the energy calibration of the LHC detectors. To gauge the importance of detector resolution, I used estimates from recent detector studies and included them by applying 

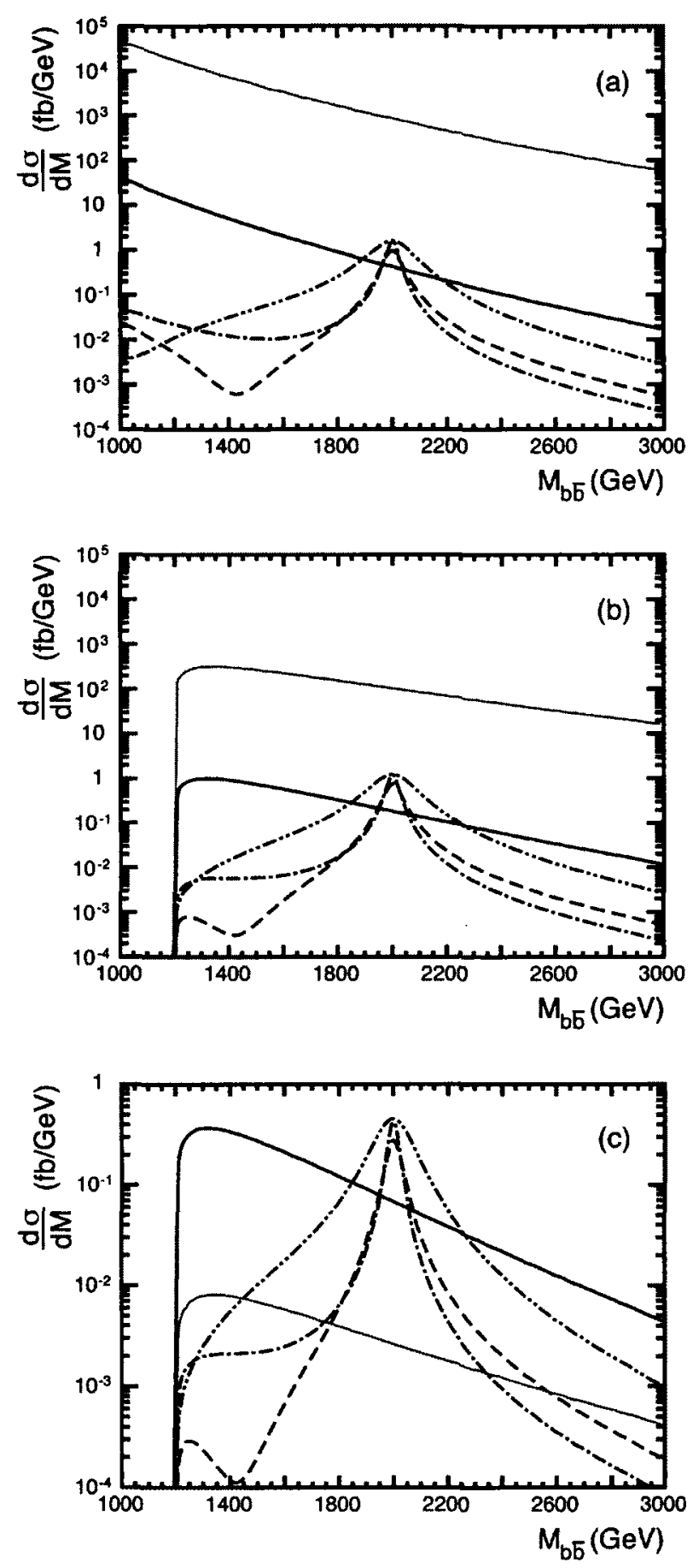

Figure 4.4: $\quad b \bar{b}$ invariant mass distributions for QCD $b \bar{b}$ background (solid, dark), light dijet background (solid, grey), and a $Z^{\prime}$ with $M_{Z^{\prime}}=2.0 \mathrm{TeV}$ for the LRM (dotdash), UUM (dot-dot-dash), and LH (dashed) models. (a) Only includes detector acceptance cuts of $p_{T}>20 \mathrm{GeV}$ and $|\eta|<2.5$. (b) Also includes $p_{T}>0.3 M_{Z^{\prime}}$ cut, as described in the text, but before including tagging efficiencies. (c) Includes the application of cuts, tagging efficiencies, and light jet rejection rates given in Table 4.1. 
a Gaussian smearing to the final state momentum. Studies by the ATLAS collaboration expect between $3 \%$ and $5 \%$ energy resolution for $\mathrm{TeV}$ scale hadronic jets [93] (although the current ATLAS jet calibration gives 5\% resolution [98]). This broadens the resonance, reducing the number of measured signal events within the same invariant mass window. Signal significance may therefore be significantly reduced for narrow resonances where this smearing dominates over the width.

To see how detector resolution affects measurements, the results for the ideal case of no smearing and for a more realistic case of $5 \%$ jet energy resolution for $b$ and $t$ final states and $3 \%$ for muon final states are compared. For the $b \bar{b}$ case I found that for the chosen mass window, as expected, detector resolution has the greatest effect for the narrowest resonances (e.g. the $Z_{\psi}^{\prime}$ ) with virtually no effect on the broadest states (e.g. the $Z_{U U M}^{\prime}$ ). This is shown in Fig. 4.5, where the ratio of the measured to ideal cross sections is plotted versus the width of the $Z^{\prime}$ for several values of energy resolution. The situation for the $t \bar{t}$ final state is less conclusive. A recent ATLAS study [99] gives a resolution of $4.6 \%$ for the $t \bar{t}$ invariant mass from a narrow resonance. However, another ATLAS study [100] gives a resolution of $9-10 \%$ for a $1 \mathrm{TeV} Z^{\prime}$ with width $\Gamma_{Z^{\prime}} / M_{Z^{\prime}}=3.3 \%$. The range of these expectations demonstrates the difficulty in trying to predict the effects of detector resolution for these measurements.

Using the same procedure used to gauge the importance of detector resolution for the $b \bar{b}$ channel, as in Fig. 4.5, I found that a usable signal can be measured in the both the $b \bar{b}$ and $t \bar{t}$ channels for all models considered in this paper except for possibly $Z_{\eta}$ and $Z_{\psi}$. This is consistent with the findings of Barger, Han and Walker [101].

Reconstruction of $\tau^{+} \tau^{-}$final states are complicated due to the missing energy from the neutrinos in the $\tau$ decays. LHC studies of $\tau^{+} \tau^{-}$final states with respect to Higgs searches have found a resolution on the reconstructed Higgs mass of $M_{H} \sim 10 \%$ $[99,102,103]$. This mass resolution is not included in the results.

Thus, an estimate is provided for the effects that detector energy resolution will 


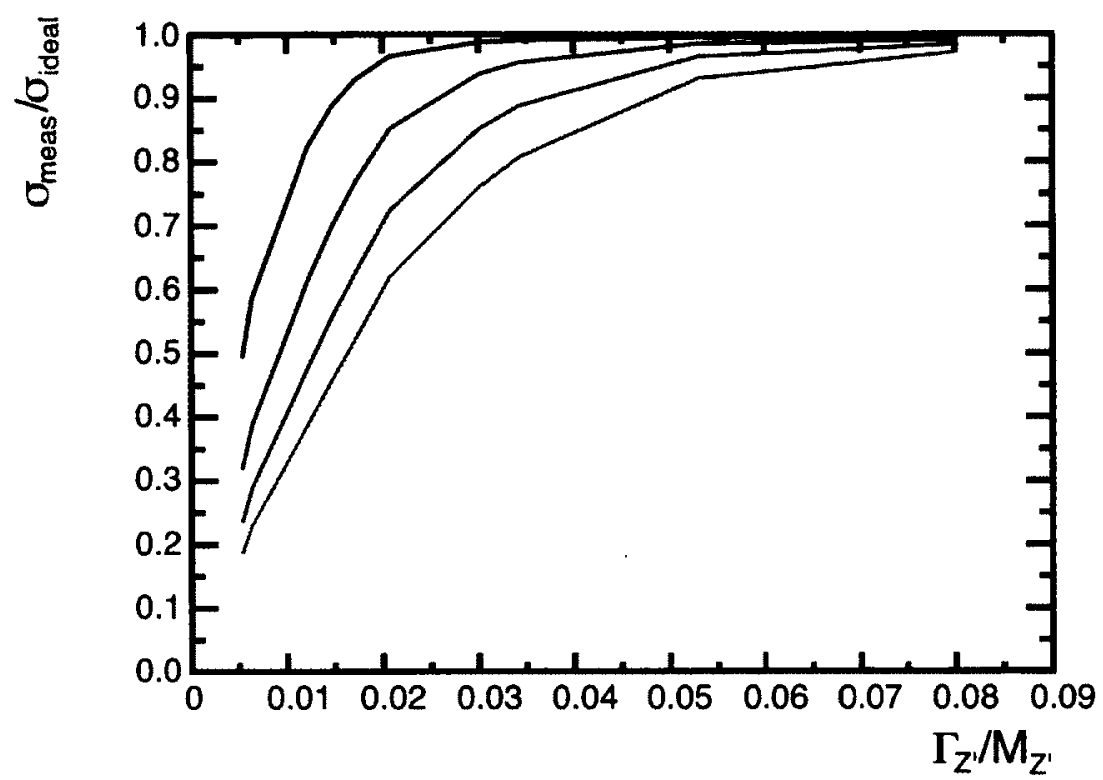

Figure 4.5: Fraction of events remaining after including energy resolution smearing for a fixed invariant mass window based on the $Z^{\prime}$ width. From top to bottom, lines correspond to $3 \%, 5 \%, 7 \%$ and $9 \%$ energy resolution. Data is taken from model based simulations with actual decay widths of the $Z^{\prime}$ from the model, given as a fraction of the $Z^{\prime}$ mass.

have on the precision of the measurements, past experience shows that experimentalists eventually exceed initial expectations. In addition to this, the reduced signal to background caused by detector resolution can be mitigated to some extent by higher luminosities and better identification efficiencies, which would improve overall statistics. 


\section{Chapter 5}

\section{Results}

\subsection{Centre-Edge Asymmetry}

The usefulness of using the $\Delta \eta$ distribution, and correspondingly defined centre-edge asymmetry, to distinguish the spin of the resonance is clear, as shown in Fig. 5.1. This form of the centre-edge asymmetry is as distinctively capable of distinguishing the spin of a resonance as one based on the angular distribution, but trivializes the calculation and reduces any contribution from energy scale uncertainty to the calculation.

Many of the resonances exhibit narrow widths, such that the observed width may be dominated by smearing effects due to energy resolution effects in the detectors. Events within one dilepton invariant mass bin, as defined by the ATLAS TDR [104] are used for the centre-edge asymmetry measurement, which corresponds to $\Delta M=42.9 \mathrm{GeV}$ for a $1.5 \mathrm{TeV}$ resonance, as in Ref. [105]. Increasing the size of the invariant mass window for broader peaks would result in a larger number of events passing selection cuts, thus would reduce the statistical uncertainty, but may increase backgrounds.

Table 5.1 shows the expected centre-edge asymmetry for spin- 0 , spin- 1 and spin- 2 resonances, analogous to the study performed by Dvergsnes, et al. [106], assuming muon final states with $96 \%$ detection efficiency [93]. From Table 5.1 it is clear that 


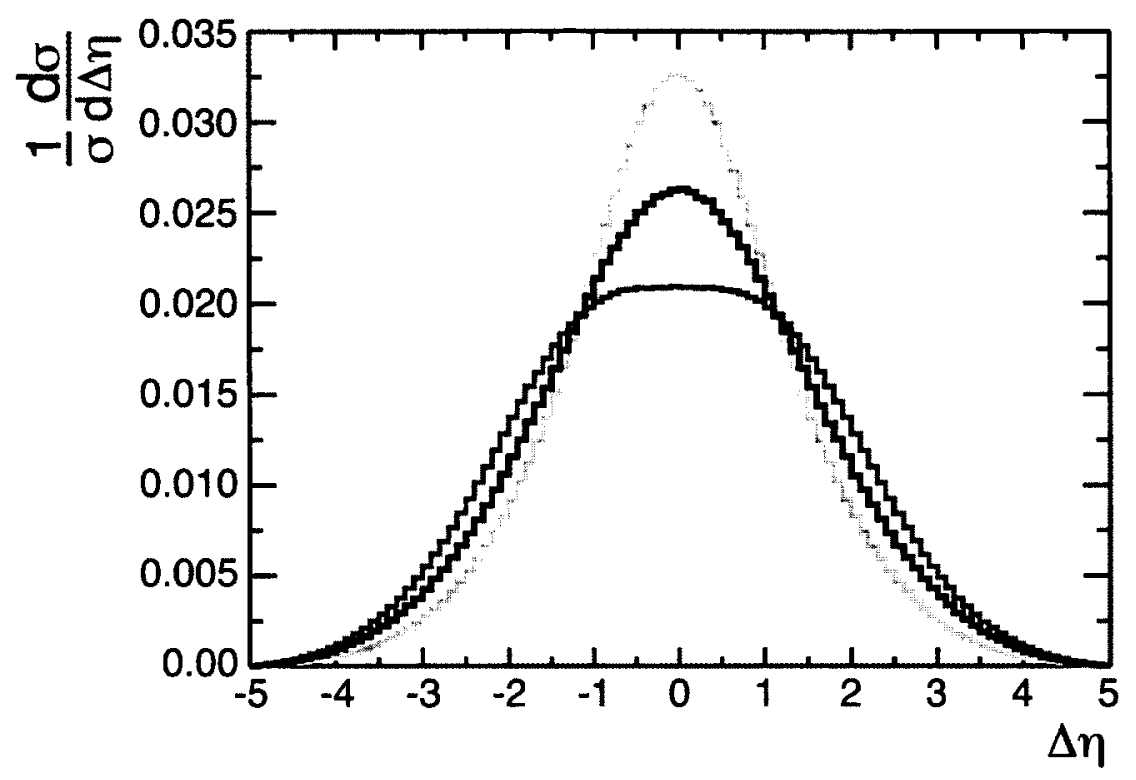

Figure 5.1: Normalized $\Delta \eta$ distribution including detector acceptance cuts $\left(\left|\eta_{l}\right|<\right.$ $\left.2.5, p_{T_{l}}>20 \mathrm{GeV}\right)$ and only including events within $\left|M_{R}-M_{l^{+} l^{-}}\right|<0.5 \Delta M$. These cuts reduce the number of measurable events with large values of $|\Delta \eta| . R=\tilde{\nu}$ (black), $Z^{\prime}$ (dark grey/orange), $G$ (light grey/yellow), where only one spin-1 distribution is shown due to the model independent nature of the spin-1 measurement.

if a $Z^{\prime}$ were observed, a $G$ or $\tilde{\nu}$ could be ruled out. Likewise, an $A_{C E}$ measurement would strongly discriminate against the $Z^{\prime}$ or $\tilde{\nu}$ hypothesis if a $G$ were observed.

\subsection{Forward-Backward Asymmetry}

\section{Lepton Final States}

Figure 5.2 shows the normalized distribution of forward minus backward tagged events, dependent on the rapidity of the $Z^{\prime}$, using the $\Delta|\eta|$ tagging method. As

in the conventional method for finding the $\tilde{A}_{F B}$, forward minus backward tagged events cancel out in the central region of $y_{Z^{\prime}}$ due to the equal probability of either 
Table 5.1: $\tilde{A}_{C E}$ values with corresponding statistical uncertainties for $100 \mathrm{fb}^{-1}$ integrated luminosity, $p_{T_{l}}>20 \mathrm{GeV},\left|\eta_{l}\right|<2.5$, within one bin $\Delta M_{l^{+} l^{-}}=42.9 \mathrm{GeV}$ and $M_{R}=1.5 \mathrm{TeV}$. Also shown are the expected number of total events for each model assuming $100 \mathrm{fb}^{-1}$ integrated luminosity.

\begin{tabular}{|lcc|}
\hline Model & $\tilde{A}_{C E} \pm \delta \tilde{A}_{C E}$ & N Events \\
\hline$E_{6} \chi$ & $-0.106 \pm 0.017$ & 3875 \\
$E_{6} \psi$ & $-0.095 \pm 0.022$ & 2223 \\
$E_{6} \eta$ & $-0.092 \pm 0.021$ & 2480 \\
LR Symmetric & $-0.099 \pm 0.018$ & 3350 \\
Sequential SM & $-0.097 \pm 0.016$ & 4162 \\
Littlest Higgs & $-0.095 \pm 0.001$ & 6217 \\
Simplest Little Higgs & $-0.094 \pm 0.017$ & 3542 \\
RS Graviton & $+0.228 \pm 0.011$ & 8208 \\
R-parity violating $\tilde{\nu}$ & $+0.055 \pm 0.066$ & 251 \\
\hline
\end{tabular}

proton contributing the interacting quark. In this region, there is a higher probability of incorrectly assigning the forward or backward direction, which gives a small "wrong" contribution to the $\tilde{A}_{F B}$ measurement, as discussed in 4.2. For this reason, it has been suggested that the central region, $\left|y_{Z^{\prime}}\right|<y_{\min }$, be excluded in the measurement [107]. This effect is statistically insignificant, however, and the coupling dependency of the forward-backward asymmetry can still be determined without this constraint on $\left|y_{Z^{\prime}}\right|[47]$.

Another consideration in the issue of excluding the central region is that the number of events that remain after subtracting $(F-B)$ in this region is small, as evident from Fig. 5.2, while the total number of events in this region $(F+B)$ is large. Excluding the events in the central region would increase the magnitude of $\tilde{A}_{F B}$, by reducing the number of events included in the denominator, potentially making models more numerically distinguishable. However, it can be shown that increasing $y_{\min }$ results in 


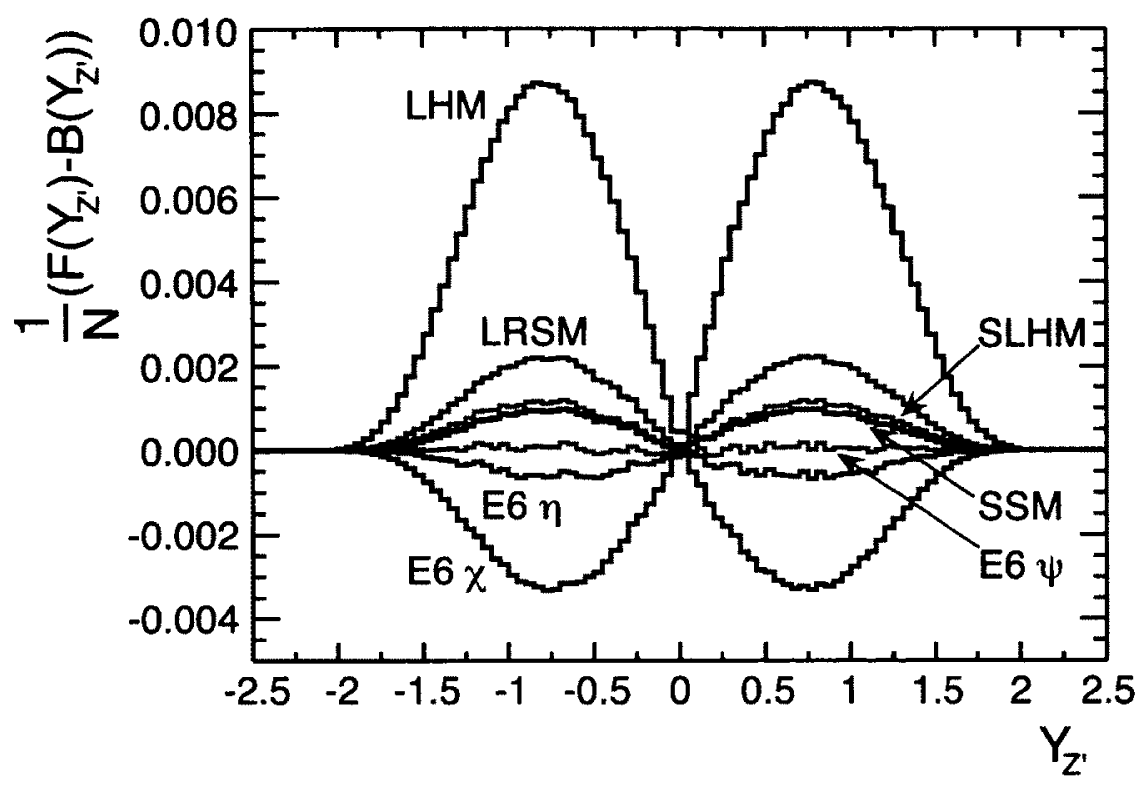

Figure 5.2: $\quad \tilde{A}_{F B}$ as a function of the $Z^{\prime}$ rapidity using $\Delta|\eta|$ to distinguish forward from backward tagged events. From top to bottom, the models are LHM, LRSM, SLHM, SSM, $E_{6} \psi, E_{6} \eta, E_{6} \chi$. The $Z^{\prime}$ rapidity is defined as $Y_{Z^{\prime}}=\frac{1}{2} \ln \frac{E+p_{z}}{E-p_{z}}$.

an increase in the relative statistical uncertainty. The percentage statistical uncertainty is proportional to $1 / \sqrt{N}$, which decreases as total events increase. I therefore conclude that little is gained by excluding events with small $y_{Z^{\prime}}$, and suggest that the whole rapidity region be included to decrease relative statistical uncertainty and further simplify the $\tilde{A}_{F B}$ measurement.

Using this method, the $\tilde{A}_{F B}$ was calculated for the $E_{6}$ models $(\psi, \chi, \eta)[54]$, the Left Right Symmetric model [62], the Littlest Higgs model [32], the Simplest Little Higgs model [66], and the Sequential Standard model. The on-peak versus off-peak $\tilde{A}_{F B}$ values are plotted in Fig. 5.3, where on-peak includes events which satisfy $\mid M_{l^{+} l^{-}}-$ $M_{Z^{\prime}} \mid<3 \Gamma_{Z^{\prime}}$ and off-peak includes events which satisfy $2 / 3 M_{Z^{\prime}}<M_{l^{+} l^{-}}<M_{Z^{\prime}}-3 \Gamma_{Z^{\prime}}$, similar to the cuts used by Petriello and Quackenbush [59].

It might also be possible to include some events in the forward regions of the 


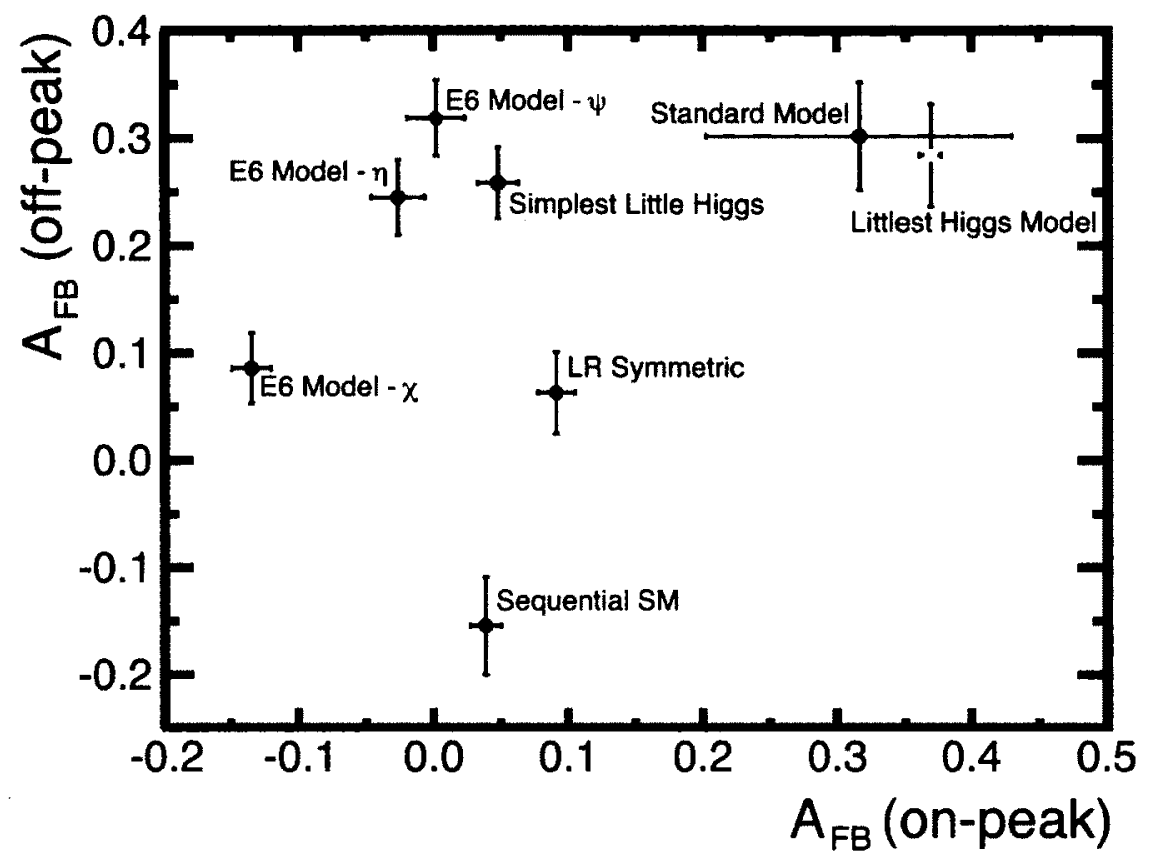

Figure 5.3: $\quad \tilde{A}_{F B}$ off-peak versus on-peak for a variety of models, including detector acceptance limits and kinematic cuts as previously listed. Standard Model measurement determined from the standard model Drell-Yan cross section, with onpeak events within $\left|M_{l^{+} l^{-}}-M_{Z^{\prime}}\right|<100 \mathrm{GeV}$ and off-peak events within $2 / 3 M_{Z^{\prime}}<$ $M_{l^{+} l^{-}}<M_{Z^{\prime}}-300 \mathrm{GeV}$ to include sufficient statistics, at $100 \mathrm{fb}^{-1}$.

calorimeter (FCAL) using this technique. While a muon signature appears as missing $E_{T}$ in the FCAL, it may be possible to distinguish an electron from a jet in the FCAL due to the differences in the showering. The signal would require triggering off of a single, high $p_{T}$ electron in the $|\eta|<2.5$ region, with an electron-jet in the FCAL. Determining the charge sign of the single electron in the central regions would distinguish whether this is a "forward" or "backward" tagged event. It is not clear what the signal efficiency of this method is, as reducible backgrounds include $W+j$ and others that might have low rejection rates. Extending the rapidity range has the potential of increasing the statistics and remains an interesting possibility for further study. 


\section{Hadronic Final States}

As in all measurements involving third generation fermions, the challenge is extracting the events of interest from a large standard model background and accumulating sufficient statistics to make a meaningful measurement. The QCD backgrounds (heavy quark production and light jet mistags) for these measurements are forward-backward symmetric at tree level, which should allow a heavy quark $\tilde{A}_{F B}$ to be sensitive to the presence of a $Z^{\prime}$, or other new physics. Rather than subtracting out the backgrounds, the totals of signal plus backgrounds is included in the forward and backward regions. Since the backgrounds are symmetric, they do not contribute to the numerator of Eq. 4.16, but do for the denominator. In a practical scenario, it would be impossible to determine a priori which events arise from the $Z^{\prime}$ signal versus the background, and so this more reasonably simulates a measurement at the LHC.

Figure 5.4 shows the expected results for the $\tilde{A}_{F B}$ in the $b \bar{b}$ and $t \bar{t}$ channels for several models assuming $M_{Z^{\prime}}=1.5 \mathrm{TeV}, L=100 \mathrm{fb}^{-1}$ for $14 \mathrm{TeV}$ collisions, and the kinematic cuts described in Sec. 4.4, not including detector resolution effects. While large uncertainties are apparent for some models, a reasonable measurement can still be expected for many models that have universal couplings, including the Left-Right Symmetric Model, the various Little Higgs models, and models with nonuniversal couplings. Table 5.2 gives the corresponding numerical values for $\tilde{A}_{F B}^{t \bar{t}, b \bar{b}}$ and the statistical uncertainties that can be expected for $100 \mathrm{fb}^{-1}$ integrated luminosity.

To estimate the effects of detector resolution on the $\tilde{A}_{F B}$ measurement, Fig. 5.5 shows the same measurement but includes the detector resolution as described earlier. The broadening of the peak from smearing results in larger statistical uncertainties due to the larger number of background events included. Figure 5.5 assumes an integrated luminosity of $300 \mathrm{fb}^{-1}$ to improve the statistical uncertainty. Taking into account detector resolution both reduces the statistics and shifts the observed values 


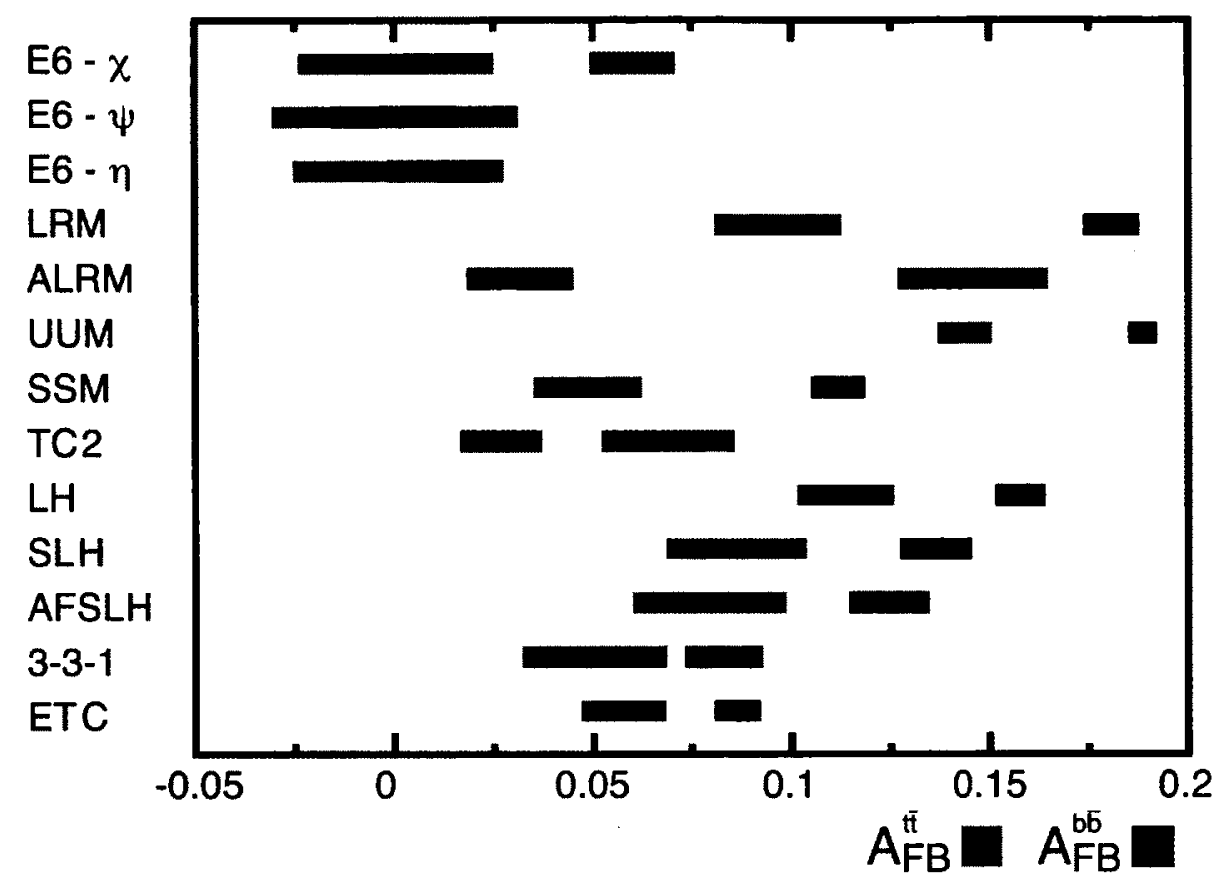

Figure 5.4: $\tilde{A}_{F B}^{b \bar{b}}$ (black bars) and $\tilde{A}_{F B}^{t \bar{t}}$ (grey bars) for a $Z^{\prime}$ with a mass of $1.5 \mathrm{TeV}$. Statistical errors include contributions from QCD backgrounds and light dijets assuming $100 \mathrm{fb}^{-1}$ luminosity.

for the $\tilde{A}_{F B}$ measurements. While the $\tilde{A}_{F B}$ still has some resolving power with $b \bar{b}$ final states it is not clear how useful the $t \bar{t}$ final states will be, due to low statistics arising from branching ratios and tagging issues. It is possible that if some effort were made to disentangle detector resolution from the underlying cross section these results could be improved. Nevertheless, I expect that $\tilde{A}_{F B}$ measurements would still be useful to constrain the $Z^{\prime}$-fermion couplings as part of a global fit. 




Figure 5.5: $\tilde{A}_{F B}^{b \bar{b}}$ (black bars) and $\tilde{A}_{F B}^{t \bar{t}}$ (grey bars) including detector resolution as described in the text. Statistical errors include contributions from QCD backgrounds and light dijets assuming $300 \mathrm{fb}^{-1}$ luminosity. 
Table 5.2: $\quad \tilde{A}_{F B}$ values for $b$ - and $t$-quark final states with corresponding statistical uncertainties, assuming $M_{Z^{\prime}}=1.5 \mathrm{TeV}$ and $L=100 \mathrm{fb}^{-1}$. Cuts include $p_{T}>$ $0.3 M_{Z^{\prime}} \mathrm{GeV},\left|\eta_{t, b}\right|<2.5$, within $\left|\Delta M_{q \bar{q}}-M_{Z^{\prime}}\right|<2.5 \Gamma_{Z^{\prime}}$. The first set of data, labelled as "ideal detector", do not include detector resolution smearing for the final state fermions, while the second set of data does include the effects of detector resolution.

\begin{tabular}{|l|cc|cc|}
\hline Model & $\tilde{A}_{F B}^{t} \pm \delta \tilde{A}_{F B}^{t}$ & $\tilde{A}_{F B}^{b} \pm \delta \tilde{A}_{F B}^{b}$ & $\tilde{A}_{F B}^{t} \pm \delta \tilde{A}_{F B}^{t}$ & $\tilde{A}_{F B}^{b} \pm \delta \tilde{A}_{F B}^{b}$ \\
\hline & \multicolumn{2}{|c|}{ ideal detector } & \multicolumn{2}{|c|}{ including detector resolution } \\
\hline$E_{6} \chi$ & $0.00 \pm 0.02$ & $0.060 \pm 0.011$ & $0.00 \pm 0.03$ & $0.017 \pm 0.016$ \\
$E_{6} \psi$ & $0.00 \pm 0.03$ & $0.000 \pm 0.016$ & $0.00 \pm 0.05$ & $0.00 \pm 0.02$ \\
$E_{6} \eta$ & $0.00 \pm 0.03$ & $0.013 \pm 0.014$ & $0.00 \pm 0.04$ & $0.00 \pm 0.02$ \\
LRM & $0.097 \pm 0.016$ & $0.181 \pm 0.007$ & $0.04 \pm 0.02$ & $0.075 \pm 0.011$ \\
ALRM & $0.146 \pm 0.019$ & $0.032 \pm 0.013$ & $0.04 \pm 0.03$ & $0.006 \pm 0.016$ \\
UUM & $0.144 \pm 0.007$ & $0.189 \pm 0.004$ & $0.073 \pm 0.011$ & $0.094 \pm 0.006$ \\
SSM & $0.049 \pm 0.014$ & $0.112 \pm 0.007$ & $0.02 \pm 0.02$ & $0.044 \pm 0.010$ \\
TC2 & $0.069 \pm 0.017$ & $0.027 \pm 0.010$ & $0.02 \pm 0.02$ & $0.01 \pm 0.01$ \\
LH & $0.114 \pm 0.012$ & $0.158 \pm 0.006$ & $0.047 \pm 0.018$ & $0.063 \pm 0.009$ \\
SLH & $0.086 \pm 0.018$ & $0.136 \pm 0.009$ & $0.03 \pm 0.03$ & $0.042 \pm 0.014$ \\
AFSLH & $0.079 \pm 0.019$ & $0.124 \pm 0.010$ & $0.02 \pm 0.03$ & $0.035 \pm 0.015$ \\
$3-3-1$ & $0.050 \pm 0.018$ & $0.083 \pm 0.010$ & $0.02 \pm 0.03$ & $0.023 \pm 0.014$ \\
ETC & $0.058 \pm 0.011$ & $0.086 \pm 0.006$ & $0.022 \pm 0.015$ & $0.031 \pm 0.008$ \\
\hline
\end{tabular}




\subsection{Third Generation Ratios}

\subsubsection{Model Discrimination using $t \bar{t}$ and $b \bar{b}$ to $\mu^{+} \mu^{-}$Produc- tion Ratios}

The results shown in this subsection include the assumption that a $Z^{\prime}$ has been discovered and its mass and width measured [51,108-110] such that the appropriate $M_{Q \bar{Q}}$ and $p_{T}$ cuts described in Sec. 4.4 can be applied. Results include statistical uncertainties for signal plus background, including mistagged light jet events, for efficiencies $\epsilon_{\mu^{+} \mu^{-}}, \epsilon_{b \bar{b}}$, and $\epsilon_{t \bar{t}}$ from Table 4.1. These observables assume that the event excess from the $Z^{\prime}$ signal can be extracted from the background events in order to relate the ratio of cross sections to the $Z^{\prime}$ couplings.

The results for $R_{b / \mu}$ and $R_{t / \mu}$ are shown in Figures 5.6 and 5.7, where Fig. 5.7 expands the view to show the distinctive measurements that would be observed for models with non-universal couplings. The error bars include the $1 \sigma$ statistical uncertainties based on events for an integrated luminosity of $L=100 \mathrm{fb}^{-1}$. It is clear that the measurements for models with non-universal couplings and mixing parameters that result in large couplings for third generation quarks are quite distinctive, making this a possible hallmark measurement for these models.

Figures 5.8 and 5.9 display the results for $R_{b / \mu}$ and $R_{t / \mu}$ including a detector resolution of $5 \%$ for $b$ and $t$ final states, and 3\% for muons. Comparing Fig. 5.9 with Fig. 5.7, it is clear that the effect of resolution is small for broad models such as ETC, TC2 and UUM. On the other hand, the effect is much more significant for the narrow models such as the E6, SLH, and AFSLH models, affecting both the ratio value and the expected statistical uncertainty. There are two approaches to account for resolution effects in these measurements. The experimentalists can try to disentangle resolution effects from the underlying cross section to give the "theoretical" cross 




Figure 5.6: Measurements of the ratio of $t \bar{t}$ and $b \bar{b}$ to $\mu^{+} \mu^{-}$cross sections within $2.5 \Gamma$ of $M_{Z^{\prime}}$, for models with universal couplings. Black bars correspond to expected $1 \sigma$ statistical uncertainties for $M_{Z^{\prime}}=1.5 \mathrm{TeV}$ and grey bars to $M_{Z^{\prime}}=2.5 \mathrm{TeV}$.

section or they can compare to Monte Carlo simulations that account for detector resolution. In any case, the sensitivity of measurements for narrow models to detector resolution will lead to systematic uncertainties in the measurements that need to be taken into account.

For some models, including models with non-universal couplings (i.e. the $E_{6}$ model and the LR Symmetric models), the predictions for $R_{b / \mu}$ and $R_{t / \mu}$ are dependent on the mixing angle between subgroups in the model. This is illustrated in Fig. 5.10 which plots the ideal $R_{t / \mu}-R_{b / \mu}$ ratio values while varying the mixing parameters. 


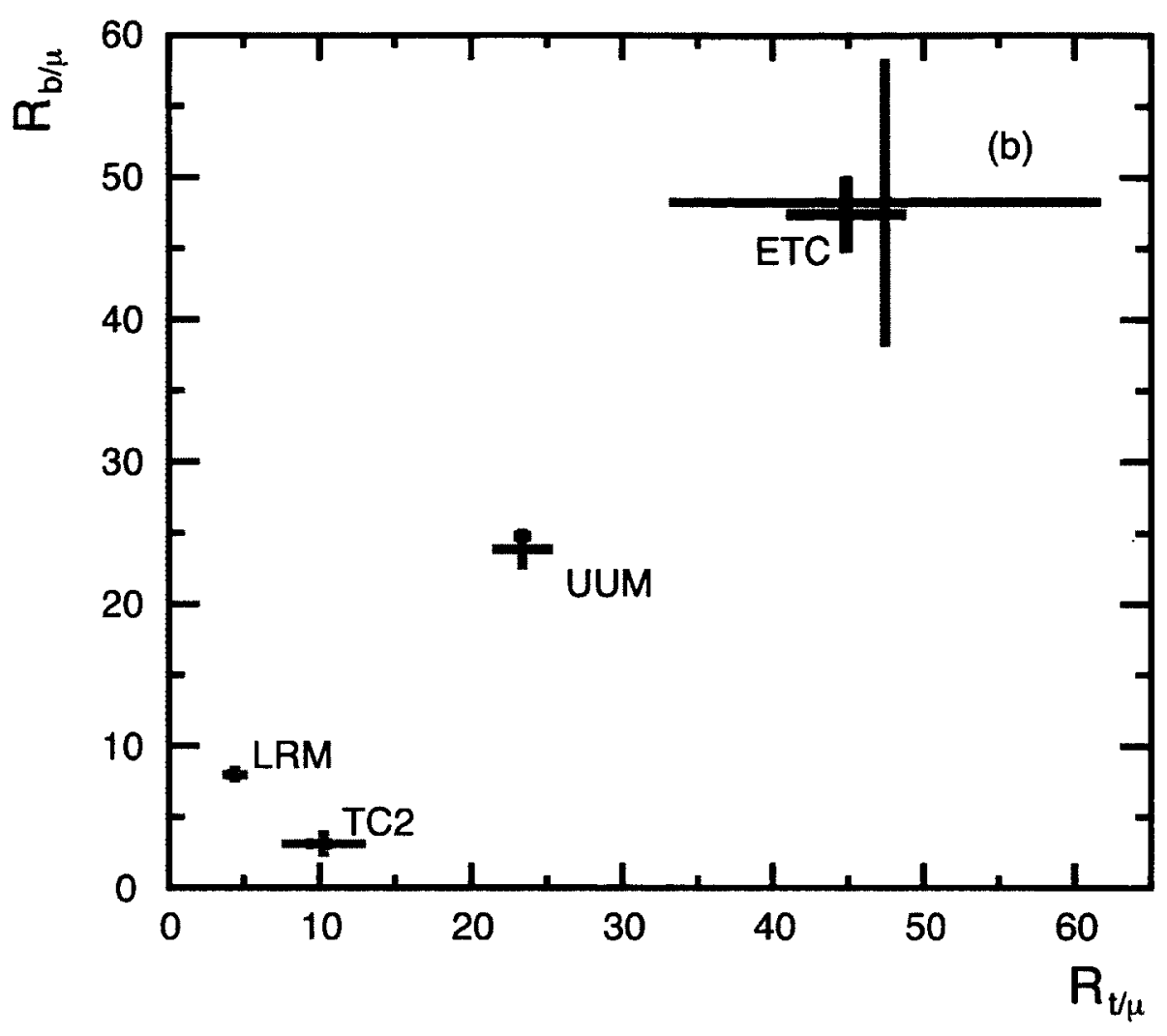

Figure 5.7: Measurements of the ratio of $t \bar{t}$ and $b \bar{b}$ to $\mu^{+} \mu^{-}$cross sections within $2.5 \Gamma$ of $M_{Z^{\prime}}$, for models with non-universal couplings. Black bars correspond to expected $1 \sigma$ statistical uncertainties for $M_{Z^{\prime}}=1.5 \mathrm{TeV}$ and grey bars to $M_{Z^{\prime}}=$ $2.5 \mathrm{TeV}$.

These ratios were determined for a $Z^{\prime}$ mass of $1.5 \mathrm{TeV}$, although only the uncertainties are sensitive to $M_{Z^{\prime}}$ - the observable values are stable over the viable $Z^{\prime}$ mass range. The following ranges for mixing angles were used: for the LR Symmetric model, both with standard and alternate isospin assignments, the mixing parameter is constrained by $0.55 \leq\left(g_{R} / g_{L}\right) \leq 1[55]$; for the UUM, $\phi$ is constrained by $0.22 \leq \sin \phi \leq 0.99[111]$; and for the remaining models no specific limits could be found in the literature that were not directly tied to the mass of the $Z^{\prime}$. Some regions of parameter space result in overlap in the $R_{t / \mu}-R_{b / \mu}$ measurements, as shown in Fig. 5.10. Consequently, other measurements will be necessary to distinguish between 


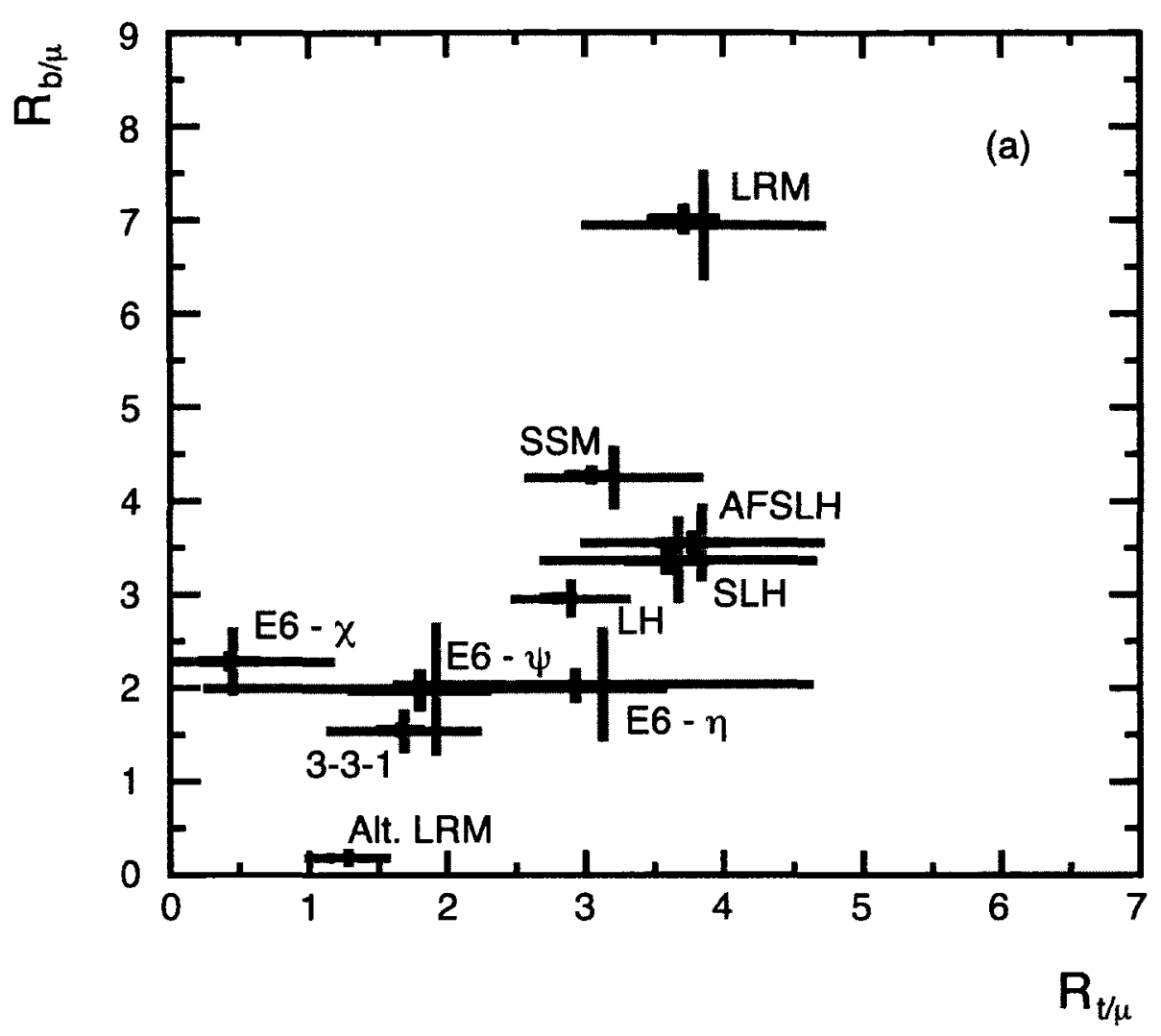

Figure 5.8: Measurements of the ratio of $t \bar{t}$ and $b \bar{b}$ to $\mu^{+} \mu^{-}$cross sections within $2.5 \Gamma$ of $M_{Z^{\prime}}$ including detector resolution of $5 \%$ for $b$ and $t$ final states, and $3 \%$ for muons. Figure includes models with universal couplings, with labelling is as in Fig. 5.6.

models for these values of the model parameters.

It is important to note that the region of overlap in the $R_{t / \mu}-R_{b / \mu}$ plane between models with and without universal couplings occurs for parameter values that are not of particular interest. In the case of the UUM model, the overlap occurs for parameter values where leptons have preferential couplings and in the case of the ETC and TC2 models the overlap occurs when the first two generations of fermions have preferential couplings. Since these models are constructed such that the top quark plays a role in EWSB, one would not expect their mixing angles to take values in the overlap region.

A final observation is that the UUM and ETC models are indistinguishable using 


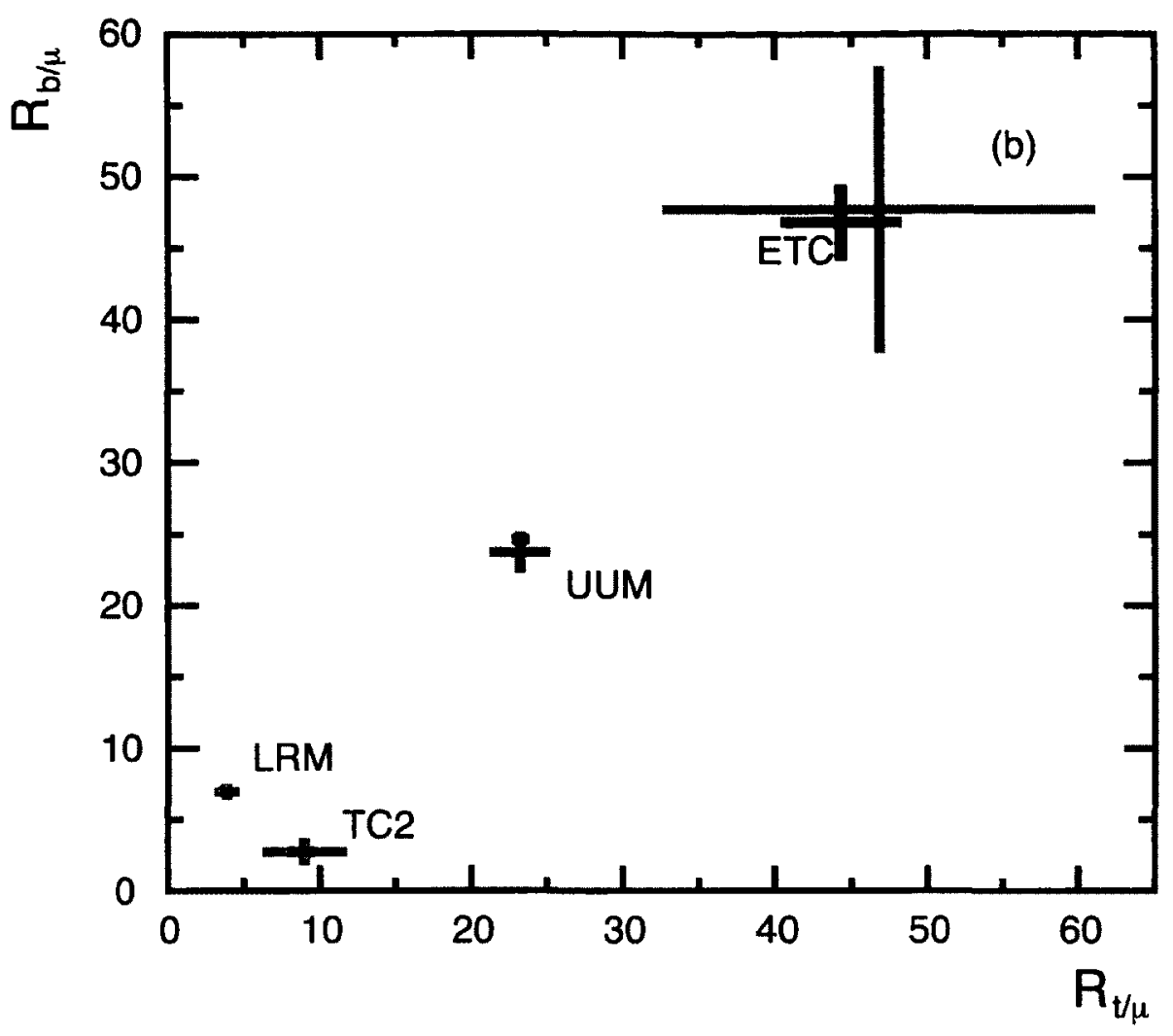

Figure 5.9: Measurements of the ratio of $t \bar{t}$ and $b \bar{b}$ to $\mu^{+} \mu^{-}$cross sections within $2.5 \Gamma$ of $M_{Z^{\prime}}$ including detector resolution of $5 \%$ for $b$ and $t$ final states, and $3 \%$ for muons. Figure includes models with non-universal couplings, with labelling is as in Fig. 5.7.

only measurements of $R_{t / \mu}$ and $R_{b / \mu}$, for any value of mixing parameter. However, in this case, the ratio of tau to muon events at the LHC will discriminate generation dependent couplings. This is examined in the next subsection. 


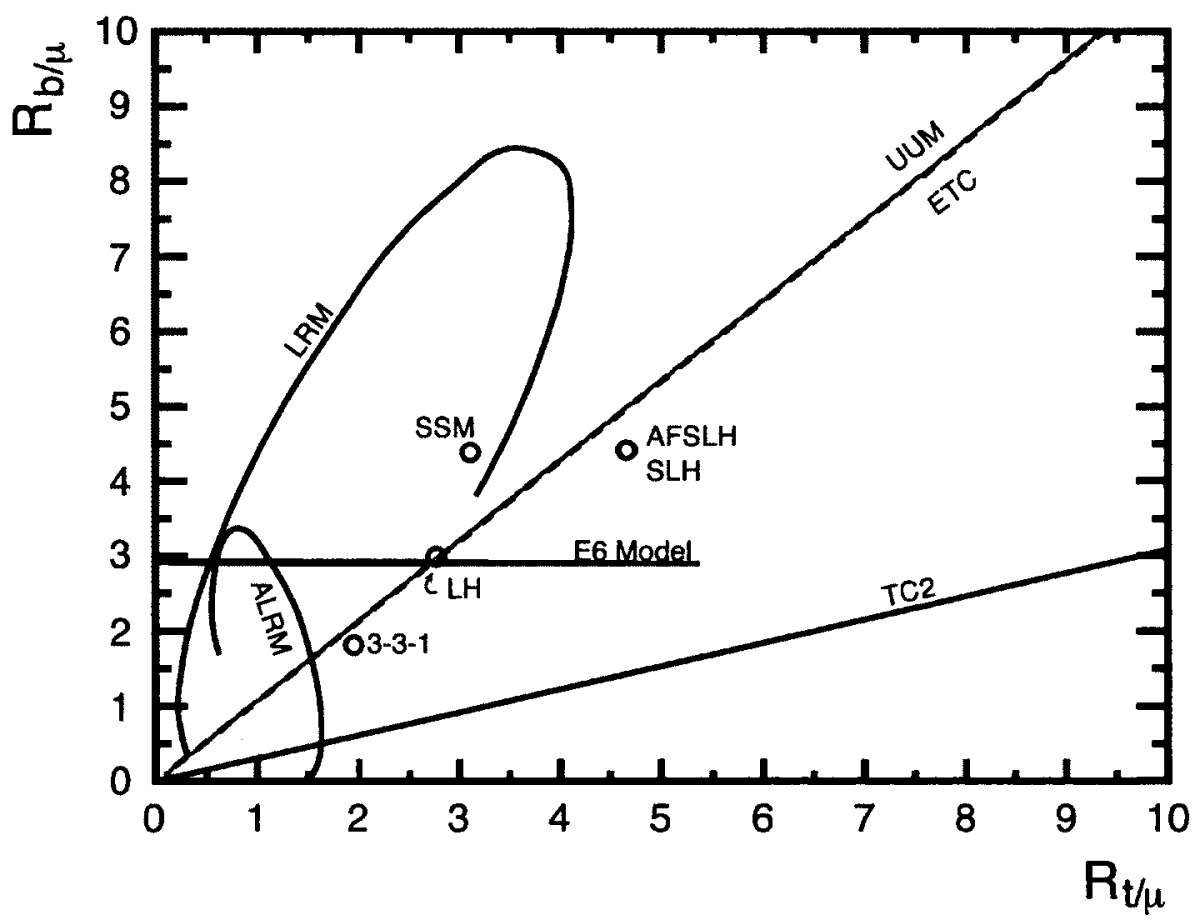

Figure 5.10: The evolution of $R_{b / \mu}$ vs $R_{t / \mu}$, spanning the model parameter range as given in the text, for the models labelled in the figure. 


\subsubsection{Extracting Mixing Parameters Using $R_{\tau / \mu}$}

Measuring generation universality will be an important step in distinguishing or ruling out TC2 and ETC type models, and for distinguishing between $Z^{\prime}$ models such as the ETC and UUM. The simplest and cleanest way to measure the degree of universality is to determine the ratio of $\sigma\left(p p \rightarrow Z^{\prime} \rightarrow \tau^{+} \tau^{-}\right) / \sigma\left(p p \rightarrow Z^{\prime} \rightarrow \mu^{+} \mu^{-}\right)$. For completeness, I also considered, but did not include, a measurement of the ratio of $t$ - to $c$-quark cross sections but such a measurement does not appear promising due to low tagging efficiencies of the charm quark, and indistinguishability of the charm quark from the light jet backgrounds.

The ratio of $R_{\tau / \mu}=\sigma\left(p p \rightarrow Z^{\prime} \rightarrow \tau^{+} \tau^{-}\right) / \sigma\left(p p \rightarrow Z^{\prime} \rightarrow \mu^{+} \mu^{-}\right)$is shown in Fig. 5.11. Only statistical errors are shown for a semi-idealized detector with perfect energy resolution.

It is clear from Fig. 5.11 that models with generation universality (including the Ununified Model) will yield measurements of $R_{\tau / \mu} \simeq 1$ with reasonable precision. In contrast, models with generation dependent couplings show a large, measurable variation from unity. The dependence of $R_{\tau / \mu}$ on the mixing angle between the gauge groups of the theory are given by:

$$
\frac{\Gamma\left(Z_{E T C}^{\prime} \rightarrow \tau^{+} \tau^{-}\right)}{\Gamma\left(Z_{E T C}^{\prime} \rightarrow \mu^{+} \mu^{-}\right)} \propto \tan ^{4} \phi_{E T C}
$$

and

$$
\frac{\Gamma\left(Z_{T C 2}^{\prime} \rightarrow \tau^{+} \tau^{-}\right)}{\Gamma\left(Z_{T C 2}^{\prime} \rightarrow \mu^{+} \mu^{-}\right)} \propto \cot ^{4} \phi_{T C 2}
$$

As pointed out, these results do not include finite detector resolution and in addition, measurements of $\tau^{+} \tau^{-}$final states have the additional complication of missing energy due to neutrinos in the $\tau$ decays. However, the non-universal $Z$ 's have large decay widths, thus interesting measurements of a model with non-universal couplings

should be minimally affected by detector resolution. On the other hand, some of the generation universal $Z^{\prime}$ models are relatively narrow so that the resonances will be 


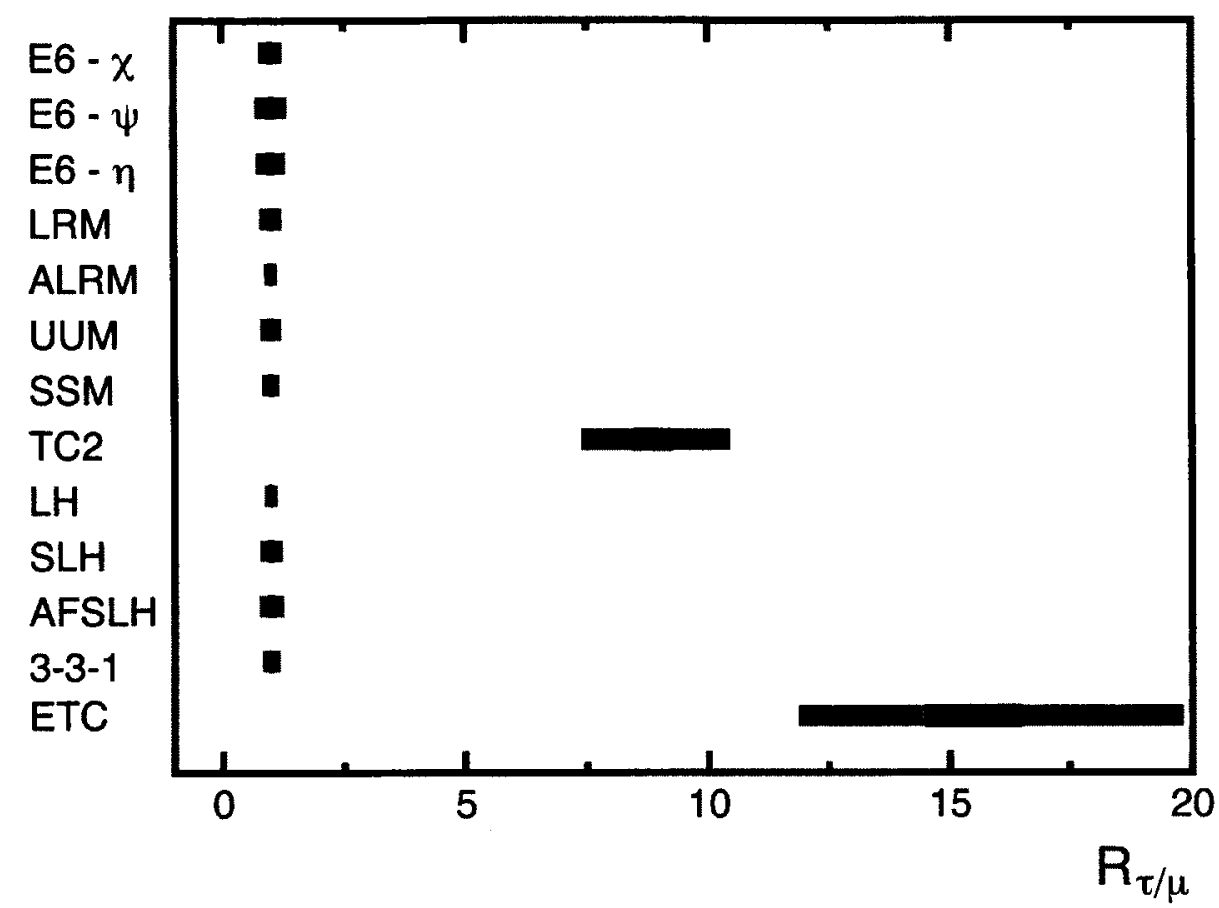

Figure 5.11: $R_{\tau / \mu}$ for models with universal and non-universal fermion couplings. The ratios are obtained for tau and muon events satisfying $\left|M_{f \bar{f}}-M_{Z^{\prime}}\right|<2.5 \Gamma_{Z^{\prime}}$, along with the other cuts described in the text. The dark bars are for $M_{Z^{\prime}}=1.5 \mathrm{TeV}$ and the grey bars for $M_{Z^{\prime}}=2.5 \mathrm{TeV}$ (for $\sin \phi_{T C 2}=0.5$ and $\sin \phi_{E T C}=0.9$ ).

smeared out in the $\tau^{+} \tau^{-}$final state and some care will have to be taken in choosing appropriate invariant mass windows. We note that both ATLAS [112] and CMS [113] have recently measured $\sigma\left(p p \rightarrow Z^{0}\right) \times B r\left(Z^{0} \rightarrow \tau^{+} \tau^{-}\right)$with errors of roughly $20 \%$ and $10 \%$ respectively in the $\tau_{h} \tau_{\ell}$ modes, which bodes well for a measurement of $R_{\tau / \mu}$. With higher luminosity, these measurements should be significantly more accurate.

Given the fundamental nature of the gauge group mixing angle, measuring the value of $\phi$ would be vital input into reconstructing the Lagrangian of the underlying theory. Figure 5.12 shows how well such a measurement can be made for the TC2 and ETC models using $R_{\tau / \mu}$ assuming $M_{Z^{\prime}}=1.5 \mathrm{TeV}$ and $L=100 \mathrm{fb}^{-1}$ for the semi-idealized detector. In these plots the $x$-axis corresponds to the assumed value of the mixing parameter and the $y$-axis corresponds to the measured value of the mixing 
parameter with the spread in the vertical direction corresponding to 1- and 2- $\sigma$ limits of $R_{\tau / \mu}$ for the input parameter value and measured value. The parameter range on the $x$-axis is chosen to correspond to the range where the $Z^{\prime}$ width is less than $10 \%$ of the $Z^{\prime}$ mass. As before, the backgrounds are included in estimating the statistical errors and the same kinematic cuts are imposed, as before.

The limits on the mixing angle found in this analysis could be further constrained by including more observables, such as $R_{b / \mu}$ and $R_{t / \mu}$, into the fit. However, doing so would require further assumptions about the model, as there are more undetermined parameters (couplings) than the mixing angle upon which these other observables depend. 

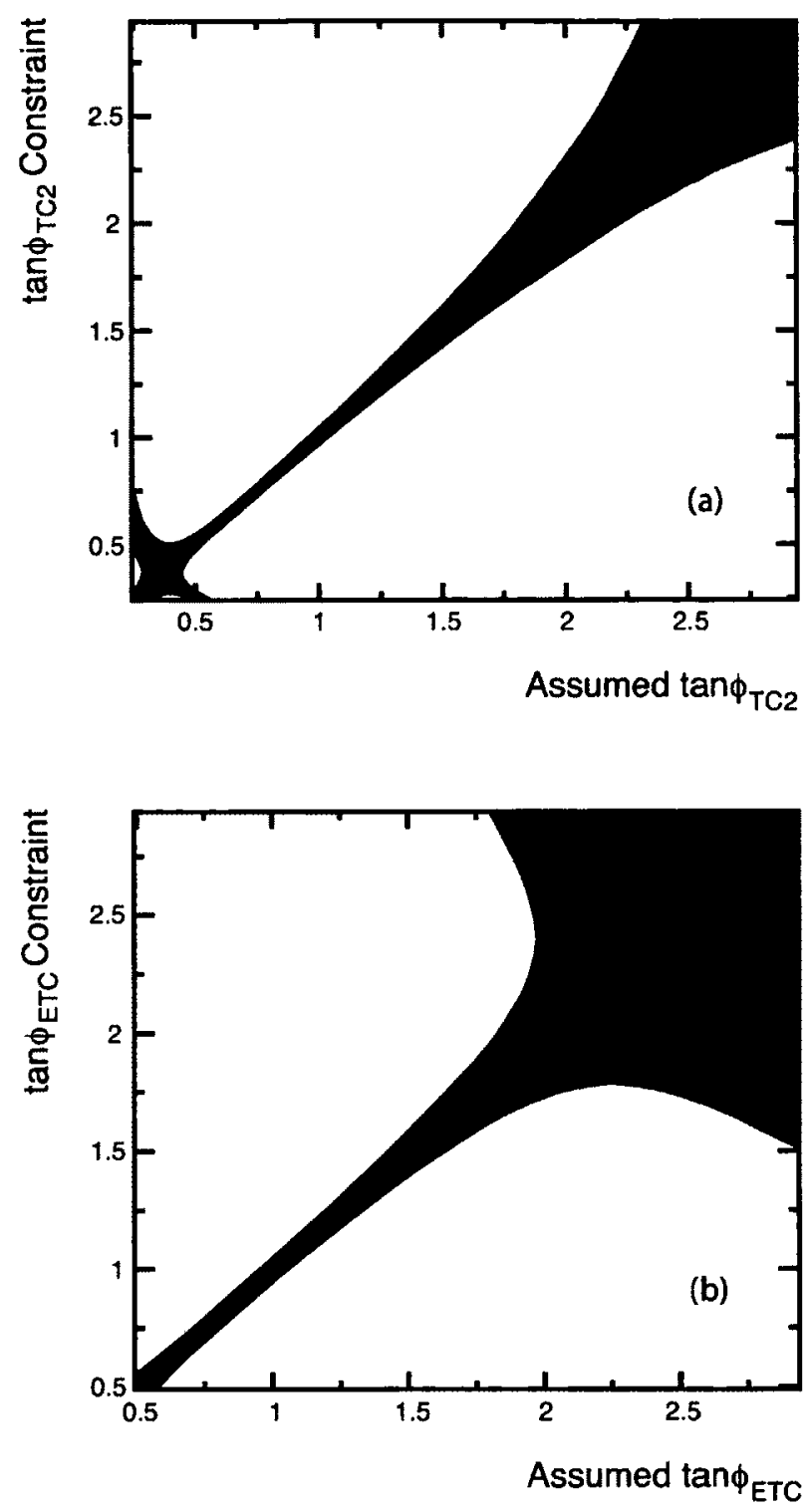

Figure 5.12: LHC measurement capability of the mixing parameter using $R_{\tau / \mu}$ for (a) TC2 and (b) ETC. The horizontal axis corresponds to the input parameter value and the verticle axis corresponds to the extracted value with 1- (grey) and 2- (black) $\sigma$ limits. $M_{Z^{\prime}}=1.5 \mathrm{TeV}$ and an integrated luminosity of $100 \mathrm{fb}^{-1}$ was assumed in these plots with the following kinematic cuts; $|\eta|<2.5,\left|M_{l^{+} l^{-}}-M_{Z^{\prime}}\right|<2.5 \Gamma_{Z^{\prime}}$, $p_{T}<0.3 M_{Z^{\prime}}$. 


\section{Part II}

\section{Exploring the Phenomenology of the Bestest Little Higgs Model at the LHC}




\section{Chapter 6}

\section{Model Details}

With the ongoing analysis of LHC data by the CMS and ATLAS collaborations, large regions of parameter space are being ruled out for many models of BSM physics [114-117]. However, the parameter space for Little Higgs (LH) models is strongly constrained by electroweak precision data $[118,119]$, and so the current LHC results have not had as significant of an effect on the Little Higgs parameter space.

Many LH models predict heavy top partners that are heavier than the mass of the new heavy gauge bosons, which leads to significant fine tuning in the Higgs potential $[120,121]$ as the lower limit on heavy gauge boson masses are pushed higher $[2,122]$. A recently proposed model, entitled the Bestest Little Higgs (BLH) [3], overcomes these difficulties by incorporating two distinct global symmetries that break to diagonal subgroups at different scales. The result is that the scales at which the heavy gauge bosons and top partners obtain their masses are distinct and thus the heavy gauge boson masses can be pushed above the already excluded mass range, while the heavy top partner masses remain below the upper bound from fine-tuning. The BLH model has two other attractive features: one, a custodial $S U(2)$ symmetry that protects the model from developing large deviations to EW precision measurements [118], and two, it is not challenged by the dangerous singlet problem [123] that is an unintended consequence of some Little Higgs models. These features further establish 
the viability of the model.

The Bestest Little Higgs model predicts many new fermion states, including four heavy top quark partners ( $+2 / 3$ charge), a heavy bottom partner $(-1 / 3$ charge) and an exotic $+5 / 3$ charge quark, and all of these can be relatively light without violating any current constraints. Since the top quark loop provides the largest divergent correction to the Higgs mass, the heavy quark sector is the most crucial to resolving the hierarchy problem and is the focus of the remainder of this thesis.

This chapter details the masses of the new particle states for all three sectors (scalar, gauge and fermion), and describes the particle interaction terms in the BLH Lagrangian. To determine the Feynman rules for the mass eigenstates, the full particle mixing given in Appendix $\mathrm{C}$ can be implemented with the provided interaction Lagrangian to solve for the vertex factors. Additionally, detailed considerations for the Coleman-Weinberg [124] contributions to the masses and interactions are given in Appendix B. These details expand upon those presented in the original paper by Schmaltz, Stolarski and Thaler [3], by examining the full potential in terms of 2HDM solutions that have already been developed in the literature.

\subsection{Mass Eigenstates and Particle Masses}

\subsubsection{Scalar Sector, Higgs Potential and Spontaneous Sym- metry Breaking}

The Bestest Little Higgs model is based on two independent non-linear sigma models: a $\Sigma$ field that breaks a global product group coset space $S O(6)_{A} \times S O(6)_{B}$ to a diagonal $S O(6)_{V}$ at scale $f(\approx 1 \mathrm{TeV})$, and a $\Delta$ field that breaks a global $S U(2)_{C} \times S U(2)_{D}$ symmetry to a diagonal $S U(2)$ at scale $F(\approx 3 \mathrm{TeV})$. 


\section{Non-Linear Sigma Field $\Sigma$}

At a scale $f$, the $\Sigma$ field develops a vacuum expectation value (vev), taken to be the identity, $\langle\Sigma\rangle=\mathbb{1}$, which breaks the global symmetry from $S O(6)_{A} \times S O(6)_{B} \rightarrow$ $S O(6)_{V}$. This leaves $n(n-1) / 2=15$ degrees of freedom in $S O(6)_{V}$, which are identified as two $S U(2)$ triplets $\left(\phi^{\alpha}\right.$ and $\left.\eta^{a}\right)$, two Higgs vectors as 4's of $S O(4)\left(h_{1}\right.$ and $h_{2}$ ), and a real singlet $(\sigma)$. The embedding of the scalar fields takes the form of:

$$
\Sigma=G\langle\Sigma\rangle G=e^{i \Pi_{o} / f} e^{i \Pi_{h} / f}\langle\Sigma\rangle e^{i \Pi_{h} / f} e^{i \Pi_{o} / f}
$$

where $\Pi_{o}$ and $\Pi_{h}$ are given by:

$$
\begin{aligned}
& \Pi_{o}=\phi_{a} T_{L}^{a}+\eta_{a} T_{R}^{a}+\left(\begin{array}{ccc}
0_{4 \times 4} & 0 & 0 \\
0 & 0 & i \sigma / \sqrt{2} \\
0 & -i \sigma / \sqrt{2} & 0
\end{array}\right) \\
& \Pi_{h}=\frac{i}{\sqrt{2}}\left(\begin{array}{ccc}
0_{4 \times 4} & h_{1} & h_{2} \\
-h_{1}^{T} & 0 & 0 \\
-h_{2}^{T} & 0 & 0
\end{array}\right)
\end{aligned}
$$

The triplet fields transform under their respective $S U(2)$ subgroups, $S U(2)_{L}$ and $S U(2)_{R}$, within the upper $S O(4)$ of the $S O(6)$ matrices, with corresponding generators, $T_{L}^{a}$ and $T_{R}^{a}$. These generators are listed in further detail in App. D.

An unstable Higgs potential is generated from terms involving two projection operators, $P_{5}=\operatorname{diag}(0,0,0,0,1,0)$ and $P_{6}=\operatorname{diag}(0,0,0,0,0,1)$, that explicitly break some of the symmetries protecting the Higgs. The Higgs quartic coupling is generated from:

$$
V=\frac{1}{4} \lambda_{65} f^{4} \operatorname{Tr}\left[P_{6} \Sigma P_{5} \Sigma^{T}\right]+\frac{1}{4} \lambda_{56} f^{4} \operatorname{Tr}\left[P_{5} \Sigma P_{6} \Sigma^{T}\right]
$$




$$
=\frac{f^{2} \lambda_{65}}{2}\left(\sigma-\frac{1}{\sqrt{2} f} h_{1}^{T} h_{2}+\ldots\right)^{2}+\frac{f^{2} \lambda_{56}}{2}\left(\sigma+\frac{1}{\sqrt{2} f} h_{1}^{T} h_{2}+\ldots\right)^{2}
$$

At this point, the triplet and Higgs fields are massless Goldstone bosons, but the $\sigma$ develops a large mass, $\left(\lambda_{56}+\lambda_{65}\right) f^{2}$, which is expected to be of order $1 \mathrm{TeV}$. Since the $\sigma$ has a large mass, it can be integrated out $\left(\sigma \rightarrow\left(\lambda_{65} h_{2}^{T} h_{1}-\lambda_{56} h_{1}^{T} h_{2}\right) / \sqrt{2} f\left(\lambda_{65}+\lambda_{56}\right)\right)$, which results in an effective quartic term:

$$
\frac{\lambda_{56} \lambda_{65}}{\lambda_{56}+\lambda_{65}}\left(h_{1}^{T} h_{2}\right)^{2} \rightarrow \frac{1}{2} \lambda_{0}\left(h_{1}^{T} h_{2}\right)^{2}
$$

To complete the scalar potential, small, explicit mass terms must be included that break the exact global symmetries by giving small masses to the Goldstone bosons. These are given by:

$$
\begin{gathered}
m_{56}^{2} f^{2} \operatorname{Tr}\left[P_{5} \Sigma P_{6}\right]+m_{65}^{2} f^{2} \operatorname{Tr}\left[P_{6} \Sigma P_{5}\right]-\frac{f^{2}}{4}\left(m_{5}^{2} \operatorname{Tr}\left[P_{5} \Sigma P_{5}\right]+m_{6}^{2} \operatorname{Tr}\left[P_{6} \Sigma P_{6}\right]\right) \\
-\frac{f^{2}}{4} m_{4}^{2} \operatorname{Tr}\left[\Delta^{\dagger} M_{26} \Sigma M_{26}^{\dagger}+\Delta M_{26} \Sigma^{\dagger} M_{26}^{\dagger}\right]
\end{gathered}
$$

The two Higgs fields, $h_{1}$ and $h_{2}$, obtain tree level mass terms, $m_{1}$ and $m_{2}$, from the explicit symmetry breaking terms in Eq. 6.6. The values of the Higgs masses, $m_{1}$ and $m_{2}$, also obtain log divergent contributions from loop diagrams (see App. B), which result in the expectation that $m_{2}>m_{1}$. Of note, the mass terms in Eq. 6.6 involving $m_{4}, m_{5}$ and $m_{6}$ could be more uniformly implemented via a term of the form $\frac{1}{4} f^{2} \operatorname{Tr}\left[\operatorname{diag}\left(m_{4}^{2} \mathbb{1}_{4 \times 4}, m_{5}^{2}, m_{6}^{2}\right) \Sigma\right]$. However, these terms are separated and the $2 \times 6$ matrix $M_{26}$ is included in order to preserve all the gauge symmetries, including hypercharge (which is associated with the diagonal $T_{R}^{3}$ generator) [3]. $M_{26}$ is provided in more detail in App. D.

Accounting for the effective interactions from integrating out the $\sigma$, a soft symmetry breaking, $B_{\mu}$ mass term $\left(B_{\mu} h_{1}^{T} h_{2}\right)$ results, given by:

$$
-2 \frac{\lambda_{65} m_{56}^{2}+\lambda_{56} m_{65}^{2}}{\lambda_{65}+\lambda_{56}} h_{1}^{T} h_{2} \rightarrow-B_{\mu} h_{1}^{T} h_{2}
$$


With this, the unstable scalar potential that results in spontaneous electroweak symmetry breaking (EWSB) can be summarized and written as:

$$
V_{E W S B}=\frac{1}{2} m_{1}^{2} h_{1}^{T} h_{1}+\frac{1}{2} m_{2}^{2} h_{2}^{T} h_{2}-B_{\mu} h_{1}^{T} h_{2}+\frac{\lambda_{0}}{2}\left(h_{1}^{T} h_{2}\right)^{2} .
$$

Loop contributions from Coleman-Weinberg calculations do introduce other terms of the form $\left(h_{1}^{T} h_{1}\right)^{2},\left(h_{2}^{T} h_{2}\right)^{2}$, and $\left(h_{1}^{T} h_{1}\right)\left(h_{2}^{T} h_{2}\right)$, but are expected to provide a negligible deviation to this EWSB potential. These are also covered in further detail in Appendix B.

This type of potential has been studied in great detail in the context of two Higgs doublet models (2HDM) [125]. The potential is minimized by a shift (vev) in the first components of both $h_{1}$ and $h_{2}$ :

$$
\begin{aligned}
& v_{1} \equiv\left\langle h_{11}\right\rangle=\frac{1}{\lambda_{0}} \frac{m_{2}}{m_{1}}\left(B_{\mu}-m_{1} m_{2}\right), \\
& v_{2} \equiv\left\langle h_{21}\right\rangle=\frac{1}{\lambda_{0}} \frac{m_{1}}{m_{2}}\left(B_{\mu}-m_{1} m_{2}\right) .
\end{aligned}
$$

The SM vev, $v_{E W}$, is related to the vevs $v_{1}$ and $v_{2}$ via the relation:

$$
\begin{gathered}
v_{E W}^{2} \equiv\left\langle h_{11}\right\rangle^{2}+\left\langle h_{21}\right\rangle^{2} \approx(246 \mathrm{GeV})^{2} \\
\tan \beta \equiv \frac{\left\langle h_{11}\right\rangle}{\left\langle h_{21}\right\rangle},
\end{gathered}
$$

where it is expected that $\tan \beta>1$, since $m_{2}>m_{1}$ due to contributions from top loops (see Appendix B).

In diagonalizing the resulting scalar mass matrix, the components of the $h_{1}$ and $h_{2}$ mix to form the SM Higgs field, $h^{0}$, two massive neutral scalars, $H^{0}$ and $A^{0}$, and two massive charged scalars, $H^{ \pm}$. These fields have masses given by:

$$
M_{h^{0}}^{2} \approx \lambda_{0} v_{E W}^{2} \sin ^{2} 2 \beta
$$




$$
\begin{aligned}
& M_{H^{0}}^{2} \approx M_{A^{0}}^{2}+\lambda_{0} v_{E W}^{2} \cos ^{2} 2 \beta \\
& M_{A^{0}}^{2}=M_{H^{ \pm}}^{2}=m_{1}^{2}+m_{2}^{2}
\end{aligned}
$$

In addition, three scalar fields, $G^{0}$ and $G^{ \pm}$, are eaten to give mass to the light electroweak gauge bosons.

\section{Non-Linear Sigma Field $\Delta$}

The $\Delta$ field transforms under a separate global $S U(2)_{C} \times S U(2)_{D}$ symmetry, and breaks to a diagonal $S U(2)$ at scale $F$, which is taken to be of order $3 \mathrm{TeV}$. The Nambu-Goldstone modes of the $\Delta$ non-linear sigma field are parameterized as:

$$
\begin{aligned}
\Delta & =e^{2 i \Pi_{d} / F} \\
\Pi_{d} & =\chi^{a} \frac{\tau^{a}}{2}
\end{aligned}
$$

where $\chi^{a}$ are the Nambu-Goldstone fields, and $\tau^{a}$ are the Pauli matrix generators of the $S U(2)$ symmetry. The $\phi^{a}$ triplet and the $\chi^{a}$ triplets mix to form a physical triplet, $\left(\phi^{\prime}\right)^{a} \equiv \phi_{\text {physical }}^{a}=\left(F \phi^{a}-f \chi^{a}\right) / \sqrt{f^{2}+F^{2}}$, and a triplet that is eaten to give mass to the new heavy gauge bosons, $\left(\chi^{\prime}\right)^{a} \equiv \phi_{\text {eaten }}^{a}=\left(f \phi^{a}+F \chi^{a}\right) / \sqrt{f^{2}+F^{2}}$.

The triplet scalars obtain small tree level masses from the terms introduced in Eq. 6.6, but also gain contributions from the Coleman-Weinberg (CW) potential that dominate their physical masses (see Appendix B). These loop contributions from the CW potential are expected to be cut off at the UV completion scale, which is taken to be $\Lambda \approx 4 \pi f$. The dominant contributions to the mass of the physical triplet states is given by:

$$
M_{\left(\phi^{\prime}\right)^{0}}^{2}=\frac{16}{3} F^{2} \frac{9 g_{A}^{2} g_{B}^{2}}{512 \pi^{2}} \log \left[\frac{\Lambda^{2}}{M_{W^{\prime}}^{2}}\right]+m_{4}^{2} \frac{f^{4}+F^{4}}{F^{2}\left(f^{2}+F^{2}\right)}
$$




$$
\begin{aligned}
M_{\left(\phi^{\prime}\right)^{ \pm}}^{2} & =\frac{16}{3} F^{2} \frac{9 g_{A}^{2} g_{B}^{2}}{512 \pi^{2}} \log \left[\frac{\Lambda^{2}}{M_{W^{\prime}}^{2}}\right]+m_{4}^{2} \frac{f^{4}+f^{2} F^{2}+F^{4}}{F^{2}\left(f^{2}+F^{2}\right)} \\
M_{\eta^{0}}^{2} & =m_{4}^{2} \\
M_{\eta^{ \pm}}^{2} & =m_{4}^{2}+2 f^{2} \frac{3 g_{Y}^{2}}{128 \pi^{2}} \frac{\Lambda^{2}}{f^{2}}
\end{aligned}
$$

Of note, the $\eta^{0}$ field remains light, with a mass proportional only to $m_{4}$, as it does not gain a mass contribution from CW loops.

\subsubsection{Gauge Bosons}

The gauge sector in the model arises from the gauging of an $S U(2)_{L A}$ subgroup of the $S O(6)_{A}$ symmetry and an $S U(2)_{L B}$ subgroup of the $S O(6)_{B}$ symmetry of $\Sigma$. By gauging the $S U(2)_{C} \times S U(2)_{D}$ symmetry for the $\Delta$ non-linear sigma field with the same $S U(2)_{A} \times S U(2)_{B}$ gauge group as the $\Sigma$, the new heavy gauge bosons develop masses proportional to a combination of $f$ and $F$. This causes the masses of the new gauge bosons to be large relative to other new particle states that have masses proportional to only $f$. The gauge invariant, non-linear sigma kinetic terms are given by:

$$
\mathcal{L}=\frac{f^{2}}{8} \operatorname{Tr}\left[D_{\mu} \Sigma^{\dagger} D^{\mu} \Sigma\right]+\frac{F^{2}}{4} \operatorname{Tr}\left[D_{\mu} \Delta^{\dagger} D^{\mu} \Delta\right]
$$

where the covariant derivatives are given by:

$$
\begin{aligned}
D \Sigma & =\partial \Sigma+i g_{A}\left(A_{1}^{a} T_{L}^{a}\right) \Sigma-i g_{B} \Sigma\left(A_{2}^{a} T_{L}^{a}\right)+i g_{Y}\left(B T_{R}^{3}\right) \Sigma-i g_{Y} \Sigma\left(B T_{R}^{3}\right) \\
D \Delta & =\partial \Delta+\frac{i}{2} g_{A}\left(A_{1}^{a} \tau^{a}\right) \Delta-\frac{i}{2} g_{B} \Delta\left(A_{2}^{a} \tau^{a}\right)
\end{aligned}
$$

The $S U(2)_{L A}$ symmetry is gauged by the triplet $A_{1}^{a}$, the $S U(2)_{L B}$ symmetry is gauged by the triplet $A_{2}^{a}$, and the hypercharge gauge field is given by $B$. The matrices $T_{L}^{a}$ 
and $\tau^{a}$ are the $6 \times 6$ and $2 \times 2$ generators of the $S U(2)_{L}$ symmetry group, and $T_{R}^{3}$ is the third component of the $6 \times 6$ generators of $S U(2)_{R}$.

When $\Sigma$ and $\Delta$ obtain their vevs, $f$ and $F$, the $A_{1}^{a}$ and $A_{2}^{a}$ gauge fields mix to form a massless triplet $\left(A_{0}^{a}\right)$ and a massive triplet $\left(A_{H}^{0}\right)$, given by the relations:

$$
\begin{aligned}
& A_{0}^{a}=\cos \theta_{g} A_{1}^{a}+\sin \theta_{g} A_{2}^{a}, \\
& A_{H}^{a}=\sin \theta_{g} A_{1}^{a}-\cos \theta_{g} A_{2}^{a},
\end{aligned}
$$

where the mixing angle is given by the relationship $\tan \theta_{g}=g_{A} / g_{B}$, and is related to the EW gauge coupling, $g$, via $g^{-2}=g_{A}^{-2}+g_{B}^{-2}$.

After electroweak symmetry breaking, when $h_{1}$ and $h_{2}$ develop their vevs, the neutral component of $A_{0}$ and $A_{H}$ mix with the $B$ field to form a massless photon, the SM $Z^{0}$ boson, and a massive $Z^{\prime}$. In addition, the shift in $h_{1}$ and $h_{2}$ results in a small shift in the mixing between the charged components of $A_{0}$ and $A_{H}$ that results in the $A_{0}^{ \pm}$developing a small mass, which identifies it as the SM $W^{ \pm}$. In terms of the parameters of the model, the masses are given by:

$$
\begin{aligned}
M_{W}^{2} & =\frac{1}{4} v_{E W}^{2} g_{E W}^{2}\left(1-\frac{1}{12} \frac{v_{E W}^{2}}{f^{2}}\left(2+\frac{3 f^{2}}{f^{2}+F^{2}}\left(\sin ^{2} \theta_{g}-\cos ^{2} \theta_{g}\right)\right)\right) \\
M_{Z}^{2} & =\frac{1}{4} v_{E W}^{2}\left(g_{E W}^{2}+g_{Y}^{2}\right)\left(1-\frac{1}{12} \frac{v_{E W}^{2}}{f^{2}}\left(2+\frac{3 f^{2}}{f^{2}+F^{2}}\left(\sin ^{2} \theta_{g}-\cos ^{2} \theta_{g}\right)\right)\right) \\
M_{W^{\prime}}^{2} & =\frac{1}{4} \frac{g_{E W}^{2}}{\sin ^{2} \theta_{g} \cos ^{2} \theta_{g}}\left(f^{2}+F^{2}\right)-M_{W}^{2} \\
M_{Z^{\prime}}^{2} & =M_{W^{\prime}}^{2}+g_{Y}^{2} \frac{1}{48 f^{2}} v_{E W}^{2}\left(\frac{3 f^{2}}{f^{2}+F^{2}}\left(\sin ^{2} \theta_{g}-\cos ^{2} \theta_{g}\right)\right)
\end{aligned}
$$

At leading order, the masses of the EW gauge bosons reproduce the leading order masses in the SM, as expected, and higher order contributions are suppressed by factors of $v^{2} / f^{2}$. The heavy gauge bosons obtain masses proportional to $\sqrt{f^{2}+F^{2}}$, which is dominated by the larger $F$ value, as expected. 


\subsubsection{Heavy Fermions}

The Bestest Little Higgs model contains an expanded heavy fermion sector. To achieve a collective top Yukawa coupling, the model includes two $Q$ fields that transform under $S O(6)_{A}$ and two $U^{c}$ fields that transform under $S O(6)_{B}$. These are given by:

$$
Q=\left(\begin{array}{c}
\frac{1}{\sqrt{2}}\left(-Q_{a 1}-Q_{b 2}\right) \\
\frac{i}{\sqrt{2}}\left(Q_{a 1}-Q_{b 2}\right) \\
\frac{1}{\sqrt{2}}\left(Q_{a 2}-Q_{b 1}\right) \\
\frac{i}{\sqrt{2}}\left(Q_{a 2}+Q_{b 1}\right) \\
Q_{5} \\
Q_{6} \\
U^{c}=\left(\begin{array}{c}
-Q_{a 1}^{\prime} \\
i Q_{a 1}^{\prime} \\
Q_{a 2}^{\prime} \\
i Q_{a 2}^{\prime} \\
0 \\
\frac{1}{\sqrt{2}}\left(-U_{b 1}^{c}-U_{a 2}^{c}\right) \\
\frac{i}{\sqrt{2}}\left(U_{b 1}^{c}-U_{a 2}^{c}\right) \\
\frac{1}{\sqrt{2}}\left(U_{b 2}^{c}-U_{a 1}^{c}\right) \\
\frac{i}{\sqrt{2}}\left(U_{b 2}^{c}+U_{a 1}^{c}\right) \\
U_{5}^{c} \\
U_{6}^{c}
\end{array}\right) U_{5}^{\prime c}=\left(\begin{array}{c}
0 \\
0 \\
0 \\
0 \\
0 \\
U_{5}^{\prime c} \\
0
\end{array}\right)
\end{array}\right.
$$

In order to recover the SM hypercharge values $\left(T_{Y}\right)$, a $U(1)_{X}$ charge is included in the hypercharge generator, defined as $T_{Y}=T_{R}^{3}+T_{X}$. The $Q$ fields are assigned a charge of $T_{X}=2 / 3$ under the $U(1)_{X}$ symmetry, while the $U^{c}$ fields are assigned a charge of $T_{X}=-2 / 3$. 
The heavy quark Yukawa Lagrangian is given by:

$$
\mathcal{L}_{t}=y_{1} f Q^{T} S \Sigma S U^{c}+y_{2} f Q^{\prime T} \Sigma U^{c}+y_{3} f Q^{T} \Sigma U_{5}^{\prime c}+\text { h.c. }
$$

where the $S$ represents an $S O(6)$ matrix that respects the gauge symmetries and acts to flip the sign of the Higgs fields in $\Sigma$. It is given by $S=\operatorname{diag}(1,1,1,1,-1,-1)$.

In the limit that $v_{E W} \rightarrow 0$, the $Q_{a}$ and $Q_{a}^{\prime}$ doublets mix to form a heavy and a massless doublet, while $U_{5}^{c}$ and $U_{5}^{\prime c}$ mix to form a heavy and a massless singlet. This massless doublet and singlet are identified as the third generation quark doublet and right handed top quark singlet. The mass eigenstates in this limit are given by:

$$
\begin{gathered}
\tilde{Q}_{a}=\frac{y_{1}}{\sqrt{\left|y_{1}\right|^{2}+\left|y_{2}\right|^{2}}} Q_{a}+\frac{y_{2}}{\sqrt{\left|y_{1}\right|^{2}+\left|y_{2}\right|^{2}}} Q_{a}^{\prime} \\
q_{3}=\frac{y_{2}^{*}}{\sqrt{\left|y_{1}\right|^{2}+\left|y_{2}\right|^{2}}} Q_{a}-\frac{y_{1}^{*}}{\sqrt{\left|y_{1}\right|^{2}+\left|y_{2}\right|^{2}}} Q_{a}^{\prime} \\
\tilde{U}_{5}^{c}=\frac{y_{1}}{\sqrt{\left|y_{1}\right|^{2}+\left|y_{3}\right|^{2}}} U_{5}^{c}+\frac{y_{3}}{\sqrt{\left|y_{1}\right|^{2}+\left|y_{3}\right|^{2}}} U_{5}^{\prime c} \\
u_{3}^{c}=\frac{y_{3}^{*}}{\sqrt{\left|y_{1}\right|^{2}+\left|y_{3}\right|^{2}}} U_{5}^{c}-\frac{y_{1}^{*}}{\sqrt{\left|y_{1}\right|^{2}+\left|y_{3}\right|^{2}}} U_{5}^{c}
\end{gathered}
$$

To convert from conjugate notation to anti-particle notation, the full Langranian is expanded out, with $Q U^{c} \rightarrow \bar{U} P_{L} Q$ and $U_{c}^{\dagger} Q^{\dagger} \rightarrow \bar{Q} P_{R} U$, to determine the partner states that form masses. In this case, the $\tilde{Q}_{a}$ and $U_{a}^{c}$ each form left and right handed components of an $S U(2)$ doublet, $T_{a}$, with charges $(+2 / 3,-1 / 3)$ and mass $f \sqrt{\left|y_{1}\right|^{2}+\left|y_{2}\right|^{2}} ; Q_{b}$ and $U_{b}^{c}$ each form left and right handed components of an exotic $S U(2)$ doublet $T_{b}$ with $(+5 / 3,+2 / 3)$ and mass $f\left|y_{1}\right| ; Q_{5}$ and $\tilde{U}_{5}^{c}$ form the left and right handed components of a singlet, $T_{5}$, with charge $+2 / 3$ and mass $f \sqrt{\left|y_{1}\right|^{2}+\left|y_{3}\right|^{2}}$; and $Q_{6}$ and $U_{6}$ form the left and right handed components of a singlet, $T_{6}$, with charge $+2 / 3$ and mass $f\left|y_{1}\right|$.

Assuming that the Yukawa couplings are real valued, these expressions can be simplified by re-parameterizing the three Yukawa couplings in terms of two mixing 
angles, $\theta_{12}$ and $\theta_{13}$, and an overall Yukawa strength, $y_{123}$. The two mixing angles are then defined by the Yukawa couplings as:

$$
\begin{aligned}
& \tan \theta_{12}=\frac{y_{1}}{y_{2}}, \\
& \tan \theta_{13}=\frac{y_{1}}{y_{3}} .
\end{aligned}
$$

And thus,

$$
\begin{aligned}
& y_{1}=\frac{y_{123}}{\cos \theta_{12} \cos \theta_{13}}, \\
& y_{2}=\frac{y_{123}}{\sin \theta_{12} \cos \theta_{13}}, \\
& y_{3}=\frac{y_{123}}{\cos \theta_{12} \sin \theta_{13}} .
\end{aligned}
$$

After the $h_{1}$ and $h_{2}$ fields undergo spontaneous symmetry breaking, the mass matrix is no longer diagonalized for the above eigenstates, and further mixing between the $+2 / 3$ charged quarks occurs. The mass terms in the Lagrangian can be written as components $M_{i j}$ of a mass matrix $\mathcal{L}_{t}=Q_{i} M_{i j} U_{j}$, where $Q_{i}$ and $U_{j}$ can be written as vectors $(\vec{Q}, \vec{U})$ such that $\mathcal{L}_{t}=\overrightarrow{Q^{T}} M \vec{U}$ in matrix notation. This mass matrix was diagonalized by introducing two unitary rotation matrices, $L_{D}$ and $R_{D}$, such that $\mathcal{L}_{t}=\vec{Q} L_{D} L_{D}^{\dagger} M R_{D} R_{D}^{\dagger} \vec{U}$ does not contain any off diagonal terms. Thus, $\vec{Q} L_{D}$ and $R_{D}^{\dagger} \vec{U}$ give the new mass eigenstates, and the diagonalized mass matrix squared is given by $M_{D}^{2}=L_{D}^{\dagger} M M^{\dagger} L_{D}=R_{D}^{\dagger} M^{\dagger} M R_{D}$. This was solved analytically by using perturbation theory, and expanding $M_{U}^{2}=M^{\dagger} M$ and $M_{Q}^{2}=M M^{\dagger}$ in powers of $v / f$, such that $M^{2}=\left.M^{2}\right|_{v_{E W} \rightarrow 0}+V^{2}$, where $V^{2}$ represents a non-diagonal matrix of perturbations to the diagonal tree level mass matrix, $\left.M^{2}\right|_{v_{E W} \rightarrow 0}$.

In the region where $y_{2} \neq y_{3}$, the mass eigenvalues can be expressed analytically 
(for eigenstate labels $t, T_{6}, T_{5}, T_{b}^{2 / 3}, T_{b}^{5 / 3}, T, B$ ) and are given by:

$$
\begin{aligned}
& M_{t}^{2}=\frac{9 y_{1}^{2} y_{2}^{2} y_{3}^{2} v_{E W}^{2} \sin ^{2} \beta}{\left(y_{1}^{2}+y_{2}^{2}\right)\left(y_{1}^{2}+y_{3}^{2}\right)}=y_{t}^{2} v_{E W}^{2} \sin ^{2} \beta \\
& M_{T}^{2}=\left(y_{1}^{2}+y_{2}^{2}\right) f^{2}+\frac{9 y_{1}^{2} y_{2}^{2} y_{3}^{2} v_{E W}^{2} \sin ^{2} \beta}{\left(y_{1}^{2}+y_{2}^{2}\right)\left(y_{2}^{2}-y_{3}^{2}\right)} \\
& M_{B}^{2}=\left(y_{1}^{2}+y_{2}^{2}\right) f^{2} \\
& M_{T_{5}}^{2}=\left(y_{1}^{2}+y_{3}^{2}\right) f^{2}-\frac{9 y_{1}^{2} y_{2}^{2} y_{3}^{2} v_{E W}^{2} \sin ^{2} \beta}{\left(y_{1}^{2}+y_{3}^{2}\right)\left(y_{2}^{2}-y_{3}^{2}\right)} \\
& M_{T_{6}}^{2}=M_{T_{b}^{2 / 3}}^{2}=M_{T_{b}^{5 / 3}}^{2}=y_{1}^{2} f^{2}
\end{aligned}
$$

When written in this form, it is clear that the top Yukawa coupling is related to the three Yukawa couplings in the model, and can be identified as $y_{t}=3 y_{123}$. This reduces the number of free parameters of the Yukawa couplings from three $\left(y_{i}\right.$ for $i=1,2,3$ or $\left.y_{123}, \theta_{12}, \theta_{13}\right)$ to two $\left(\theta_{12}\right.$ and $\left.\theta_{13}\right)$. This approximation of the diagonalized masses will be used throughout the remainder of this thesis.

It should be noted that when $y_{2} \approx y_{3}$ (or $\theta_{12} \approx \theta_{13}$ ), the $T_{5}$ and $T$ states become degenerate. Care must be taken when finding analytic expressions for the masses in the central and outer regions of $\theta_{12}-\theta_{13}$-space, as can be seen from the denominator of the second order terms in the masses of the $T$ and $T_{5}$ states in Eq. 6.23. The analytic expressions provided here are applicable only in the region where $\theta_{12} \neq \theta_{13}$.

In the central region of $\theta_{12}-\theta_{13}$-space, analytic expressions can be found by assuming $y_{3}=y_{2}+a v_{E W} / f$, for small values of $a$, which allows a further perturbative expansion in powers of $v_{E W} / f$. Alternatively, numerical methods can be employed to determine the masses over the full parameter range, which was used to produce Figures 6.1 and 6.2 .

For Figures 6.1 and 6.2, a large region of parameter space for the Yukawa couplings 

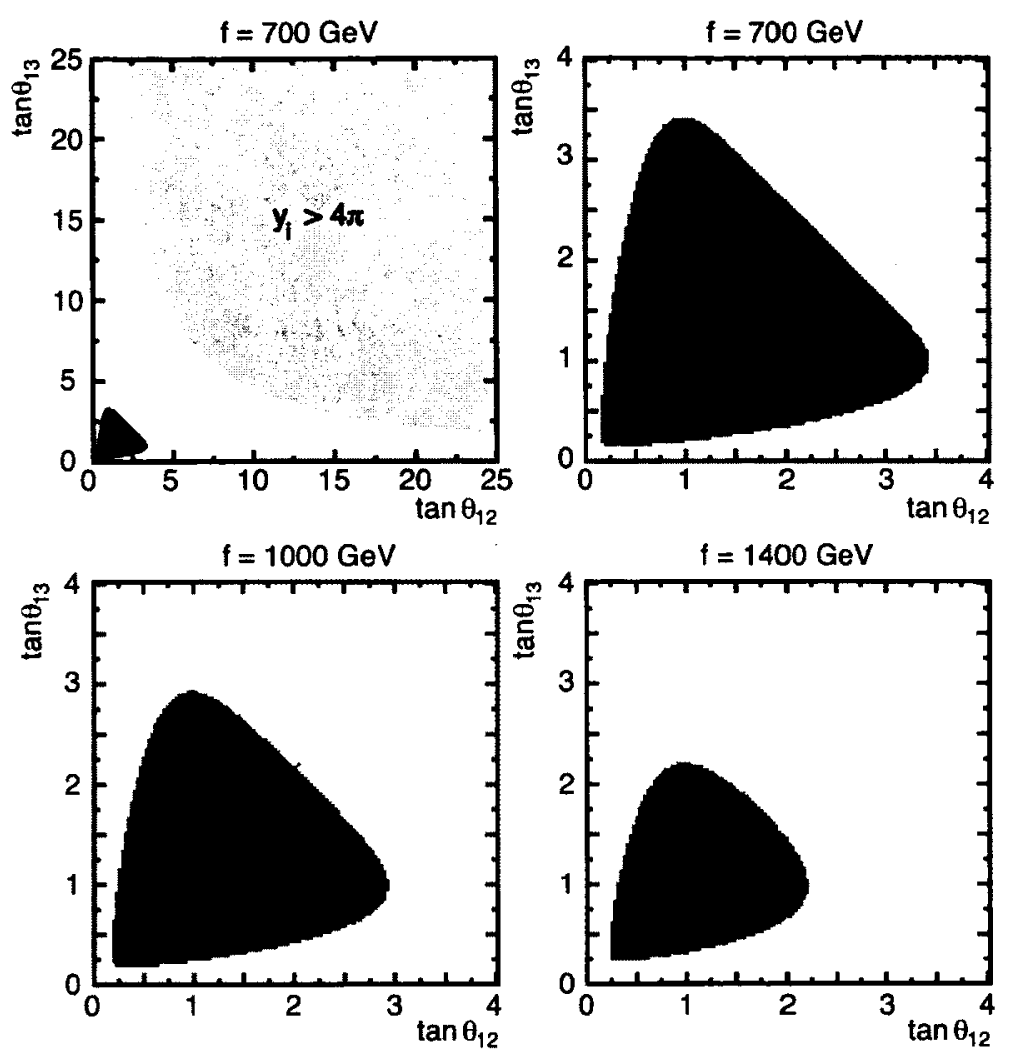

$M_{T_{6}}<M_{T_{5}}<M_{T}$

$M_{T_{6}}<M_{T}<M_{T_{5}}$

$M_{T}<M_{T_{6}}<M_{T_{5}}$

Figure 6.1: Constraints on $\theta_{12}$ and $\theta_{13}$ based on perturbativity and fine-tuning considerations, for $f=700,1000,1400 \mathrm{GeV}$, and $\tan \beta=\sqrt{3}$. The hierarchy of the heavy top-partners is shown in the legend at the bottom. The top two plots in the figure represent the same data but with different scales on the $x$ and $y$ axis.

was ruled out due to theoretical considerations: perturbation theory breaks down for large values of the Yukawa couplings, which gives an approximate constraint of $\left|y_{i}\right|<4 \pi$, and Little Higgs models are designed to overcome the Fine Tuning Problem, which suggests that the scale of heavy top partners must be less than about $2 \mathrm{TeV}$. The remaining parameter space after accounting for these two constraints is shown by the darker regions in Fig. 6.1.

From Fig. 6.1 it is clear that only for small values of $f$, and relatively large values of $\tan \theta_{12}$ and $\tan \theta_{13}$, is the $T$ state the lightest of the heavy fermions (see sliver in 




Figure 6.2: Masses of the heavy quarks in the BLH model, as $\tan \theta_{12}$ and $\tan \theta_{13}$ are varied. Other parameter values are $\tan \beta=\sqrt{3}$ and $f=1000 \mathrm{GeV}$.

upper right of Fig. 6.1). For the vast majority of the parameter space, the $T_{6}, T_{b}^{2 / 3}$ and the $T_{b}^{5 / 3}$ states are the lightest of the new quarks and have identical masses. Along the diagonal of $\tan \theta_{12} \approx \tan \theta_{13}$, Fig. 6.2 shows a sharp discontinuity in the masses that shifts the hierarchy from $M_{T_{6}}<M_{T_{5}}<M_{T}$ to $M_{T_{6}}<M_{T}<M_{T_{5}}$.

Figure 6.3 shows the mass difference between the $T$ quark and $B$ quark, where it is clear that $M_{T}-M_{B}<50 \mathrm{GeV}$, and $M_{B}$ is always between the masses of the $T$ and $T_{5}$ quarks. Since the $T$ and $T_{5}$ have identical charges and similar decay modes, this feature of the $B$ and $T$ masses may be useful in distinguishing between the two heaviest charge $+2 / 3$ states. However, the $T_{6}$ and the $T_{b}^{2 / 3}$ have identical masses and similar decay modes, and it is not possible to uniquely distinguish between these two states or determine their couplings independently. 


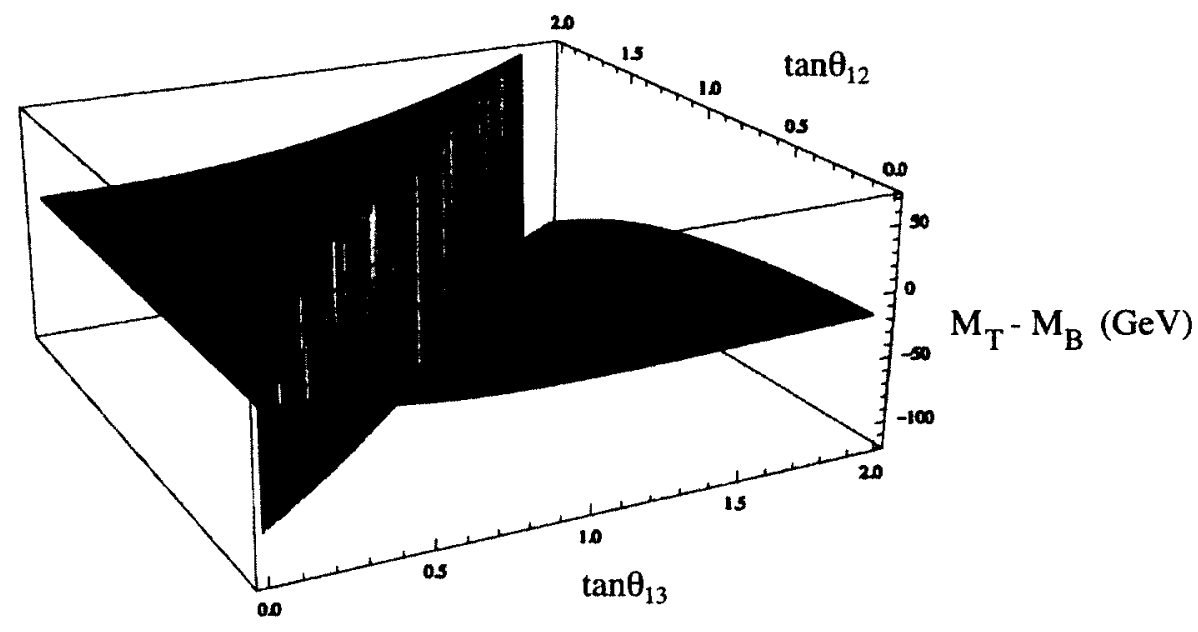

Figure 6.3: $\quad$ Mass difference between the $T$ state and $B$ state, as $\theta_{12}$ and $\theta_{13}$ are varied, for the same parameter values as Fig. 6.2.

\subsubsection{Light Fermions}

Light fermion doublets and singlets can be embedded in similar multiplets to the heavy fermions to reproduce the SM interactions. Fermion doublets are embedded as

$$
\begin{aligned}
& q_{i}^{T}=\frac{1}{\sqrt{2}}\left(-u_{i}, i u_{i}, d_{i}, i d_{i}, 0,0\right) \\
& \ell_{i}^{T}=\frac{1}{\sqrt{2}}\left(-\nu_{i}, i \nu_{i}, e_{i}, i e_{i}, 0,0\right)
\end{aligned}
$$

where the index $i$ refers to the generation, $i=1,2$, 3. Singlets are embedded as

$$
\begin{aligned}
& u_{i}^{c}=\left(0,0,0,0, u_{i}^{c}, 0\right) \\
& d_{i}^{c}=\left(0,0,0,0, d_{i}^{c}, 0\right) \\
& e_{i}^{c}=\left(0,0,0,0, e_{i}^{c}, 0\right)
\end{aligned}
$$

In the case of the singlets, the $u_{i}^{c}$ index only runs from $i=1,2$, since the third generation right handed quark is identified under the heavy fermion section above. 
Yukawa interactions involving the light fermions then take the form of:

$$
\mathcal{L}_{Y}=y_{u, i} f q_{i} \Sigma u_{i}^{c}+y_{d, i} f q_{i}\left(-2 i T_{R}^{2} \Sigma\right) d_{i}^{c}+y_{e, i} f \ell_{i}\left(-2 i T_{R}^{2} \Sigma\right) e_{i}^{c}
$$

where the $\left(-2 i T_{R}^{2}\right)$ results in charge conjugation for the down-type interactions.

The CKM matrix is assumed to come entirely from the down-type Yukawa couplings, and it may be possible to constrain the heavy Yukawa parameters further through examination of limits from meson factories. While the $b$ quark and $B$ quark states do not mix, and so no limits will arise from $D_{0}-\bar{D}_{0}$ mixing, mixing between the $t, T, T_{5}, T_{6}$ and $T_{b}^{2 / 3}$ states may result in limits found from $K_{0}-\bar{K}_{0}$ and $B_{d, s}-\bar{B}_{d, s}$ mixing constraints. This is beyond the scope of this investigation, and is left for future study.

\subsection{Interactions in the Bestest Little Higgs Model}

\section{Gauge Self Interactions}

Gauge self interactions arise from the following Lagrangian terms:

$$
\mathcal{L}=F_{1 \mu \nu} F_{1}^{\mu \nu}+F_{2 \mu \nu} F_{2}^{\mu \nu}
$$

where $F_{1,2}^{\mu \nu}$ are given by:

$$
\begin{aligned}
& F_{1}^{\mu \nu}=\partial^{\mu} A_{1}^{a \nu}-\partial^{\nu} A_{1}^{a \mu}+g_{A} \sum_{b} \sum_{c} \epsilon^{a b c} A_{1}^{b \mu} A_{1}^{c \nu} \\
& F_{2}^{\mu \nu}=\partial^{\mu} A_{2}^{a \nu}-\partial^{\nu} A_{2}^{a \mu}+g_{B} \sum_{b} \sum_{c} \epsilon^{a b c} A_{2}^{b \mu} A_{2}^{c \nu}
\end{aligned}
$$

In this form, the indices $a, b$ and $c$ run over the three gauge fields; $\epsilon^{a b c}$ is the antisymmetric tensor. 


\section{Scalar-Gauge Interactions}

Scalar-gauge interactions arise from the scalar kinetic term in the Lagrangian:

$$
\mathcal{L}=\frac{f^{2}}{8} \operatorname{Tr}\left[\left(D_{\mu} \Sigma\right)^{\dagger} D^{\mu} \Sigma\right]+\frac{F^{2}}{4} \operatorname{Tr}\left[\left(D_{\mu} \Delta\right)^{\dagger} D^{\mu} \Delta\right]
$$

The gauge covariant derivatives are given by:

$$
\begin{aligned}
D_{\mu} \Sigma & =i \sum_{a}\left(g_{A} A_{1 \mu}^{a} T_{L}^{a} \Sigma-g_{B} A_{2 \mu}^{a} \Sigma T_{L}^{a}\right)+i g_{Y} B_{3}\left(T_{R}^{3} \Sigma-\Sigma T_{R}^{3}\right) \\
D_{\mu} \Delta & =\frac{i}{2} \sum_{a}\left(g_{A} A_{1 \mu}^{a} \tau^{a} \Delta-g_{B} A_{2 \mu}^{a} \Delta \tau^{a}\right)
\end{aligned}
$$

In this notation, $T_{L}^{a}$ are the three $6 \times 6$ generators of the $S O(6)$ corresponding to the $S U(2)_{L}$ subgroup, and $T_{R}^{3}$ is the third component of the $S O(6)$ generators corresponding to the $S U(2)_{R}$ subgroup.

\section{Scalar-Fermion Interactions}

Scalar-fermion interactions arise from the Yukawa potential, given by:

$$
\begin{aligned}
\mathcal{L}_{t}= & y_{1} f Q^{T} S \Sigma S U^{c}+y_{2} f Q_{\Sigma}^{T} U^{c}+y_{3} f Q^{T} \Sigma U_{5}^{\prime c}+\sum_{i=1,2} y_{u, i} f q_{i} \Sigma u_{i}^{c} \\
& +\sum_{i=1,2,3}\left(y_{d, i} f q_{i}\left(-2 i T_{R}^{2} \Sigma\right) d_{i}^{c}+y_{e, i} f \ell_{i}\left(-2 i T_{R}^{2} \Sigma\right) e_{i}^{c}\right)+h . c .
\end{aligned}
$$

Expanding out $\Sigma$ results in mass terms from taking $\Sigma \rightarrow\langle\Sigma\rangle$, as shown above, and interaction terms of the form $f \bar{f} \phi$ and $f \bar{f} \phi \phi$, for fermion $f$ and arbitrary scalar field $\phi$.

\section{Gauge-Fermion Interactions}

Gauge-fermion interactions arise from the fermion kinetic terms in the Lagrangian. This is given by:

$$
\mathcal{L}=\sum_{i=1,2,3} i \bar{\sigma}_{\mu} \ell_{i}^{\dagger} D^{\mu} \ell_{i}+\sum_{i=1,2,3} i \bar{\sigma}_{\mu} e_{i}^{c \dagger} D^{\mu} e_{i}^{c}+\sum_{i=1,2} i \bar{\sigma}_{\mu} q_{i}^{\dagger} D^{\mu} q_{i}+\sum_{i=1,2} i \bar{\sigma}_{\mu} u_{i}^{c \dagger} D^{\mu} u_{i}^{c}
$$




$$
\begin{aligned}
& +\sum_{i=1,2} i \bar{\sigma}_{\mu} d_{i}^{c \dagger} D^{\mu} d_{i}^{c}+i \bar{\sigma}_{\mu} Q^{\dagger} D^{\mu} Q+i \bar{\sigma}_{\mu} Q^{\prime \dagger} D^{\mu} Q^{\prime}+i \bar{\sigma}_{\mu} U^{c \dagger} D^{\mu} U^{c}+i \bar{\sigma}_{\mu} U^{\prime c \dagger} D^{\mu} U^{\prime c} \\
& +i \bar{\sigma}_{\mu} b^{c \dagger} D^{\mu} b^{c}
\end{aligned}
$$

Since fermions have separate charges under the $S U(2)_{L A}, S U(2)_{L B}$ and $U(1)$ symmetries, the covariant derivatives are non-trivial. In order to reconstruct the correct electric charges, the $U(1)_{X}$ charges are given by $T_{X}^{+}=+2 / 3, T_{X}^{-}=-2 / 3, T_{X}^{d}=1 / 3$, and $T_{X}^{e}=1$. Using these values, the covariant derivatives are given by:

$$
\begin{aligned}
D^{\mu} \ell_{i} & =\sum_{a}\left(i g_{B} A_{2}^{a \mu} T_{L}^{a} \ell_{i}\right)+i g_{Y} B_{3}^{\mu} T_{R}^{3} \ell_{i} \\
D^{\mu} e_{i}^{c} & =i g_{Y} B_{3}^{\mu} T_{x}^{e} e_{i}^{c} \\
D^{\mu} q_{i} & =\sum_{a}\left(i g_{A} A_{1}^{a \mu} T_{L}^{a} q_{i}\right)+i g_{Y} B_{3}^{\mu}\left(T_{R}^{3}+T_{x}^{+}\right) q_{i} \\
D^{\mu} u_{i}^{c} & =i g_{Y} B_{3}^{\mu} T_{x}^{-} u_{i}^{c} \\
D^{\mu} d_{i}^{c} & =i g_{Y} B_{3}^{\mu} T_{x}^{d} d_{i}^{c} \\
D^{\mu} Q & =\sum_{a}\left(i g_{A} A_{1}^{a \mu} T_{L}^{a} Q\right)+i g_{Y} B_{3}^{\mu}\left(T_{R}^{3}+T_{x}^{+}\right) Q \\
D^{\mu} U^{\prime c} & =i g_{Y} B_{3}^{\mu} T_{x}^{-} U^{\prime c} \\
D^{\mu} Q^{\prime} & =\sum_{a}\left(i g_{A} A_{1}^{a \mu} T_{L}^{a} Q\right)+i g_{Y} B_{3}^{\mu}\left(T_{R}^{3}+T_{x}^{+}\right) Q^{\prime} \\
D^{\mu} U^{c} & =\sum_{a}\left(i g_{B} A_{2}^{a \mu} T_{L}^{a} U^{c}\right)+i g_{Y} B_{3}^{\mu}\left(T_{R}^{3}+T_{x}^{-}\right) U^{c} \\
&
\end{aligned}
$$


In this form, the indices on $\ell_{i}, e_{i}^{c}, q_{i}$, and $d_{i}^{c}$ run over the three generations, and the index of $u_{i}^{c}$ runs over the first two generations.

\section{Feynman Rules}

The Feynman rules for the BLH model can be reconstructed by expanding out the interaction terms in this section, and rotating the particle states to the eigenstates listed in App. C. These Feynman rules have been implemented in a MadGraph 4 [86] model file, which is available upon request. The Feynman rules were not included in this thesis as the specific details are unenlightening. 


\section{Chapter 7}

\section{Calculations}

Loops involving top quarks provide the largest divergent contribution to the Higgs mass, and thus understanding the heavy top sector and the cancellation of these loop contributions is a crucial step towards understanding the nature of a light Higgs [63]. In the Bestest Little Higgs model, loops involving heavy vector-like quarks cancel out the quadratic divergence from the top loops, resulting in a finite contribution to the Higgs mass that can be written in terms of the Yukawa couplings (see Eq. B.7), or in terms of the heavy quark masses. Thus, accessing either these couplings or the masses is necessary for understanding this cancellation.

Due to the energy needed to produce two heavy quarks, the pair production cross section drops rapidly with an increase in the mass of the heavy states. Thus, while pair production of heavy quarks through QCD interactions dominates the production of heavy quarks at relatively lower masses, single production through EW exchange of a $W$ boson becomes the dominant process for higher masses [126]. The point at which the single production becomes dominant depends on the branching ratio for the decay channel being considered, with smaller branching ratios reducing the measurable pair production cross section more significantly than with single production.

This chapter explores both the current discovery limits and the techniques needed to measure the masses, and thus determine the Yukawa couplings, in the BLH model. 
All Feynman rules for the new particle states in the BLH model were implemented in MadGraph4 [86], while branching ratios were calculated using the BRIDGE [127] software package and simulations were performed using MadGraph5 [128].

\subsection{Heavy Quark Discovery Limits}

Since the full range of parameter space for this model is quite large, two sample regions of parameter space that were chosen to characterize the range of mass splittings for the heavy quarks are used to explore the discovery reach. The two points in $\left(\theta_{12}\right.$, $\left.\theta_{13}\right)$-space that are considered are the non-isolated case $(\pi / 5, \pi / 3)$ and the isolated case $\left(\tan ^{-1}(0.5), \tan ^{-1}(0.9)\right)$, shown in Fig. 7.1. In both scenarios, the mass hierarchy for the heavy top partners is $M_{T_{6}}=M_{T_{b}^{2 / 3}}<M_{T_{5}}<M_{T}$, as the phenomenology is independent of whether the second lightest state is a $T$ or a $T_{5}$ other than a small difference in the measurable cross section due to different branching ratios. Fundamentally, the phenomenology is similar regardless of the mass hierarchy, assuming similar mass splittings.

These two scenarios are named based on the distinctive feature of their mass hierarchy. In the non-isolated scenario, the mass splitting between the $T_{6}$ and $T_{5}$ states is small, and so the primary decay modes of the $T_{5}$ are also to SM final states. In the isolated scenario, the mass splitting between the $T_{6}$ and $T_{5}$ states is larger, and so the $T_{5}$ decay modes include cascade decays, such as $T_{5} \rightarrow T_{b}^{5 / 3} W^{-}$. This is illustrated in Fig. 7.2. In the non-isolated scenario, then, $T_{5}$ pair production contributes to an event excess observed in the decay mode of interest, while $T_{5}$ pair production does not contribute significantly in the isolated scenario.

The primary decay modes considered for discovery of heavy quarks are to SM particles, since SM decays are well understood. This leaves two primary decay modes to consider for the heavy top partners: $T_{i} \rightarrow b W$ and $T_{i} \rightarrow t Z$. The couplings that 


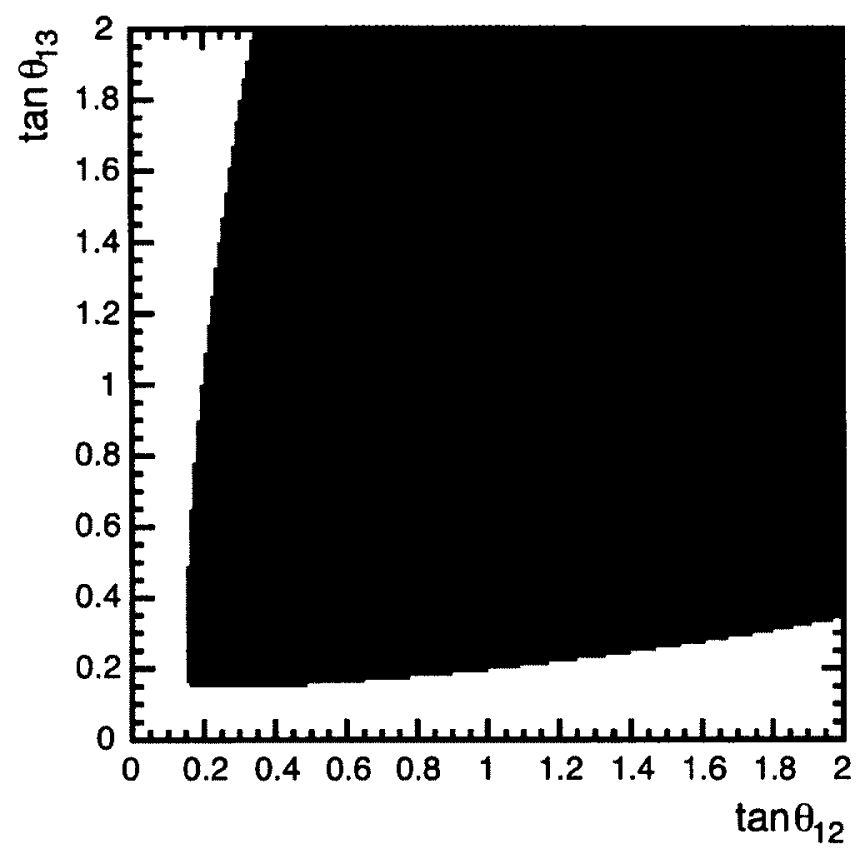

Figure 7.1: Enlarged view of the heavy quark parameter space showing placement of isolated (lower point) and non-isolated (upper point) scenarios, for $f=700 \mathrm{GeV}$. As evident from Fig. 6.1, this region of $\left(\theta_{12}, \theta_{13}\right)$ parameter space allows the greatest variability on the value of $f$ without violating any of the previously stated constraints.

lead to these decays do not arise at tree level, but rather at higher orders of $v / f$. For the $T_{b}^{2 / 3}$ state, the $t Z$ final state is the dominant decay mode, arising at $v / f$, while the $b W$ decay mode arises at $v^{2} / f^{2}$. Thus, for larger masses and consequently larger values of $f$, the $b W$ branching ratio falls off rapidly, as seen in Fig. 7.2.

For the $T_{6}$ state, both the $b W$ and $t Z$ decay modes arise from couplings proportional to $v / f$, but the $T_{6} t Z$ coupling is still smaller than the $T_{6} b W$ coupling for the scenarios considered. A similar situation arises for the $T_{5}$ state, where both $t Z$ and $b W$ couplings enter at the same order. However, for the isolated scenario, it is clear that kinematically accessible decays to other heavy quarks are favoured, and thus the SM branching ratios are suppressed. 

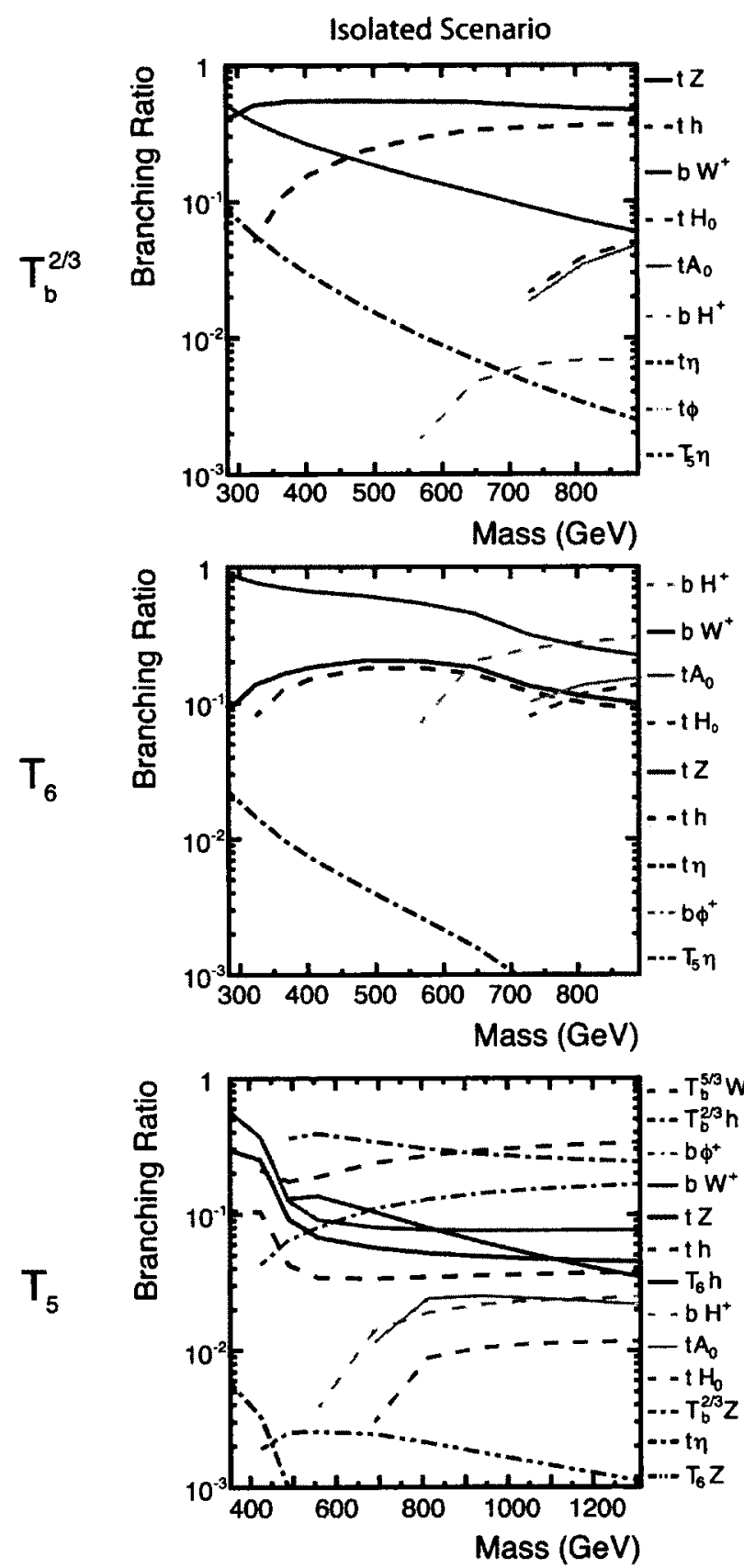


Figure 7.2: Branching ratios for the $T_{6}, T_{5}$ and $T_{b}^{2 / 3}$ states to all two-body final states with a significant branching ratio $(B R>0.1 \%)$. Branching ratios in the isolated scenario form the left plots, and the non-isolated scenario form the right plots. 
The numerical values in Fig. 7.2 that are relevant to the discovery reach calculations are listed in Tables 7.1 and 7.2 for the $b W$ and $t Z$ final states, respectively. In addition to the values of $f, \theta_{12}$ and $\theta_{13}$ that are listed, the following parameter values were used to calculate the branching ratios: $\tan \beta=\sqrt{3}, M_{h}=120 \mathrm{GeV}$, $M_{A_{0}, H^{ \pm}}=500 \mathrm{GeV}$, and $M_{\eta_{0}}=10 \mathrm{GeV}$. The scalar masses primarily affect the point at which decay modes involving these scalar states (such as $T_{6} \rightarrow A_{0} t$ ) become kinematically accessible. For the $A_{0}$ and $H^{ \pm}$scalars, their mass is expected to be large enough that it should not significantly affect the results, as it is not kinematically accessible for the mass ranges being examined here. However, the $\eta_{0}$ is light enough that decays to $t \eta_{0}$ are accessible, though the branching fraction to $t \eta_{0}$ is too small for variations in the value of $m_{\eta_{0}}$ to have any significant effect on the results. The mass of the $h$ state may affect the branching ratios of the heavy quarks in the mass region of interest, but recent results from ATLAS and CMS experiments suggest that a Higgs boson mass of $120 \mathrm{GeV}$ is not unreasonable [129,130]. Small changes to the mass of the Higgs boson have a negligible effect on these results.

CMS recently presented discovery limits for pair production of a heavy top-like quark at the LHC running at $\sqrt{s}=7 \mathrm{TeV}$, where the studies assumed scenarios with a $100 \%$ branching ratio to the $b W$ final state $[131]$ and with a $100 \%$ branching ratio to the $t Z$ final state [132], both for an integrated luminosity of $1.14 \mathrm{fb}^{-1}$.

These results can be applied to the Bestest Little Higgs model by comparing the cross section limits presented in the CMS studies to the total event excess for the $b \bar{b} W^{+} W^{-}$and $t \bar{t} Z Z$ final states that would arise from the heavy top-like quarks in the BLH model. The total cross section for the heavy top partner contribution is given by:

$$
\begin{gathered}
\sigma_{\text {tot }}=\sigma_{T_{5}}+\sigma_{T_{6}}+\sigma_{T_{b}^{2 / 3}} \\
\sigma_{T_{5}}=\sigma\left(M_{T_{5}}\right) B R\left(T_{5} \rightarrow q V\right)^{2}
\end{gathered}
$$


Table 7.1: Numerical summary of heavy quark branching ratios to the $b W$ final state.

\begin{tabular}{|c|c|c|c|c|c|}
\hline \multicolumn{6}{|c|}{ Non-Isolated } \\
\hline$f(\mathrm{GeV})$ & $M_{T_{6}, T_{b}^{2 / 3}}(\mathrm{GeV})$ & $M_{T_{5}}(\mathrm{GeV})$ & $\operatorname{BR}\left(T_{6}\right)$ & $\operatorname{BR}\left(T_{b}^{2 / 3}\right)$ & $\operatorname{BR}\left(T_{5}\right)$ \\
\hline 500 & 332.3 & 304.0 & 0.620 & 0.489 & 0.835 \\
\hline 600 & 398.8 & 396.5 & 0.566 & 0.413 & 0.739 \\
\hline 700 & 465.3 & 483.5 & 0.542 & 0.360 & 0.676 \\
\hline 800 & 531.8 & 567.6 & 0.524 & 0.321 & 0.614 \\
\hline 900 & 598.2 & 649.9 & 0.474 & 0.287 & 0.559 \\
\hline 1000 & 664.7 & 730.9 & 0.423 & 0.260 & 0.478 \\
\hline 1200 & 797.6 & 890.8 & 0.270 & 0.208 & 0.424 \\
\hline 1400 & 930.6 & 1048.7 & 0.218 & 0.169 & 0.402 \\
\hline \multicolumn{6}{|c|}{ Isolated } \\
\hline$f(\mathrm{GeV})$ & $M_{T_{6}, T_{b}^{2 / 3}}(\mathrm{GeV})$ & $M_{T_{5}}(\mathrm{GeV})$ & $\operatorname{BR}\left(T_{6}\right)$ & $\mathbf{B R}\left(T_{b}^{2 / 3}\right)$ & $\mathbf{B R}\left(T_{5}\right)$ \\
\hline 700 & 283.1 & 354.4 & 0.889 & 0.512 & 0.569 \\
\hline 800 & 323.5 & 424.8 & 0.768 & 0.385 & 0.370 \\
\hline 900 & 364.0 & 492.5 & 0.704 & 0.314 & 0.125 \\
\hline 1000 & 404.4 & 558.6 & 0.660 & 0.263 & 0.091 \\
\hline 1200 & 485.3 & 687.6 & 0.611 & 0.196 & 0.080 \\
\hline 1400 & 566.2 & 814.2 & 0.541 & 0.150 & 0.077 \\
\hline 1600 & 647.1 & 939.3 & 0.451 & 0.118 & 0.076 \\
\hline 1800 & 728.0 & 1063.4 & 0.316 & 0.092 & 0.076 \\
\hline
\end{tabular}


Table 7.2: Numerical summary of heavy quark branching ratios to the $t Z$ final state.

\begin{tabular}{|c|c|c|c|c|c|}
\hline \multicolumn{6}{|c|}{ Non-Isolated } \\
\hline$f(\mathrm{GeV})$ & $M_{T_{6}, T_{b}^{2 / 3}}(\mathrm{GeV})$ & $M_{T_{5}}(\mathrm{GeV})$ & $\mathbf{B R}\left(T_{6}\right)$ & $\mathbf{B R}\left(T_{b}^{2 / 3}\right)$ & $\mathbf{B R}\left(T_{5}\right)$ \\
\hline 400 & 265.9 & 198.5 & 0.029 & 0.115 & 0.000 \\
\hline 500 & 332.3 & 304.0 & 0.115 & 0.382 & 0.087 \\
\hline 600 & 398.8 & 396.5 & 0.149 & 0.440 & 0.224 \\
\hline 700 & 465.3 & 483.5 & 0.175 & 0.471 & 0.266 \\
\hline 800 & 531.8 & 567.6 & 0.187 & 0.486 & 0.268 \\
\hline 900 & 598.2 & 649.9 & 0.182 & 0.493 & 0.257 \\
\hline 1000 & 664.7 & 730.9 & 0.170 & 0.492 & 0.227 \\
\hline 1200 & 797.6 & 890.8 & 0.116 & 0.480 & 0.206 \\
\hline 1400 & 930.6 & 1048.7 & 0.098 & 0.464 & 0.198 \\
\hline \multicolumn{6}{|c|}{ Isolated } \\
\hline$f(\mathrm{GeV})$ & $M_{T_{6}, T_{b}^{2 / 3}}(\mathrm{GeV})$ & $M_{T_{5}}(\mathrm{GeV})$ & $\mathbf{B R}\left(T_{6}\right)$ & $\mathbf{B R}\left(T_{b}^{2 / 3}\right)$ & $\mathbf{B R}\left(T_{5}\right)$ \\
\hline 600 & 242.7 & 279.4 & 0.000 & 0.000 & 0.101 \\
\hline 700 & 283.1 & 354.4 & 0.089 & 0.400 & 0.296 \\
\hline 800 & 323.5 & 424.8 & 0.137 & 0.508 & 0.252 \\
\hline 900 & 364.0 & 492.5 & 0.163 & 0.538 & 0.091 \\
\hline 1000 & 404.4 & 558.6 & 0.183 & 0.550 & 0.068 \\
\hline 1200 & 485.3 & 687.6 & 0.205 & 0.550 & 0.057 \\
\hline 1400 & 566.2 & 814.2 & 0.203 & 0.544 & 0.052 \\
\hline 1600 & 647.1 & 939.3 & 0.182 & 0.535 & 0.049 \\
\hline 1800 & 728.0 & 1063.4 & 0.133 & 0.506 & 0.047 \\
\hline
\end{tabular}




$$
\begin{aligned}
\sigma_{T_{6}} & =\sigma\left(M_{T_{6}}\right) B R\left(T_{6} \rightarrow q V\right)^{2} \\
\sigma_{T_{b}^{2 / 3}} & =\sigma\left(M_{T_{b}^{2 / 3}}\right) B R\left(T_{b}^{2 / 3} \rightarrow q V\right)^{2}
\end{aligned}
$$

where $q=t, b$ and $V=Z, W^{+}$for the two final states considered. The cross sections are calculated using a fit of the NLO heavy quark production cross section data listed in Table $\mathrm{V}$ from Berger and Cao [126] for $\sqrt{s}=7 \mathrm{TeV}$, similar to the CMS analysis. The cross sections in [126] were found using the CTEQ6.6M PDF set [133].

Since the cross sections from multiple quark states are combined, the cross section limit $\left(L_{c}\right)$ must be adjusted to account for the combined kinematics of heavy quarks with different masses $\left(M_{T_{5}}>M_{T_{6}}\right)$. This is approximated as a sum of the limits provided at the masses of the two lightest states $\left(M_{T_{5}}\right.$ and $\left.M_{T_{6}}\right)$, weighted by the ratio of cross sections, as shown in Eq. 7.2.

$$
L_{c}\left(M_{\min }\right)=\frac{\sigma_{T_{6}}}{\sigma_{t o t}} L\left(M_{T_{6}}\right)+\frac{\sigma_{T_{b}^{2 / 3}}}{\sigma_{t o t}} L\left(M_{T_{b}^{2 / 3}}\right)+\frac{\sigma_{T_{5}}}{\sigma_{t o t}} L\left(M_{T_{5}}\right)
$$

The mass of the $T_{6}$ and $T_{b}^{2 / 3}$ quarks are identical up to order $v_{E W}^{2} / f^{2}$, and so the first two contributing terms for the limit, on the right hand side of Eq. 7.2, are identical. Since the pair production cross section drops rapidly with the increasing mass of the heavy quark, $\sigma_{T_{5}}$ is small compared to $\sigma_{T_{6}}$ and so the limit is validly approximated as $L_{c} \approx L\left(M_{T_{6}}\right)$, taken from the CMS studies.

\subsection{Measuring Masses and Determining Yukawa Couplings}

As discussed in Section 6.1.3, there are three Yukawa couplings $\left(y_{1}, y_{2}\right.$ and $\left.y_{3}\right)$ that fix the masses and mixing of the heavy quarks. By identifying the top Yukawa coupling 
$y_{t}$ in terms of $y_{123}$, the number of degrees of freedom is reduced by one, such that the top sector can be parameterized by $y_{t}$ and two arbitrary mixing angles, $\theta_{12}$ and $\theta_{13}$.

Determining these mixing angles, and thus the Yukawa couplings, is important for two reasons - establishing the parameters of the model and determining the degree of cancellation in the Higgs sector. In Supersymmetric models, for example, loops involving superpartners of SM particles naturally cancel out the Higgs mass divergences [134]. In Little Higgs models, the divergent contributions cancel due to the collective symmetry breaking principle [135], and a finite contribution remains. Establishing a finite mass contribution in agreement with the measured Higgs mass would establish the viability of a Little Higgs scenario.

As discussed by Han et al. [63], it would be preferable to measure Yukawa couplings in a model-independent way in order to determine the Higgs cancellation. However, this may not be possible at the LHC. Instead, the Yukawa couplings must be explored in a model-dependent fashion, ideally by over constraining the parameter space. The relationship between the heavy quark masses and the cancellation in the Higgs sector is given explicitly in App. B in terms of the Yukawa couplings, but in terms of the masses, this finite contribution can we written as:

$$
\delta M_{h} \approx-\frac{9}{16 \pi} \frac{M_{B}^{2}\left(M_{T}^{2}-M_{B}^{2}\right)}{v_{E W}^{2} \sin ^{2} \beta} \log \left(\frac{M_{B}^{2}}{M_{T_{5}}^{2}}\right) .
$$

Thus, to determine the contribution of the heavy quark loops to the Higgs mass, either a minimum of a measurement of all three of the $B, T$ and $T_{5}$ masses is necessary, or else another way to measure the Yukawa mixing angles is necessary to calculate the heavy quark contribution.

The focus of this section is to explore some of the capabilities of the LHC to measure heavy quark masses, and to determine the usefulness of the results. Since either the $T$ or the $T_{5}$ ends up being much heavier than the others, it is likely that other measurements will be needed to constrain the parameter space. This could 
include measuring the single $\left(T_{i}+j\right)$ or associated $\left(T_{i}+h\right)$ production cross section of heavy top partners, or by determining branching ratios of the heavy quarks. Such measurements may be the focus of future work.

Because the heavy bottom quark, $B$, and the exotic $+5 / 3$ charge quark, $T_{b}^{5 / 3}$, do not mix with SM fermions, their masses do not obtain contributions at higher orders in the expansion of $v / f$. Thus, the ratio of the masses of these quarks does give a direct way to determine the mixing angle $\theta_{12}$, given that $M_{T_{b}^{5 / 3}} / M_{B}=\left|\sin \theta_{12}\right|$. Thus, knowing $M_{T_{b}^{5 / 3}}\left(=M_{T_{b}^{2 / 3}}=M_{T_{6}}\right)$ and $M_{B}$ are important features of the analysis.

Both the $B$ and $T_{b}^{5 / 3}$ quark states have similar decay chains, $B \rightarrow t W^{-} \rightarrow$ $b W^{+} W^{-}$and $T_{b}^{5 / 3} \rightarrow t W^{+} \rightarrow b W^{+} W^{+}$. When pair produced, the final state signatures are identical: $B \bar{B} \rightarrow b W^{+} W^{-} \bar{b} W^{-} W^{+}$and $T_{b}^{5 / 3} \bar{T}_{b}^{5 / 3} \rightarrow b W^{+} W^{+} \bar{b} W^{-} W^{-}$. Since the exotic quark, $T_{b}^{5 / 3}$ has the same mass as the two lightest top-partners, $T_{6}$ and $T_{b}^{2 / 3}$, measuring the mass of the lightest top partner states can be used to determine which mass corresponds to which of the quark states - the exotic or the bottom partner - from the expected dual peak in this channel. However, distinguishing two $b$-jets and four $W$ bosons still presents a significant challenge due to the stringent tagging methods needed to veto the large backgrounds, and so it is expected that this measurement will result in a low event rate at the LHC.

Alternatively, in scenarios where the $B$ mass is large and cannot be measured, the $T_{5}$ state is typically light enough to be measured. The mass of the $T_{5}$ state is dependent on $y_{1}^{2}+y_{3}^{2}$ at leading order, which provides information on the other mixing angle, $\theta_{13}$. However, for masses light enough to be measurable at the LHC (and thus, small enough $f)$, the higher order $\left(v^{2} / f^{2}\right)$ correction may provide a significant modification from this leading order mass and obscure an accurate measurement of $\theta_{13}$. Instead, a model dependent global fit of all the masses would be ideal, and give a better indication of the values of the mixing angles, as well as isolate the value of $f$. 


\subsubsection{Heavy Top-Partners}

Measuring the masses of the heavy quarks can be done by fitting a template line-shape to the invariant mass distribution of events with the specific decay signature. This technique has been applied to the measurement of the top quark mass at the LHC, which has determined the top mass to an accuracy of $2 \%$ [136] ( $\pm 0.5 \%$ statistical, $\pm 1.5 \%$ systematic). For the measurement of the top quark mass using this method, the semi-leptonic final state is used, where one $W$ decays hadronically and the other decays leptonically. While this results in missing information due to the $E_{T}$ from the neutrino, the added benefit of this final state is that the backgrounds are significantly smaller.

To correctly identify which $b$-jet should be associated with the hadronic $W$ decay for semi-leptonic top quark pair production events at the LHC, two methods can be used: selecting only highly boosted events, where the $\Delta R$ separation between the $b$ and $W$ candidates can be used to discriminate the correct pairing, or by employing a $\chi^{2}$ test for correctly pairing the $t \rightarrow b W$ candidate based on the known top mass. For a heavy top partner of unknown rest mass, this type of identification is impossible. The increased phase space available to the decay products of the top partner means that $\Delta R$ is a poor discriminator of the decay product pairing. Additionally, the unknown mass means that a $\chi^{2}$ technique for a system with missing energy is not possible. Instead, the fully hadronic state can be used since the $W$ 's are expected to be boosted, and the invariant mass of both possible $b W$ pair combinations (e.g.: $b_{1} W_{1}$ vs $\left.b_{1} W_{2}\right)$ can be used to improve the determination of the correct permutation.

To identify the most likely correct $b$ candidate and $W$ candidate pairings, the invariant masses of both $b$ candidate and $W$ candidate combinations were determined, $M_{b W}^{i j}$, where $i$ refers to the index of the $b$ candidate and $j$ for the $W$ candidate. The candidates are assumed correctly paired for the smallest absolute valued difference 
between the invariant mass of the two $b W$ pairs, i.e. a measurement of $\left|M_{b W}^{11}-M_{b W}^{22}\right|<$ $\left|M_{b W}^{12}-M_{b W}^{21}\right|$ would pair tagged jets 11 and 22 . Since the heavy quarks are assumed to be pair produced, the correct pairing should result from the permutation in which the $b W$ pairs have the most similar invariant masses, and an incorrect pairing should result in distinct invariant masses.

The full $b \bar{b} W^{+} W^{-}$process is simulated in MadGraph 5 [128] using weighted events. Instead of further decaying the $W$ bosons hadronically, they are treated as fat jet objects, and the event weight is further reduced by the hadronic branching ratio. Furthermore, a $5 \% p_{T}$ resolution is applied on all hadronic final states to simulate the effects of energy resolution smearing on the results.

Furthermore, these events are binned based on the maximum of the two invariant mass values for each heavy quark candidate $\left(\operatorname{Max}\left(M_{b W}^{1}, M_{b W}^{2}\right)\right)$. This distribution is characterized by a large peak cross section arising from $t \bar{t}$ events at a low $b W$ invariant mass, and smaller peaks for each of the heavy top-partners. Neglecting the top peak events, the binned background events on either side of the top partner peaks was subtracted based on a fit to a function of the form:

$$
\log (N(M))=A M^{-2}+B M^{-1}+C+D M+E M^{2}
$$

for $N$ events at invariant mass $M$, and arbitrary fit coefficients $A, B, C, D$, and $E$. The remaining background subtracted peak(s) were then fit to a Breit-Wigner lineshape. The central mass for each peak was thus determined, as well as an estimation of the width. However, the observed width does not represent the decay width of the heavy top partner due to the smearing of the peak arising from the jet energy resolution uncertainty. 


\section{Backgrounds}

For the fully hadronic final state, charge tagging is more difficult. Thus, all backgrounds are simulated in a "charge-blind" fashion - $b \bar{b}$ events are considered along side $b b$ and $\bar{b} \bar{b}$, as well as $W^{+} W^{-}$along side $W^{+} W^{+}$and $W^{-} W^{-}$. The light jet background is reduced by demanding two $b$-tagged jets as well as two hadronic $W$ fat-jets [96]. Light jets faking a $b$ or a hadronic $W$ are included, as per the discussion in subsection 7.2 .3 on tagging efficiency.

The full list of backgrounds simulated in MadGraph are, in order of size:

- $p p \rightarrow b(\bar{b}) \bar{b}(b) W^{ \pm} W^{ \pm}+X$

- $p p \rightarrow b(\bar{b}) \bar{b}(b) j j+X$

- $p p \rightarrow j b(\bar{b}) \bar{b}(b) W^{ \pm}+X$

- $p p \rightarrow j j j b(\bar{b})+X$

- $p p \rightarrow j j j j+X$

- $p p \rightarrow j b(\bar{b}) W^{ \pm} W^{ \pm}+X$

- $p p \rightarrow j j j W^{ \pm}+X$

- $p p \rightarrow j j b(\bar{b}) W^{ \pm}+X$

- $p p \rightarrow j j W^{ \pm} W^{ \pm}+X$

Other possible mistagged backgrounds involving hadronically decaying $Z$ bosons instead of $W$ bosons are neglected, as these backgrounds are suppressed by small EW couplings and are thus significantly smaller than backgrounds involving only QCD vertices. 


\subsubsection{Heavy Bottom Partner and Exotic Quark}

The primary decay mode of both the $B$ and the $T_{b}^{5 / 3}$ is to the $t W$ final state, assuming that the mass difference between the heavy quark and the top quark is smaller than the mass of the charged scalars in the model (assumed to be of order $500 \mathrm{GeV}$ ). The signal for observing pair production of these states is then $b \bar{b} W^{+} W^{+} W^{-} W^{-}$.

For a "cut-and-count" method of discovery of these quarks, ATLAS is currently considering one-lepton and two-lepton decay modes, with three or two hadronically decaying $W \mathrm{~s}$, respectively [137]. Similar to the discussion on the top partner measurements, leptonic $W$ decays result in a challenge to reconstructing which top candidate is associated with the leptonic $W$. While semileptonic decay modes are useful for identifying an excess of events in cut-and-count techniques, they are difficult to use for observing an invariant mass peak.

With each heavy quark decaying to a $b$ quark and two hadronic $W$ 's, matching the $b W W$ combination that arises from each original heavy quark is accomplished by minimizing the following $\chi^{2}$ :

$$
\chi^{2}=\frac{\left(M_{b_{1} W_{i}}-M_{t}\right)^{2}}{\left(0.1 M_{t}\right)^{2}}+\frac{\left(M_{b_{2} W_{j}}-M_{t}\right)^{2}}{\left(0.1 M_{t}\right)^{2}}+\frac{\left(M_{b_{1} W_{i} W_{k}}-M_{b_{2} W_{j} W_{m}}\right)^{2}}{\delta_{b W W}}
$$

for $i, j, k, m=1,2,3,4$ (where $i \neq j \neq k \neq m$ ) as all possible combinations of the $W$ pairings. This technique is based on reconstructing the two intermediate top quark states, and matching the invariant masses of the two originating heavy quarks.

Due to smearing effects, the invariant mass of the intermediate tops will have an apparent width larger than the actual width, and thus a value of $0.1 M_{t}$ is used to weight the contribution of the top quark mass measurement in the $\chi^{2}$. For the final pairing of the $t W$ candidates to match the masses of the originating heavy quarks, the value of $\delta_{b W W}$ was varied and compared to an idealized tagging. The result was found to be relatively insensitive to variations in moderate values of $\delta_{b W W}$, and a value of $20 \mathrm{GeV}$ was used. 


\section{Backgrounds}

Estimations of the backgrounds for this signal are difficult. Even with all $W$ 's treated as fat jet objects, this signal is a six-body decay with thousands of contributing diagrams for the many possible mistagged backgrounds. As a result, AlpGen [138$140]$ is used to simulate the backgrounds for these processes, as MadGraph is unable to solve the massive number of diagrams for these backgrounds.

For actual studies of these many body processes, ATLAS and CMS typically use data-driven techniques for determining the backgrounds [141]. Without access to this data, Monte-Carlo techniques must be used to estimate the largest contributions to the backgrounds. While the reducible backgrounds are large compared to the signal, $b$-jet tagging significantly reduces those backgrounds with mistagged light jets as $b$-jets, as evidenced from the heavy top partner analysis. These backgrounds are thus neglected. However, because events with light jets faking a $W$ boson arise from strongly coupled interactions, instead of weakly coupled, and because of the greater combinatorics for purely light jet events, backgrounds that fake all four $W$ bosons are still significant and are included in this analysis. As a result, the following list of backgrounds are included:

- $p p \rightarrow t(\bar{t}) \bar{t}(t) W^{ \pm} W^{ \pm}+X$

- $p p \rightarrow t(\bar{t}) \bar{t}(t) j j+X$

- $p p \rightarrow b \bar{b} j j j j+X$

\subsubsection{Tagging Efficiency}

In a recent paper on jet substructure techniques for boosted, massive, hadronic objects, it was determined that the efficiency for correctly identifying a hadronic $W$ $\left(\epsilon_{W}\right)$ is between $40 \%$ and $70 \%$, based on the transverse momentum of the event, for 
$p_{T}(W)>200 \mathrm{GeV}$, with a peak efficiency occurring at $p_{T}(W) \approx 300 \mathrm{GeV}[96]$. The mistag rate $\left(\phi_{W}\right)$ for light jets over the same momentum range is between $5 \%$ and $16 \%$, and also peaks at the same point.

In ATLAS papers that employ $b$ tagging techniques, the efficiency $\left(\epsilon_{b}\right)$ is assumed between $60 \%$ and $70 \%$ [95], with a fake rate $\left(\xi_{b}\right)$ between $0.1 \%$ and $1 \%$ for $b$-jets with a transverse momentum between 50 and $500 \mathrm{GeV}$. For simplicity, light jet backgrounds are assumed to include jets arising from charm quarks, and no effort is made to differentiate them. These tagging efficiencies are implemented into this heavy quark analysis in a $p_{T}$ dependent way, using the distributions provided in [95] and [96].

For events where light jets are assumed to be faking both a $b$ and a $W$, a random number, $r$, is generated in the range of $[0,1]$, and the light jet is assigned as a $b$ if $r<\xi_{b} /\left(\epsilon_{b}+\phi_{W}\right)$, and a $W$ if not. This ensures that any possible biasing in the ordering of the decay products in MadGraph and AlpGen does not affect the distributions of the backgrounds.

Overall, the tagging is used to generate the lists of states used for determining the $b W$ and $b W W$ permutations, and the weight of the simulated event is reduced by a factor for each of the tagging probabilities for each $b$-jet and $W$, determined at the appropriate transverse momentum. The weight of simulated events with light jets is similarly reduced by a factor of the fake rate for each mistagged $b$ and $W$. 


\section{Chapter 8}

\section{Results}

\subsection{Discovery Limits}

The numerical results for the excess $b \bar{b} W^{+} W^{-}$and $t \bar{t} Z Z$ cross sections due to pair production of $T_{6}, T_{b}^{2 / 3}$ and $T_{5}$ quarks, as described in Eq. 7.1, are listed in Tables 8.1 and 8.2, for $7 \mathrm{TeV}$ collisions at the LHC. These results are plotted in Fig. 8.1 for the $b W$ channel, and Fig. 8.2 for the $t Z$ channel, versus the mass of the lightest of the heavy top partners (the lightest state is $T_{6}$ and $T_{b}^{2 / 3}$ over the range of the figures). Also plotted in these figures for comparison are the results presented by CMS for a model with $B R(T \rightarrow b W, t Z)=1$ (solid, black), as well as the experimental limits on the cross section, as presented in [131] and [132].

From this, the lower mass limit for the degenerate $T_{6}$ and $T_{b}^{2 / 3}$ states was determined by fitting a curve through each data set, giving limits of $364 \mathrm{GeV}$ and $413 \mathrm{GeV}$ for the isolated and non-isolated cases, respectively, from the $b W$ channel. For the $t Z$ channel, a lower mass limit of $347 \mathrm{GeV}$ and $391 \mathrm{GeV}$ is found for the same two scenarios, respectively. These mass limits correspond to a minimum value of $f$ of $892 \mathrm{GeV}$ for the isolated scenario and $621 \mathrm{GeV}$ for the non-isolated scenario, as calculated from the stronger of the discovery limits from the two decay modes. These values are in line with the theoretical assumptions of $f$ in the model, where $f$ is assumed to be of 
order $1 \mathrm{TeV}$.

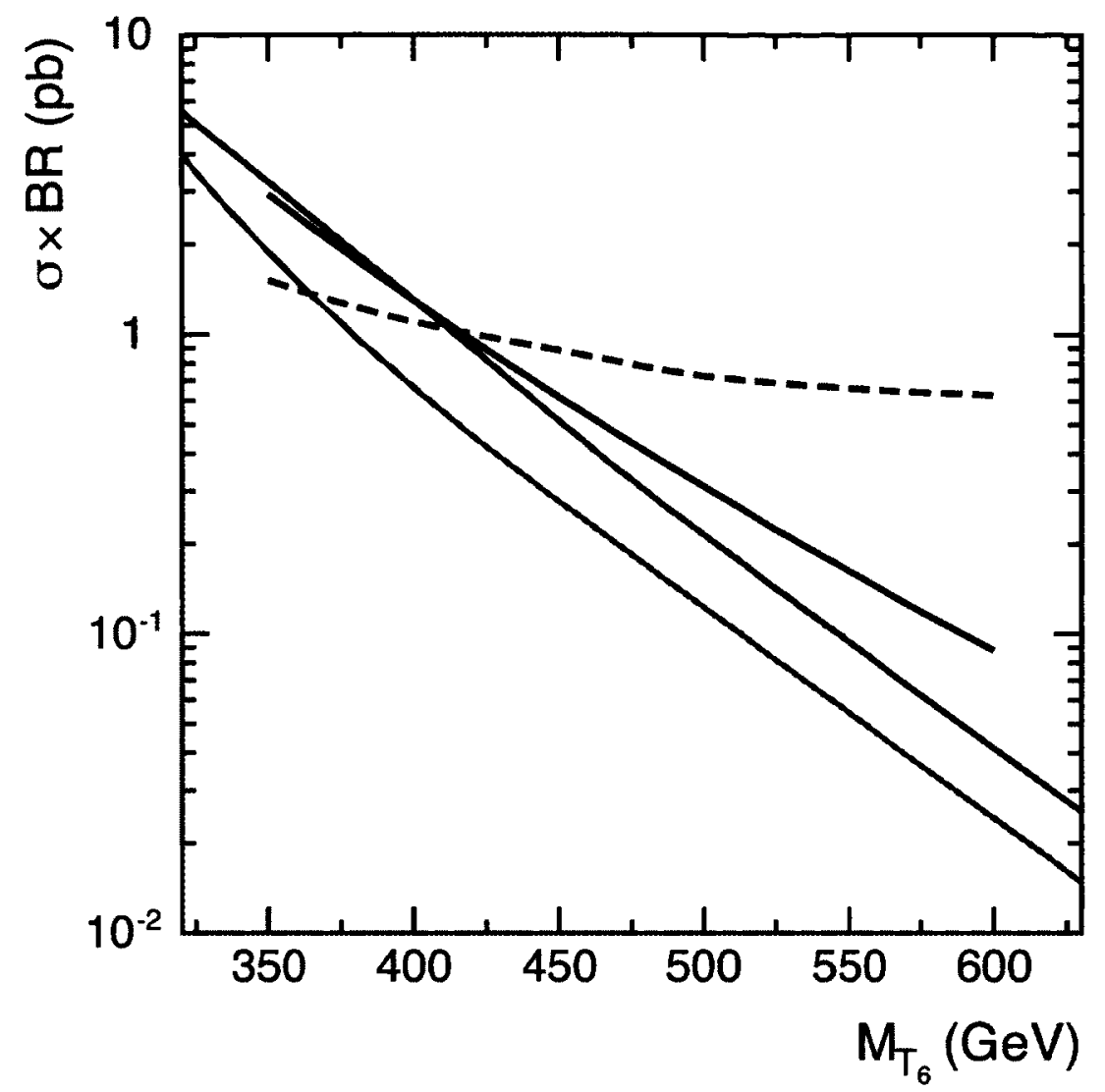

Figure 8.1: Expected cross section times branching ratio (solid) and limits (dashed) [131] in the $b W$ final state, for the isolated (light grey) and non-isolated (dark grey) scenarios. For comparison, the original heavy top-like quark pair production theoretical cross sections from [131] are also included (black), which assumes a $100 \%$ branching ratio to $b W$. 
Table 8.1: Numerical summary of heavy quark pair and single production cross sections, assuming decays to the $b W$ final state.

\begin{tabular}{|l|l|l|l|}
\hline \multicolumn{5}{|c|}{ Non-Isolated } \\
\hline$f(\mathrm{GeV})$ & $M_{T_{6}, T_{5}^{2 / 3}}(\mathrm{GeV})$ & $M_{T_{5}}(\mathrm{GeV})$ & $\sigma_{\text {pair }}(\mathrm{pb})$ \\
\hline 500 & 332.3 & 304.0 & 7.36 \\
600 & 398.8 & 396.5 & 1.39 \\
700 & 465.3 & 483.5 & 0.387 \\
800 & 531.8 & 567.6 & 0.127 \\
900 & 598.2 & 649.9 & 0.0427 \\
1000 & 664.7 & 730.9 & 0.0145 \\
1200 & 797.6 & 890.8 & 0.00168 \\
1400 & 930.6 & 1048.7 & 0.000276 \\
\hline \hline \multicolumn{5}{|c|}{ Isolated } \\
\hline$f(\mathrm{GeV})$ & $M_{T_{6}, T_{6}^{2 / 3}}(\mathrm{GeV})$ & $M_{T_{5}}(\mathrm{GeV})$ & $\sigma_{\text {pair }}(\mathrm{pb})$ \\
\hline 700 & 283.1 & 354.4 & 12.2 \\
800 & 323.5 & 424.8 & 3.63 \\
900 & 364.0 & 492.5 & 1.38 \\
1000 & 404.4 & 558.6 & 0.612 \\
1200 & 485.3 & 687.6 & 0.156 \\
1400 & 566.2 & 814.2 & 0.0419 \\
1600 & 647.1 & 939.3 & 0.0109 \\
1800 & 728.0 & 1063.4 & 0.00216 \\
\hline
\end{tabular}


Table 8.2: Numerical summary of heavy quark pair and single production cross sections, assuming decays to the $t Z$ final state.

\begin{tabular}{|l|l|l|l|}
\hline \multicolumn{5}{|c|}{ Non-Isolated } \\
\hline$f(\mathrm{GeV})$ & $M_{T_{6}, T_{b}^{2 / 3}}(\mathrm{GeV})$ & $M_{T_{5}}(\mathrm{GeV})$ & $\sigma_{\text {pair }}(\mathrm{pb})$ \\
\hline 400 & 265.9 & 198.5 & 0.224 \\
500 & 332.3 & 304.0 & 0.695 \\
600 & 398.8 & 396.5 & 0.354 \\
700 & 465.3 & 483.5 & 0.153 \\
800 & 531.8 & 567.6 & 0.0651 \\
900 & 598.2 & 649.9 & 0.0280 \\
1000 & 664.7 & 730.9 & 0.0121 \\
1200 & 797.6 & 890.8 & 0.00240 \\
1400 & 930.6 & 1048.7 & 0.000529 \\
\hline \hline \multicolumn{5}{|l|}{ Isolated } \\
\hline$f(\mathrm{GeV})$ & $M_{T_{6}, T_{b}^{2 / 3}}(\mathrm{GeV})$ & $M_{T_{5}}(\mathrm{GeV})$ & $\sigma_{\text {pair }}(\mathrm{pb})$ \\
\hline 600 & 242.7 & 279.4 & 0.119 \\
700 & 283.1 & 354.4 & 2.04 \\
800 & 323.5 & 424.8 & 1.37 \\
900 & 364.0 & 492.5 & 0.736 \\
1000 & 404.4 & 558.6 & 0.407 \\
1200 & 485.3 & 687.6 & 0.130 \\
1400 & 566.2 & 814.2 & 0.0448 \\
1600 & 647.1 & 939.3 & 0.0160 \\
1800 & 728.0 & 1063.4 & 0.00545 \\
\hline
\end{tabular}




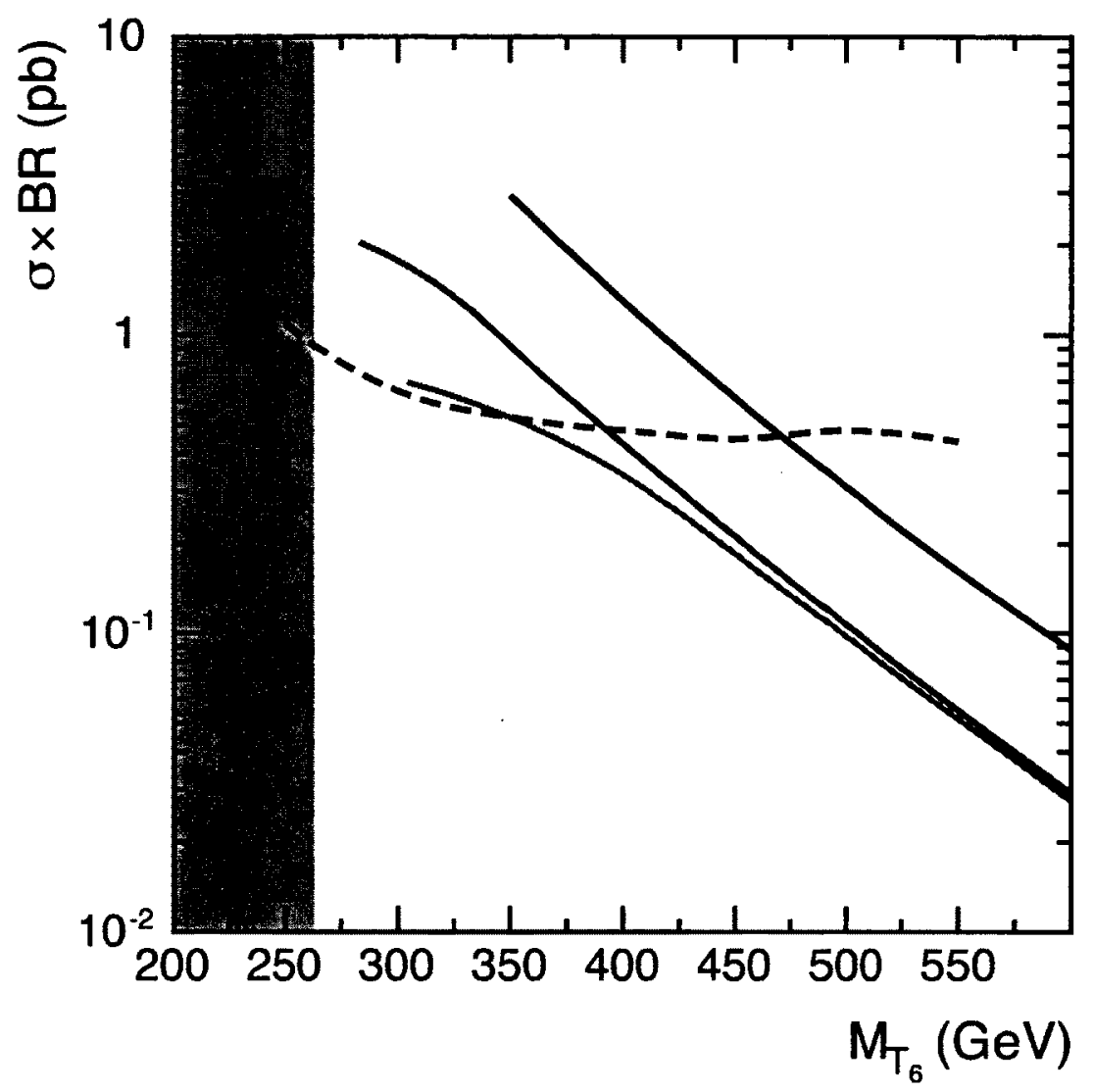

Figure 8.2: Expected cross section times branching ratio (solid) and limits (dashed) [132] in the $t Z$ final state, for the isolated (light grey) and non-isolated (dark grey) scenarios. For comparison, the original pair production theoretical cross sections from [132] are also included (black), which assumes a 100\% branching ratio to $t Z$. The light grey region represents the region of phase space where the heavy quark mass is too small to produce an on-shell $t Z$ decay $\left(M_{T_{6}}<M_{t}+M_{Z}\right)$. 


\subsection{Determining Yukawa Couplings}

For examining the potential of extracting Yukawa couplings, two different scenarios of parameter values were used, as representative of the interesting phenomenology in the model. The first parameter set is chosen where $\tan \theta_{12}>\tan \theta_{13}$, such that both the $B$ and $T_{b}^{5 / 3}$ states are observable, and their masses are measurable. The parameters for this point are: $f=700 \mathrm{GeV}, \tan \beta=\sqrt{3}, \tan \theta_{12}=1.732, \tan \theta_{13}=$ 0.7265. The second parameter set is chosen where $\tan \theta_{13}>\tan \theta_{12}$, such that the $T_{5}$ state is observable but the $B$ state is not. The parameter values for this point are: $f=936 \mathrm{GeV}, \tan \theta_{12}=0.5463$ and $\tan \theta_{13}=1.2602$.

The corresponding masses and relevant branching ratios are included in Table 8.3. In scenario 1 , both the $T$ and $T_{5}$ states are not observable in the $b W$ decay channel. This is because the branching ratio of the $T$ is too small to observe a peak above the background, and the $T_{5}$ state is too heavy and thus decays primarily through cascade decays to the $t, T, T_{6}$ and $T_{b}^{2 / 3}$ along with a neutral state $\left(Z, h\right.$ or $\left.H_{0}\right)$, or to $T_{b}^{5 / 3}$ along with a charged state $\left(W^{+}\right.$or $\left.H^{+}\right)$. The $T$ and $T_{5}$ states may be measurable in other channels, such as $t Z$, but this is beyond the scope of this analysis. To contrast this in scenario 2, the $T$ and $B$ quark cross sections are too small to observe due to the reduced phase space available to such heavy states. Additionally, the $T$ state decays primarily through cascades to other new heavy quark states, which further reduces the ability to observe a resonance in the same manner as the other heavy quarks.

\subsubsection{Heavy Top Partners}

Determining the mass of the heavy top partners is complicated by large backgrounds. Figure 8.3 shows the various contributing backgrounds to the measurement of the heavy top partner masses, assuming a $p_{T}>100 \mathrm{GeV}$ cut on the $b$-jets. The effects of the necessary cuts for identifying $b$-jets and $W$-fat-jets are incorporated into the 
Table 8.3: Masses and relevant branching ratios for all six heavy quarks in the BLH model for the two scenarios examined in this analysis.

\begin{tabular}{|l|l|l|l|}
\hline \multicolumn{4}{|c|}{ Scenario 1: $f=700 \mathrm{GeV}, \tan \theta_{12}=1.732$ and $\tan \theta_{13}=0.7265$} \\
\hline Quark & Mass & Width & Relevant BR \\
\hline$T_{6}$ & $465.3 \mathrm{GeV}$ & $2.07 \mathrm{GeV}$ & $B R\left(T_{6} \rightarrow b W^{+}\right)=49.1 \%$ \\
\hline$T_{b}^{2 / 3}$ & $465.3 \mathrm{GeV}$ & $11.57 \mathrm{GeV}$ & $B R\left(T_{b}^{2 / 3} \rightarrow b W^{+}\right)=13.2 \%$ \\
\hline$T_{b}^{5 / 3}$ & $465.3 \mathrm{GeV}$ & $6.52 \mathrm{GeV}$ & $B R\left(T_{b}^{5 / 3} \rightarrow t W^{+}\right)=99.8 \%$ \\
\hline$T$ & $483.5 \mathrm{GeV}$ & $1.81 \mathrm{GeV}$ & $B R\left(T \rightarrow b W^{+}\right)=11.7 \%$ \\
\hline$B$ & $537.3 \mathrm{GeV}$ & $4.25 \mathrm{GeV}$ & $B R\left(B \rightarrow t W^{-}\right)=98.9 \%$ \\
\hline$T_{5}$ & $807.4 \mathrm{GeV}$ & $45.73 \mathrm{GeV}$ & $B R\left(T_{5} \rightarrow b W^{+}\right) \approx 0 \%$ \\
\hline \hline Scenario $2: f=936 \mathrm{GeV}, \tan \theta_{12}=0.5463$ and tan $\theta_{13}=1.2602$ \\
\hline Quark & Mass & Width & $\operatorname{Relevant~} \mathrm{BR}$ \\
\hline$T_{6}$ & $461.5 \mathrm{GeV}$ & $7.25 \mathrm{GeV}$ & $B R\left(T_{6} \rightarrow b W^{+}\right)=58.4 \%$ \\
\hline$T_{b}^{2 / 3}$ & $461.5 \mathrm{GeV}$ & $6.52 \mathrm{GeV}$ & $B R\left(T_{b}^{2 / 3} \rightarrow b W^{+}\right)=32.6 \%$ \\
\hline$T_{b}^{5 / 3}$ & $461.5 \mathrm{GeV}$ & $2.85 \mathrm{GeV}$ & $B R\left(T_{b}^{5 / 3} \rightarrow t W^{+}\right)=99.7 \%$ \\
\hline$T$ & $971.8 \mathrm{GeV}$ & $39.90 \mathrm{GeV}$ & $B R\left(T \rightarrow b W^{+}\right)=4.25 \%$ \\
\hline$B$ & $962.6 \mathrm{GeV}$ & $26.30 \mathrm{GeV}$ & $B R\left(B \rightarrow t W^{-}\right)=2.98 \%$ \\
\hline$T_{5}$ & $547.5 \mathrm{GeV}$ & $2.51 \mathrm{GeV}$ & $B R\left(T_{5} \rightarrow b W^{+}\right)=61.1 \%$ \\
\hline
\end{tabular}


associated tagging efficiency. It is clear from this that the largest contribution to the backgrounds are the $t \bar{t}$ production and $b \bar{b} j j$ production, and thus cuts must focus on reducing these backgrounds.

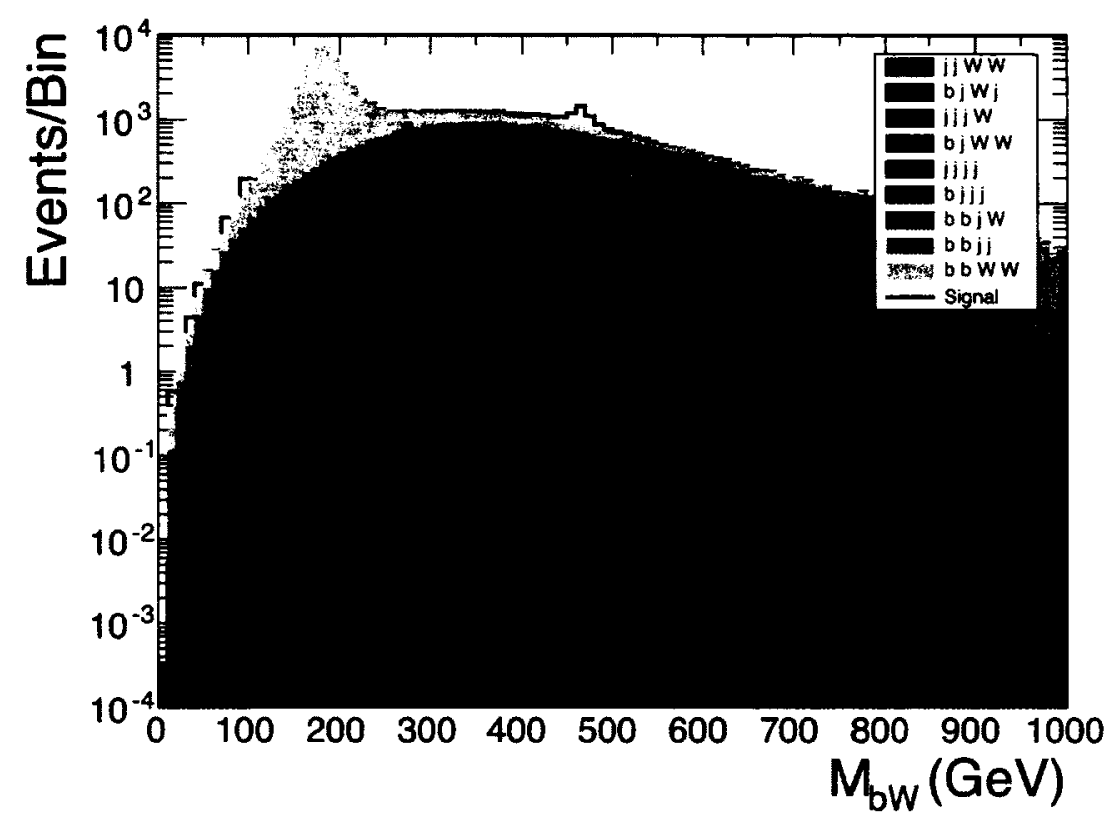

Figure 8.3: Invariant mass distribution of the $b W$ candidate pairing found from the tagging method discussed in Sec. 7.2 showing all background contributions. All distributions are presented as stacked bins. Legend is presented in inverse order. Event rate assumes an integrated luminosity of $L=100 \mathrm{fb}^{-1}$, for $14 \mathrm{TeV}$ collisions at the LHC.

While a peak from the heavy top partner is visible above the background in Fig. 8.3, the peak lacks distinguishability, as evident from the linear scale plot shown in Fig. 8.4. The signal and background events share similar kinematics, which makes cuts difficult to reduce the background more than the signal. But the signal and backgrounds do differ in terms of the $p_{T}$ distribution for the $b$-jets and the $\Delta R$ (defined as $\sqrt{\Delta \eta^{2}+\Delta \phi^{2}}$ ) separation between the centroid of the $b$ and $W$ jets. This is illustrated in Fig. 8.5, where the $p_{T}$ and $\Delta R$ phase space has been separated into bins, and the relative cross section in that bin is represented by the size of the filled square. Of note, each channel included in this pseudo-Dalitz plot is independent, and 




Figure 8.4: Invariant mass distribution of the $b W$ candidate pairing found from the tagging method discussed in Sec. 7.2, with a linear scale on the $y$-axis. All distributions are presented as stacked bins. Event rate assumes an integrated luminosity of $L=100 \mathrm{fb}^{-1}$, for $14 \mathrm{TeV}$ collisions at the LHC.

the sizes of the filled squares are not relative between each channel. This was done to increase visibility of each channel.

A cut on the $p_{T}$ of the $b$-jet candidates can be employed to significantly reduce the $b \bar{b} j j$ background with minimal effect to the signal. For a decaying top quark and for a non-resonant background such as $b \bar{b} j j$, the vast majority of the background events will result in lower $p_{T}$ for the $b$-jets than for the signal events, as the contributing diagrams favour less energetic events. While a $\Delta R$ cut also appears to be effective from Fig. 8.5, the same $t \bar{t}$ events can be reduced with less effect on the signal by instead cutting all events with $M_{b W}<300 \mathrm{GeV}$.

The final signal, after a cut of $p_{T}>150 \mathrm{GeV}$ on the $b$-jets, is shown in Fig. 8.6. While the total event rate is reduced over what is shown in Fig. 8.4, the overall signal significance is improved. By fitting an exponential function of the form $\exp \left(A_{0}+\right.$ $\left.A_{1} / M+A_{2} / M^{2}+A_{3} M+A_{4} M^{2}\right)$ to events on either side of the peak, and excluding 


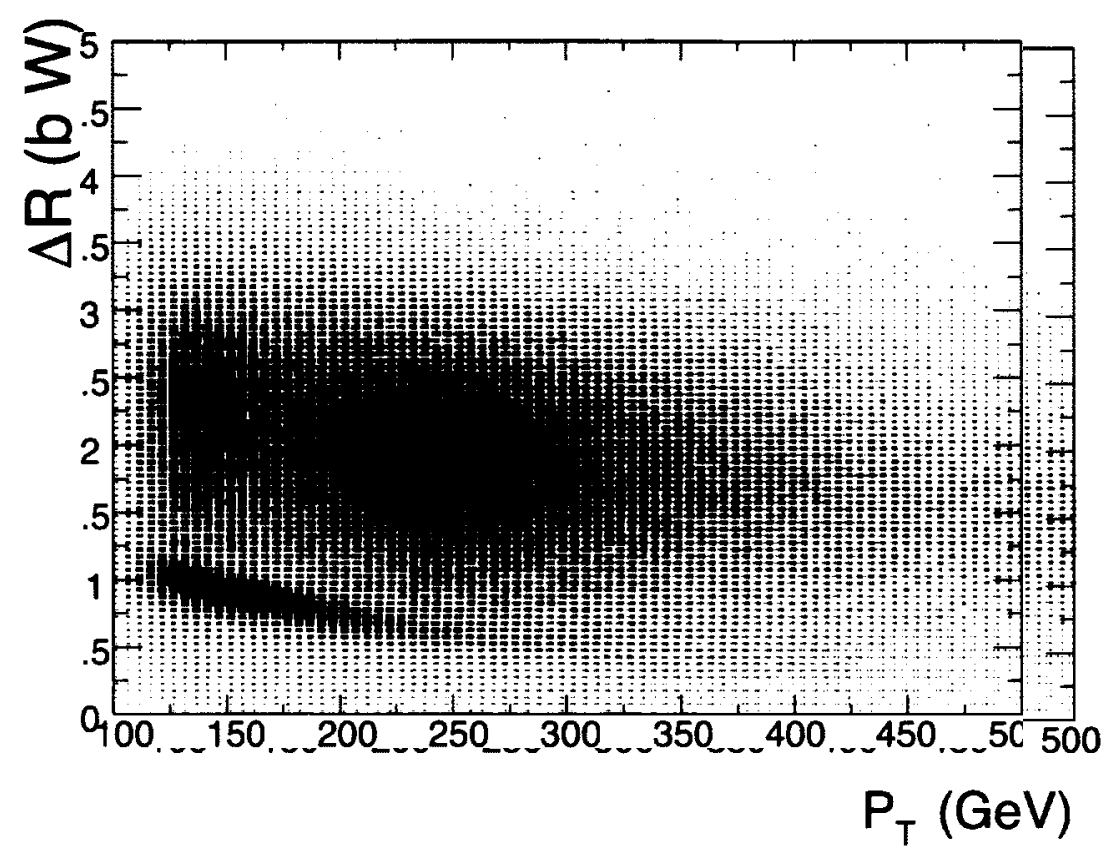

Figure 8.5: Pseudo-Dalitz plot showing regions of phase space of interest for the primary production methods that contribute to the heavy top partner signal and background. The orange bins correspond to the $b \bar{b} j j$ background, while blue bins correspond to the $t \bar{t}$ background. Black bins correspond to the signal from the heavy top partners.

all events with $M_{b W}<300 \mathrm{GeV}$, the background can be estimated and subtracted from the signal.

A fit of a Breit-Wigner function to the background-subtracted-signal is shown in Fig. 8.7. The uncertainty on the fit is purely statistical, as systematic errors require a more detailed examination of detector effects. Due to the Gaussian smearing of the hadronic events, it can be argued that the fit distribution should more appropriately be modelled as a Gaussian. However, the $\chi^{2} /$ d.o.f. for a Gaussian fit is worse than for a Breit-Wigner distribution. This may be due to effects caused by the tagging technique used to associate the "correct" pairing of $b$ and $W$ jets that are not removed with the background subtraction.

For the first scenario, the measured mass of $464.8 \pm 0.6 \mathrm{GeV}$ is within $1 \sigma$ of the input mass of $465.3 \mathrm{GeV}$, and the statistical uncertainty is comparable to the 
statistical uncertainty of current ATLAS measurements of the top quark mass using fully hadronic top decays. The width of $23.5 \pm 1.3 \mathrm{GeV}$ is dominated by the $5 \%$ energy resolution and does not represent the physical decay width of the heavy top partner, which is $2.07 \mathrm{GeV}$ for the $T_{6}$ and $11.57 \mathrm{GeV}$ for the $T_{b}^{2 / 3}$. While the $T_{b}^{2 / 3}$ width is large, the branching ratio to $b W$ is small and so it is clear that the $T_{6}$ is the dominant contribution to the peak.

In the second scenario, the measured masses are $M_{1}=462.8 \pm 0.4 \mathrm{GeV}$ and $M_{2}=548.0 \pm 0.7 \mathrm{GeV}$. The lightest state is within $3 \sigma$ of the input mass of $461.5 \mathrm{GeV}$, which is due to more events being subtracted from the low end of the distribution in the background subtraction. Even though the second mass is significantly heavier than the first, and the event rate is much lower, it is clear that the peak is measurable but should not be identified a priori as the $T_{5}$ state. As discussed previously, the $T$ and $T_{5}$ mass hierarchy depends on the mixing angles, and so it is not possible to distinguish which of these two heavy top partners is the second lightest state in the BLH model by simply observing a second mass resonance. Again, the widths are dominated by the energy resolution effects. 


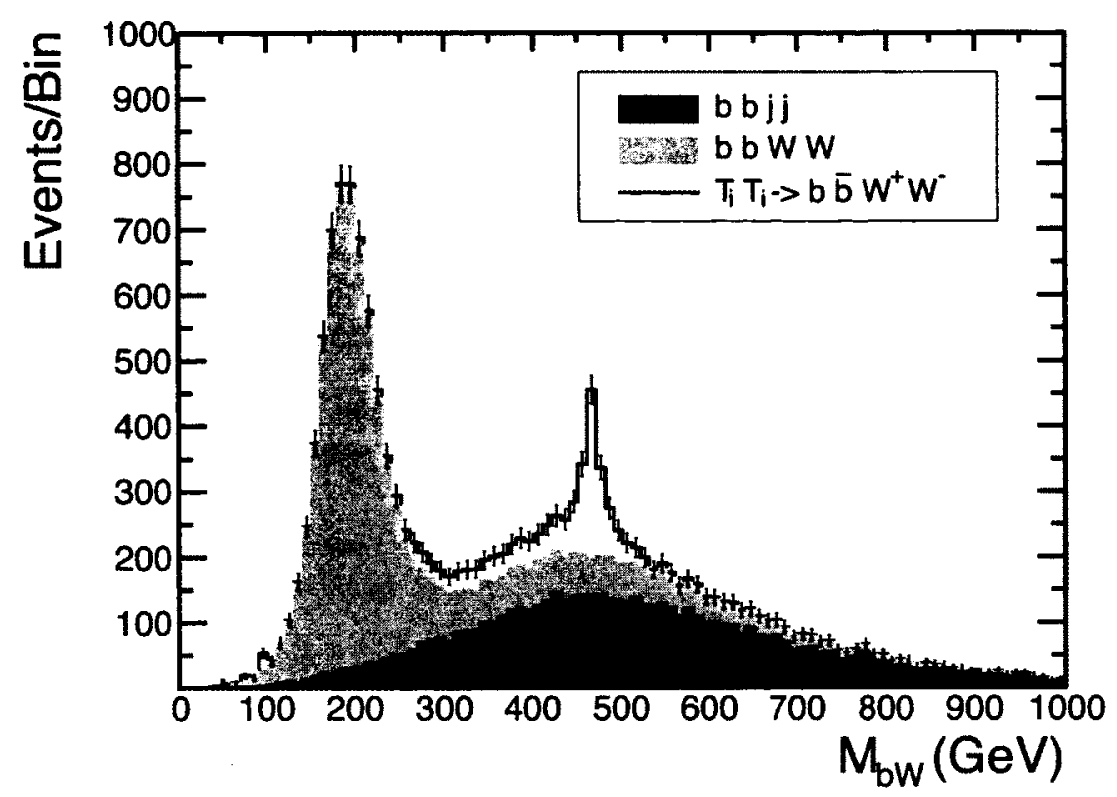

Figure 8.6: Invariant mass distribution of the $b W$ candidate pairing found from the tagging method discussed in Sec. 7.2, with a linear scale on the $y$-axis and an imposed cut on the $b$-jets of $p_{T}>150 \mathrm{GeV}$. Event rate assumes an integrated luminosity of $L=100 \mathrm{fb}^{-1}$, for $14 \mathrm{TeV}$ collisions at the LHC.

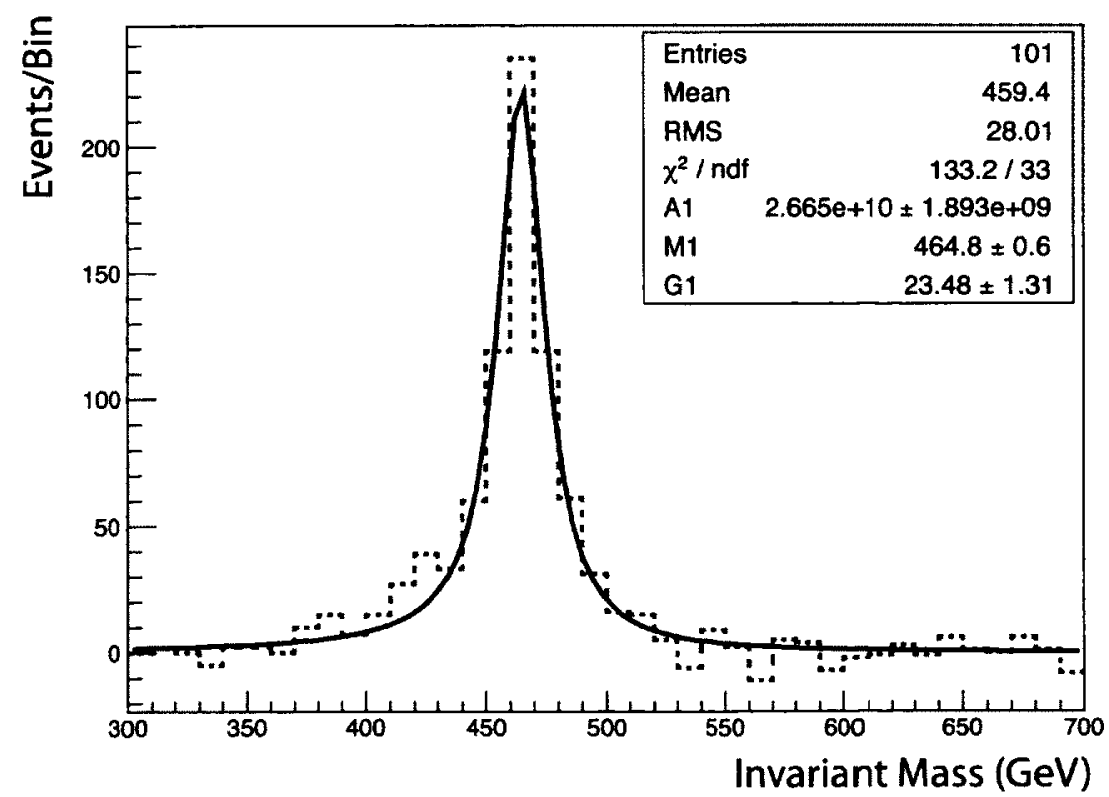

Figure 8.7: Background subtracted signal events from pair production of heavy top partners decaying to $b \bar{b} W^{+} W^{-}$final state in the first scenario, with a fit to a Breit-Wigner. 


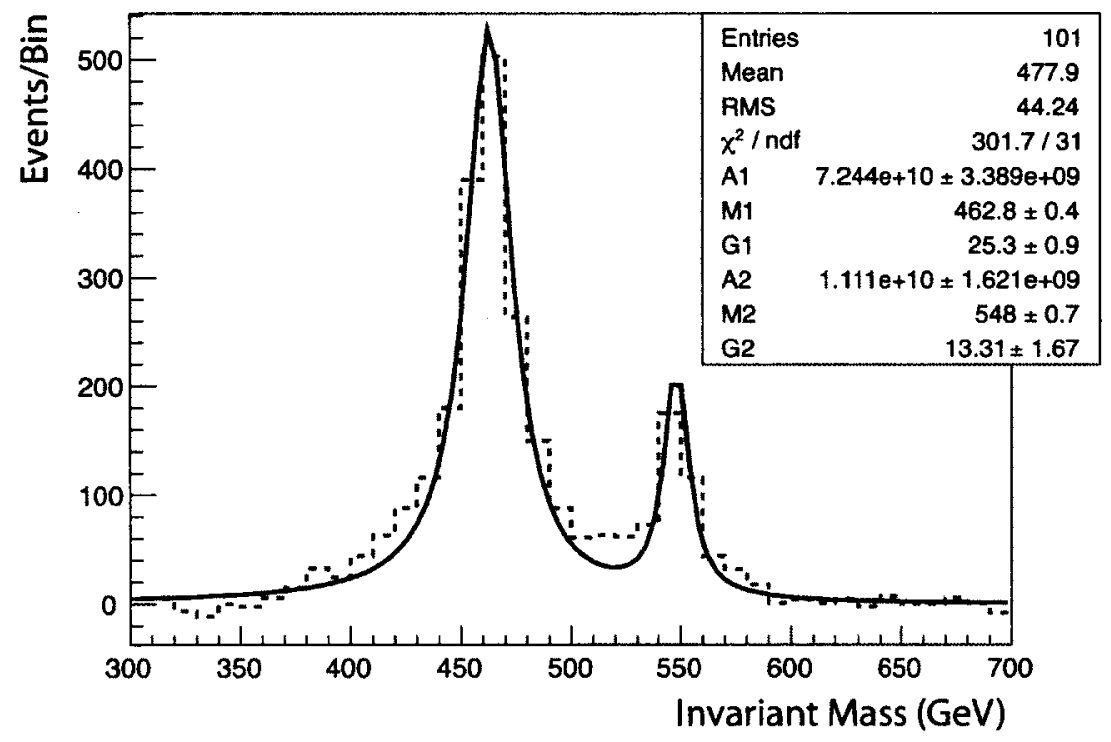

Figure 8.8: Background subtracted signal events from pair production of heavy top partners decaying to $b \bar{b} W^{+} W^{-}$final state in the second scenario, with a fit to a Breit-Wigner. 


\subsubsection{Exotic and Bottom Partner}

Estimating the backgrounds for the exotic and bottom partner quarks is more challenging than for the top partners, as all events that could fake the signal $\left(b \bar{b} W^{+} W^{+} W^{-} W^{-}\right)$necessarily require a simulation of a minimum six body final state, and events that fake $b$ and $W$ signals with light jets $(g, u, d, s, c)$ result in a large number of Feynman diagrams that are difficult to simulate. The backgrounds discussed in Sec. 7.2 are expected to be the largest contributions, and are included in this analysis. Compounding the difficulty with estimating the backgrounds, the observable event rate for this final state is reduced due to a combination of branching ratio and tagging efficiency effects. Assuming a branching ratio of $\approx 68 \%$ for the hadronic $W$ decays, a tagging efficiency of $70 \%$ for $b$-jets, and $\approx 45 \%$ for $W$-jets, the observed event rate is reduced to about $1 \%$ of the total $B \bar{B}$ and $T_{b}^{5 / 3} \bar{T}_{b}^{5 / 3}$ pair production cross section.

Reducible backgrounds without top quark intermediate states (such as the $b \bar{b}+4 j$ background) can be further reduced by requiring that the mass of both of the top quark intermediate states be approximately reconstructed. Figure 8.9 shows the distribution of the invariant mass of the intermediate $t$ state candidates for the $b \bar{b}+4 j$ background. In contrast, a real $t$ quark decaying to a $b$-jet and a $W$ produces a Breit-Wigner distribution centred at $175 \mathrm{GeV}$. By only including events where the reconstructed top quark candidate invariant mass is within $\pm 50 \mathrm{GeV}$ of the actual top quark rest mass, these kinds of backgrounds are significantly reduced as compared to the signal.

Figures 8.10 and 8.11 show the expected signal and backgrounds for the $b \bar{b} W^{+} W^{+} W^{-} W^{-}$production. While the event rate is small due to branching ratios (hadronic $W$ ) and tagging requirements, there are still several hundred useful events for each peak for determining the invariant mass of the heavy quarks in the 


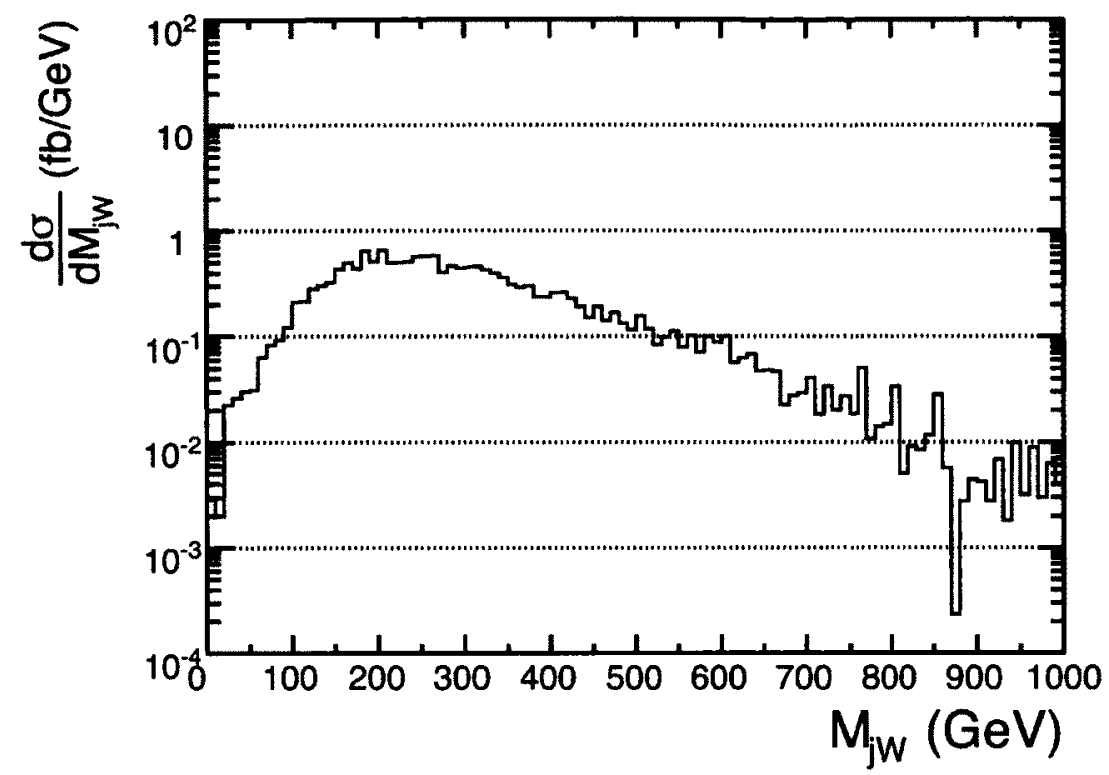

Figure 8.9: Invariant mass of the top quark candidate $(\mathrm{jW})$ intermediate state for the $b \bar{b}+4 j$ background.

first scenario. In this case, both the $T_{b}^{5 / 3}$ and the $B$ state are visible, as both have a large $(\approx 100 \%)$ branching ratio to the $t W$ final state. It is clear from this distribution that the peak cross section drops off rapidly, as both states have approximately the same branching ratio, indicating that such a measurement may not be possible for significantly heavier states.

Of note, this channel has not been examined for the second scenario, as it does not provide any additional information beyond the heavy top partner analysis.

Again, an exponential function of the form $\exp \left(A_{0}+A_{1} / M+A_{2} / M^{2}+A_{3} M+\right.$ $\left.A_{4} M^{2}\right)$ is fit to the events on either side of the central peaks, and the background events are subtracted out to fit two separate Breit-Wigner functions to the dual peaks. This is shown in Fig. 8.12. The uncertainty is purely statistical, and a larger error should be expected for an actual measurement by ATLAS or CMS due to systematics that require more detailed detector simulations to incorporate. As with the heavy top partner case, a Gaussian fit was also compared but did not represent as good a 


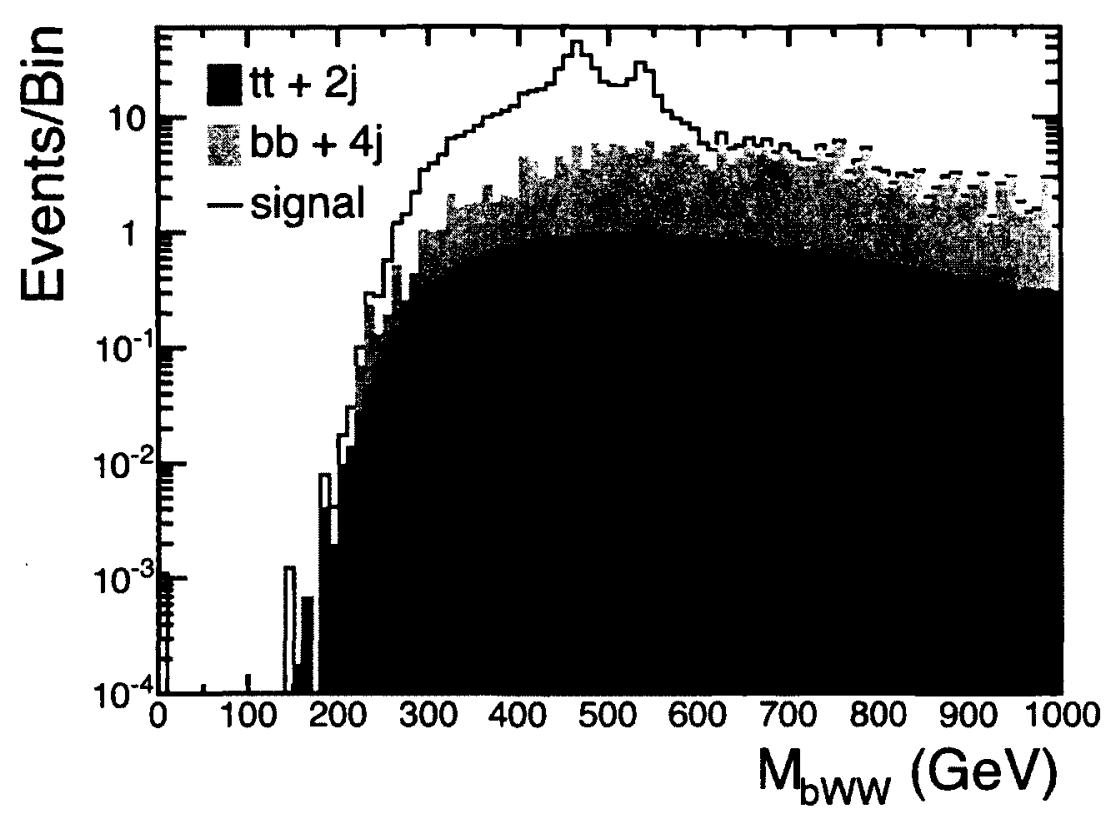

Figure 8.10: Invariant mass distribution of the reconstructed $b W W$ system for the first scenario using a log scale, assuming an integrated luminosity of $L=100 \mathrm{fb}^{-1}$, for $14 \mathrm{TeV}$ collisions at the LHC. Histograms are presented in a stacked format.

fit to the data, even though the width is not dominated by the actual decay width of the heavy quark state.

\subsubsection{Constraining Yukawa Mixing Angles}

In the first scenario, the mixing angle $\theta_{12}$ can be directly determined from the ratio of the $T_{6}$ (or $T_{b}^{5 / 3}$ ) and $B$ masses. These masses were found to be:

$$
\begin{aligned}
M_{T_{6}} & =464.8 \pm 0.6 \mathrm{GeV} \text { (input value }: 465.3 \mathrm{GeV} \text { ) } \\
M_{T_{b}^{5 / 3}} & =465.9 \pm 2.0 \mathrm{GeV} \text { (input value }: 465.3 \mathrm{GeV} \text { ) } \\
M_{B} & =537 \pm 2.7 \mathrm{GeV} \text { (input value }: 537.3 \mathrm{GeV} \text { ) }
\end{aligned}
$$

which results in an extracted/measured value of $\tan \theta_{12}=1.73 \pm 0.04$. This is in good agreement with the input value of 1.732 .

By performing a $\chi^{2}$ analysis of the measured masses against their theoretical values, scanning over $f, \tan \theta_{12}$ and $\tan \theta_{13}$, the regions of the full parameter space 


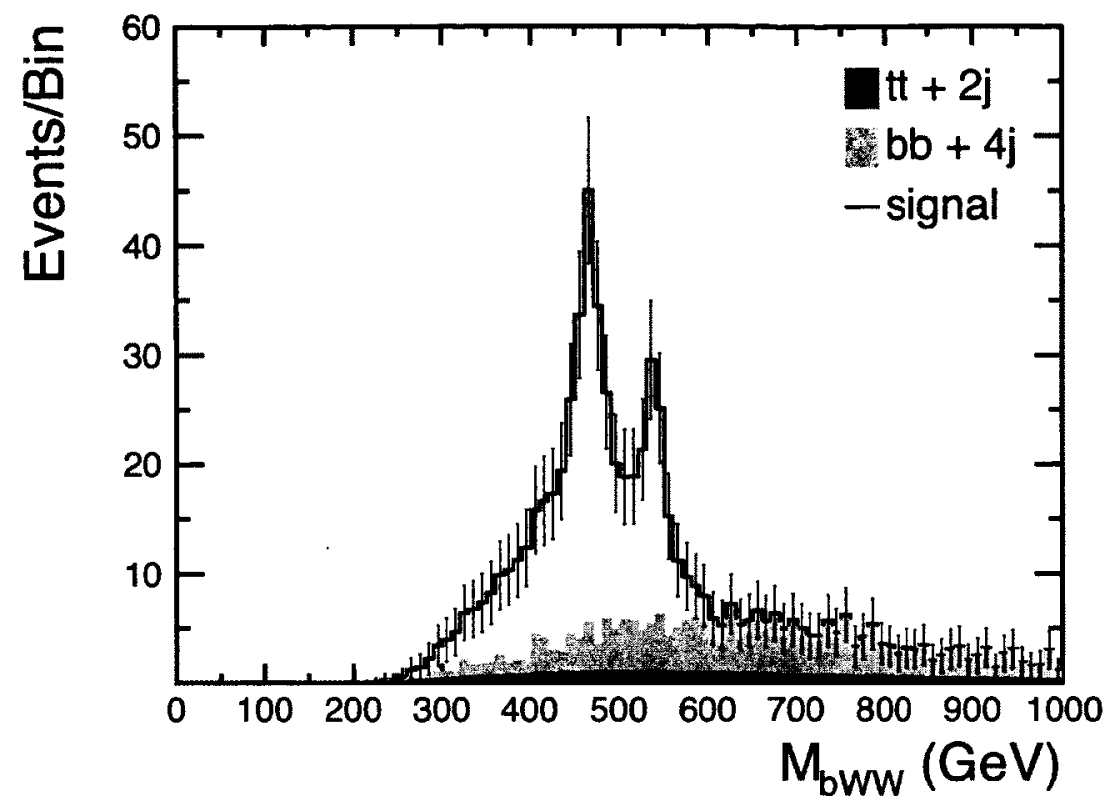

Figure 8.11: Invariant mass distribution of the reconstructed $b W W$ system for the first scenario using a linear scale, assuming an integrated luminosity of $L=100 \mathrm{fb}^{-1}$, for $14 \mathrm{TeV}$ collisions at the LHC. Histograms are presented in a stacked format.

that are allowed by these results can be explored. Using the uncertainties from the simulated values, the results of the measured masses were evaluated using:

$$
\chi^{2}=\frac{\left(M_{T_{6}}^{\text {theory }}-M_{T_{6}}^{\text {sim }}\right)^{2}}{\left(\delta M_{T_{6}}^{\text {sim }}\right)^{2}}+\frac{\left(M_{T_{b}^{5 / 3}}^{\text {theory }}-M_{T_{b}^{5 / 3}}^{\text {sim }}\right)^{2}}{\left(\delta M_{T_{b}^{5 / 3}}^{\text {sim }}\right)^{2}}+\frac{\left(M_{B}^{\text {theory }}-M_{B}^{\text {sim }}\right)^{2}}{\left(\delta M_{B}^{\text {sim }}\right)^{2}}
$$

Figure 8.13 provides a 3D $\chi^{2}$ analysis of the results, exploring the three parameters, which clearly shows that $\theta_{12}$ is strongly constrained by a measurement of the $B$ mass, while the constraints on the $f$ and $\theta_{13}$ parameters are less restrictive and highly correlated.

For the second scenario, the masses were determined to be:

$$
\begin{aligned}
& M_{T_{6}}=462.8 \pm 0.6 \mathrm{GeV} \text { (input value }: 461.5 \mathrm{GeV} \text { ) } \\
& M_{T_{5}}=548 \pm 0.7 \mathrm{GeV} \text { (input value }: 547.5 \mathrm{GeV} \text { ) }
\end{aligned}
$$

In the $\chi^{2}$ analysis shown in Fig. 8.14 , similar to the $\chi^{2}$ shown in 8.2 but for $M_{T_{6}}$ and 


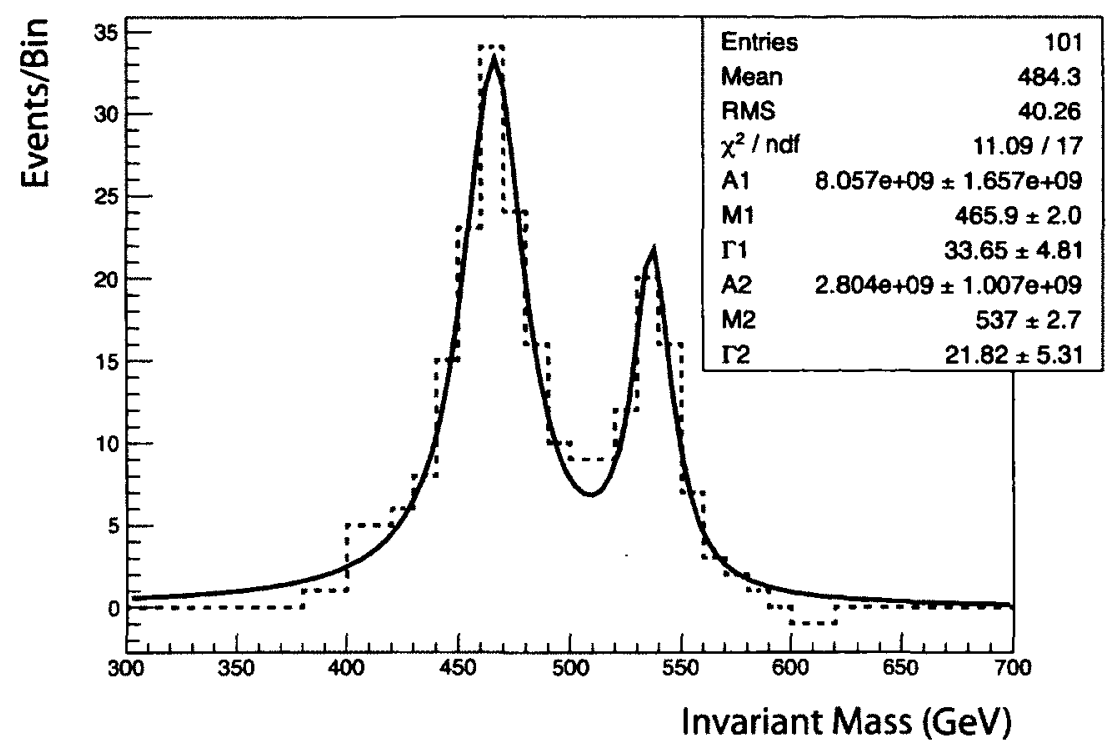

Figure 8.12: Background subtracted signal events from pair production of the heavy bottom partner and exotic quark in the first scenario, decaying to $b \bar{b} W^{+} W^{+} W^{-} W^{-}$ final state, with a fit to a dual Breit-Wigner function.

$M_{T_{5}}$, there is a mirroring along the diagonal of $\tan \theta_{12}$ and $\tan \theta_{13}$ due to the ambiguity as to whether the second lightest heavy top partner is the $T_{5}$ or the $T$ state. Assuming that the $B$ state is not observed, limits could be placed on the $B$ mass which could be used to rule out a large portion of this mirrored region. However, these limits are typically found for semi-leptonic decays due to the reduced backgrounds, and producing a reliable result requires actual data due to the importance of systematic uncertainties involved in setting limits from low statistics [137]. 

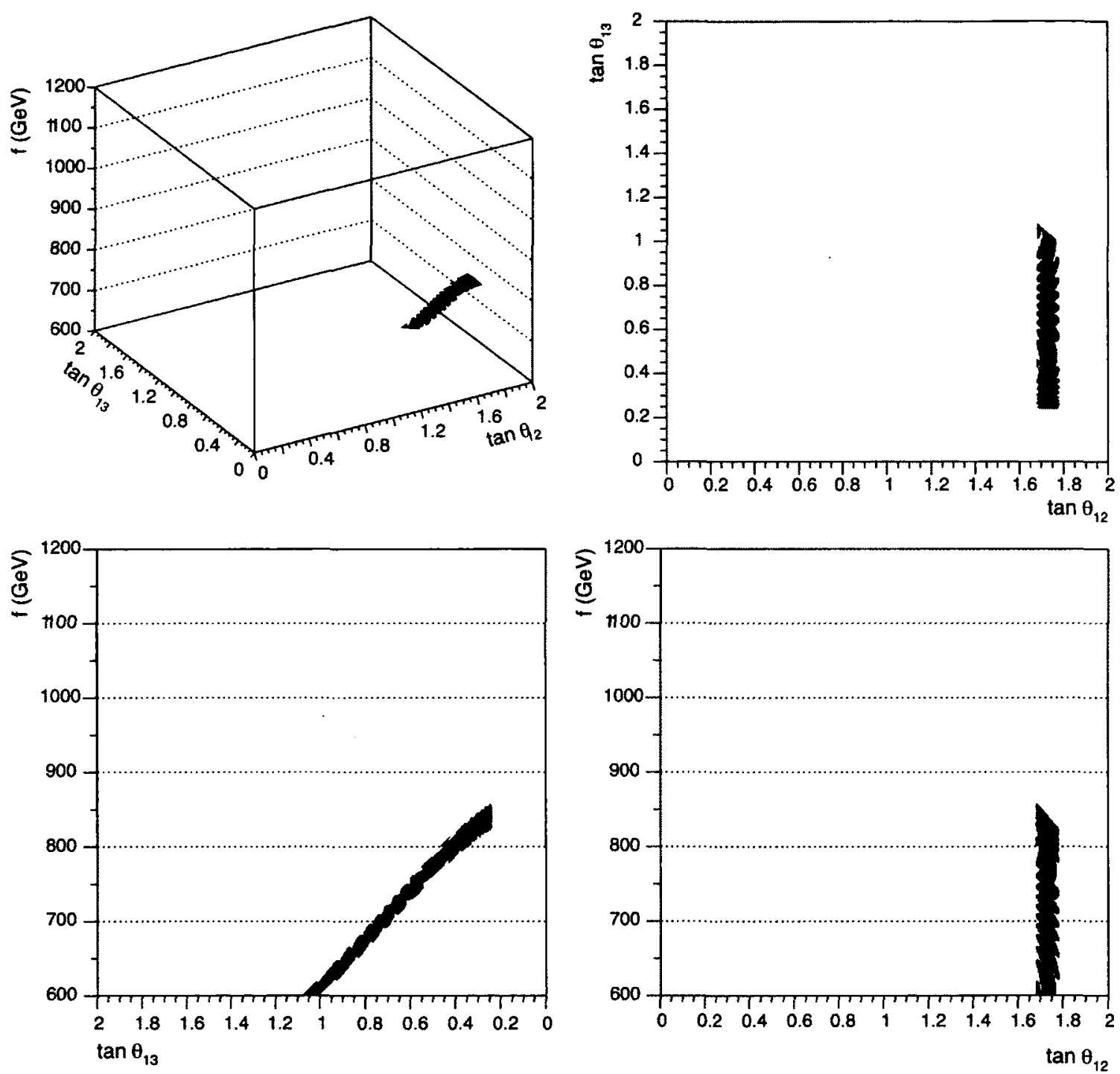

Figure 8.13: $\chi^{2}$ analysis of mass results in scenario 1, assuming purely statistical errors. Shaded region corresponds to the parameter space that could produce heavy quark masses in agreement with those observed in the simulations of scenario 1 . The $3 \mathrm{D}$ projection is accompanied by projections of the side and top view, as indicated by the axis labels (top - upper right, left - bottom left, right - bottom right). 

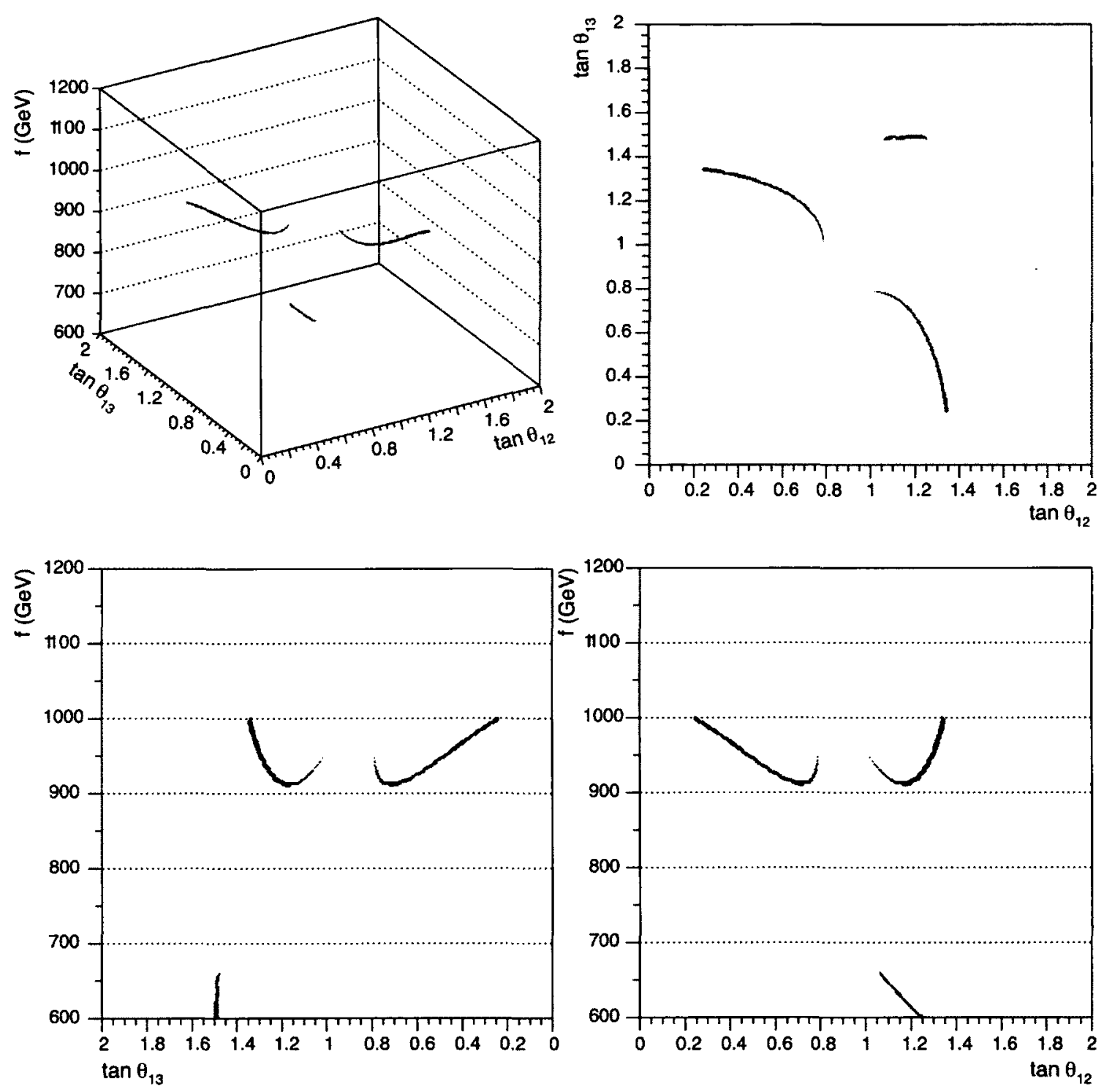

Figure 8.14: $\chi^{2}$ analysis of mass results in scenario 2, assuming purely statistical errors. Shaded region corresponds to the parameter space that could produce heavy quark masses in agreement with those observed in the simulations of scenario 2 . The $3 \mathrm{D}$ projection is accompanied by projections of the side and top view, as indicated by the axis labels (top - upper right, left - bottom left, right - bottom right). 
In summary, these are unrealistic limits as systematic uncertainties were ignored, which are likely to dominate the measurement as they do with the measurement of the top quark mass $[136,142]$. Performing an accurate analysis of the systematics involved in this type of a measurement would require details of the uncertainties in the $b$-jet and $W$-jet energy scales, a modelling of initial and final state radiation, and an analysis of the matching between Monte-Carlo simulations and actual detector events [136].

To simply explore the effects of larger uncertainties arising from systematics on the measurement of the Yukawa mixing angles, the systematic contribution can be estimated as a percentage of the heavy quark mass based on the ATLAS measurement of the top quark mass [136], giving $\delta M_{\text {sys }} \approx 1.53 \%$. The $\chi^{2}$ analyses based on this increased uncertainty are shown in Figures 8.15 and 8.16. While the constrained regions are significantly larger, the trends shown previously still remain. 

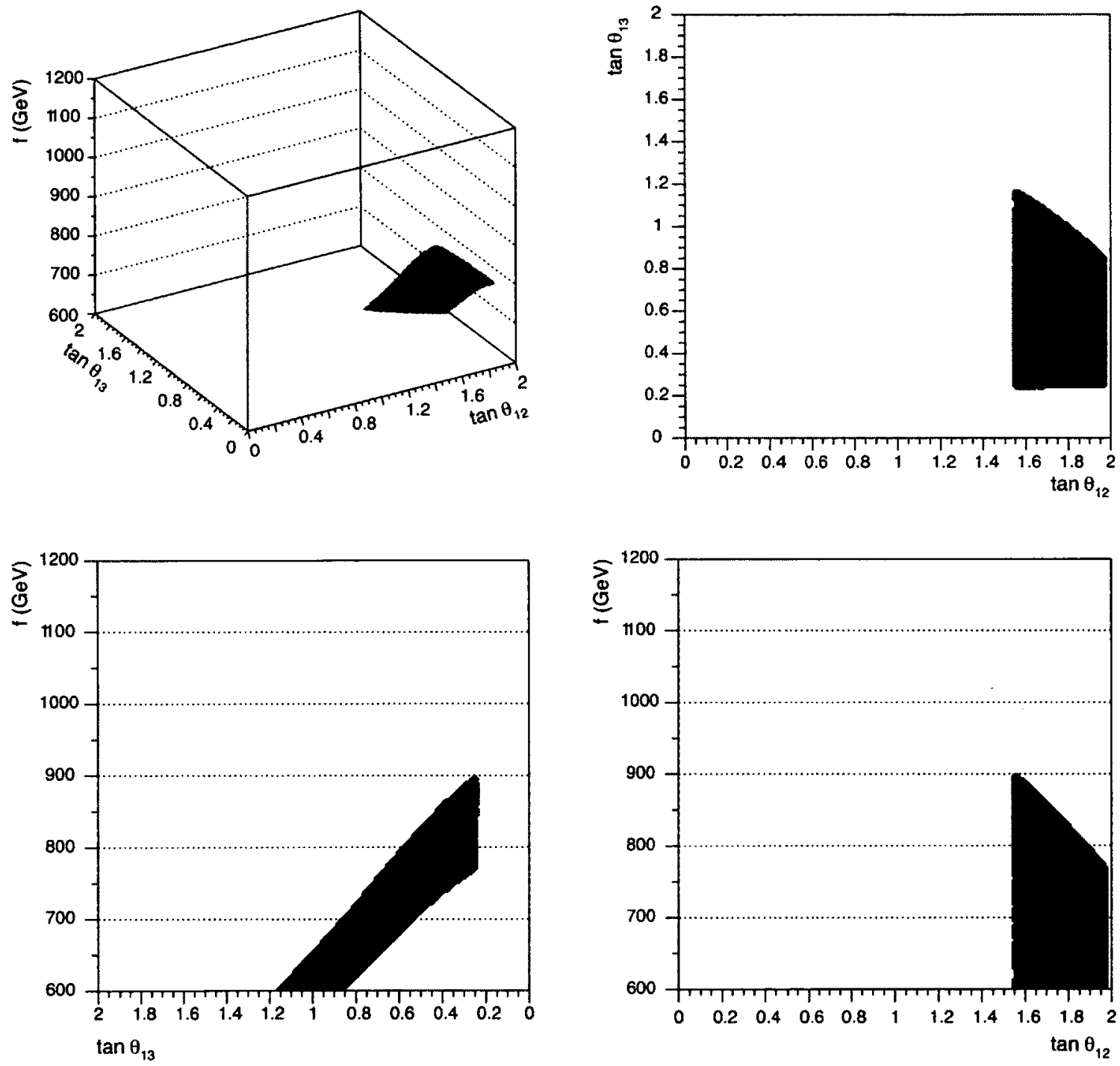

Figure 8.15: $\chi^{2}$ analysis of mass results in scenario 1, using both statistical and $1.53 \%$ systematic error. The $3 \mathrm{D}$ projection is accompanied by projections of the side and top view, as indicated by the axis labels (top - upper right, left - bottom left, right - bottom right). 

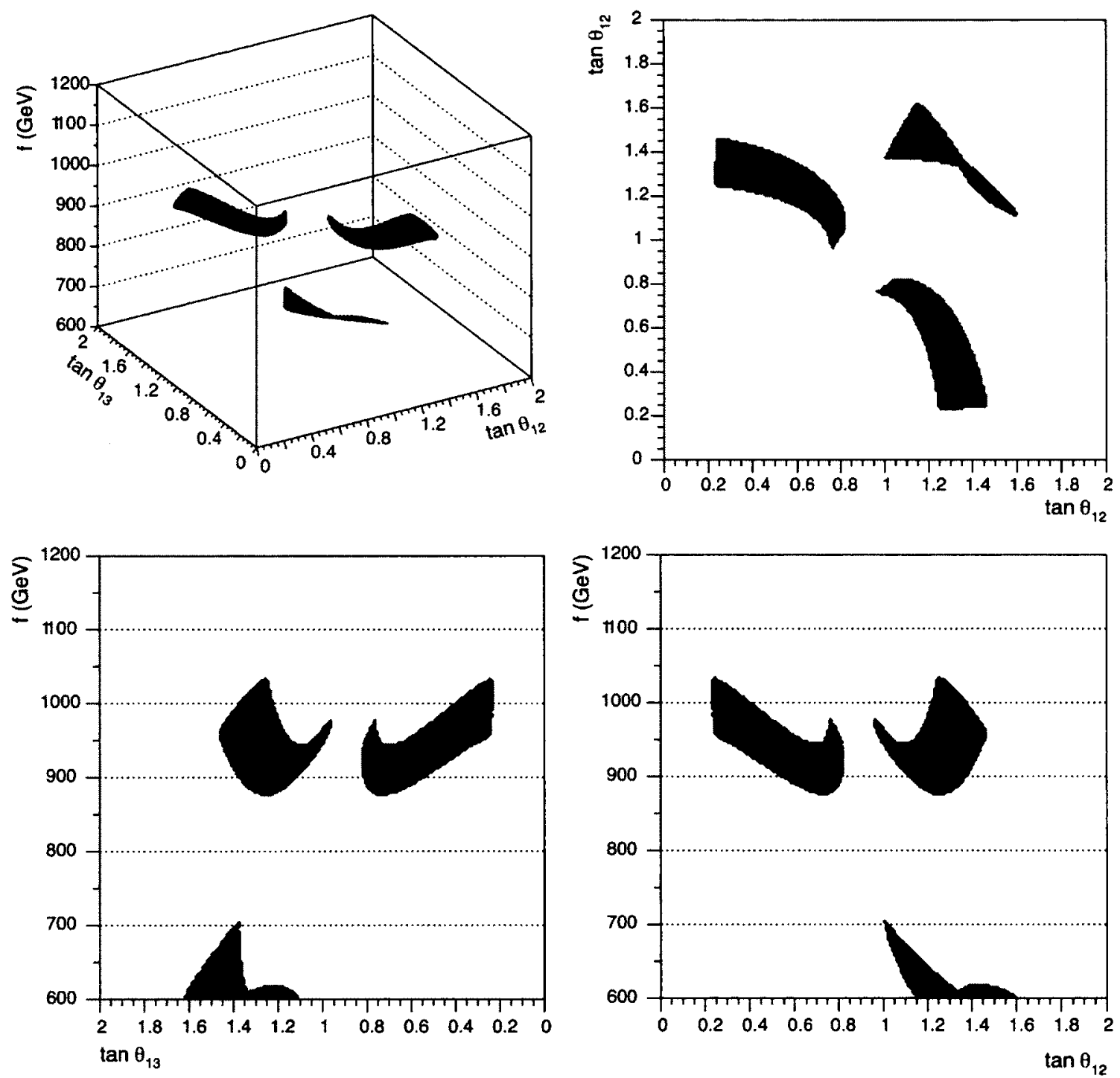

Figure 8.16: $\chi^{2}$ analysis of mass results in scenario 2, using both statistical and $1.53 \%$ systematic error. The $3 \mathrm{D}$ projection is accompanied by projections of the side and top view, as indicated by the axis labels (top - upper right, left - bottom left, right - bottom right). 


\section{Chapter 9}

\section{Summary and Conclusion}

This thesis examined the capabilities of a number of observables in establishing properties of beyond the Standard Model physics at the LHC. In Part I, several observables were defined and examined for the measurement of a single possible new state, the $Z^{\prime}$, over a wide variety of models. In Part II, the heavy quark sector of a specific model, the Bestest Little Higgs model, was examined in greater detail, as this sector provides insight into important details for resolving the hierarchy/fine-tuning problem.

\subsection{Using Pseudorapidity to Improve $Z^{\prime}$ measure- ments}

While many observables exist for exploring a new neutral gauge boson at the LHC, it is clear that a hadron collider is not able to fully examine all of the couplings of a $Z^{\prime}$. As such, there is still room to explore techniques for further understanding the experimental results from such a new particle.

I studied the use of pseudorapidity measurements in the context of re-defining and improving on several existing observables. By calculating the difference of pseudorapidity of final state fermion pairs produced from an $s$-channel resonance, it was shown that discrimination between the spin values of the intermediate boson state 
was both possible and simplified in this manner. Additionally, a comparison of the magnitudes of the pseudorapidity values for each of the pair produced fermions was shown to be an effective way to determine forward and backward tagged events in a more straightforward manner than traditional methods that reconstruct the event kinematics based on the particle four-vectors. Using pseudorapidity to measure the centre-edge asymmetry and forward-backward asymmetry in this manner has an advantage over previous approaches as these techniques rely solely on the values of the pseudorapidity - this reduces both computation time and systematic uncertainties while reproducing the results found in other analyses. In the case of the forwardbackward asymmetry, this technique is now in use by both ATLAS and CMS.

Employing the pseudorapidity method for the forward-backward asymmetry for the centroid of highly boosted $b$ - and $t$-quark jets arising from $Z^{\prime}$ decays was further studied in the context of distinguishing between models of BSM physics. Since uncertainty in the energy scale for hadronic events is large, and affects the reconstruction of the particle four vectors, the pseudorapidity technique should provide a much cleaner measurement. For a $Z^{\prime}$ of moderate mass, $A_{F B}^{q \bar{q}}$ measurements may provide some insight to distinguishing between different models. More importantly, such a measurement can contribute valuable input for measuring $Z^{\prime}$-fermion couplings in a global fit of measurements towards this goal. Unfortunately, detector resolution effects still degrade $A_{F B}$ measurements quite significantly, in particular for $t \bar{t}$ final states. Measurements using third generation fermions look promising, but it was found that a more careful detector level study is needed to properly account for resolution and other complications that arise in a real detector. 


\subsection{Distinguishing a $Z^{\prime}$ with Non-Universal Cou- plings}

In expanding upon a previous study that explored the application of third generation pair production from $Z^{\prime}$ intermediate states, I showed that such measurements were uniquely capable of providing insight into $Z^{\prime}$ models with non-universal couplings.

It should be possible to measure the decays of a moderately heavy $Z^{\prime}$ to third generation quarks, depending on the capability of the detector experiments to reject against light jet backgrounds. Such measurements would prove to be very effective at distinguishing between different models of $Z^{\prime}$, regardless of the non-universal nature of the couplings, and also give direct insight into the (non) universal nature of the $Z^{\prime}$ couplings. The measurement of the ratio of $\tau$ to $\mu$ decays of the $Z^{\prime}$, specifically, was found to be very effective in testing generation universality of the $Z^{\prime}$, and would act as a complementary study to the top and bottom ratios discussed for distinguishing between an ununified model and an extended technicolor model.

An important source of uncertainty in these measurements is the degradation of the distinguishability of the heavy fermion final state signals over the backgrounds due to detector resolution. This appears to be manageable for $b \bar{b}$ and $t \bar{t}$ final states for broad $Z^{\prime}$ resonances, such as in the Topcolor and Extended Technicolor models, but becomes increasingly important for narrow resonances such as is found in the E6 models. For $\tau$ final states, now that ATLAS and CMS have demonstrated the ability to measure $\sigma\left(p p \rightarrow Z^{0}\right) \times \mathcal{B}\left(Z^{0} \rightarrow \tau^{+} \tau^{-}\right)$the outlook is optimistic that $R_{\tau / \mu}$ can be measured well enough to distinguish between typical generation non-universal models and generation universal models. 


\subsection{Determining Yukawa Couplings in the Bestest Little Higgs Model}

Presented here was an exploration and proof of concept of the discovery search and techniques for the measurement of the mass of the heavy quarks in the Bestest Little Higgs model, and how such measurements could provide key insight into the resolution of the fine-tuning problem. It was found that only the lightest two states will likely be measurable at the $\mathrm{LHC}$, which is not sufficient to over constrain the parameter space, as desired.

Even with only $1.14 \mathrm{fb}^{-1}$ of integrated luminosity at a collision energy of $7 \mathrm{TeV}$, CMS studies have already pushed the lower mass limit of the lightest heavy top partners in the Bestest Little Higgs model above about $350 \mathrm{GeV}$, depending on the decay channel. For the regions of parameter space that were examined, the $b W$ final state was found to limit the heavy quark mass most significantly due to a larger fraction of events decaying to the $b W$ state than the $t Z$ state. The second lightest state was also included in the analysis, but it was found that the contribution of a higher mass quark resulted in very little improvement to the discovery limits due to a rapid drop off in the cross section.

The primary focus of this study was on methods for extracting the Yukawa parameters in order to establish the degree of cancellation of the divergent top quark Higgs mass correction. While next-to-leading-order (NLO) and higher order effects were not explored in this analysis, this should not significantly affect the results. Both the signal and the primary backgrounds are produced through similar QCD diagrams, and should have similar NLO factors. The overall change due to including NLO effects, then, would be to increase the overall cross sections and consequently decrease statistical uncertainties.

One of the primary challenges found in this analysis was the trade-off between 
event rates and tagging efficiencies. It may be possible to improve the tagging efficiency, at the cost of increasing the fake rate for light jet backgrounds, with a more complete understanding of the backgrounds as derived from the actual data. A further analysis by experimental groups using data driven backgrounds would be needed to explore this trade-off more thoroughly.

Due to the symmetry in the $\tan \theta_{12}-\tan \theta_{13}$ parameter space, only two heavy quark masses will likely be measurable - the lightest state $\left(T_{6}, T_{b}^{2 / 3}\right.$ and $\left.T_{b}^{5 / 3}\right)$, and either the $T_{5}$ state or the $T$ and $B$ states. When the $T_{5}$ state is light, the $T$ and $B$ states are pushed much heavier, and vice versa. As a result, two scenarios of parameter values were chosen, and picked to be representative of these two possibilities for mass hierarchies. From this, it became clear that it may only be possible to measure and analyze, at most, the two lightest heavy quark masses in the $b \bar{b} W^{+} W^{-}$and $t \bar{t} W^{+} W^{-}$ final states. However, this should still provide useful information for understanding the underlying model parameters.

In the first scenario, the ratio of the $B$ to $T_{6}$ or $T_{b}^{5 / 3}$ masses provided an effective means to determine the value of the mixing angle $\theta_{12}$. However, in this scenario, $\theta_{13}$ and $f$ were found to be more challenging to determine without additional measured masses. To determine these, the mass of the $T$ and $T_{5}$ states would also need to be found, and a $\chi^{2}$ fit involving all of the masses could be used to explore all three parameters. In the second scenario, a second peak was observed in the $b \bar{b} W^{+} W^{-}$ final state, but it was not possible to assert a priori whether this was the $T$ or the $T_{5}$ quark. This ambiguity resulted in an inability to isolate either $\theta_{12}$ or $\theta_{13}$ uniquely.

Since the parameters cannot be minimally, or (ideally) over constrained, a measurement of the single production cross section of the $T_{b}^{5 / 3}$ in association with a top quark and a jet, as shown in Fig. 9.1, would provide a measurement of the $\theta_{13}$ mixing angle. The $W t T_{b}^{5 / 3}$ vertex is dependent directly on $\theta_{13}$, as well as a number of known SM values, and the $T_{b}^{5 / 3}$ has a $100 \%$ branching ratio to the $t W$ final state in the limit 
that the mass splitting between the $t$ and $T_{b}^{5 / 3}$ is smaller than the mass of the charged scalars $(\approx 500 \mathrm{GeV})$. Thus, all other parameters in the single production cross section are well known and could be accounted for. However, this cross section is quite small, as the $W t T_{b}^{5 / 3}$ vertex is suppressed by factors of $v / f$, and would require a very high luminosity run of the LHC to measure accurately.

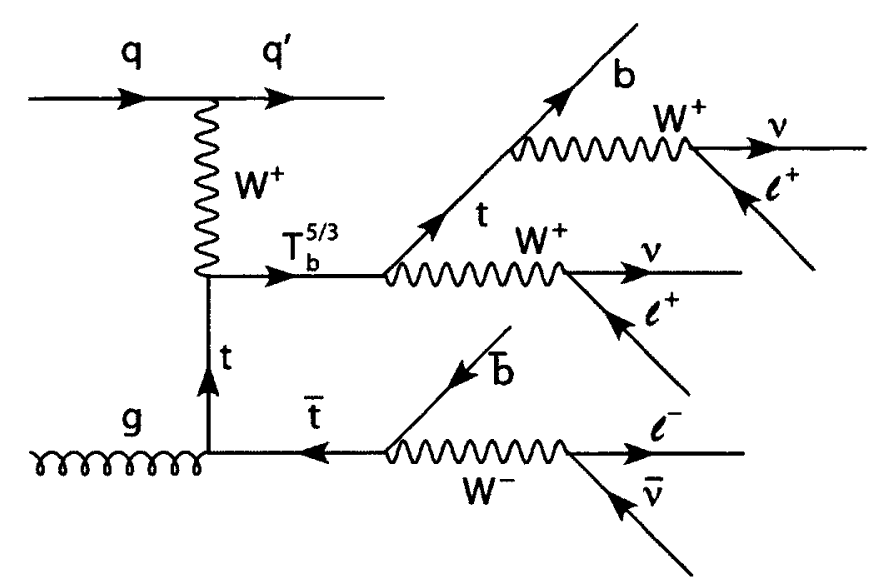

Figure 9.1: Single production mechanism of the exotic $T_{b}^{5 / 3}$ quark.

I have demonstrated the capabilities of the detector experiments at the LHC to both discover and measure the masses of the heavy quarks in a Bestest Little Higgs scenario and found that it will likely only be possible to access the lightest two masses, and thus only one of the two Yukawa mixing angles, using direct decays to SM states. Further insight into the model parameters will require either exploring cascade decays or the single production cross section of the exotic quark in the model. The importance of such measurements is clear in terms of understanding the degree of cancellation of the divergent top loop contribution to the Higgs mass, and thus resolving the biggest challenge of the fine-tuning problem. 


\section{References}

[1] R. Diener, S. Godfrey, and T. A. W. Martin, "Using Final State Pseudorapidities to Improve s-channel Resonance Observables at the LHC," Phys. Rev., vol. D80, p. 075014, 2009.

[2] R. Diener, S. Godfrey, and T. A. W. Martin, "Unravelling an Extra Neutral Gauge Boson at the LHC using Third Generation Fermions," Phys. Rev., vol. D83, p. 115008, 2011.

[3] M. Schmaltz, D. Stolarski, and J. Thaler, "The Bestest Little Higgs," JHEP, vol. 1009, p. 018, 2010.

[4] S. Godfrey, T. Gregoire, P. Kalyniak, T. A. W. Martin, and K. Moats, "Exploring the heavy quark sector of the Bestest Little Higgs model at the LHC," $J H E P$, vol. 1204, p. 032, 2012.

[5] P. Langacker, "Introduction to the Standard Model and Electroweak Physics," eprint, vol. arXiv:0901.0241 [hep-ph], pp. 3-48, 2009.

[6] Y. Fukuda et al., "Evidence for oscillation of atmospheric neutrinos," Phys. Rev. Lett., vol. 81, pp. 1562-1567, 1998.

[7] A. Bellerive, "Constraints on neutrino mixing parameters with the SNO data," eConf, vol. C030908, p. TUIT003, 2003.

[8] M. Markevitch, A. H. Gonzalez, D. Clowe, A. Vikhlinin, L. David, et al., "Direct constraints on the dark matter self-interaction cross-section from the merging galaxy cluster 1E0657-56," Astrophys. J., vol. 606, pp. 819-824, 2004.

[9] P. Langacker, "The Physics of Heavy Z' Gauge Bosons," Rev. Mod. Phys., vol. 81, pp. 1199-1228, 2009.

[10] T. G. Rizzo, " $Z$ ' phenomenology and the LHC," SLAC-PUB-12129, pp. 537$575,2006$. 
[11] A. Leike, "The Phenomenology of extra neutral gauge bosons," Phys. Rept., vol. 317, pp. 143-250, 1999.

[12] M. Cvetic and S. Godfrey, "Discovery and identification of extra gauge bosons," eprint, vol. arXiv:hep-ph/9504216, 1995.

[13] R. M. Harris, C. T. Hill, and S. J. Parke, "Cross-section for topcolor Z-prime(t) decaying to $t$ anti-t: Version 2.6," tech. rep., FERMILAB, 1999.

[14] C. T. Hill, "Topcolor: Top quark condensation in a gauge extension of the standard model," Phys. Lett., vol. B266, pp. 419-424, 1991.

[15] C. T. Hill, "Topcolor assisted technicolor," Phys. Lett., vol. B345, pp. 483-489, 1995.

[16] K. D. Lane and E. Eichten, "Natural topcolor assisted technicolor," Phys. Lett., vol. B352, pp. 382-387, 1995.

[17] K. D. Lane, "Symmetry breaking and generational mixing in topcolor assisted technicolor," Phys. Rev., vol. D54, pp. 2204-2212, 1996.

[18] K. D. Lane, "A New model of topcolor assisted technicolor," Phys. Lett., vol. B433, pp. 96-101, 1998.

[19] M. B. Popovic and E. H. Simmons, "A Heavy top quark from flavor universal colorons," Phys. Rev., vol. D58, p. 095007, 1998.

[20] W. A. Bardeen, C. T. Hill, and M. Lindner, "Minimal Dynamical Symmetry Breaking of the Standard Model," Phys. Rev., vol. D41, p. 1647, 1990.

[21] E. Malkawi, T. M. P. Tait, and C. P. Yuan, "A Model of strong flavor dynamics for the top quark," Phys. Lett., vol. B385, pp. 304-310, 1996.

[22] D. J. Muller and S. Nandi, "A Separate SU(2) for the third family: Top flavor," eprint, vol. arXiv:hep-ph/9610404, 1996.

[23] H.-J. He, T. M. P. Tait, and C. P. Yuan, "New topflavor models with seesaw mechanism," Phys. Rev., vol. D62, p. 011702, 2000.

[24] R. S. Chivukula, E. H. Simmons, and J. Terning, "A Heavy top quark and the Z b anti-b vertex in noncommuting extended technicolor," Phys. Lett., vol. B331, pp. 383-389, 1994. 
[25] R. S. Chivukula, E. H. Simmons, and J. Terning, "Limits on noncommuting extended technicolor," Phys. Rev., vol. D53, pp. 5258-5267, 1996.

[26] E. H. Simmons, "New gauge interactions and single top quark production," Phys. Rev., vol. D55, pp. 5494-5500, 1997.

[27] U. Baur and L. H. Orr, "Searching for $\mathrm{t}$ anti-t Resonances at the Large Hadron Collider," Phys. Rev., vol. D77, p. 114001, 2008.

[28] H. Georgi, E. E. Jenkins, and E. H. Simmons, "The Ununified Standard Model," Nucl. Phys., vol. B331, p. 541, 1990.

[29] R. S. Chivukula, E. H. Simmons, and J. Terning, "Limits on the ununified standard model," Phys. Lett., vol. B346, pp. 284-290, 1995.

[30] N. Arkani-Hamed, A. G. Cohen, E. Katz, A. E. Nelson, T. Gregoire, et al., "The Minimal moose for a little Higgs," JHEP, vol. 0208, p. 021, 2002.

[31] N. Arkani-Hamed, A. G. Cohen, T. Gregoire, and J. G. Wacker, "Phenomenology of electroweak symmetry breaking from theory space," JHEP, vol. 0208, p. 020, 2002.

[32] N. Arkani-Hamed, A. G. Cohen, E. Katz, and A. E. Nelson, "The Littlest Higgs," JHEP, vol. 0207, p. 034, 2002.

[33] S. L. Glashow, "Partial Symmetries of Weak Interactions," Nucl. Phys., vol. 22, pp. 579-588, 1961.

[34] S. Weinberg, "A Model of Leptons," Phys. Rev. Lett., vol. 19, pp. 1264-1266, 1967.

[35] A. Salam, Elementary Particle Theory. Stolkholm: Almquist and Wiksells; N. Svartholm edition, 1969.

[36] C. Amsler et al., "Review of Particle Physics," Phys. Lett., vol. B667, pp. 1$1340,2008$.

[37] D. Griffiths, Introduction to elementary particles. Weinheim, Germany: Wiley$\mathrm{VCH}, 2008$. 
[38] LEP and ALEPH and DELPHI and L3 and OPAL and LEP Electroweak Working Group and SLD Electroweak Group and SLD Heavy Flavor Group Collaborations, "A combination of preliminary electroweak measurements and constraints on the standard model," tech. rep., SLAC-R-701, LEPEWWG-200302, ALEPH-2003-017-PHYSICS-2003-005, DELPHI-2003-072-PHYS-937, L3NOTE-2825, OPAL-PR-392, SLD-PHYSICS-NOTE-78, 2003.

[39] The ATLAS Collaboration, "ATLAS detector and physics performance technical manual," tech. rep., CERN/LHCC/99-15, 1999.

[40] The CMS Collaboration, "CMS Physics Technical Design Report Volume I: Detector Performance and Software," tech. rep., CERN/LHCC 2006-001, 2006.

[41] R. Diener, S. Godfrey, and T. A. W. Martin, "Discovery and Identification of Extra Neutral Gauge Bosons at the LHC," eprint, vol. arXiv:0910.1334 [hep$\mathrm{ph}$, 2009.

[42] J. Kalinowski, R. Ruckl, H. Spiesberger, and P. M. Zerwas, "Supersymmetry with R-parity breaking: Contact interactions and resonance formation in leptonic processes at LEP-2," Phys. Lett., vol. B406, pp. 314-320, 1997.

[43] J. Kalinowski, R. Ruckl, H. Spiesberger, and P. M. Zerwas, "R-parity violating SUSY signals in lepton pair production at the Tevatron," Phys. Lett., vol. B414, pp. 297-304, 1997.

[44] T. Han, J. D. Lykken, and R.-J. Zhang, "On Kaluza-Klein states from large extra dimensions," Phys. Rev., vol. D59, p. 105006, 1999.

[45] J. Bijnens, P. Eerola, M. Maul, A. Mansson, and T. Sjostrand, "QCD signatures of narrow graviton resonances in hadron colliders," Phys. Lett., vol. B503, pp. 341-348, 2001.

[46] H. Davoudiasl, J. L. Hewett, and T. G. Rizzo, "Phenomenology of the RandallSundrum Gauge Hierarchy Model," Phys. Rev. Lett., vol. 84, p. 2080, 2000.

[47] F. del Aguila, M. Cvetic, and P. Langacker, "Determination of Z-prime gauge couplings to quarks and leptons at future hadron colliders," Phys. Rev, vol. D48, pp. 969-973, 1993.

[48] L. Silvestrini, "Rare decays and CP violation beyond the standard model," Int. J. Mod. Phys., vol. A21, pp. 1738-1749, 2006. 
[49] M. Cvetic and P. Langacker, "V-prime Z and V-prime W production as tests of heavy gauge boson couplings at future hadron colliders," Phys. Rev., vol. D46, p. 4943, 1992.

[50] E. Ma, "Particle Dichotomy and Left-Right Decomposition of E(6) Superstring Models," Phys. Rev., vol. D36, p. 274, 1987.

[51] S. Capstick and S. Godfrey, "A comparison of discovery limits for extra E(6) neutral gauge bosons at future colliders," Phys. Rev., vol. D37, p. 2466, 1988.

[52] S. Godfrey, J. L. Hewett, and T. G. Rizzo, "Asymmetries of heavy leptons in E(6) theories at hadron colliders," Phys. Rev., vol. D37, p. 643, 1988.

[53] J. Kang and P. Langacker, " $Z$ ' discovery limits for supersymmetric $\mathrm{E}(6)$ models," Phys. Rev., vol. D71, p. 035014, 2005.

[54] J. L. Hewett and T. G. Rizzo, "Low-Energy Phenomenology of Superstring Inspired E(6) Models," Phys. Rept., vol. 183, p. 193, 1989.

[55] D. Chang, R. N. Mohapatra, and M. K. Parida, "A New Approach to Left-Right Symmetry Breaking in Unified Gauge Theories," Phys. Rev., vol. D30, p. 1052, 1984.

[56] K. S. Babu, X.-G. He, and E. Ma, "New Supersymmetric Left-Right Gauge Model: Higgs Boson Structure and Neutral Current Analysis," Phys. Rev., vol. D36, p. 878, 1987.

[57] J. F. Gunion, A. Mendez, and F. I. Olness, "Is the left-right Higgs sector observable?," Int. J. Mod. Phys., vol. A2, p. 1085, 1987.

[58] T. G. Rizzo, "Exotic Fermion Production in a New Left-Right Model at e p Colliders," Phys. Lett., vol. B206, p. 133, 1988.

[59] F. Petriello and S. Quackenbush, "Measuring $Z^{\prime}$ couplings at the CERN LHC," Phys. Rev., vol. D77, p. 115004, 2008.

[60] S. Godfrey and T. A. W. Martin, "Identification of Extra Neutral Gauge Bosons at the LHC Using b- and t-Quarks," Phys. Rev. Lett., vol. 101, p. 151803, 2008.

[61] E. Witten, "Symmetry breaking patterns in superstring models," Nucl. Phys., vol. B258, p. 75, 1985.

[62] R. N. Mohapatra, Unification and Supersymmetry: The frontiers of quarklepton physics. New York, USA: Springer, 1986. 
[63] T. Han, H. E. Logan, and L.-T. Wang, "Smoking-gun signatures of little Higgs models," JHEP, vol. 0601, p. 099, 2006.

[64] D. E. Kaplan and M. Schmaltz, "The Little Higgs from a simple group," JHEP, vol. 0310, p. 039, 2003.

[65] W. Skiba and J. Terning, "A Simple model of two little Higgses," Phys. Rev., vol. D68, p. 075001, 2003.

[66] M. Schmaltz, "The Simplest little Higgs," JHEP, vol. 0408, p. 056, 2004.

[67] O. C. W. Kong, "Flavor and little Higgs," J. Korean Phys. Soc., vol. 45, pp. S404-S409, 2004.

[68] E. Ramirez Barreto, Y. D. A. Coutinho, and J. Sa Borges, "Extra neutral gauge boson from two versions of the 3-3-1 model in future linear colliders," Eur. Phys. J., vol. C50, pp. 909-917, 2007.

[69] A. G. Dias, J. C. Montero, and V. Pleitez, "3-3-1 models at electroweak scale," Phys. Lett., vol. B637, pp. 85-89, 2006.

[70] V. M. Abazov et al., "Search for a Narrow $t \bar{t}$ Resonance in $p \bar{p}$ Collisions at $\sqrt{s}=1.96 \mathrm{TeV}$," Phys. Rev., vol. D85, p. 051101, 2012.

[71] T. Aaltonen et al., "A Search for resonant production of $t \bar{t}$ pairs in $4.8 \mathrm{fb}^{-1}$ of integrated luminosity of $p \bar{p}$ collisions at $\sqrt{s}=1.96 \mathrm{TeV}$," Phys. Rev., vol. D84, p. $072004,2011$.

[72] J. Pumplin, D. R. Stump, J. Huston, H. L. Lai, P. M. Nadolsky, et al., "New generation of parton distributions with uncertainties from global QCD analysis," JHEP, vol. 0207, p. 012, 2002.

[73] J. Kubar-Andre and F. E. Paige, "Gluon Corrections to the Drell-Yan Model," Phys. Rev., vol. D19, p. 221, 1979.

[74] K. Melnikov and F. Petriello, "Electroweak gauge boson production at hadron colliders through O(alpha(s)**2)," Phys. Rev., vol. D74, p. 114017, 2006.

[75] C. Anastasiou, L. J. Dixon, K. Melnikov, and F. Petriello, "High precision QCD at hadron colliders: Electroweak gauge boson rapidity distributions at NNLO," Phys. Rev., vol. D69, p. 094008, 2004. 
[76] A. L. Kataev, "Higher order O (alpha**2) and O(alpha alpha-s) corrections to sigma total (e+ e- $\rightarrow$ hadron and $\mathrm{Z}$ boson decay rate," Phys. Lett., vol. B287, pp. 209-212, 1992.

[77] S. G. Gorishnii, A. L. Kataev, and S. A. Larin, "Next-To-Leading O(alpha-s**3) QCD Correction to Sigma-t (e+ e- $\rightarrow$ Hadrons): Analytical Calculation and Estimation of the Parameter Lambda (MS)," Phys. Lett., vol. B212, pp. 238$244,1988$.

[78] G. D'Agostini, W. de Boer, and G. Grindhammer, "Determiniation of alpha-s and the $\mathrm{Z} 0$ mass from measurements of the total hadronic cross-section in e+ e- annihilation," Phys. Lett., vol. B229, p. 160, 1989.

[79] U. Baur, O. Brein, W. Hollik, C. Schappacher, and D. Wackeroth, "Electroweak radiative corrections to neutral current Drell-Yan processes at hadron colliders," Phys. Rev., vol. D65, p. 033007, 2002.

[80] U. Baur, S. Keller, and W. K. Sakumoto, "QED radiative corrections to $Z$ boson production and the forward backward asymmetry at hadron colliders," Phys. Rev., vol. D57, pp. 199-215, 1998.

[81] U. Baur and D. Wackeroth, "Electroweak radiative corrections to weak boson production at hadron colliders," Nucl. Phys. Proc. Suppl., vol. 116, pp. 159-163, 2003.

[82] V. D. Barger and R. J. N. Phillips, Collider Physics. Redwood City, USA: Addison-Wesley, 1987.

[83] W. Kilian, T. Ohl, and J. Reuter, "WHIZARD: Simulating Multi-Particle Processes at LHC and ILC," Eur. Phys. J., vol. C71, p. 1742, 2011.

[84] T. Ohl, "WHiZard and O'Mega," Conference Proceedings: LoopFest V, 2006.

[85] W. Kilian, "WHIZARD manual," 2nd ECFA/DESY Study 1998-2001, vol. LCTOOL-2001-039, 2001.

[86] F. Maltoni and T. Stelzer, "MadEvent: Automatic event generation with MadGraph," JHEP, vol. 0302, p. 027, 2003.

[87] U. Baur and L. H. Orr, "High $p_{T}$ Top Quarks at the Large Hadron Collider," Phys. Rev., vol. D76, p. 094012, 2007. 
[88] T. G. Rizzo, "Distinguishing Kaluza-Klein resonances from a Z-prime in DrellYan processes at the LHC," eConf, vol. SNOWMASS-2001-P304 - C010630, p. P304, 2001.

[89] L. Randall and R. Sundrum, "A Large mass hierarchy from a small extra dimension," Phys. Rev. Lett., vol. 83, pp. 3370-3373, 1999.

[90] P. Osland, A. A. Pankov, A. V. Tsytrinov, and N. Paver, "Spin and model identification of Z' bosons at the LHC," Phys. Rev., vol. D79, p. 115021, 2009.

[91] S. Chatrchyan et al., "Measurement of the charge asymmetry in top-quark pair production in proton-proton collisions at $\sqrt{s}=7 \mathrm{TeV}$," Phys. Lett., vol. B709, pp. 28-49, 2012.

[92] G. Aad et al., "Measurement of the charge asymmetry in top quark pair production in pp collisions at $\sqrt{s}=7 \mathrm{TeV}$ using the ATLAS detector," eprint, vol. arXiv:1203.4211 [hep-ex], 2012.

[93] G. Aad et al., "The ATLAS Experiment at the CERN Large Hadron Collider," JINST, vol. 3, p. S08003, 2008.

[94] T. Aaltonen et al., "Forward-Backward Asymmetry in Top Quark Production in $p \bar{p}$ Collisions at sqrts $=1.96 \mathrm{TeV}$," Phys. Rev. Lett., vol. 101, p. 202001, 2008 .

[95] The ATLAS Collaboration, "Commissioning of the atlas high-performance btagging algorithms in the 7 tev collision data," Tech. Rep. ATLAS-CONF-2011102, CERN, Geneva, Jul 2011.

[96] A. Altheimer, S. Arora, L. Asquith, G. Brooijmans, J. Butterworth, et al., "Jet Substructure at the Tevatron and LHC: New results, new tools, new benchmarks," eprint, vol. arXiv:1201.0008 [hep-ph], 2012.

[97] D. E. Kaplan, K. Rehermann, M. D. Schwartz, and B. Tweedie, "Top Tagging: A Method for Identifying Boosted Hadronically Decaying Top Quarks," Phys. Rev. Lett., vol. 101, p. 142001, 2008.

[98] The ATLAS Collaboration, "Jet energy resolution and selection efficiency relative to track jets from in-situ techniques with the atlas detector using protonproton collisions at a center of mass energy sqrts $=7$ tev," Tech. Rep. ATLASCONF-2010-054, CERN, Geneva, Jul 2010. 
[99] G. Aad et al., "Expected Performance of the ATLAS Experiment - Detector, Trigger and Physics," eprint, vol. arXiv:0901.0512 [hep-ex], 2009.

[100] The ATLAS Collaboration, "Prospects for top anti-top resonance searches using early atlas data.," Tech. Rep. ATL-PHYS-PUB-2010-008, CERN, Geneva, Jul 2010.

[101] V. Barger, T. Han, and D. G. E. Walker, "Top Quark Pairs at High Invariant Mass: A Model-Independent Discriminator of New Physics at the LHC," Phys. Rev. Lett., vol. 100, p. 031801, 2008.

[102] K. Leney, "Vector Boson Fusion Higgs to Tau Tau Searches at the ATLAS Experiment," eprint, vol. arXiv:0810.3144 [hep-ex], 2008.

[103] A. Elagin, P. Murat, A. Pranko, and A. Safonov, "A New Mass Reconstruction Technique for Resonances Decaying to di-tau," Nucl. Instrum. Meth., vol. A654, pp. 481-489, 2011.

[104] The ATLAS Collaboration, "ATLAS: Detector and physics performance technical design report. Volume 1," tech. rep., CERN, 1999.

[105] P. Osland, A. A. Pankov, N. Paver, and A. V. Tsytrinov, "Spin identification of the Randall-Sundrum resonance in lepton-pair production at the LHC," Phys. Rev., vol. D78, p. 035008, 2008.

[106] E. W. Dvergsnes, P. Osland, A. A. Pankov, and N. Paver, "Center edge asymmetry at hadron colliders," Phys. Rev., vol. D69, p. 115001, 2004.

[107] M. Dittmar, "Neutral current interference in the TeV region: The Experimental sensitivity at the LHC," Phys. Rev., vol. D55, pp. 161-166, 1997.

[108] P. Langacker, R. W. Robinett, and J. L. Rosner, "New Heavy Gauge Bosons in p p and p anti-p Collisions," Phys. Rev., vol. D30, p. 1470, 1984.

[109] V. D. Barger, W.-Y. Keung, and E. Ma, "A gauge model with light W and Z bosons," Phys. Rev., vol. D22, p. 727, 1980.

[110] S. Godfrey, "Comparison of discovery limits for extra $\mathrm{Z}$ bosons at future colliders," Phys. Rev., vol. D51, pp. 1402-1405, 1995.

[111] V. D. Barger and T. Rizzo, "Some phenomenological aspects of the SU(2)-q x SU(2)-1 x U(1)-Y model," Phys. Rev., vol. D41, p. 946, 1990. 
[112] The ATLAS Collaboration, "Jet shapes in atlas and mc modeling," Tech. Rep. ATL-PHYS-PUB-2011-010, CERN, Geneva, Jul 2011.

[113] S. Chatrchyan et al., "Measurement of the Inclusive $\mathrm{Z}$ Cross Section via Decays to Tau Pairs in $p p$ Collisions at $\sqrt{s}=7 \mathrm{TeV}$," JHEP, vol. 1108, p. 117, 2011.

[114] S. Chatrchyan et al., "Search for Supersymmetry at the LHC in Events with Jets and Missing Transverse Energy," Phys. Rev. Lett., vol. 107, p. 221804, 2011.

[115] G. Aad et al., "Search for new phenomena in final states with large jet multiplicities and missing transverse momentum using $\sqrt{s}=7 \mathrm{TeV}$ pp collisions with the ATLAS detector," JHEP, vol. 1111, p. 099, 2011.

[116] J. A. Conley, J. S. Gainer, J. L. Hewett, M. P. Le, and T. G. Rizzo, "Supersymmetry Without Prejudice at the 7 TeV LHC," eprint, vol. arXiv:1103.1697 [hep-ph], 2011.

[117] M. Papucci, J. T. Ruderman, and A. Weiler, "Natural SUSY Endures," tech. rep., DESY, 2011.

[118] C. Csaki, J. Hubisz, G. D. Kribs, P. Meade, and J. Terning, "Big corrections from a little Higgs," Phys. Rev., vol. D67, p. 115002, 2003.

[119] C. Csaki, J. Hubisz, G. D. Kribs, P. Meade, and J. Terning, "Variations of little Higgs models and their electroweak constraints," Phys. Rev., vol. D68, p. 035009, 2003.

[120] R. Barbieri and G. F. Giudice, "Upper Bounds on Supersymmetric Particle Masses," Nucl. Phys., vol. B306, p. 63, 1988.

[121] J. A. Casas, J. R. Espinosa, and I. Hidalgo, "Implications for new physics from fine-tuning arguments. II. Little Higgs models," JHEP, vol. 0503, p. 038, 2005.

[122] G. Aad et al., "Search for dilepton resonances in pp collisions at $\sqrt{s}=7 \mathrm{TeV}$ with the ATLAS detector," Phys. Rev. Lett., vol. 107, p. 272002, 2011.

[123] M. Schmaltz and J. Thaler, "Collective Quartics and Dangerous Singlets in Little Higgs," JHEP, vol. 0903, p. 137, 2009.

[124] S. R. Coleman and E. J. Weinberg, "Radiative Corrections as the Origin of Spontaneous Symmetry Breaking," Phys. Rev., vol. D7, pp. 1888-1910, 1973. 
[125] D. Eriksson, J. Rathsman, and O. Stal, "2HDMC: Two-Higgs-Doublet Model Calculator Physics and Manual," Comput. Phys. Commun., vol. 181, pp. 189$205,2010$.

[126] E. L. Berger and Q.-H. Cao, "Next-to-Leading Order Cross Sections for New Heavy Fermion Production at Hadron Colliders," Phys. Rev., vol. D81, p. 035006, 2010.

[127] P. Meade and M. Reece, "BRIDGE: Branching ratio inquiry / decay generated events," eprint, vol. arXiv:hep-ph/0703031, 2007.

[128] J. Alwall, M. Herquet, F. Maltoni, O. Mattelaer, and T. Stelzer, "MadGraph 5 : Going Beyond," JHEP, vol. 1106, p. 128, 2011.

[129] The CMS Collaboration, "Combination of sm higgs searches," Tech. Rep. CMSPAS-HIG-11-032, CERN, Geneva, 2011.

[130] The ATLAS Collaboration, "Combination of higgs boson searches with up to $4.9 \mathrm{fb}-1$ of pp collisions data taken at a center-of-mass energy of 7 tev with the atlas experiment at the lhc," Tech. Rep. ATLAS-CONF-2011-163, CERN, Geneva, Dec 2011.

[131] S. Chatrchyan et al., "Search for heavy, top-like quark pair production in the dilepton final state in $p p$ collisions at $\sqrt{s}=7 \mathrm{TeV}$," tech. rep., CERN, 2012.

[132] S. Chatrchyan et al., "Search for a Vector-like Quark with Charge $2 / 3$ in $\mathrm{t}+\mathrm{Z}$ Events from pp Collisions at $\sqrt{s}=7 \mathrm{TeV}$," Phys. Rev. Lett., vol. 107, p. 271802, 2011.

[133] D. Stump, J. Huston, J. Pumplin, W.-K. Tung, H. L. Lai, et al., "Inclusive jet production, parton distributions, and the search for new physics," JHEP, vol. 0310, p. 046, 2003.

[134] S. P. Martin, "A Supersymmetry primer," eprint, vol. hep-ph/9709356, 1997.

[135] N. Arkani-Hamed, A. G. Cohen, and H. Georgi, "Electroweak symmetry breaking from dimensional deconstruction," Phys. Lett., vol. B513, pp. 232-240, 2001.

[136] The ATLAS Collaboration, "Measurement of the top quark mass from 2011 atlas data using the template method," Tech. Rep. ATLAS-CONF-2011-120, CERN, Geneva, Aug 2011. 
[137] G. Aad et al., "Search for down-type fourth generation quarks with the ATLAS detector in events with one lepton and high transverse momentum hadronically decaying W bosons in $\sqrt{s}=7 \mathrm{TeV}$ pp collisions," eprint, vol. arXiv:1202.6540 [hep-ex], 2012.

[138] M. L. Mangano, M. Moretti, F. Piccinini, R. Pittau, and A. D. Polosa, "ALPGEN, a generator for hard multiparton processes in hadronic collisions," JHEP, vol. 0307, p. $001,2003$.

[139] F. Caravaglios, M. L. Mangano, M. Moretti, and R. Pittau, "A New approach to multijet calculations in hadron collisions," Nucl. Phys., vol. B539, pp. 215-232, 1999.

[140] M. L. Mangano, M. Moretti, and R. Pittau, "Multijet matrix elements and shower evolution in hadronic collisions: $W b \bar{b}+n$ jets as a case study," Nucl. Phys., vol. B632, pp. 343-362, 2002.

[141] The ATLAS Collaboration, "Data-driven determinations of $\mathrm{w}, \mathrm{z}$ and top backgrounds to supersymmetry," Tech. Rep. ATL-PHYS-PUB-2009-064. ATLCOM-PHYS-2009-187, CERN, Geneva, Dec 2008.

[142] T. D. C. The CDF Collaboration, "Combination of CDF and D0 results on the mass of the top quark using up to $5.8 \mathrm{fb}-1$ of data," tech. rep., FERMILAB, 2011.

[143] S. Nie and M. Sher, "Vacuum stability bounds in the two Higgs doublet model," Phys. Lett., vol. B449, pp. 89-92, 1999.

[144] S. Kanemura, T. Kasai, and Y. Okada, "Mass bounds of the lightest CP even Higgs boson in the two Higgs doublet model," Phys. Lett., vol. B471, pp. 182$190,1999$. 


\section{Appendix A}

\section{Neutral Resonance Model Details}

\section{A.1 R-Parity Violating $\tilde{\nu}$ (spin-0)}

Many supersymmetric theories include a $Z_{2}$ symmetry known as $R$-parity, where supersymmetric partners of the SM fields are charged with odd $R$-parity. This prevents decays of supersymmetric particles to SM particle pairs, and sets a dark matter candidate as the lightest supersymmetric partner.

For models with $R$-parity breaking processes $[42,43]$, lepton pairs can be produced in the $S$-channel production and decay of a sneutrino $(\tilde{\nu})$, in addition to the SM electroweak production mechanisms. At the parton level, the combined BSM $(\tilde{\nu})$ and $\mathrm{SM}(\gamma, Z)$ cross section appears as:

$$
\frac{d \hat{\sigma}}{d \cos \hat{\theta}}\left(q \bar{q} \rightarrow \ell^{+} \ell^{-}\right)=\frac{d \hat{\sigma}_{\bar{\nu}}}{d \cos \hat{\theta}}+\frac{d \hat{\sigma}_{\gamma, Z}}{d \cos \hat{\theta}} .
$$

Due to details of the model, the $\tilde{\nu}$ mediates $d \bar{d}$ annihilation only, with an angular distribution of:

$$
\frac{d \hat{\sigma}_{\tilde{\nu}}}{d \cos \hat{\theta}}=\frac{1}{3} \frac{\pi \alpha^{2} \hat{s}}{4} \frac{\left(\lambda_{131} \lambda_{311}^{\prime} / e^{2}\right)^{2}}{\left(\hat{s}-m_{\tilde{\nu}}^{2}\right)^{2}+\Gamma_{\tilde{\nu}}^{2} m_{\tilde{\nu}}^{2}},
$$

where $\sqrt{\hat{s}}$ is the centre-of-mass energy in the $q \bar{q}$ system. The $\lambda$ parameters are the couplings in the model, which are taken to be $\lambda \lambda^{\prime}=0.05^{2}$. 


\section{A.2 Randall-Sundrum Graviton (spin-2)}

A distinctive signature of many models of extra dimensions are Kaluza-Klein or Randall-Sundrum gravitons that decay to lepton pairs [44-46,88]. Since gravitons are spin-2, they produce a unique angular distribution that should be distinguishable at the LHC. The combined parton level BSM $\left(\hat{\sigma}_{q \bar{q}}^{G}\right.$ and $\left.\hat{\sigma}_{g g}^{G}\right)$ and $\operatorname{SM}(\gamma, Z)$ cross section is given by:

$$
\frac{d \hat{\sigma}}{d \cos \hat{\theta}}\left(q \bar{q} \rightarrow \ell^{+} \ell^{-}\right)=\frac{d \hat{\sigma}_{q \bar{q}}^{G}}{d \cos \hat{\theta}}+\frac{d \hat{\sigma}_{g g}^{G}}{d \cos \hat{\theta}}+\frac{d \hat{\sigma}_{\gamma, Z}}{d \cos \hat{\theta}} .
$$

The angular distribution of the graviton cross section is separated into two parts production from $q \bar{q}$ annihilations and production from gluon fusion, $g g$. In general, the energy dependence of these angular distributions are identical, and given by:

$$
\frac{d \hat{\sigma}_{i}^{G}}{d \cos \hat{\theta}}=\frac{\kappa^{4} M^{2}}{640 \pi^{2}}\left|\frac{M^{2}}{M^{2}-M_{G}^{2}+i M_{G} \Gamma_{G}}\right|^{2} \Delta_{i}(\cos \hat{\theta}) .
$$

In this form, $\kappa=\sqrt{2} \frac{x_{1}}{M_{G}} c$, where $x_{1}$ is the first root of the Bessel function $J_{1}\left(x_{1}=\right.$ $3.8317)$ and $c$ is a constant in the model with a limit of $c \leq 0.1$. The subscript $i$ refers to either the $g g$ or $q \bar{q}$ angular distributions, which are given by:

$$
\begin{array}{r}
\Delta_{q \bar{q}}=\frac{5 \pi}{64 N_{c}}\left(1-3 \cos ^{2} \hat{\theta}+4 \cos ^{4} \hat{\theta}\right) \\
\Delta_{g g}=\frac{5 \pi}{16\left(N_{c}^{2}-1\right)}\left(1-\cos ^{4} \hat{\theta}\right)
\end{array}
$$

\section{A.3 Other $Z^{\prime}$ Models}

\section{A.3.1 E6 Models (E6)}

In the $E_{6}$ models $[54,61]$, the $E_{6}$ group breaks either directly to an $S O(10) \times U(1)_{\psi}$ or along the path $S O(10) \rightarrow S U(5) \times U(1)_{\chi}$, resulting in new gauge bosons associated with the $U(1)_{\psi}$ or $U(1)_{\chi}$ symmetries. A more general gauge boson can arise as a 
mixing between these two scenarios, parameterized by a mixing angle, $\theta_{E_{6}}$. In this general case, the charge of a fermion is given by $Q_{E_{6}}\left(\theta_{E_{6}}\right)=\cos \theta_{E_{6}} Q_{\chi}+\sin \theta_{E_{6}} Q_{\psi}$.

The charges for the $E_{6}$ models are listed in Table A.1. In the conjugate notation, a right handed SM fermion will have the negative of the corresponding charge for a conjugate field (i.e. $Q_{u_{R}}=-Q_{u^{c}}$ ). In the notation of the $Z^{\prime}$ model couplings described in Chapter 4 , the couplings $L_{f}$ and $R_{f}$ are then given by $L_{f}=Q_{E_{6}}(f)$ and $R_{f}=-Q_{E_{6}}\left(f^{c}\right)$.

The coupling strength for the $E_{6}$ models is a free parameter, with the restriction $g_{Z^{\prime}} / g_{Z} \leq \sqrt{\frac{5}{3} \mathrm{~s}_{W}^{2}}[12]$. For all calculations, I use the maximum value, $g_{Z^{\prime}} / g_{Z}=\sqrt{\frac{5}{3} \mathrm{~s}_{W}^{2}}$, as was done by Cvetič and Godfrey [12].

Table A.1: Couplings for the $E_{6} \psi$ and $\chi$ models. Couplings to right handed fermions are the negative of the left handed conjugates. These groups allow for several new fermions, including: $D$, a down type quark that transforms as a singlet under $S U(2)_{L},\left(E^{0}, E^{-}\right)$, forming a lepton doublet that transforms as a vector under $S U(2)_{L}$, and $N$ and $S$, massive Weyl neutrinos.

\begin{tabular}{|c|c||c|c|c|}
\hline$S O(10)$ Rep. & $S U(5)$ Rep. & Particle Designation & $2 \sqrt{6} Q_{\psi}$ & $2 \sqrt{10} Q_{\chi}$ \\
\hline \hline \multirow{2}{*}{16} & 10 & $\left(u, d, u^{c}, e^{c}\right)$ & -1 & 1 \\
& $5^{*}$ & $\left(d^{c}, \nu, e^{-}\right)$ & 3 & 1 \\
& 1 & $N^{c}$ & -5 & 1 \\
\hline \multirow{2}{*}{10} & 5 & $\left(D,\left(E^{0}\right)^{c}, E^{+}\right)$ & 2 & -2 \\
& $5^{*}$ & $\left(D^{c}, E^{0}, E^{-}\right)$ & -2 & -2 \\
\hline 1 & 1 & $S^{0}$ & 0 & 4 \\
\hline
\end{tabular}




\section{A.3.2 Left-Right Symmetric Models (LR \& ALR)}

In the Left-Right Symmetric Models [12,62] (LR and ALR), the higher group $S O(10)$ (or even the $E_{6}$ group) follows the breaking scheme $S O(10) \rightarrow S U(3)_{c} \times S U(2)_{L} \times$ $S U(2)_{R} \times U(1)_{B-L}$. In general, two isospin assignments are possible for the $S U(2)_{R}$ subgroup, leading to the Left-Right (LR) Symmetric model and the Alternate LeftRight (ALR) Symmetric model. [50,56-58]

The couplings of the fermions under the new gauge group are then given by the following relation, for both the LR and ALR models:

$$
\begin{aligned}
& L_{f}=\mathrm{s}_{W}^{2}\left(T_{3 L}-Q_{e m}\right) \\
& R_{f}=\kappa^{2}\left(1-\mathrm{s}_{W}^{2}\right) T_{3 R}-s_{W}^{2} Q_{e m} .
\end{aligned}
$$

Constraints on the mixing between the $S U(2)_{R}$ and $S U(2)_{L}$ gauge coupling constant require that $0.55 \leq \kappa^{2} \equiv\left(\frac{g_{R}}{g_{L}}\right)^{2} \leq 1(2)$ (value of 1 or 2 depends on whether the symmetry is broken by a Higgs doublet or triplet). The isospin assignments, $T_{3}$, are the same as in the SM for $T_{3 L}$. In the LR model, the doublets $\left(u_{R}, d_{R}\right)$ and $\left(\nu_{R}, e_{R}\right)$ have isospin assignments of $(1 / 2,-1 / 2)$, similar to the left handed doublets in the SM. In the ALR model, right handed up type quarks have a hypercharge assignment of $+1 / 2$, while down type quarks are singlets under $S U(2)_{R}$, and the lepton doublet is inverted $\left(\left(e_{R}, \nu_{R}\right) \rightarrow(1 / 2,-1 / 2)\right)$.

The coupling strengths for both models are identical, and related to the SM $Z^{0}$ coupling strength via the relation:

$$
\frac{g_{Z^{\prime}}}{g_{Z}}=\frac{1}{\sqrt{\kappa^{2}-\left(1+\kappa^{2}\right) \mathrm{s}_{W}^{2}}}
$$

Unless otherwise stated, $\kappa=1$ is used for calculations. 


\section{A.3.3 Little Higgs Models}

The Little Higgs Models [30-32] are designed as possible solutions to the hierarchy problem by introducing the Higgs boson as a pseudo-Nambu-Goldstone boson that is broken at the TeV scale to the SM Higgs doublet. These models feature an expanded gauge group, which typically includes a new, heavy neutral gauge boson. A number of Little Higgs models have been created, and three Little Higgs models are included in this study.

\section{Littlest Higgs Model (LH)}

The Littlest Higgs Model (LHM) [32,63] is a variant of the Little Higgs models in which the $\mathrm{SM} S U(2)_{L} \times U(1)_{Y}$ results from the breaking of a gauged $[S U(2) \times U(1)]^{2}$ product group that is embedded within an $S U(5)$ global symmetry. The $Z^{\prime}$ that arises in this model couples purely to the left handed component of fermions, with couplings given by $L_{f}=T_{3}$, the third component of isospin, and $R_{f}=0$.

The coupling strength for the LH model is given by the following relation:

$$
\frac{g_{Z_{H}}}{g_{Z}}=\mathrm{c}_{W} \cot \theta_{H}
$$

where the parameter $\theta_{H}$ is defined as the mixing angle between the two $S U(2)$ groups of the model $\left(\tan \theta_{H}=g_{2} / g_{1}\right)$. In general, this is a free parameter; the value $\cot \theta_{H}=$ 1 will be used (yielding $\theta_{H}=45^{\circ}$ ) as this minimizes the branching fraction to $Z H$ and $W W$. Electroweak precision data suggests that the value of $\cot \theta_{H}$ is not large [63], which further supports this value.

\section{Simplest Little Higgs Model (SLH)}

The Simplest Little Higgs Model (SLH) [64-66] arises from a different gauge structure. In this model, the gauge group is expanded to $S U(3)_{W} \times U(1)_{\chi} \rightarrow S U(2)_{L} \times U(1)_{Y}$. 
This model includes five gauge bosons at large masses, including a neutral extra gauge boson. The couplings of the SM particles to the extra neutral sector are listed in Table A.2. The coupling strength is then given by the relation:

$$
\frac{g_{Z^{\prime}}}{g_{Z}}=\frac{1}{\sqrt{3-4 \mathrm{~s}_{W}}}
$$

Table A.2: Couplings of the left and right handed fermions to the $Z^{\prime}$ in the Simplest Little Higgs model.

\begin{tabular}{|c|c|c|}
\hline Fermion & $L_{f}$ & $R_{f}$ \\
\hline \hline$u, c, t$ & $\frac{1}{2}-\frac{1}{3} \mathrm{~s}_{W}^{2}$ & $\frac{2}{3} \mathrm{~s}_{W}^{2}$ \\
\hline$d, s, b$ & $\frac{1}{2}-\frac{1}{3} \mathrm{~s}_{W}^{2}$ & $-\frac{1}{3} \mathrm{~s}_{W}^{2}$ \\
\hline$e, \mu, \tau$ & $\frac{1}{2}-\mathrm{s}_{W}^{2}$ & $-\mathrm{s}_{W}^{2}$ \\
\hline$\nu_{e}, \nu_{\mu}, \nu_{\tau}$ & $\frac{1}{2}-\mathrm{s}_{W}^{2}$ & 0 \\
\hline
\end{tabular}

\section{Anomaly Free Simple Little Higgs Model (AFSLH)}

The Anomaly Free Simple Little Higgs Model (AFSLH) [63,67] arises similarly to the SLH model, but allows for couplings to the third generation quarks that are distinct from the first two generations in order to cancel out anomalies in loop diagrams. Lepton couplings remain identical throughout the generations. The coupling strength remains the same as in the SLH, but the model has $Z^{\prime}$ couplings as listed in Table A.3.

\section{A.3.4 3-3-1 Models (331)}

The name "3-3-1" $[68,69]$ is given to the set of models that arise from a group symmetry of $S U(3)_{c} \times S U(3) \times U(1)_{X}$. These models predict new physics close to the electroweak symmetry breaking scale, and draw a link between the number of colours and the number of fermion families. 
Table A.3: Couplings of the left and right handed fermions to the $Z^{\prime}$ in the Simple Little Higgs model with non-generation universal couplings.

\begin{tabular}{|c|c|c|}
\hline Fermion & $L_{f}$ & $R_{f}$ \\
\hline \hline$t$ & $\frac{1}{2}-\frac{1}{3} \mathrm{~s}_{W}^{2}$ & $\frac{2}{3} \mathrm{~s}_{W}^{2}$ \\
\hline$b$ & $\frac{1}{2}-\frac{1}{3} \mathrm{~s}_{W}^{2}$ & $-\frac{1}{3} \mathrm{~s}_{W}^{2}$ \\
\hline$u, c$ & $-\frac{1}{2}+\frac{2}{3} \mathrm{~s}_{W}^{2}$ & $\frac{2}{3} \mathrm{~s}_{W}^{2}$ \\
\hline$d, s$ & $-\frac{1}{2}+\frac{2}{3} \mathrm{~s}_{W}^{2}$ & $-\frac{1}{3} \mathrm{~s}_{W}^{2}$ \\
\hline$e, \mu, \tau$ & $\frac{1}{2}-\mathrm{s}_{W}^{2}$ & $-\mathrm{s}_{W}^{2}$ \\
\hline$\nu_{e}, \nu_{\mu}, \nu_{\tau}$ & $\frac{1}{2}-\mathrm{s}_{W}^{2}$ & 0 \\
\hline
\end{tabular}

Table A.4: Couplings of the SM Fermions in the 3-3-1 model

\begin{tabular}{|c|c|c|}
\hline Fermion & $L_{f}$ & $R_{f}$ \\
\hline \hline$c, t$ & $\frac{1}{2}-\frac{1}{3} \mathrm{~s}_{W}^{2}$ & $\frac{2}{3} \mathrm{~s}_{W}^{2}$ \\
\hline$s, b$ & $\frac{1}{2}-\frac{1}{3} \mathrm{~s}_{W}^{2}$ & $-\frac{1}{3} \mathrm{~s}_{W}^{2}$ \\
\hline$u$ & $-\frac{1}{2}+\frac{2}{3} \mathrm{~s}_{W}^{2}$ & $\frac{2}{3} \mathrm{~s}_{W}^{2}$ \\
\hline$d$ & $-\frac{1}{2}+\frac{2}{3} \mathrm{~s}_{W}^{2}$ & $-\frac{1}{3} \mathrm{~s}_{W}^{2}$ \\
\hline$e, \mu, \tau$ & $-\frac{1}{2}$ & $-\mathrm{s}_{W}^{2}$ \\
\hline$\nu_{e}, \nu_{\mu}, \nu_{\tau}$ & $\frac{1}{2}$ & $-\mathrm{s}_{W}^{2}$ \\
\hline
\end{tabular}

The couplings of the SM fermions are listed in Table A.4, with coupling strength of the model given by:

$$
\frac{g_{Z^{\prime}}}{g_{Z}}=\frac{1}{\sqrt{3-4 \mathrm{~s}_{W}^{2}}}
$$

From this, we can see that we require the relation $s_{W}^{2}<3 / 4$. Some 3-3-1 models include the stipulation that $s_{W}^{2}<1 / 4$ - surprisingly, the experimentally determined value of $\mathrm{s}_{W}^{2}$ is quite close to this upper bound. 


\section{Appendix B}

\section{Two Higgs Doublet Model Relations}

The potential given in Eq. 6.8 is similar to that of a two Higgs doublet model (2HDM) [125]. It can be shown that a four component Higgs field in $S O(4)\left(h=\left(h_{1}, h_{2}, h_{3}, h_{4}\right)\right)$ can be embedded in an $S U(2)$ doublet as:

$$
h=\frac{1}{\sqrt{2}}\left(\begin{array}{c}
h_{3}+i h_{4} \\
h_{1}-i h_{2}
\end{array}\right)
$$

From this, the link between the BLH Higgs potential and the well understood 2HDM potential is clear. This relationship means that many of the tools developed for 2HDMs can be used to exactly solve the Higgs potential in the BLH model.

For completeness, the minor contributions to Eq. 6.8 arising from ColemanWeinberg loops that were disregarded in the original BLH paper [3] should be included in the full potential. These terms and the relation to the general 2HDM are discussed in this appendix.

The fields $h_{1}, h_{2}, \eta^{a}$ and $\phi^{a}$ in this appendix are defined in Sec. 6.1.1. 


\section{B.1 Coleman-Weinberg Potential}

The Coleman-Weinberg (CW) potential arises from loops involving scalars, gauge bosons and fermions, as in Fig. 2.4. In general, the one loop quadratic and logarithmic contribution can be found from:

$$
V_{\text {oneloop }}=\frac{\Lambda^{2}}{32 \pi^{2}} \operatorname{Str}\left[M^{2}(\Sigma)\right]+\frac{1}{64 \pi^{2}} \operatorname{Str}\left[M^{4}(\Sigma)\left(\log \left(\frac{M^{2}(\Sigma)}{\Lambda^{2}}\right)-\frac{1}{2}\right)\right]
$$

where $\Lambda$ is the scale of new physics (typically taken as $\Lambda=4 \pi f$ ), and $M$ represents the

scalar mass matrix. The supertrace (Str) operator is given by $\operatorname{Str}[]=\sum_{s}(-1)^{2 s}(2 s+$ 1) $\operatorname{Tr}[$, where $s$ represents the spin of the particle in the loop, and the trace is taken over a matrix of the masses of the specific sector being examined.

These results are given in the original Bestest Little Higgs paper [3], and are included here for reference.

\section{Scalar Sector}

Scalar contributions to the CW potential arise from terms involving the operators $P_{i} \Sigma P_{j}$ (where $i, j=5,6$ ), as well as from the interaction terms arising from expanding $\Sigma$ in Eq. 6.7. However, the coefficients of the terms leading from Eq. 6.7 are expected to be very small and can be neglected.

The quadratically divergent contribution is given by:

$$
-\frac{3 f^{2} \Lambda^{2}}{16 \pi^{2}}\left(\lambda_{56}\left|\Sigma_{56}\right|^{2}+\lambda_{65}\left|\Sigma_{65}\right|^{2}\right)
$$

which is of the same size but opposite sign as the tree level contribution. The logarithmically divergent contribution is given by:

$$
\frac{\lambda_{56} \lambda_{65}}{16 \pi^{2}} \log \left(\frac{\Lambda^{2}}{m_{\sigma}^{2}}\right)\left[f^{2}\left(h_{1}^{T} h_{1}+h_{2}^{T} h_{2}\right)-\frac{2}{3}\left(h_{1}^{T} h_{1}\right)^{2}-\frac{2}{3}\left(h_{2}^{T} h_{2}\right)^{2}-2\left(h_{1}^{T} h_{1}\right)\left(h_{2}^{T} h_{2}\right)\right] .
$$




\section{Gauge Sector}

Loops involving gauge bosons arising from the $S U(2)_{A} \times S U(2)_{B}$ symmetries only contribute to the logarithmic $\mathrm{CW}$ potential, due to collective symmetry breaking, and give the following terms:

$$
\begin{aligned}
& -\frac{3 g_{A}^{2} g_{B}^{2} F^{2}}{64 \pi^{2} f^{2}} \log \left(\frac{\Lambda^{2}}{m_{W^{\prime}}^{2}}\right)\left[\frac{1}{16}\left(h_{1}^{T} h_{1}+h_{2}^{T} h_{2}\right)^{2}+\frac{1}{2} \phi_{a} \phi_{a}\left(h_{1}^{T} h_{1}+h_{2}^{T} h_{2}\right)+\frac{1}{3}\left(\phi_{a} \phi_{a}\right)^{2}\right] \\
& +\frac{9 g_{A}^{2} g_{B}^{2}\left(f^{2}+F^{2}\right)}{512 \pi^{2}} \log \left(\frac{\Lambda^{2}}{M_{W^{\prime}}^{2}}\right)\left[h_{1}^{T} h_{1}+h_{2}^{T} h_{2}+\frac{8}{3} \phi_{a} \phi_{a}\right]
\end{aligned}
$$

However, the hypercharge symmetry is not implemented collectively (there is only one $U(1)$ symmetry), and does contribute quadratically divergent terms. The hypercharge contribution to the $\mathrm{CW}$ potential is given by:

$$
\begin{array}{r}
-\frac{3 g_{Y}^{2} \Lambda^{2}}{32 \pi^{2} f^{2}}\left(\frac{1}{3}\left(\eta_{1}^{2}+\eta_{2}^{2}\right)^{2}+\frac{1}{3}\left(\eta_{1}^{2}+\eta_{2}^{2}\right) \eta_{3}^{2}+\frac{1}{2}\left(\eta_{1}^{2}+\eta_{2}^{2}\right)\left(h_{1}^{T} h_{1}+h_{2}^{T} h_{2}\right)\right. \\
\left.+8 \operatorname{Tr}\left[\frac{1}{192}\left(\left(h_{1}^{T} h_{1}\right)^{2}+\left(h_{2}^{T} h_{2}\right)^{2}+\left(h_{1}^{T} h_{2}\right)^{2}\right)+\frac{1}{4} \Pi_{h}^{2} T_{R}^{3} \Pi_{h}^{2} T_{R}^{3}\right]\right)
\end{array}
$$

The final term involving the $6 \times 6$ matrix, $\Pi_{h}$, does not contribute to electroweak symmetry breaking, and simply results in specific scalar interactions with a very small coupling. (Expanded out, this term gives $\operatorname{Tr}\left[\Pi_{h}^{2} T_{R}^{3} \Pi_{h}^{2} T_{R}^{3}\right]=1 / 8\left(h_{12} h_{21}-h_{11} h_{22}-\right.$ $\left.h_{14} h_{23}+h_{13} h_{24}\right)^{2}$.)

\section{Fermion Sector}

Due to collective symmetry breaking, the heavy top sector does not contribute either a quadratic or a logarithmically divergent term to the Higgs mass. It does, however, contribute a finite negative term to the $m_{1}$ parameter, which suggests the relation $\tan \beta>1$. This finite term is given by:

$$
-\frac{27 f^{2}}{16 \pi^{2}} \frac{\left|y_{1}\right|^{2}\left|y_{2}\right|^{2}\left|y_{3}\right|^{2}}{\left|y_{2}\right|^{2}-\left|y_{3}\right|^{2}} \log \left(\frac{\left|y_{1}\right|^{2}+\left|y_{2}\right|^{2}}{\left|y_{1}\right|^{2}+\left|y_{3}\right|^{2}}\right) h_{1}^{T} h_{1} .
$$




\section{B.2 Relation to $2 \mathrm{HDM}$}

The general BLH potential, including all possible Higgs interactions, can be written as:

$$
\begin{aligned}
V= & \frac{1}{2} m_{1}^{2} h_{1}^{T} h_{1}+\frac{1}{2} m_{2}^{2} h_{2}^{T} h_{2}-B_{\mu} h_{1}^{T} h_{2}+\frac{1}{2} \lambda_{0}\left(h_{1}^{T} h_{2}\right)^{2}+\frac{1}{2} \lambda^{\prime}\left(h_{1}^{T} h_{1}\right)\left(h_{2}^{T} h_{2}\right)+\frac{1}{2} \lambda_{1}\left(h_{1}^{T} h_{1}\right)^{2} \\
& +\frac{1}{2} \lambda_{2}\left(h_{2}^{T} h_{2}\right)^{2}+\frac{1}{2} \lambda_{3}\left(h_{1}^{T} h_{2}\right)\left(h_{1}^{T} h_{1}\right)+\frac{1}{2} \lambda_{4}\left(h_{1}^{T} h_{2}\right)\left(h_{2}^{T} h_{2}\right)
\end{aligned}
$$

This potential contains all possible combinations of the mass and quartic couplings for the two Higgs fields, $h_{1}$ and $h_{2}$, that arise at tree and/or loop level.

The general 2 HDM potential can be written as [125]:

$$
\begin{aligned}
V= & m_{11}^{2} \phi_{1}^{\dagger} \phi_{1}+m_{22}^{2} \phi_{2}^{\dagger} \phi_{2}-m_{12}^{2}\left(\phi_{1}^{\dagger} \phi_{2}+\phi_{2}^{\dagger} \phi_{1}\right)+\frac{1}{2} \lambda_{1}\left(\phi_{1}^{\dagger} \phi_{1}\right)^{2}+\frac{1}{2} \lambda_{2}\left(\phi_{2}^{\dagger} \phi_{2}\right)^{2} \\
& +\lambda_{3}\left(\phi_{1}^{\dagger} \phi_{1}\right)\left(\phi_{2}^{\dagger} \phi_{2}\right)+\lambda_{4}\left(\phi_{2}^{\dagger} \phi_{1}\right)\left(\phi_{1}^{\dagger} \phi_{2}\right)+\frac{1}{2} \lambda_{5}\left(\left(\phi_{1}^{\dagger} \phi_{2}\right)\left(\phi_{1}^{\dagger} \phi_{2}\right)+\left(\phi_{2}^{\dagger} \phi_{1}\right)\left(\phi_{2}^{\dagger} \phi_{1}\right)\right) \\
& +\left(\lambda_{6} \phi_{1}^{\dagger} \phi_{1}+\lambda_{7} \phi_{2}^{\dagger} \phi_{2}\right)\left(\phi_{1}^{\dagger} \phi_{2}+\phi_{2}^{\dagger} \phi_{1}\right)
\end{aligned}
$$

By expanding out the two potentials, the relationship between the parameter sets becomes clear:

$$
\begin{aligned}
\beta_{(2 h d m)} & =-\beta_{(b l h)}+\pi / 2 \\
\alpha_{(2 h d m)} & =\alpha_{(b l h)}-\pi / 2 \\
h_{0(2 h d m)} & =-h_{0(b l h)} \\
m_{12(2 h d m)}^{2} & =B_{\mu(b l h)} \\
m_{11(2 h d m)} & =m_{1(b l h)} \\
m_{22(2 h d m)} & =m_{2(b l h)}
\end{aligned}
$$




$$
\begin{aligned}
\lambda_{1(2 h d m)} / 4 & =\lambda_{1(b l h)} \\
\lambda_{2(2 h d m)} / 4 & =\lambda_{2(b l h)} \\
\lambda_{3(2 h d m)} / 2 & =\lambda_{(b l h)}^{\prime} \\
\lambda_{4(2 h d m)} / 2+\lambda_{5(2 h d m)} / 2 & =\lambda_{0(b l h)} \\
\lambda_{4(2 h d m)} & =\lambda_{5(2 h d m)} \\
\lambda_{6(2 h d m)} & =\lambda_{3(b l h)} \\
\lambda_{7(2 h d m)} & =\lambda_{4(b l h)}
\end{aligned}
$$

By considering the tree level contributions to the scalar potential and the CW contributions, the BLH-2HDM parameters can be related to the fundamental parameters of the model through the following relations:

$$
\begin{aligned}
\lambda_{\sigma}= & \frac{\lambda_{65}-\lambda_{56}}{\sqrt{2}\left(\lambda_{56}+\lambda_{65}\right)} \\
m_{1}^{2}= & \frac{9\left(f^{2}+F^{2}\right) g_{A}^{2} g_{B}^{2}}{256 \pi^{2}} \log \left[\frac{\Lambda^{2}}{M_{W^{\prime}}^{2}}\right]+\frac{f^{2} \lambda_{56} \lambda_{65}}{8 \pi^{2}} \log \left[\frac{\Lambda^{2}}{M_{\sigma}^{2}}\right]+\frac{3 g_{Y}^{2} \Lambda^{2}}{64 \pi^{2}} \\
& -\frac{27 f^{2} y_{1}^{2} y_{2}^{2} y_{3}^{2}}{8 \pi^{2}\left(y_{2}^{2}-y_{3}^{2}\right)} \log \left[\frac{y_{1}^{2}+y_{2}^{2}}{y_{1}^{2}+y_{3}^{2}}\right]+\frac{1}{2} m_{4}^{2}+\frac{1}{2} m_{5}^{2} \\
m_{2}^{2}= & \frac{9\left(f^{2}+F^{2}\right) g_{A}^{2} g_{B}^{2}}{256 \pi^{2}} \log \left[\frac{\Lambda^{2}}{M_{W^{\prime}}^{2}}\right]+\frac{f^{2} \lambda_{56} \lambda_{65}}{8 \pi^{2}} \log \left[\frac{\Lambda^{2}}{M_{\sigma}^{2}}\right]+\frac{3 g_{Y}^{2} \Lambda^{2}}{64 \pi^{2}} \\
& +\frac{1}{2} m_{4}^{2}+\frac{1}{2} m_{6}^{2} \\
B_{\mu}= & m_{56}^{2}\left(1+\sqrt{2} \lambda_{\sigma}\right)+m_{65}^{2}\left(1-\sqrt{2} \lambda_{\sigma}\right) \\
\lambda_{0}= & \frac{2 \lambda_{56} \lambda_{65}}{\lambda_{56}+\lambda_{65}}-\frac{m_{4}^{2}}{6 f^{2}}-\frac{m_{5}^{2}\left(1-6 \lambda_{\sigma}^{2}\right)}{12 f^{2}}-\frac{m_{6}^{2}\left(1-6 \lambda_{\sigma}^{2}\right)}{12 f^{2}}+\frac{g_{Y}^{2} \Lambda^{2}}{32 \pi^{2} f^{2}}
\end{aligned}
$$




$$
\begin{aligned}
\lambda^{\prime}= & -\frac{3 g_{Y}^{2} \Lambda^{2}}{64 \pi^{2} f^{2}}-\frac{3 F^{2} g_{A}^{2} g_{B}^{2}}{256 \pi^{2} f^{2}} \log \left[\frac{\Lambda^{2}}{M_{W^{\prime}}^{2}}\right]-\frac{\lambda_{56} \lambda_{65}}{4 \pi^{2}} \log \left[\frac{\Lambda^{2}}{M_{\sigma}^{2}}\right] \\
\lambda_{1}= & -\frac{g_{Y}^{2} \Lambda^{2}}{128 \pi^{2} f^{2}}-\frac{3 F^{2} g_{A}^{2} g_{B}^{2}}{512 \pi^{2} f^{2}} \log \left[\frac{\Lambda^{2}}{M_{W^{\prime}}^{2}}\right]-\frac{\lambda_{56} \lambda_{65}}{12 \pi^{2}} \log \left[\frac{\Lambda^{2}}{M_{\sigma}^{2}}\right] \\
& -\frac{m_{4}^{2}}{12 f^{2}}-\frac{m_{5}^{2}}{12 f^{2}} \\
\lambda_{2}= & -\frac{g_{Y}^{2} \Lambda^{2}}{128 \pi^{2} f^{2}}-\frac{3 F^{2} g_{A}^{2} g_{B}^{2}}{512 \pi^{2} f^{2}} \log \left[\frac{\Lambda^{2}}{M_{W^{\prime}}^{2}}\right]-\frac{\lambda_{56} \lambda_{65}}{12 \pi^{2}} \log \left[\frac{\Lambda^{2}}{M_{\sigma}^{2}}\right] \\
& -\frac{m_{4}^{2}}{12 f^{2}}-\frac{m_{6}^{2}}{12 f^{2}} \\
\lambda_{3}= & \frac{1}{3 f^{2}}\left(m_{65}^{2}\left(1-3 \sqrt{2} \lambda_{\sigma}\right)+m_{56}^{2}\left(1+3 \sqrt{2} \lambda_{\sigma}\right)\right) \\
\lambda_{4}= & \frac{1}{3 f^{2}}\left(m_{65}^{2}\left(1-3 \sqrt{2} \lambda_{\sigma}\right)+m_{56}^{2}\left(1+3 \sqrt{2} \lambda_{\sigma}\right)\right)
\end{aligned}
$$

Relating the $\mathrm{BLH}$ potential to a $2 \mathrm{HDM}$ potential provides insight into the nature of the parameters in the BLH model based on studies on the 2HDM. For example, the following constraints exist for any generic $2 \mathrm{HDM}$, and can be applied to the $\mathrm{BLH}$ model $[143,144]$ :

$$
\begin{aligned}
\lambda_{1} & >0 \\
\lambda_{2} & >0 \\
\lambda^{\prime} & >-2 \sqrt{\lambda_{1} \lambda_{2}} \\
\lambda^{\prime} / 2+\lambda_{0} / 4-\left|\lambda_{0} / 4\right| & >-\sqrt{\lambda_{1} \lambda_{2}} \\
\left|\lambda_{i}\right| & <4 \pi
\end{aligned}
$$




$$
\begin{aligned}
m_{h} & <600 \mathrm{GeV} \\
m_{A} & <872 \mathrm{GeV} \\
m_{H} & <867 \mathrm{GeV} \\
m_{H^{ \pm}} & <779 \mathrm{GeV}
\end{aligned}
$$




\section{Appendix C}

\section{Mass Eigenstates in the Bestest Little Higgs Model}

This appendix lists the relationships between the fundamental fields in the Bestest Little Higgs model and the mass eigenstates found from diagonalizing the mass matrices to order $\left(v_{E W} / f\right)^{2}$ in the region where $y_{2} \neq y_{3}$.

\section{C.1 Scalar Sector}

The first components of the Higgs fields, $h_{11}$ and $h_{21}$, mix differently than the other components due to extra terms involving the vev.

$$
\begin{aligned}
& h_{11}=\cos \alpha h_{0}-\sin \alpha H_{0} \\
& h_{21}=\sin \alpha h_{0}+\cos \alpha H_{0}
\end{aligned}
$$

The mixing angle, $\alpha$ is given by the relation [3]:

$$
\begin{aligned}
\tan \alpha= & \frac{1}{B_{\mu}-\lambda_{0} v_{E W}^{2} \sin 2 \beta}\left(B_{\mu} \cot 2 \beta\right. \\
& \left.+\sqrt{\frac{B_{\mu}^{2}}{\sin ^{2} 2 \beta}-2 \lambda_{0} B_{\mu} v_{E W}^{2} \sin 2 \beta+\lambda_{0}^{2} v_{E W}^{4} \sin ^{2} 2 \beta}\right)
\end{aligned}
$$


The other components of the Higgs fields are given by:

$$
\begin{aligned}
& h_{12}=\sin \beta G_{0}-\cos \beta A_{0} \\
& h_{22}=\cos \beta G_{0}+\sin \beta A_{0} \\
& h_{13}=\frac{1}{\sqrt{2}}\left(\sin \beta\left(G^{-}+G^{+}\right)-\cos \beta\left(H^{-}+H^{+}\right)\right) \\
& h_{14}=\frac{i}{\sqrt{2}}\left(\sin \beta\left(G^{-}-G^{+}\right)-\cos \beta\left(H^{-}-H^{+}\right)\right) \\
& h_{23}=\frac{1}{\sqrt{2}}\left(\cos \beta\left(G^{-}+G^{+}\right)+\sin \beta\left(H^{-}+H^{+}\right)\right) \\
& h_{14}=\frac{i}{\sqrt{2}}\left(\cos \beta\left(G^{-}-G^{+}\right)+\sin \beta\left(H^{-}-H^{+}\right)\right)
\end{aligned}
$$

where $\tan \beta=\left\langle h_{1}\right\rangle /\left\langle h_{2}\right\rangle$, as before.

The two triplets under the $S U(2)_{L}$ symmetry mix to form a physical $\left(\phi^{\prime}\right)$ and eaten $\left(\chi^{\prime}\right)$ triplet:

$$
\begin{aligned}
& \phi_{1}=\frac{1}{\sqrt{2} \sqrt{f^{2}+F^{2}}}\left(F\left(\left(\phi^{\prime}\right)^{-}+\left(\phi^{\prime}\right)^{+}\right)+f\left(\left(\chi^{\prime}\right)^{-}+\left(\chi^{\prime}\right)^{+}\right)\right) \\
& \phi_{2}=\frac{i}{\sqrt{2} \sqrt{f^{2}+F^{2}}}\left(-F\left(\left(\phi^{\prime}\right)^{-}-\left(\phi^{\prime}\right)^{+}\right)-f\left(\left(\chi^{\prime}\right)^{-}-\left(\chi^{\prime}\right)^{+}\right)\right) \\
& \phi_{3}=\frac{1}{\sqrt{f^{2}+F^{2}}}\left(F\left(\phi^{\prime}\right)^{0}+f\left(\chi^{\prime}\right)^{0}\right) \\
& \chi_{1}=\frac{1}{\sqrt{2} \sqrt{f^{2}+F^{2}}}\left(-f\left(\left(\phi^{\prime}\right)^{-}+\left(\phi^{\prime}\right)^{+}\right)+F\left(\left(\chi^{\prime}\right)^{-}+\left(\chi^{\prime}\right)^{+}\right)\right) \\
& \chi_{2}=\frac{i}{\sqrt{2} \sqrt{f^{2}+F^{2}}}\left(f\left(\left(\phi^{\prime}\right)^{-}-\left(\phi^{\prime}\right)^{+}\right)-F\left(\left(\chi^{\prime}\right)^{-}-\left(\chi^{\prime}\right)^{+}\right)\right) \\
& \chi_{3}=\frac{1}{\sqrt{f^{2}+F^{2}}}\left(-f\left(\phi^{\prime}\right)^{0}+F\left(\chi^{\prime}\right)^{0}\right)
\end{aligned}
$$

The only mixing for the $S U(2)_{R}$ triplet results in the charge eigenstates, as is typical of $S U(2)$ triplets:

$$
\begin{aligned}
& \eta_{1}=\frac{1}{\sqrt{2}}\left(\eta^{+}+\eta^{-}\right) \\
& \eta_{2}=\frac{i}{\sqrt{2}}\left(\eta^{+}-\eta^{-}\right) \\
& \eta_{3}=\eta^{0}
\end{aligned}
$$

No other mixing occurs for the $\eta$ fields. 


\section{C.2 Gauge Sector}

The Gauge sector consists of two $S U(2)$ triplets, $A_{1}$ and $A_{2}$, and a singlet, $B_{3}$, which gauges the $U(1)$ symmetry. These states, in terms of the mass eigenstates are given by:

$$
\begin{aligned}
A_{1}^{1}= & \frac{1}{\sqrt{2}}\left(\left(\cos \theta_{g} \cos \theta^{\prime \prime}-\frac{\cos \theta_{w} \sin \theta_{g} x_{s} v_{E W}^{2}}{f^{2}+F^{2}}\right)\left(W^{-}+W^{+}\right)\right. \\
& \left.+\left(\sin \theta_{g} \cos \theta^{\prime \prime}+\frac{\cos \theta_{g} \cos \theta_{w} x_{s} v_{E W}^{2}}{f^{2}+F^{2}}\right)\left(W^{\prime-}+W^{\prime+}\right)\right) \\
A_{1}^{2}= & \frac{i}{\sqrt{2}}\left(\left(\cos \theta_{g} \cos \theta^{\prime \prime}-\frac{\cos \theta_{w} \sin \theta_{g} x_{s} v_{E W}^{2}}{f^{2}+F^{2}}\right)\left(-W^{-}+W^{+}\right)\right. \\
& \left.+\left(\sin \theta_{g} \cos \theta^{\prime \prime}+\frac{\cos \theta_{g} \cos \theta_{w} x_{s} v_{E W}^{2}}{f^{2}+F^{2}}\right)\left(-W^{\prime-}+W^{\prime+}\right)\right) \\
A_{2}^{1}= & \frac{1}{\sqrt{2}}\left(\left(\sin \theta_{g} \cos \theta^{\prime \prime}+\frac{\cos \theta_{g} \cos \theta_{w} x_{s} v_{E W}^{2}}{f^{2}+F^{2}}\right)\left(W^{-}+W^{+}\right)\right. \\
& \left.-\left(\cos \theta_{g} \cos \theta^{\prime \prime}-\frac{\cos \theta_{w} \sin \theta_{g} x_{s} v_{E W}^{2}}{f^{2}+F^{2}}\right)\left(W^{\prime-}+W^{\prime+}\right)\right) \\
A_{2}^{2}= & \frac{i}{\sqrt{2}}\left(\left(\sin \theta_{g} \cos \theta^{\prime \prime}+\frac{\cos \theta_{g} \cos \theta_{w} x_{s} v_{E W}^{2}}{f^{2}+F^{2}}\right)\left(-W^{-}+W^{+}\right)\right. \\
A_{2}^{3}= & \sin \theta_{g} \sin \theta_{w} A_{\gamma}+\left(\sin \theta_{g} \cos \theta_{w} \cos \theta^{\prime}+\frac{\cos \theta_{g} x_{s} v_{E W}^{2}}{f^{2}+F^{2}}\right) Z^{0} \\
& \left.-\left(\cos \theta_{g} \cos \theta^{\prime \prime}-\frac{\cos \theta_{w} \sin \theta_{g} x_{s} v_{E W}^{2}}{f^{2}+F^{2}}\right)\left(-W^{\prime-}+W^{\prime+}\right)\right) \\
A_{1}^{3}= & \cos \theta_{g} \sin \theta_{w} A_{\gamma}+\left(\cos \theta_{g} \cos \theta_{w} \cos \theta^{\prime}-\frac{\sin \theta_{g} x_{s} v_{E W}^{2}}{f^{2}+F^{2}}\right) Z^{0} \\
+ & \left(\sin \theta_{g} \cos \theta^{\prime}+\frac{\cos \theta_{g} \cos \theta_{w} x_{s} v_{E W}^{2}}{f^{2}+F^{2}}\right) Z^{\prime} \\
& \\
&
\end{aligned}
$$




$$
\begin{gathered}
-\left(\cos \theta_{g} \cos \theta^{\prime}-\frac{\sin \theta_{g} \cos \theta_{w} x_{s} v_{E W}^{2}}{f^{2}+F^{2}}\right) Z^{\prime} \\
B_{3}=\cos \theta_{w} A_{\gamma}-\sin \theta_{w} \cos \theta^{\prime} Z^{0}-\frac{\sin \theta_{w} x_{s} v_{E W}^{2}}{f^{2}+F^{2}} Z^{\prime}
\end{gathered}
$$

In this simplified notation, the following parameters are used (in addition to the relations $\tan \theta_{g}=g_{A} / g_{B}$ and $\left.\tan \theta_{W}=g_{Y} / g\right)$ :

$$
\begin{aligned}
x_{s} & =\frac{1}{2 \cos \theta_{w}} \sin \theta_{g} \cos \theta_{g}\left(\sin ^{2} \theta_{g}-\cos ^{2} \theta_{g}\right) \\
\cos \theta^{\prime} & =1-\frac{1}{2} \frac{v_{E W}^{2}}{f^{2}+F^{2}} x_{s} \\
\cos \theta^{\prime \prime} & =1-\frac{1}{2} \frac{v_{E W}^{2}}{f^{2}+F^{2}} x_{s} \cos \theta_{w}
\end{aligned}
$$




\section{C.3 Fermion Sector}

At tree level, the interaction eigenstates and notation used in this thesis are related to the fundamental fields by:

$$
\begin{aligned}
Q_{a 1} & =\left(y_{1} Q_{u}+y_{2} q_{31}\right) / \sqrt{y_{1}^{2}+y_{2}^{2}} \\
Q_{a 1}^{\prime} & =\left(y_{2} Q_{u}-y_{1} q_{31}\right) / \sqrt{y_{1}^{2}+y_{2}^{2}} \\
Q_{a 2} & =\left(y_{1} Q_{d}+y_{2} q_{32}\right) / \sqrt{y_{1}^{2}+y_{2}^{2}} \\
Q_{a 2}^{\prime} & =\left(y_{2} Q_{d}-y_{1} q_{32}\right) / \sqrt{y_{1}^{2}+y_{2}^{2}} \\
Q_{b 1} & =Q_{b}^{5 / 3} \\
Q_{b 2} & =Q_{b}^{2 / 3} \\
U_{a 1} & =-U_{d} \\
U_{a 2} & =U_{a} \\
U_{b 1} & =U_{b}^{2 / 3} \\
U_{b 2} & =-U_{b}^{5 / 3} \\
U_{5} & =\left(y_{1} U_{q 5}+y_{3} u_{3}\right) / \sqrt{y_{1}^{2}+y_{3}^{2}} \\
U_{5}^{\prime} & =\left(y_{3} U_{q 5}-y_{1} u_{3}\right) / \sqrt{y_{1}^{2}+y_{3}^{2}}
\end{aligned}
$$

Extensive mixing occurs between the charge $+2 / 3$ fermion states. Additionally, a tree level degeneracy occurs when the Yukawa couplings $y_{2}$ and $y_{3}$ have approximately the same value. As such, the following analytic relations only hold when $y_{2}$ and $y_{3}$ are distinct.

$$
\begin{aligned}
q_{31}= & t-\frac{v_{E W}}{f}\left(\frac{2 \cos \beta y_{2}}{\sqrt{y_{1}^{2}+y_{2}^{2}}}\right) T_{6}-\frac{v_{E W}}{f}\left(\frac{\sin \beta y_{2}\left(2 y_{1}^{2}-y_{3}^{2}\right)}{\sqrt{y_{1}^{2}+y_{2}^{2}}\left(y_{1}^{2}+y_{3}^{2}\right)}\right) T_{5} \\
& -\frac{v_{E W}^{2}}{f^{2}}\left(\frac{(\cos 2 \beta-3) y_{2}}{\sqrt{y_{1}^{2}+y_{2}^{2}}}\right) T_{b}^{2 / 3} \\
& -\frac{v_{E W}^{2}}{f^{2}}\left(\frac{\left.y_{2}^{2}\left(8 y_{1}^{4}+4(1+3 \cos 2 \beta) y_{1}^{2} y_{3}^{2}+(5+3 \cos 2 \beta) y_{3}^{4}\right)\right)}{4\left(y_{1}^{2}+y_{2}^{2}\right)\left(y_{1}^{2}+y_{3}^{2}\right)^{2}}\right) t
\end{aligned}
$$




$$
\begin{aligned}
& -\frac{v_{E W}^{2}}{f^{2}}\left(\frac{y_{1} y_{2}}{2\left(y_{1}^{2}+y_{2}^{2}\right)^{2}\left(y_{2}^{2}-y_{3}^{2}\right)}\right)\left(2 y_{1}^{2}\left(2 y_{2}^{2}+(1-3 \cos 2 \beta) y_{3}^{2}\right)\right. \\
& \left.+y_{2}^{2}\left(4 y_{2}^{2}+(3 \cos 2 \beta-7) y_{3}^{2}\right)\right) T
\end{aligned}
$$

$$
\begin{aligned}
& Q_{u}=T-\frac{v_{E W}}{f}\left(\frac{2 \cos \beta y_{1}}{\sqrt{y 1^{2}+y_{2}^{2}}}\right) T_{6}-\frac{v_{E W}}{f}\left(\frac{\sin \beta y_{1}\left(2 y_{2}^{2}+y_{3}^{2}\right)}{\sqrt{y_{1}^{2}+y_{2}^{2}}\left(y_{2}^{2}-y_{3}^{2}\right)}\right) T_{5} \\
& -\frac{v_{E W}^{2}}{f^{2}}\left(\frac{(\cos 2 \beta-3) y_{1}}{\sqrt{y_{1}^{2}+y_{2}^{2}}}\right) T_{b}^{2 / 3} \\
& -\frac{v_{E W}^{2}}{f^{2}}\left(\frac{y_{1}^{2}\left(8 y_{2}^{4}-4(1+3 \cos 2 \beta) y_{2}^{2} y_{3}^{2}+(5+3 \cos 2 \beta) y_{3}^{4}\right)}{4\left(y_{1}^{2}+y_{2}^{2}\right)\left(y_{2}^{2}-y_{3}^{2}\right)^{2}}\right) T \\
& -\frac{v_{E W}^{2}}{f^{2}}\left(\frac{y_{1} y_{2}}{2\left(y_{1}^{2}+y_{2}^{2}\right)^{2}\left(y_{1}^{2}+y 3^{2}\right)}\right)\left(4 y_{1}^{4}+2(3 \cos 2 \beta-1) y_{2}^{2} y_{3}^{2}\right. \\
& \left.+y_{1}^{2}\left(4 y_{2}^{2}+(7-3 \cos 2 \beta) y_{3}^{2}\right)\right) t \\
& Q_{b}^{2 / 3}=T_{b}^{2 / 3}+\frac{v_{E W}}{f}(\sin \beta) T_{5} \\
& -\frac{v_{E W}^{2}}{f^{2}}\left(\frac{\sin ^{2} \beta}{2}\right) T_{b}^{2 / 3}-\frac{v_{E W}^{2}}{f^{2}}\left(\frac{y_{2}\left(2 y_{1}^{2}+\left(2+3 \sin ^{2} \beta\right) y_{3}^{2}\right)}{\sqrt{y_{1}^{2}+y_{2}^{2}}\left(y_{1}^{2}+y_{3}^{2}\right)}\right) t \\
& +\frac{v_{E W}^{2}}{f^{2}}\left(\frac{y_{1}\left(-2 y_{2}^{2}+\left(2+3 \sin ^{2} \beta\right) y_{3}^{2}\right)}{\sqrt{y_{1}^{2}+y_{2}^{2}}\left(y_{2}^{2}-y_{3}^{2}\right)}\right) T \\
& Q_{5}=T_{5}-\frac{v_{E W}}{f}(\sin \beta) T_{b}^{2 / 3}+\frac{v_{E W}}{f}\left(\frac{\sin \beta y_{2}\left(2 y_{1}^{2}-y_{3}^{2}\right)}{\sqrt{y_{1}^{2}+y_{2}^{2}}\left(y_{1}^{2}+y_{3}^{2}\right)}\right) t \\
& +\frac{v_{E W}}{f}\left(\frac{\sin \beta y_{1}\left(2 y_{2}^{2}+y_{3}^{2}\right)}{\sqrt{y_{1}^{2}+y_{2}^{2}}\left(y_{2}^{2}-y_{3}^{2}\right)}\right) T+\frac{v_{E W}^{2}}{f^{2}}(3 \cos \beta \sin \beta) T_{6}
\end{aligned}
$$




$$
\begin{aligned}
& -\frac{v_{E W}^{2}}{f^{2}}\left(\frac { \operatorname { s i n } ^ { 2 } \beta } { 2 ( y _ { 2 } ^ { 2 } - y _ { 3 } ^ { 2 } ) ^ { 2 } ( y _ { 1 } ^ { 2 } + y _ { 3 } ^ { 2 } ) ^ { 2 } } \left(2 y_{3}^{4}\left(y_{2}^{2}-y_{3}^{2}\right)^{2}+y_{1}^{4}\left(5 y_{2}^{4}+2 y_{2}^{2} y_{3}^{2}+2 y_{3}^{4}\right)\right.\right. \\
& \left.\left.+y_{1}^{2}\left(-2 y_{2}^{4} y_{3}^{2}+7 y_{2}^{2} y_{3}^{4}+4 y_{3}^{6}\right)\right)\right) T_{5} \\
& Q_{6}=T_{6}+\frac{v_{E W}}{f}\left(\frac{2 \cos \beta y_{1}}{\sqrt{y_{1}^{2}+y_{2}^{2}}}\right) T+\frac{v_{E W}}{f}\left(\frac{2 \cos \beta y_{2}}{\sqrt{y_{1}^{2}+y_{2}^{2}}}\right) t-\frac{v_{E W}^{2}}{f^{2}}\left(2 \cos ^{2} \beta\right) T_{6} \\
& -\frac{v_{E W}^{2}}{f^{2}}\left(\frac{\cos \beta \sin \beta\left(y_{3}^{2}\left(y_{2}^{2}-y_{3}^{2}\right)+y_{1}^{2}\left(7 y_{2}^{2}-y_{3}^{2}\right)\right)}{\left(y_{1}^{2}+y_{3}^{2}\right)\left(y_{2}^{2}-y_{3}^{2}\right)}\right) T_{5} \\
& Q_{d}=B \\
& Q_{b}^{5 / 3}=T_{b}^{5 / 3} \\
& u_{3}=t-\frac{v_{E W}}{f}\left(\frac{2 \sin \beta y_{3}}{\sqrt{y_{1}^{2}+y_{3}^{2}}}\right) T_{b}^{2 / 3}-\frac{v_{E W}}{f}\left(\frac{\sin \beta y_{3}\left(2 y_{1}^{2}-y_{2}^{2}\right)}{\sqrt{y_{1}^{2}+y_{3}^{2}}\left(y_{1}^{2}+y_{2}^{2}\right)}\right) T \\
& -\frac{v_{E W}^{2}}{f^{2}}\left(\frac{\sin ^{2} \beta\left(8 y_{1}^{4}+4 y_{1}^{2} y_{2}^{2}+5 y_{2}^{4}\right) y_{3}^{2}}{2\left(y_{1}^{2}+y_{2}^{2}\right)^{2}\left(y_{1}^{2}+y_{3}^{2}\right)}\right) t+\frac{v_{E W}^{2}}{f^{2}}\left(\frac{6 \cos \beta \sin \beta y_{3}}{\sqrt{y_{1}^{2}+y_{3}^{2}}}\right) T_{6} \\
& -\frac{v_{E W}^{2}}{f^{2}}\left(\frac{\sin ^{2} \beta y_{1} y_{3}\left(-7 y_{2}^{2} y_{3}^{2}+4 y_{3}^{4}+2 y_{1}^{2}\left(y_{2}^{2}+2 y_{3}^{2}\right)\right)}{\left(y_{1}^{2}+y_{3}^{2}\right)^{2}\left(-y_{2}^{2}+y_{3}^{2}\right)}\right) T_{5} \\
& U_{u}=T-\frac{v_{E W}}{f}(\cos \beta) T_{6}+\frac{v_{E W}}{f}\left(\frac{\sin \beta y_{3}\left(2 y_{1}^{2}-y_{2}^{2}\right)}{2\left(y_{1}^{2}+y_{2}^{2}\right)^{2}\left(y_{1}^{2}+y_{3}^{2}\right)}\right) t \\
& +\frac{v_{E W}}{f}\left(\frac{\sin \beta y_{1}\left(y_{2}^{2}+2 y_{3}^{2}\right)}{\sqrt{y_{1}^{2}+y_{3}^{2}}\left(-y_{2}^{2}+y_{3}^{2}\right)}\right) T_{5}+\frac{v_{E W}^{2}}{f^{2}}\left(\frac{\left(1+4 \sin ^{2} \beta\right)}{2}\right) T_{b}^{2 / 3} \\
& +\frac{v_{E W}^{2}}{f^{2}}\left(\frac { 1 } { 4 ( y _ { 1 } ^ { 2 } + y _ { 2 } ^ { 2 } ) ^ { 2 } ( y _ { 2 } ^ { 2 } - y _ { 3 } ^ { 2 } ) ^ { 2 } } \left(-2 y_{2}^{4}\left(y_{2}^{2}-y_{3}^{2}\right)^{2}+y_{1}^{4}\left(-2 y_{2}^{4}\right.\right.\right. \\
& \left.+2(-1+3 \cos 2 \beta) y_{2}^{2} y_{3}^{2}+(-5+3 \cos 2 \beta) y_{3}^{4}\right)-y_{1}^{2} y_{2}^{2}\left(4 y_{2}^{4}-\right.
\end{aligned}
$$




$$
\begin{aligned}
&\left.\left.\left.(-7+15 \cos 2 \beta) y_{2}^{2} y_{3}^{2}+2(-1+3 \cos 2 \beta) y_{3}^{4}\right)\right)\right) T \\
& U_{b}^{2 / 3}= T_{b}^{2 / 3}+\frac{v_{E W}}{f}\left(\frac{2 \sin \beta y_{1}}{\sqrt{y_{1}^{2}+y_{3}^{2}}}\right) T_{5}+\frac{v_{E W}}{f}\left(\frac{2 \sin \beta y_{3}}{\sqrt{y_{1}^{2}+y_{3}^{2}}}\right) t-\frac{v_{E W}^{2}}{f^{2}}\left(2 \sin ^{2} \beta\right) T_{b}^{2 / 3} \\
&-\frac{v_{E W}^{2}}{f^{2}}\left(\frac{\left(y_{2}^{4}-y_{2}^{2} y_{3}^{2}+y 1^{2}\left(y_{2}^{2}+(-7+6 \cos 2 \beta) y_{3}^{2}\right)\right)}{2\left(y_{1}^{2}+y_{2}^{2}\right)\left(y_{2}^{2}-y_{3}^{2}\right)}\right) T \\
& U_{q 5}= T_{5}+\frac{v_{E W}}{f}\left(\frac{2 \sin \beta y_{1}}{\sqrt{y_{1}^{2}+y_{3}^{2}}}\right) T_{b}^{2 / 3}+\frac{v_{E W}}{f}\left(\frac{2 \sin \beta y_{3}}{\sqrt{y_{1}^{2}+y_{3}^{2}}}\right) t \\
&+\frac{v_{E W}^{2}}{f^{2}}\left(\frac{6 \cos \beta \sin \beta y_{1}}{\sqrt{y_{1}^{2}+y_{3}^{2}}}\right) T_{6}-\frac{v_{E W}^{2}}{f^{2}}\left(\frac{\sin ^{2} \beta y_{1}^{2}\left(5 y_{2}^{4}-4 y_{2}^{2} y_{3}^{2}+8 y_{3}^{4}\right)}{2\left(y_{2}^{2}-y_{3}^{2}\right)\left(y_{1}^{2}+y_{3}^{2}\right)}\right) T_{5} \\
&-\frac{v_{E W}^{2}}{f^{2}}\left(\frac{\sin ^{2} \beta y_{1} y_{3}\left(4 y_{1}^{4}-2 y_{2} y_{3}^{2}+y_{1}^{2}\left(7 y_{2}^{2}+4 y_{3}^{2}\right)\right)}{\left(y_{1}^{2}+y_{2}^{2}\right)\left(y_{1}^{2}+y_{3}^{2}\right)^{2}}\right) t \\
& U_{6}= T_{6}+\frac{v_{E W}}{f}(\cos \beta) T \\
& U_{b}^{5 / 3}=\left.T_{b}^{5 / 3} \quad \text { (C. } .52\right) \\
&-\frac{v_{E W}^{2}}{f^{2}}\left(\frac{\cos { }^{2} \beta}{2}\right) T_{6}-\frac{v_{E W}^{2}}{f^{2}}\left(\frac{\cos \beta \sin \beta\left(4 y_{1}^{2}+7 y_{2}^{2}\right) y_{3}}{\left.\left(y_{1}^{2}+y_{2}^{2}\right) \sqrt{y_{1}^{2}+y_{3}^{2}}\right) t}\right. \\
&+\frac{v_{E W}^{2}}{f^{2}}\left(\frac{\cos \beta \sin \beta y_{1}\left(7 y_{2}^{2}-4 y_{3}^{2}\right)}{\left.\sqrt{y_{1}^{2}+y_{3}^{2}\left(-y_{2}^{2}+y_{3}^{2}\right)}\right) T_{5}} \text { (C. } 54\right) \\
& U_{d}
\end{aligned}
$$




\section{Appendix D}

\section{Generators of $S O(6)$ and $S U(2)$}

Many calculations in the Bestest Little Higgs model depend on both the $6 \times 6$ generators corresponding to the $S U(2)$ subgroups of $S O(6)$, and the $S U(2)$ Pauli matrices. For completeness, these are included here.

The generators $T_{L}^{a}$ and $T_{R}^{a}$ are:

$$
\begin{gathered}
T_{L}^{1}=\frac{i}{2}\left(\begin{array}{cccccc}
0 & 0 & 0 & 1 & 0 & 0 \\
0 & 0 & 1 & 0 & 0 & 0 \\
0 & -1 & 0 & 0 & 0 & 0 \\
-1 & 0 & 0 & 0 & 0 & 0 \\
0 & 0 & 0 & 0 & 0 & 0 \\
0 & 0 & 0 & 0 & 0 & 0
\end{array}\right), T_{L}^{2}=\frac{i}{2}\left(\begin{array}{cccccc}
0 & 0 & 1 & 0 & 0 & 0 \\
0 & 0 & 0 & -1 & 0 & 0 \\
-1 & 0 & 0 & 0 & 0 & 0 \\
0 & 1 & 0 & 0 & 0 & 0 \\
0 & 0 & 0 & 0 & 0 & 0 \\
0 & 0 & 0 & 0 & 0 & 0
\end{array}\right), \\
T_{L}^{3}=\frac{i}{2}\left(\begin{array}{cccccc}
0 & 1 & 0 & 0 & 0 & 0 \\
-1 & 0 & 0 & 0 & 0 & 0 \\
0 & 0 & 0 & 1 & 0 & 0 \\
0 & 0 & -1 & 0 & 0 & 0 \\
0 & 0 & 0 & 0 & 0 & 0 \\
0 & 0 & 0 & 0 & 0 & 0
\end{array}\right), T_{R}^{1}=\frac{i}{2}\left(\begin{array}{cccccc}
0 & 0 & 0 & -1 & 0 & 0 \\
0 & 0 & 1 & 0 & 0 & 0 \\
0 & -1 & 0 & 0 & 0 & 0 \\
1 & 0 & 0 & 0 & 0 & 0 \\
0 & 0 & 0 & 0 & 0 & 0 \\
0 & 0 & 0 & 0 & 0 & 0
\end{array}\right),
\end{gathered}
$$




$$
T_{R}^{2}=\frac{i}{2}\left(\begin{array}{cccccc}
0 & 0 & -1 & 0 & 0 & 0 \\
0 & 0 & 0 & -1 & 0 & 0 \\
1 & 0 & 0 & 0 & 0 & 0 \\
0 & 1 & 0 & 0 & 0 & 0 \\
0 & 0 & 0 & 0 & 0 & 0 \\
0 & 0 & 0 & 0 & 0 & 0
\end{array}\right), T_{R}^{3}=\frac{i}{2}\left(\begin{array}{cccccc}
0 & -1 & 0 & 0 & 0 & 0 \\
1 & 0 & 0 & 0 & 0 & 0 \\
0 & 0 & 0 & 1 & 0 & 0 \\
0 & 0 & -1 & 0 & 0 & 0 \\
0 & 0 & 0 & 0 & 0 & 0 \\
0 & 0 & 0 & 0 & 0 & 0
\end{array}\right) .
$$

The Pauli matrices, $\tau^{a}$, are:

$$
\tau^{1}=\left(\begin{array}{ll}
0 & 1 \\
1 & 0
\end{array}\right), \tau^{2}=\left(\begin{array}{cc}
0 & -i \\
i & 0
\end{array}\right), \tau^{3}=\left(\begin{array}{cc}
1 & 0 \\
0 & -1
\end{array}\right)
$$

Also of note, the following $2 \times 6$ matrix, $M_{26}$ is necessary to contract the $S U(2)$ and $S O(6)$ matrices for the explicit symmetry breaking terms in the scalar potential. [3]

$$
M_{26}=\frac{1}{2}\left(\begin{array}{cccccc}
0 & 0 & 1 & i & 0 & 0 \\
1 & -i & 0 & 0 & 0 & 0
\end{array}\right)
$$

